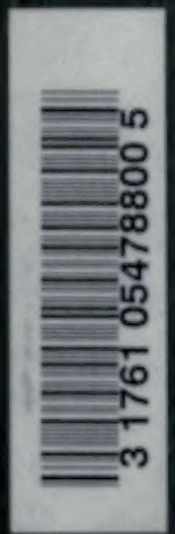


Firesentè

to the

BQ⿴囗十)

\{ุiniversity of đoronta

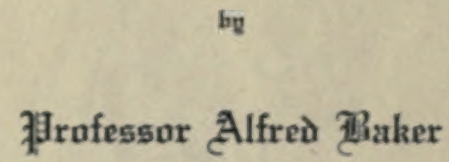

3)เาแe, 1940 


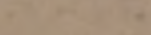

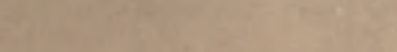

$$
\begin{aligned}
& =
\end{aligned}
$$

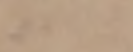
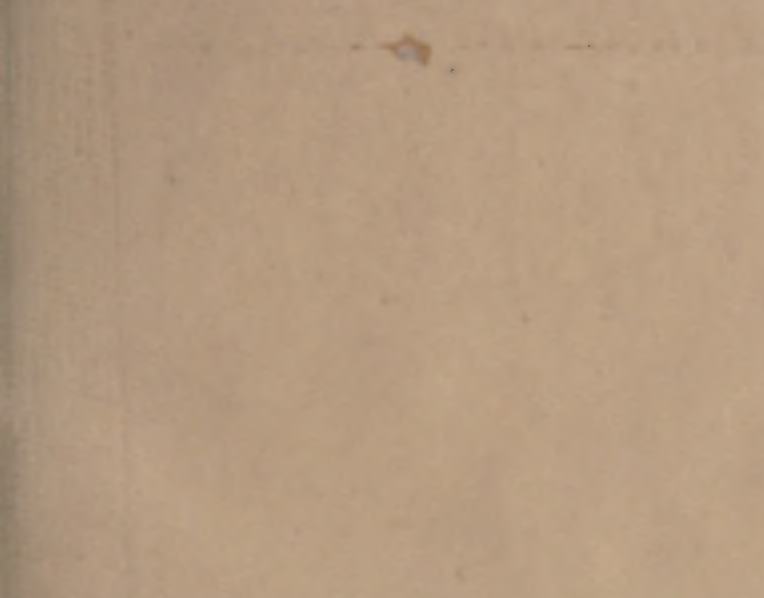
e 
ANALYTICAL STATICS. 


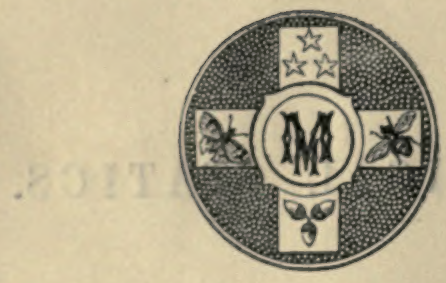


A Treatise ON

Yout ab 21:4

\section{ANAthticaL STATICS}

WITH NUMEROUS EXAMPLES.

By I. TODHUNTER, M.A., F.R.S.

EDIT:3d

THIND EDITTON, REVISHD AND ENLANGED.

1) Xoñon añ Cambrioge:

2) MACMILLAN AND CO.

3) 1866. 


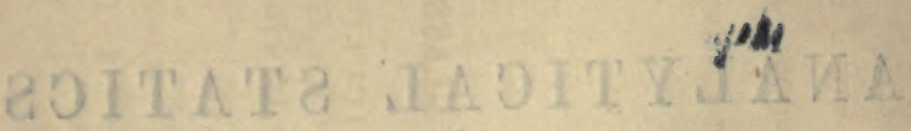

3:

Cambrioge:

PRINTED BY C. J. CLAY, M.A.

AT THE UNIVERSTTY PRESS.

IIBRARP
JUL 41967

ERSITY of TORO 


\section{PREFACE TO THE SECOND EDITION.}

Is this work will be found all the Propositions which usually appear in treatises on Theoretical Statics. To the different Chapters Examples are appended, which have been principally selected from the University and College Examination Papers; these will furnish ample exercise in the application of the principles of the subject.

Some of the Examples in the earlier Chapters asume results which are obtained at a later part of the book; the student who has no previous acquaintance with the subject may therefore, on his first perusal of the book, omit the more difficult Examples of the first six Chapters.

In the first three Chapters and in the ninth Chapter I have made considerable use of $\mathrm{Mr}$ Pratt's Treatise on Mechanical Philosophy, which was placed at my disposal by the Publishers.

In the second edition the work has been thoroughly revised and has received large additions; these additions have been made with the view of rendering the subject more readily intelligible by explaining and illustrating those parts which were found by the experience of teachers to be difficult for beginners.

\section{TODHUNTER.}

St Jois's Cohtror,

Aug. 28,1858 .

In the third edition many additions have been made, in order to illustrate the application of the principles of the subject to the solution of problems.

$$
\text { April, } 1866 .
$$




\section{Digitized by the Internet Archive in 2007 with funding from Microsoft Corporation}




\section{CONTENTS.}

Canar feca

I. Introduetsoa, Definitlases . . . . . . I

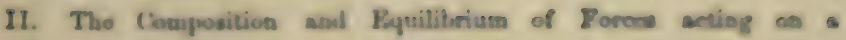

Partide . . . . . . . . \&

III. Renultant of Two Parallel Foreen Couples . . . o 80

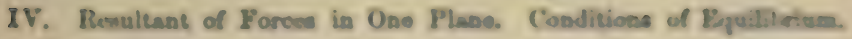

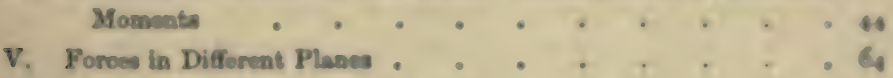

VI. Koquilibrium of a Constraiued Body . . . . . . Es

VII. Geaeral Theorems on a Siyoters of Ferces . . . . . . S

VIII. Centre of Gravity . . . . . . . . . 80

1X. Machines . . . . . . . 4 . 195

X. Friction . . . . . . . . 803

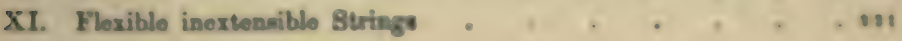

XII. Flexible extenaible Striags . . . . + . . as

XIII. Attractions . . . . . . . . . 86

XIV. Virtual Velocition . . . . . . . . 39s

Miscollaneoue Examples . . . . . . . . . 396 


\section{S T A T I C S.}

\section{CHAPTER I.}

INTRODLCTION.

1. A BODY is a portion of matter limited in erery dimetims, and is consequently of a determinate form and volume. A material particle is a boly indefinitely suall in every dirootion; wo shall speak of it for bloortsess as a particle.

2. A body is in motion when the body or its parts aceupy successivcly different positions in space. But we casthot judge of the state of rest or motion of a body withous coms. paring it with other bodics, and for this reason all motimens which come under our observation are necedsarily relaties motions.

3. Force is that which produces or tends to produec motion in a body.

4. When several forces act simultaneonsly on a body, is may happen that they neutralise each other; when a foly remains at rest though acted on by forces, it is said to be in equilibrium ; or, in other worls, the forces are said to maintain equilibrium.

5. Mechanics is the science which trents of the lawe of rest and motion of bodies. Staties treats of the lairs of the equilibrium of bodies, and Dymamies of the laws of motios of bodics.

6. There are three things to consider in a forme actis? on a particle: the position of the particle: the direstion of T. 8. 
the foree, that is, the direction in which it tends to make the particle start; and the intensity of the force. $\Lambda \mathrm{s}$ the dimensions of a particle are indefinitely small its position may be determined in the same manner as that of a point in goometry, and the direction of the force may be determined in the same manner as that of a straight line in geometry. We proceed then to consider the magnitude or intensity of a force.

7. Forces can be measured by taking some force as the unit, and expressing by numbers the ratios which other forees bear to this unit. Two forces are equal when being applied in opposite directions to a particle they maintain equilibrium. If we take two equal forces and apply them to a particle in the same direction we obtain a force double of either; if we unite three equal forces we obtain a triple force; and so on.

When we say then that a force applied to a particle is a certain multiple of another force, we mean that the first force may be supposed to be composed of a certain number of forces equal to the second and all acting in the same direction. In this way forces become measurable quantities, which can be expressed by numbers, like all other quantities, by referring them to a unit of their own kind. Forces may also be represented by straight lines proportional in length to these numbers, drawn from the point at which the forces act and in the directions in which they act.

8. Experience teaches us that if a body be let free from the hand, it will fall downwards in a certain direction; however frequently the experiment be made, the result is the: same, the body strikes the same spot on the ground in each trial, provided the place from which it is dropped remain the same. The canse of this undeviating effect is assumed to be an affinity which all bodies have for the carth, and is termed the force of attraction. If the body be prevented from falling by the interposition of a table or of the hand, the body exerts a pressure on the table or hand. Weight is the name given to the pressure which the attraction of the earth causes a body to exert on another with which it is in contact. 
9. A solid boly is conceived to be an serrepation of material particles which are beld together by their anumsel aftinities. This appears to be a bafe hypothesis, since experiruents shew that any body is divisible into sucoessirely mal.er and sunller portions without limit, if sufficient force be eserted to overcome the mutual action of the parts of the boly.

10. A rigid body is one in which the particles retain invariable positions with rewpect to each other. $\mathbf{S}_{0}$ body in nature is perfectly rigid; every body gields more or len to the forces which act on it. If, then, in any cuse this com. pressitility is of a sensible maignituile, we shall suppose that the boly lias assumed its figure of equilibrium, and thes consider the points of appliention of the forces an a system of invariable form. By body, liereafter, we mean rigial body.

11. When a force acts on a boly the effecs of the firce will be unchanged at whatever point of its dirvetion we nuspose it applied, provided this point be either one of vise prose of the body or be invariably connected with the loly. This principle is known by the name of the tramsmoisitility of a force to any point in its line of action; it is ansumed as an axiom or as an experimental fact. We may alow the amount of assumption involved in the axiom, by the fothew. ing process.

Suppose a body to be kept in equilibrium by a syatem of furces, one of which is the force $P$ applied at the point $A$. Thke any point $B$ which lies on the direction of this force, and suppose $B$ so connected with $A$ that the distance $A B$ is unchangeable. Then, if at 13 we introdnce two forcos, $P$ and $P^{\gamma}$, equal in macnitude and acting in opposite directims along the straight line $A B$, it socms evilent that no change is made in the eflect of the furce $P$ at $A$. Pet us now assume that $P$

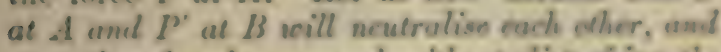
may therefore le remoed acithout disturining the equilitrium of the bealy; then there rrmains iloe furce $P$ at $I S$ producing the same effect os w i.en it acted at $A$.

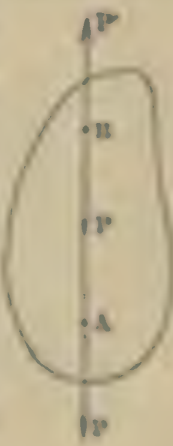

$1-2$ 
12. We shall have occasion hereafter to assume what may the called the commorae of the principle of the transmissibility of foroe, namely, that if a force can be transferred from its point of application to a second point without altering its effect, then the second point must be in the direction of the force. Nee Art, 17.

13. When we find it uscful to change the point of application of a force, we shall for shortness not always state that the new point is intariably connected with the old point, but this must be always understood. 


\section{$(5)$}

\section{CHAPTER II.}

\section{THE COMPOSITIOS ASD EQUHIMRILA OP POECTS ACTISG ON A PAKTICLE.}

14. Wmrs a particle is acted on by foreen which do not maintain equilibrium it will begin to move in sume determinate direction. It is clear then that a single furce may be found of sueh a magnituile, that if it acted in the dirsction gpposite to that in which the motion would take place th:s froe would prevent the motion, and consequently woull be in equilibrium with the other forces which het on the particle. If then we were to remove the original forces am replace them by a single force, equal in magnitude to that described above, but acting in the opponite direction, the particle would still remain at rest. This force, which is equara. lent in its effect to the combined effect of the original forces, is called their resultame, and the original forves are called the components of the resultant.

It will be necessary then to begin by declucing rules for the composition of forces; that is, for finding their resultant force. After we have determined these, it will be easy to deduce the analytical relations which forces must satisfy when in equilibrium.

15. To find the resultant of a given mumber of formes aceling mo a particle in the same straight line; and os find the condiction which they must satisfy that they way be in egwolobrium.

When two or more forces act on a particle in the mame direction it is evident that the resultant furce is erual to their sum and acts in the same direction.

When two forces act in different directions, but in the same straight line, on a particle, it is equally clear that their rosultant is equal to their differenoc and acts in the direction of the greater component. 
When several forees act in different directions, but in the same straight line, on a particle, the resultant of the forces scting in one direction is equal to the sum of these forces, and acts in the same direction; and so of the forces acting in the opposite direction. The resultant, therefore, of all the furces is equal to the difference of these sums, and acts in the direction of the greater sum.

If the forces acting in one direction are reckoned positive, and those in the opposite direction negative, then their resultant is equal to their algebraical sum; its sign determines the direction in which it acts.

In order that the forces may be in equilibrium, their resultant, and therefore their algebraical sum, must vanish.

16. There is another case in which we can easily determine the magnitude and direction of the resultant.

Iet $A B, A C, A D$ be the directions of three equal forces acting on the particle $A$; suppose these forces all in the same plane and the three angles $B A C, C A D, D A B$ each equal to $120^{\circ}$; the particle will remain at rest, for there is no reason why it should move in one direction rather than another. Each of the forces is therefore equal and opposite to the resultant of the other two. But if we take on the directions of two of them, $A B, A C$, two equal straight lines $A G, A H$ to represent the forces, and complete the parallelogram GAHE, the diagonal $A E$ will lie in the same straight line with $A D$. Also the triangle $\triangle G E$ will be equilateral, and therefore $A E=\Lambda G$. Hence, the diagonal $A E$ of the parallelogram constructed on $A G, A H$ represents

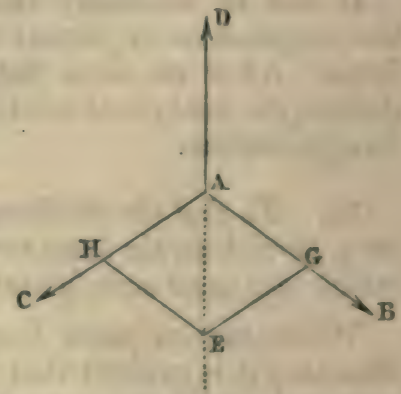
the resultant of the two forces which $A G$ and $A I I$ respectively represent.

\section{This proposition is a particular case of one to which we now proceed.}


17. If toro furces acling at a poine be mpresenend in diras. tiun and mangieule by ties seraight lines drates fres the point, and a parallelogram be described on these atratight lines as adjacent sides, then the resultant will be representeal in ditrection and magnitude by chat diagomal of the paralledeyram which passes through the point.

This Proposition is called the Rurulleloyran of firces.

\section{To find the direction of the renultant.}

When the forces are equal it is clens that the direction of the resulcant will bimat the angle between the directima of the forces; or, if we represent the furees in mapnimis and direction by two ntraight lines drawn from the point where they aet, and deseribe in parallelogram on theser atraighe lines, that diagonal of the parallelognam which paseces through the point will be the direction of the resultant.

Let us assume that this is true for forces $p$ and $m$ inclincd at any angle, and also for forces $p$ and $n$ inefined at the same angle; we can shew that it must then be true for two furves $p$ and $m+n$ also inclined at the same angle.

Iet $A$ be the point at which the forces $p$ and $m$ aes; $A B, A C$ their directiona and proportional to them in magnitude: complete the parallelogram $B C$, and draw the diagonal $A D$; then, by hypothesis, the resultant of $p$ and $m$ acts along $A D$.

$A$ gain, take $C E$ in the anme ratio to $A C$ that $n$ bears to m. By Art. 11

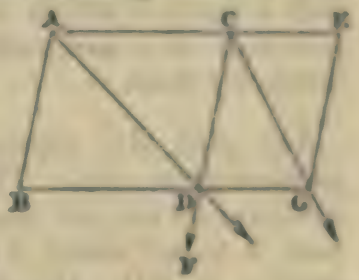
we may suppose the force $n$ which acts in the direction $A B$ to he applied at $A$ or $C$; and therefure the forces $p, m$, and $w$. in the straight lines $A B, A C$, and $C E$, are the arme $a p$ and $w+n$ in the straight lines $A B$ and $A E$.

Now replace $p$ and $n$ by their resultant and tranoine ita point of application from $A$ to $D$; then resoles this form at $D$ into two parallel to $A B$ and $A C$ regpoctively: them resolved parts nust evidently be $p$ and $\omega$, the former acting in the diruction $D F$, and the latter in the dirvetion $D Q$. Then transfer $p$ to $C$ and $m$ to $C$. 
But, by the hypothesis, $p$ and $n$ acting at $C$ have a resultant in the direction $C G$; therefore $p$ and $n$ may be replaced by their resultant and its point of application transferred to $G$. And $m$ has also been transferred to $G$. Hence by this process we have removed the forces which acted at $A$ to the point $G$ without altering their effect. We may infer then (see Art. 12) that $G$ is a point in the direction of the resultant of $p$ and $m+n$ at $A$; that is, the resultant of $p$ and $m+n$ acts in the direction of the diagonal $A G$, provided the hypothesis is correct. But the hypothesis is correct for equal forces, as $p, p$, and therefore it is true for forces $p, 2 p$; consequently for $p, 3 p$, and so on; hence it is true for $p, r \cdot p$.

Hence it is true for $p, r \cdot p$, and $p, r \cdot p$, and consequently for $2 p, r \cdot p$, and $s 0$ on; and it is finally true for $8 . p$ and $r . p$, where $r$ and $s$ are positive integers.

We have still to shew that the Proposition is true for incommensurable forces.

This may be inferred from the fact that when two magnitudes are incommensurable, so that the ratio of one to the other cannot be expressed exactly by a fraction, we can still find a fraction which differs from the true ratio by a fraction less than any assigned fraction. Or it may be established indirectly thus.

Iat $A B, A C$ represent two such forces. Complete the parallelogram $B C$. Then if their resuliant do not act along $A D$ suppose it to act along $A E$; draw $L F$ parallel to $B D$. Divide $A C$ into a number of equal portions, each less than $D E$; mark off from $C D$ portions equal to these, and let $K$ be the lnst division; this evidently

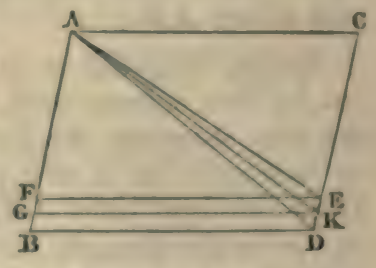
falls between $D$ and $E$; draw $G K$ parallel to $A C$. Then two forces represented by $A C, A G$ have a resultant in the direction $A K$, because they are commensurable; therefore the forces $A C$ and $A B$ are equivalent to $A K$ together with a force equal to $G B$ applied at $A$ along $A B$. And we may assume as obvious that the resultant of these forces must lie 
beteeen $A K$ and $A B$; but by supponition the resuleant is $A E^{\circ}$ which is not between $A K$ and $A B$. This is almurd.

In the same manner we may shew that every dimetisa besides $A D$ leads to an absurdity, and therrfore the mosteant must act along $A D$, whether the forces be commeturusalte op incommensurable.

II. To find the magnitude of the rorultant.

Iet $A B, A C$ be the directions of the given foreen, $A D$ that of their resultant; take $A E$ opposite to $A D$, and of such a length as to represent the magnitule of the reanltant. Then the forcrs represented by $A B, A C, A E$, balance each other. On $A E$ and $A B$ as adjacent vides construet the parallelogram $A B F E$; then the diagonal $A F$ is the direction of the resultant of $A E$ and $A B$.

Hence $A O$ is in the same straight line with $A F$; hence $F D$ is a parallelogram; and therefore $A E=F B=A I$. Hence the $\pi$ sultant is represented in magnitule ss well

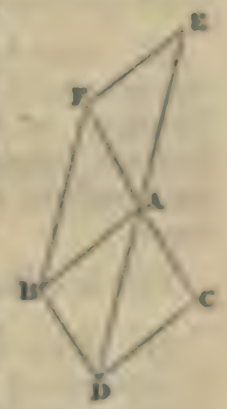
as in direction by the diagonal of the parallelogram.

Thus the proposition called the Purallelogram of Forces is complefely established.

18. Hence if $P$ and $Q$ repreaent two component forces acting at an angle $a$ on a particle, the resultant $\mathbb{R}$ is gircu by the equation

$$
R^{n}=P^{e}+Q^{P}+2 P Q \cos \alpha .
$$

19. When three forces acting on a particle are in murlitirium they are respectivaly in the same propartion as the sines of the angles included by the dirations of the ender 8000.

For if we refer to the thind figure of Art. 17 we have

$$
\begin{aligned}
P: Q: R & : A B: A C(\text { or } B D): A D \\
& \because \sin A D B: \sin B A D: \sin A B D \\
& \because \sin C A E: \sin B A E: \sin B A C .
\end{aligned}
$$


Conversely if three forces act on a particle, and each force is as the sine of the angle between the directions of the other iwo, it may be shewn that one of the forces is equal in magvitude to the resultant of the other two, and acts either in the same direction or in the opposite direction: in the latter case the three forces are in equilibrium.

It should be noticed that if the sides of a triangle be drawn parallel to the directions of the forces, the length of any side will be proportional to the sine of the angle between the forces which correspond to the other two sides.

20. Any force acting on a particle may be replaced by two others, if the sides of a triangle drawn parallel to the directions of the forces have the same relative proportion that the forces have. For by the parallelogram of forces the resultant of the latter two forces is equal to the given force.

This is called the resolution of a force.

21. Since the resultant of two forces acting on a particle is represented in magnitude and direction by the diagonal of the parallelogram constructed upon the straight lines which represent these forces in magnitude and direction, it follows that, in order to obtain the resultant of the forces $P_{1}, P_{2}, P_{9}, \ldots$ which act on a particle $A$, and are represented by the straight lines $A P_{1}, A P_{2}, A P_{s}, \ldots$ we may proceed as follows.

Find the resultant of $P_{1}$ and $P_{3}$, compound this resultant with $P_{s}$, this new resultant with ${ }^{2}$, and so on. It follows

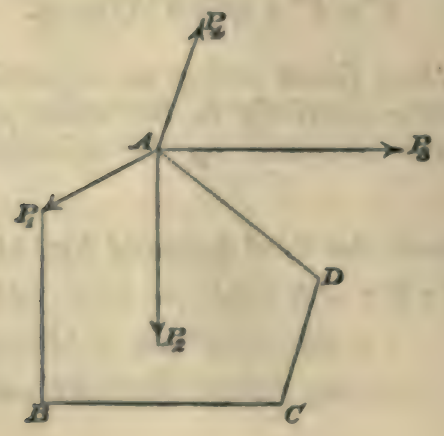


from this, that if we construct a polygon $A P_{1} B C D$, of whith the sides are respectively equal and parallel to the sinaige lines $A P_{1}, A P_{v} \ldots$ and foin $A$ with the last verter $D$, the straight line $A D$ will reprement in magnitude asd discetion the resultant of all the forces.

We may conclude that the necesary and mufficient condition for the equilibrium of a number of forces arsing oo a particle is, that the point $D$ should coincide with $A$ : that is, that the figure $A P, B \ldots D$ should be a cumpice polygon. The forces in the figure are not necesenrily alt in one plane.

The rosult here obtained may be enunciated thus: If the nieles of any polygon taken in order are reopecticely propertional to the magnitudes of forcos acting at a point, and parcillet to the directions of the forces, then the forces scill ke in cgunibirium.

This proposition is called the Polygon of Funces.

The student must carefully notice the conditions under which this proposition is asserted to hold; the forces ane supposed all to act at one point, and are to be represented by tas sides of a polygon taken in order. As an example of the latter condition, suppose a quadrilateral $A B C D$; then if forcon which may be represented by $A B, B C, C D, D A$, act at a point the forces will be in equilibrium: but the forces will not he in equilibrium if represented by $A B, B C, D C, D A$, or by $A B, B C, C D, A D$.

The direction and magnitude of the resultant may also be determined analytically, as in the following Articles.

22. Any number of furces act on a particle in ane plane: required to find the magnitude and direction of their resmiband.

Iet $P_{v}, P_{v}, P_{2} \ldots$ bo the forces, and $a_{1}, a_{1}, a_{0} \ldots$ the anglea their directions make with a fixed atraight line drawn tironght the proposed point. Take this fised straight line for the asxis of $x$, and one perpendicular to it for that of $y$. Thien, by Art, $20, P_{3}$ may be resolved into $P_{3}$ cos $\alpha_{1}$, and $P_{3}$, sin a, acting along the ases of $x$ and $y$ respectively. The other furcess may 
be similarly resolred. By algebraical addition of the forces which act in the same straight line we have

$P_{1} \cos \alpha_{1}+P_{2} \cos \alpha_{3}+P_{3} \cos \alpha_{3}+\ldots$ along the axis of $x$,

$P_{1} \sin \alpha_{1}+P_{2} \sin \alpha_{3}+P_{3} \sin \alpha_{3}+\ldots$ along the axis of $y$.

We shall express the former by $\Sigma P \cos \alpha$ and the latter by $\Sigma P \sin \alpha$, where the symbol $\Sigma$ denotes that we take the sum of all the quantities of which the quantity before which it is placed is the type.

If we put $P_{1} \cos \alpha_{1}=X_{1}$ and $P_{1} \sin \alpha_{1}=Y_{1}$, and use a similar notation for the other components, we have two forces replacing the whole system, namely $\Sigma X$ along the axis of $x$ and $\Sigma Y$ along that of $Y$. If $R$ denote the resultant of these forces and $a$ the angle at which it is inclined to the axis of $x$, we have, by Art. 17,

Also

$$
\begin{gathered}
R^{2}=(\Sigma X)^{2}+(\Sigma Y)^{2}, \\
\tan a=\frac{\Sigma Y}{\Sigma X^{*}} \\
\cos a=\frac{\Sigma X}{R} ; \quad \sin a=\frac{\Sigma Y}{R} .
\end{gathered}
$$

23. To find the conditions of equilibrium when any number of forces act on a particle in one plane.

When the forces are in equilibrium we must have $R=0$; therefore

$$
(\Sigma X)^{2}+(\Sigma Y)^{2}=0
$$

therefore

$$
\Sigma X=0 ; \quad \Sigma Y=0 \text {; }
$$

and these are the conditions among the forces that they may be in equilibrium.

24. Three forces act on a particle in directions making right angles with each other; required to find the magnitude and direction of their resultant.

Let $A B, A C, A D$ represent the three forces $X, Y, Z$ in magnitude and direction. Complete the parallelogram $B C$, 
and draw the dingonal $A E$; then $A E$ mprenents the prenitang of $X$ and $Y$ in magnitude assd dircetion, by Are 18. Xive

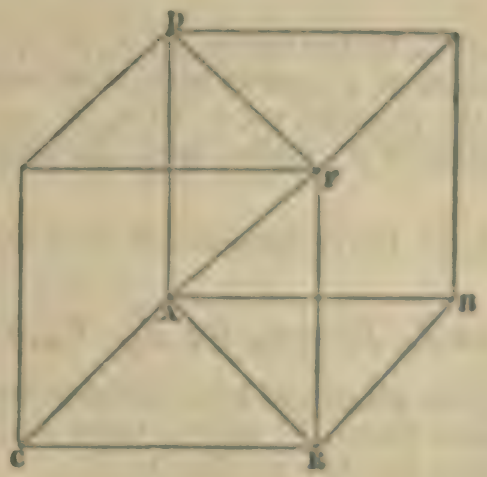

the resultant of this force and $Z$, that is of the forces mpresented by $A E, A D$, is represented is magnitude and dimction by $A F$, the diagonal of the parallelogram $D E$. Hence the resultant of $X, Y, Z$ is represented in magnituite and direction by $A F$. Let $R$ be the magnitude of the resultans, and a, $A, C$ the angles the direction of $R$ makes with those of $\bar{x}, \bar{Y}, \mathcal{Z}$ Then, since

$$
A F^{n}=A E^{n}+A D^{0}=A B^{0}+A C^{n}+A D^{2},
$$

therefore

$$
I^{n}=X^{2}+I^{2}+X
$$

$$
\Lambda \text { lso } \cos a=\frac{A B}{A F}=\frac{X}{I}, \cos b=\frac{A C}{A F}=\frac{Y}{R}, \cos c=\frac{A D}{A F}=\frac{Z}{L^{6}} .
$$

Thus the magnitude and direction of the resaltant ane decer. mined.

25. It follows from the last Article that any fone $I i$ the direction of which makes the angles $a, b$, with tilres mosangular axes fixed in spaco, may be rplaced by the stime forres $R \cos a, R \cos b, R$ cus 6 . aleting simultaneosaly on the particle on which $R$ acts, and having their diructiusas parallad to the axes of coordinates respectirity. 
26. Any number of forces act on a particle in any directions; required to find the magnitude and direction of their resultant.

Let $P_{1}, P_{n}, P_{3}, \ldots$ be the forces; let $\alpha_{1}, \beta_{1}, \gamma_{1}$ be the angles which the direction of $P_{2}$ makes with three rectangular axes drawn through the proposed point; let $\alpha_{2}, \beta_{8}, \gamma_{8}$ be the angles which the direction of $P_{s}$ makes with the same axes; and 80 on.

Then, by Art. 25, the components of $P_{1}$ in the directions of the axes are

$$
P_{1} \cos \alpha_{1}, P_{1} \cos \beta_{1}, P_{1} \cos \gamma_{1} \text {, (or } X_{1}, Y_{1}, Z_{1} \text {, suppose). }
$$

Resolve each of the other forces in the same way, and reduce the system to three forces, by adding those which act in the same straight line, Art. 15; we thus have

$$
\begin{aligned}
& P_{1} \cos \alpha_{1}+P_{2} \cos \alpha_{2}+\ldots \text { or } \Sigma P \cos \alpha_{1} \text { or } \Sigma X, \\
& P_{1} \cos \beta_{1}+P_{2} \cos \beta_{2}+\ldots \text { or } \Sigma P \cos \beta, \text { or } \Sigma Y, \\
& P_{1} \cos \gamma_{1}+P_{2} \cos \gamma_{2}+\ldots \text { or } \Sigma P \cos \gamma, \text { or } \Sigma Z,
\end{aligned}
$$

acting in the directions of the axes of $x, y$, and $z$ respectively.

If we call the resultant $R$, and the angles which its direction makes with the axes $a, b, c$, we have, by Art. 24,

$$
R^{2}=(\Sigma X)^{2}+(\Sigma Y)^{2}+(\Sigma Z)^{2} \text {, }
$$

and $\quad \cos a=\frac{\Sigma X}{K}, \cos b=\frac{\Sigma Y}{K}, \cos c=\frac{\Sigma Z}{K}$.

27. To find the conditions of equilibrium when any number of forces act on a particle.

When the forces are in equilibrium, we must have $R=0$; therefore

$$
(\Sigma X)^{2}+(\Sigma Y)^{2}+(\Sigma Z)^{2}=0,
$$

therefore

$$
\Sigma X=0 ; \Sigma Y=0 ; \Sigma Z=0 \text {; }
$$

and thear are the conditions among the forces that they may be in equilibrium. 
28. The expression for the inngnitude of the resulems in Art. 26 mag be rendered independems of the poaituon of the axcs. For, from Art. 26,

$$
\begin{aligned}
& K^{N}=\left(P_{1} \cos \alpha_{1}+P_{0} \cos \alpha_{0}+\ldots\right)^{0}+\left(P_{1} \cos \beta_{1}+P_{0} \cos \beta_{1}+\ldots\right)^{0} \\
& +\left(P_{1} \cos \gamma_{1}+P_{\mathrm{t}} \cos \gamma_{\mathrm{t}}+\ldots\right)^{\mathrm{P}} \text {. }
\end{aligned}
$$

When the expressions on the right-hand side ane developed, we shall find that the cocflicient of $P_{i}^{\prime}$ is

$$
\cos ^{2} \alpha_{1}+\cos ^{\circ} \beta_{1}+\cos ^{0} \gamma_{1} \text {, }
$$

and that the cocficient of $P_{\mathrm{r}} P_{\mathrm{v}}$ is

$$
2\left(\cos \alpha_{1} \cos \alpha_{0}+\cos \beta_{1} \cos \beta_{0}+\cos \gamma_{1} \cos \gamma_{0}\right) \text {. }
$$

Now we know from Analytical Geometry of three dimeraiom that

\section{and that}

$$
\cos ^{\mathrm{N}} \alpha_{1}+\cos ^{\mathrm{A}} \beta_{1}+\cos ^{\mathrm{N}} \gamma_{1}=1 \text {; }
$$

$$
\cos \alpha_{1} \cos \alpha_{0}+\cos \beta_{2} \cos \beta_{0}+\cos \gamma_{1} \cos \gamma_{0}
$$

is equal to the cosine of the angle between the directions of the forces $P_{1}$ and $P_{2}$, which we may dehote by con $\left[P_{n}, P J\right.$. Similar values will be found for the cocticients of the vthis terms; and the result may be expressed thas,

$$
R^{\circ}=\Sigma P^{\circ}+2 \mathbb{\Sigma} P P^{\prime} \cos \left(P, P^{\prime}\right)
$$

where by $P, P^{\prime}$ we mean any two of the forces.

29. The equation $R \cos a=\Sigma P$ ens $\alpha$, in Ar. 26, shewr that the resoleod part of the resultume in any dirmbiom is comal to the sum of the resolved purts of the ceimponests in the ninme dircetion; for since the axes were taken arutranily, that of $\mathrm{z}$ might have been male to coincide with any nongreal dirro-

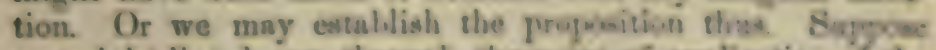
a straight line drawn through the jrunt of apptication of the forces, and inclined to the axes at angles $\alpha, \beta ; \gamma$. Take the three equations of Art. 26,

$$
\begin{aligned}
& R \cos a=P_{1} \cos q_{1}+P_{1} \cos \alpha_{0}+\ldots \ldots \\
& R \cos b=P_{1} \cos \beta_{1}+P_{1} \cos \beta_{1}+\ldots \ldots \\
& R \cos \mathrm{c}=\Gamma_{1} \cos \gamma_{1}+\Gamma_{\mathrm{c}} \cos \gamma_{\mathrm{t}}+\ldots \ldots
\end{aligned}
$$


Multiply the first by $\cos \alpha^{\prime}$, the second by $\cos \beta$, and the third by $\cos \gamma$, and add. Then, if $\theta_{1}, \theta_{2}, \ldots$ denote the angles which $P_{1}, P_{2} \ldots$ make with the arbitrarily drawn straight line, and $\theta$ the angle which the resultant $R$ makes with it, we have, by the formula quoted in Art. 28 for the cosine of the angle between two straight lines

$$
R \cos \theta=P_{1} \cos \theta_{2}+P_{2} \cos \theta_{2}+\ldots \ldots
$$

30. From Art. 20 it is obvious that a given force may be resolved into two others in an infiuite number of ways. When we speak of the resolved part of a force in a given direction, as in the preceding $\Lambda$ rticle, we shall always suppose, unless the contrary is expressed, that the given force is resolved into two forces, one in the given direction and the other in a direction at right angles to the given direction. The former component we shall call the resolved force in the given direction.

When forces act on a particle it will be in equilibrium, provided the sums of the forces resolved along any three directions not lying in one plane are zero. For if the forces do not balance, they must have a single resultant; and as a straight line cannot be at right angles to three straight lines which meet at a point and are not in the same plane, the resolved part of the resultant, and therefore the sum of the resolved parts of the given forces, along these three straight lines, could not vanish, which is contrary to the hypothesis.

31. In $\Lambda$ rt. 26 we resolved each force of a system into three others along three rectangular axes. In the same way we may, if we please, resolve each force along three straight lines forming a system of oblique axes. For whether the figure in Art. 24 represent an oblique or rectangular parallelepiped, the force $A F$ may be resolred into $A D$ and $A E$, and the latter again resolved into $A B$ and $A C$. Hence the resultant of a system of forces may be represented by the diagonal of an oblique parallelepiped, and for equilibrium it will be necessary that this diagonal should vanish, and therefore that the edges of the parallelepiped should vanish.

The following three articles are particular cases of the equilibrium of a particle. 
82. To desermine the condition of equilitirium of a pareite acteal on by any forces and constrained to remasin on a gives somerech curve.

By a moeeth curve we understand a curve thas can esly excif force on the partiele in a direction mormal to the cuere at the print of contact.

Iet $\boldsymbol{X}, \boldsymbol{Y}, \boldsymbol{Z}$ denote the froces acting on the particle is directions parallel to three rectangulas axe, exritusire of the action of the curve. Let $x, y, z$ denote the eo-ondinates of the particle, and s the length of the are measured from soene fixed point up to the point $(x, y, s)$. Then by Aralyiol Geometry of three dimensions the cosines of the anglos whish the tangent to the curve at the print $\{x, y, 2\}$ makos with the axes are $\frac{d x}{d_{s}}, \frac{d y}{d a}, \frac{d s}{d a}$, reapectively. The forcen acting en the particle being rewolved along the tangent to the ensre. their sum is

$$
x \frac{d s}{d s}+Y \frac{d y}{d s}+Z \frac{d s}{d s} .
$$

Unless this vanishes, there will be nothing to prevent the particle from moring; for equilibrium then we mast have

$$
X \frac{d x}{d s}+Y \frac{d y}{d s}+Z_{d s}^{d s}=0 .
$$

Conrersely if this relation holds the particle will mosain s: rest, for there is no furce to make it move along the cors. which is the only motion of which it is capable.

We have supposed the particle to be placed insile a $\mathrm{mos}^{\mathrm{t}} \mathrm{r}$ which has the form of the curve. 18, however, the punthle fe merely placed in contact orith a corres it will le furhier sines: sary for equilibrium shat the resultant of the forres sheath press the particle against the curve and avt move is $f \cdot m$ the curve.

33. To determine the cunditions of eqsili rien of a pursid acted on by any forces and constrainal to rumeis as a gina smeoth surficec.

T. $\mathrm{s.}$ 
A smooth surface is one which can exert no force on the particle except in a direction normal to the surface.

Let $X, Y, Z$ denote the forces acting on the particle in directions parallel to three rectangular axes, exclusive of the action of the surface. 'The resultant of $X, Y, Z$ must act in a direction normal to the surface at the point where the particle is situated; for if it did not, we might decompose it into two forces, one in the normal and one at right angles to the normal, of which the latter would set the particle in motion. The cosines of the angles which the resultant of $X, Y, Z$ makes with the axes are proportional to $X, Y, Z$ respectively; and if $F(x, y, z)=0$ be the equation to the surface, the cosines of the angles which the normal to the surface at the point $(x, y, z)$ makes with the axes, are by Analytical Geometry of three dimensions proportional to $\frac{d F}{d x}, \frac{d F}{d y}$, and $\frac{d F}{d z}$ respectively. Hence for equilibrium we must have

$$
\frac{X}{\frac{d F}{d x}}=\frac{Y}{\frac{d F}{d y}}=\frac{Z}{\frac{d F}{d z}} .
$$

If these relations are satisfied, the resultant force is directed along the normal; hence, if we suppose the particle incapable of leaving the surface, the above conditions will be sufficient to ensure its equilibrium; but if the particle be merely placed on a surface, it will be further necessary that $X, Y, Z$ should act so that their resultant may press the particle against the surfuce. For example, if the particle be placed on the outside of a sphere, the resultant of $X, Y$, and $Z$ must act towards the centre of the sphere.

34. Suppose it required to determine the action which the curve or the surface exerts on the particle in the preceding cases. Denote it by $R$, and let $\alpha, \beta, \gamma$ be the angles its direction makes with the axes. Since $I ?$ and the forces $X, Y, Z$ maintain the particle in equilibrium, we have by $A$ rt. 27 ,

$$
R \cos \alpha+X=0, R \cos \beta+Y=0, R \cos \gamma+Z=0 \ldots . .(1) \text {. }
$$


Also when the particle resta on a curve surface whose equatis is $F(x, y, z)=0$, $\cos x$, cos $\beta$, anil con $\gamma$ are known is trums of the co-ordinates of the purviele, aibere they are proporninal to $\frac{d F}{d x}, \frac{d F}{d y}, \frac{d F}{d y}$ respectively. Hence the equationa (1) and that to the surface will determine $x, y, z$, and $R$, if $x, I, Z$ be given.

If the particle rest on a curve line, then, since the direction of $R$ is propendicular to that of the tangrat to the carve, we have the following equation from Asalficeal Goonsersy of three dimensions,

$$
\cos 2 \frac{d x}{d s}+\cos \theta \frac{d y}{d s}+\cos \gamma \frac{d z}{d s}=0
$$

Since $\frac{d x}{d s}, \frac{d y}{d s}$, and $\frac{d s}{d s}$ can he expresect, thenestially at lenst, in terms of $x, y$, and $s$, the equation (2) gives s mlatien between $\cos \alpha, \cos \beta$, and $\cos \gamma$, and $x, y$, and $z$. Thes (1) ased (2) wigether with the two equations to the curve and the equation

$$
\cos ^{2} \alpha+\cos ^{2} \beta+\cos ^{2} \gamma=1
$$

are sufficient to determine the seven qquantities $I_{0}, s_{5}, y_{1} s$, $\cos \alpha, \cos \beta$, and $\cos \gamma$.

We may observe that from (1)

$$
\boldsymbol{E}^{3}=\boldsymbol{X}^{3}+\boldsymbol{r}+\boldsymbol{Z} \text {. }
$$

35. Duchayla's proof of the Parallelogram of Foroes wlich we have given in Art. 17, rests on the principle of tise sreas: missibility of foree; see Art. 11. Wo shall grve amestra joof which does not intolve this principle; this jroot is Potson's with a slight modification. We nssume that if two gyal forces act on a particle, the direction of the resultant biscets the angle hetween the directions of the components. Alim, if $P$ 'denote the magnitude of each of two equal foroes, $2 x$ the angle between their dirvetions, and $I$ the magnitue in ef the resultant, then $l i$ must bo some function of $P$ aind ab swipen

$$
n=f(P, \infty) \text {. }
$$

In this equation, if we change our unit of force, the numerical 
values of $P$ and $R$ will change; but as the above equation must be true, whatever unit of force we adopt, it follows that the function $f(P, x)$ must be of the form $P \phi(x)$. Hence we have

$$
R=P \phi(x) \text {. }
$$

Let $M$ represent the position of the particle; $M A, M I$ ? the directions of the equal forces acting on it; $M D$ the direction of the resultant. Draw the four straight lines $M C, M G, M I I, M E$, making the angles $C M A, G M A, H M B, E M B$ all equal, and let $z$ denote the magnitude of each angle. Suppose the force $P$ acting along $1 L A$ to be resolved into two equal forces acting along $M C$ and $M G$ respectively; denote each of these components by $Q$; then

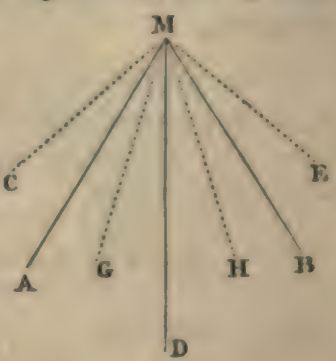

$$
P=Q \phi(z) \text {. }
$$

Resolve $P$ acting along $M B$ in like manner into two forees earl, equal to $Q$, acting along $M E$ and $M H$ respectively. Thus the two forcss $P$ are replaced by the four torces $Q$; and consequently the resultant of these four forces must coincide in magnitude and direction with the resultant $I R$ of the two forces $P$.

I.et $Q^{\prime}$ denote the resultant of the two forces $Q$, acting along $M G$ and $M H$; since $G M D=I I M I D=x-z$, we have

and $M D$ is the direction of $Q^{\prime}$.

$$
Q^{\prime}=Q \phi(x-\varepsilon),
$$

Similarly, the resultant $Q^{\prime \prime}$ of the other forces $Q$ will act along $M D$; and since $C M D=E M D=x+z$, we have

$$
Q^{\prime \prime}=Q \phi(x+z) \text {. }
$$

Since $Q^{\prime}$ and $Q^{\prime \prime}$ both act along the straight line $M D$, their resultant, which is also the resultant of the four forces $Q$, must be equal to their sum; hence

$$
R=Q^{\prime}+Q^{\prime \prime} \text {. }
$$

But we have $\left.\quad R=P \phi(x)=Q \phi(z) \phi^{\prime} x\right)$.

Iler.ce $\phi(x) \phi(z)=\phi(x+z)+\phi(x-z)$ 
This equation almits of more than one solation : fir exsens. ple, if $\phi(x)=2 \cos c x$, or if $\phi(x)=e^{2}+c^{\circ}$, where $e$ in asy cunstant, the equation is aatisfied; we shall lewwere slew shat the only solution adraissible in the present gुaention is t'is following,

$$
\phi(x)=2 \cos x \ldots \ldots \ldots \ldots \ldots \ldots \ldots \ldots \ldots \ldots \ldots \text { (2) }
$$

We may observe that we need not consider any valae of a greater than $\frac{\pi}{2}$, for the directions of two forcen acting at a point will always ineluile an angle leas than $\pi$ : we may thea assume it as obvivous that $\phi(x)$ usust be a positire quasitity.

We shall first shew that if $\phi(x)=2 \cos x$ when $a$ has any value $a$, then $\phi(x)$ must $=2 \cos x$ when $x$ has the value $\frac{a}{2}$. In (1) put $x$ and 2 each equal to $\frac{a}{2}$, so that $\phi(x+s)$ lecomes equal to $2 \cos a$; thus

$$
\phi\left(\frac{a}{2}\right) \phi\left(\frac{2}{2}\right)=\phi\left(0^{\circ}\right)+2 \cos 2
$$

But the resultant of two equal forces acting in the same straight line is equal to twice either of the component firces; thus $\phi(0)=2$; therefure by (3)

$$
\phi\left(\frac{a}{2}\right) \phi\left(\frac{a}{2}\right)=2(1+\cos \alpha)=4 \cos ^{2} \frac{a}{2} \text {. }
$$

Hence $\phi\left(\begin{array}{l}a \\ 2\end{array}\right)= \pm 2 \cos \frac{a}{2} ;$ but by supprosition $\frac{a}{2}$ is less thas $\frac{\pi}{2}$, and $\phi\left(\frac{a}{2}\right)$ must be a pasibice qृaantity; thus

$$
\phi\left(\frac{a}{2}\right)=2 \cos \frac{\alpha}{2} \text {. }
$$

Similarly if $\phi(x)=2 \cos x$ when $x=\frac{a}{2}$, then $\phi[x]=2 \cos x$ when $x=\frac{\alpha}{4}$; and so on. Thus we conclule that if $\phi(x)=2 e=x$ 
when $x=\alpha$, then $\phi(x)=2 \cos x$ when $x=\frac{\alpha}{2^{*}}$, where $x$ is any positive integer.

We shall next shew that if $\phi(x)=2 \cos x$ when $x=\beta$, and when $x=\gamma$, and when $x=\beta-\gamma$, then $\phi(x)=2 \cos x$ when $x=\beta+\gamma$. From (1)

$$
\begin{aligned}
\phi(\beta+\gamma) & =\phi(\beta) \phi(\gamma)-\phi(\beta-\gamma) \\
& =4 \cos \beta \cos \gamma-2 \cos (\beta-\gamma) \\
& =2 \cos (\beta+\gamma) .
\end{aligned}
$$

Thus if (2) holds when $x=\beta$, it will hold when $x=2 \beta$; this we obtain by supposing $\gamma=\beta$. Then if (2) holds when $x=\beta$ and when $x=2 \beta$, it also holds when $x=3 \beta$; and so on; that is, if (2) holds when $x=\beta$ it will hold when $x=m \beta$. Thus we conclude that if (2) holds when $x=\alpha$ it will hold when $x=\frac{m a}{2^{m}}$, where $m$ and $n$ are any integers.

But since the numbers $m$ and $n$ may be as great as we please, we can take them such that the expression $\frac{m \alpha}{2^{n}}$ may differ as little as we please from any assigned value of $x$. We may therefore consider (2) as completely demonstrated if it holds for any value of $x$ different from zero. But by Art. 16, it does hold when $x=\frac{1}{3} \pi$, for then $\phi(x)=1=2 \cos \frac{1}{3} \pi$; hence it holds always. Hence

$$
R=2 P \cos x \text {. }
$$

If then the forces $P$ be represented by straight lines drawn from their point of application, the resultant $R$ will be represented by that diagonal of the parallelogram described on these straight lines which passes through the point of application.

Next, let two unequal forces $P$ and $Q$ act on the particle $M$ along the straight lines $M A$ and $M B$; represent their intensitics by the straight lines $M G$ and $M / I$ taken on their directions, and complete the parallelogram $M G K M$.

First suppose $A M B$ a right angle. Draw the two diagonals $M K$

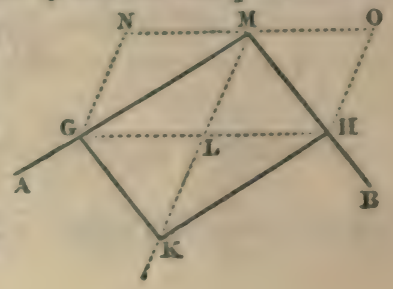


and $G I I$, which meet at $L$; throngh $G$ and $I I$ drtw $O . S$ and $I I O$ probllet to $M / L$, meeting at $N$ and $O$ the paralled to $G H$ drawn through $M$. 'Then

$$
G L=L I T=L . M \text {. }
$$

Hence.$V L$ and $O L$ are equilateral parallelograens, on them fore, by what has been already provel, the foree $M / C$ may 3 . rezarded as the resultant of $M / N$ and $M / L$, and the fror $M / I$ as the resultant of $M T O \mathrm{aml} M$. Henoe we may subetiuss for $M G$ and $M H$ the forces $M N, M O$, and the two fimes $M T$; $M L$ and $M O$, since they are equal and opposite, dearoy enely other, and we have remaining the two foros $M 4$. wMich tosertier give a force represented in magnitude and dinetis by $M / K$.

Seomills, suprose the angle $A M B$ not a rizhit angle. Through $G$ and $\|$ draw $C F F$ and $I T \%$ perpendicular to the diagunal $3 I K$, and $G N$ and $M O$ parallel to this straight line. Thwogh A/Araw X.MO at right angles is $M K$. Then we hare GE: $I I K$. As we lave already shewa. the furce $M / G$ may be replaced by $M / N$ and $M T E$, and the force $M / I T$ by $M / D$ and $M F$. Sinee $M / N$ and $M O$ are egual and ouproite, they will deatroy each other, and $M F$ and $M E$ remain; since $M E=K E$, we have $M K$ as the resultant in magnitude and direction of $M G G$ and $M / H$.

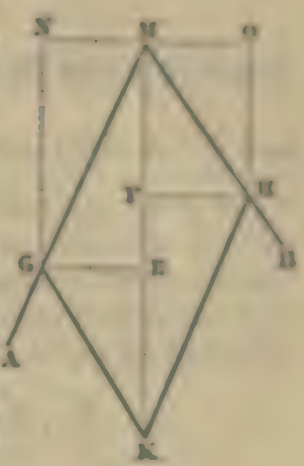

Hence the Porallelegram of Firces is completels porsed.

36. A prof of the Parallelogram of Forres has Lers

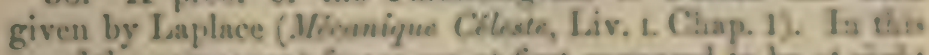
proof the component forces are at first amppoed to te at riplt angles; the magnitule of their resultant is then detranisod and afterwards its direction. The first part of the poot is s. simple, that it may to conveniently introdnard beres is is substantially as follows. Let $x$ and $y$ denote iwo foros wives are inclined at a right angle, and let g denote thair rosthast. we propose to find the value of a. It is obrieus that if tha components instead of being $x$ and $y$ were $2 x$ atad $2 y$ mopes. 
tively, the resultant would be $2 z$ and would have the same direction as before; so if the components were $3 x$ and $3 y$ respectively, the resultant would be $3 z$ and would have tho same direction as before; and so on. We may therefore assume conversely, that if the inclination of the resultant to each component remains unchanged, the ratio of each component to the resultant will also remain unchanged. Now consider the force $x$ as the resultant of two forces $x^{\prime}$ and $x^{\prime \prime}$, of which $x^{\prime}$ is in the direction of $z$, and $x^{\prime \prime}$ is at right angles to that direction. Then by the principle just assumed, we have

so that

$$
\frac{x^{\prime}}{x}=\frac{x}{z} \text {, and } \frac{x^{\prime \prime}}{x}=\frac{y}{z} ;
$$

$$
x^{\prime}=\frac{x^{2}}{z} \text {, and } x^{\prime \prime}=\frac{x y}{z} \text {. }
$$

Similarly $y$ may be resolved into $\frac{y^{2}}{z}$ along the direction of $z$ and $\frac{y x}{z}$ at right angles to that direction. Thus the forces $x$ and $y$ are equivalent to four forces, two in the direction of $z$ and the other two at right angles to that direction; the latter two are equal in magnitude and opposite in direction, so that they comteract each other; hence the resultant of the former two must be equal to $z$. Thus

$$
\frac{x^{2}}{z}+\frac{y^{2}}{z}=z ; \text { therefore } z^{2}=x^{2}+y^{2} \text {. }
$$

We shall now give some simple propositions which will serve to exemplify and illustrate the principles of the present Chapter.

I. $\triangle B C$ is a triangle; $D, E, F$ are the middle points of the sides $B C, C A, A B$ respectirely: shew that forces represented by the straight lines $A D, B E, C F$ will be in equilibrium.

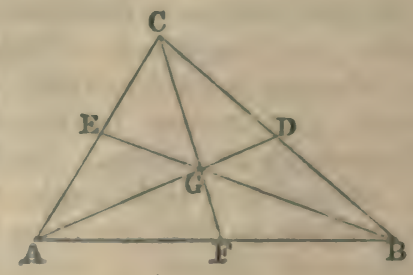


It is known that the straight lines $A D, B E, C F$ mies at a point: see Apjendix to Euclid. Iat $G$ denete this pisat. The three forces may be supponed to act at $G$.

Since $D$ is the middle point of $B C$, the parallelegran $d e$. seribed on $A B$ and $A O$ as adjacent sides wifl have a diagental in the direction $A D$; bence twice $A D$ will reprewst the resultant of two forees represented by $A B$ and $A C$. An conversely the force represented by $A B$ may be reasind inte two forces represented by half IB and hale $A C$. Kimilarty the force $B E$ may be resolved into half $B Q$ and lialf $B A$; and the force $C F$ may be resolved into half $C A$ and half $C I S$.

But the force half $A B$ is equal and opposite to the forse half $B A$; and so ou. Thus, finally, the foros $A D, E E, C F$ are in equilibrium.

II. In the figure of the proceding proposition firms me presented by the straight lines $Q A, Q B, Q C$ will be in equilibrium.

The resultant of the forces $G B$ and $G C$ acts aloog $G D$. If then there is not equilibrium the three foress $Q . i, G B$, (i) $C$ have a resultant acting eicher from $A$ towanls $D$ of forca $D$ towarls $A$, that is in the straight line $A D$. Bhat is the same way it may be shewn that if the fores GA, GR, GO nre not in equilibrium their resultant must act in the stnighte line $B E$, and also in the straight line $C F$. But it ia imnose:We that the resultant can act in three differnt stra: glat lines.

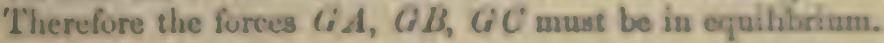

As the stndent is probably aware, it may be shewn by Geometry that $A G$ is equal to twice $Q D_{i}$ and thas the poosent theorem may be established directly; bat we have unod the methed here given for the parpose of illnstrating mechanieal principles. Wo may observe that we have ties by the aid of mechanical principleg, in fact, demonstrated thas $A G=2 G D$; for the resultant of $O B$ and $G C$ is reprosented by twice $G D$.

Since $A D=3 C D, B E=3 C F$, and $C F=3 Q P$, the frors $A D, B E, O F$ have the sume relative prepertion as the forces $G D, G E . G F$; so that the first proponition may bo dedword immediately from the secoml. 
III. Forces act at the middle points of a triangle, in the plane of the triangle, at right angles to the sides and respectively proportional to the sides: shew that if they all act inwards or all act outwards they will be in equilibrium.

The directions of the forces mect at a point, namely, the centre of the circle which circumscribes the triangle. And the angle between the directions of two forces is the supplement of the angle between the corresponding sides. Thus each force is as the sine of the angle between the other two. Ilence by $\Lambda$ rt. 19 the forces are in equilibrium.

IV. Forces act at the angular points of a triangle along the perpendiculars drawn from the angular points on the respectively opposite sides; and the forces are respectively proportional to the sides: shew that the forces will be in equilibrium.

It is known that the perpendiculars meet at a point: sce Appendix to Euclid. Hence by the preceding proposition the forces are in equilibrium.

V. $A B C$ is a triangle; $H, I, K$ are points in the sides $B C, C A, A B$ respectively such that

$$
\frac{B H}{H U}=\frac{C I}{I A}=\frac{A R}{K B} \text {. }
$$

Shew that if forces represented by $A H, B I, C K$ act at a point they will be in equilibrium.

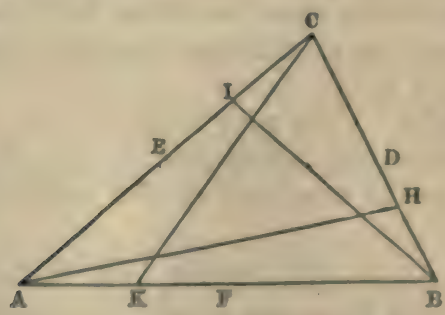

Let $D, E, F$ be the middle points of the sides; and suppose $A D, B E$, and $C F$ to be drawn. 
The force $A I I$ may be pesalsed into the faress $A D$. DUI, the force $I I$ inte the forers $I E E_{,} E I$; and the forve $C K$ ins the forces $C F, F K$. See Art. 20.

The forces $A D, B E$; $C F$ are in egruilitirium by the fint proposition.

And we have from the hypothesis as to $I I, I, K$,

$$
\frac{D I I}{B C}=\frac{E I}{U A}=\frac{F K}{A B}
$$

so that the forces DIT, EI, FK are proportional to the ailes of the triangle $A B C$ : and they are therefure in eguililatem by Art. 21 it they act at a point.

- Hence if the forees $A I I, B I, C K$ act at a point they are in equilibrium.

The straight lines $A I, D I, C K$ by thrir interwotbers fom a triangle; and therefore by Art. 19 the sides of this trias zlo are freportional to the forces. Hence we arrive by medased principles at the following geometrical restle tha sites of the triangle formed by the intersections of $A I I, B I, C K$ are propurtional to $A H, B I, C K$ respectively.

VI. $A, B, C$ are three prints on the cireumfenenes of a circle; forees act along $A B$ and $B C$ inversely progornianal is these straight lines in magnitude: shew that the resuliame acts along the tungent at $B$.

Denote the forees by $\frac{\mu}{A B}$ and $\frac{\mu}{B C}$ respectively. Fiewlve them at right angles to the tangent at $B$; thus we sibsin by Euclid, 1II. 32,

$$
\frac{\mu}{A B} \sin A C B-\frac{\mu}{C B} \sin C A B
$$

and this is rero, since

$$
\frac{C B}{A B}=\frac{\sin C A B}{\sin A C B^{\circ}}
$$

Hence the resultant must act along the tangent at $B$. 
VII. If one of two forces be given in magnitude and position, and also the direction of their resultant, the locus of the extremity of the straight line representing the other furce will be a straight line.

Let $a$ and $r$ denote the magnitudes of two forces; suppose the former to make an angle a with the direction of the resultant, and the latter an angle $\theta$.

Then, resolving along the straight line which is at right angles to the direction of the resultant, we have

$$
a \sin \alpha-r \sin \theta=0 \text {. }
$$

Now $a$ and $\alpha$ being given, while $r$ and $\theta$ are variable, this equation represents a straight line which is parallel to the direction of the resultant, and at a distance $a \sin \alpha$ from it. See Conic Sections, Chap. II.

VIII. From any point within a regular polygon perpendiculars are drawn on all the sides of the polygon: shew that the direction of the resultant of all the forces represented by these perpendiculars passes through the centre of the circle circumscribing the polygon, and find the magnitude of the resultant.

Let $p$ denote the perpendicular from the centre on a side, $c$ the distance of the point at which the forces act from the centre, $\alpha$ the angle which this distance makes with a fixed straight line which coincides with the perpendicular from the centre on a side, $n$ the number of sides in the polygon; and let $\beta=\frac{2 \pi}{n}$.

Then the magnitude of the $m^{\text {th }}$ force may be dennted by $p-c \cos (m \beta-\alpha)$, and the direction of this force will make an angle $m \beta$ with the fixed straight line.

Hence the resolved parts of the forces parallel to the fixed straight line, and at right angles to it, will be respectively, $\Sigma\{p-c \cos (m \beta-\alpha)\} \cos m \beta$ and $\Sigma\{p-c \cos (m \beta-\alpha)\} \sin m \beta$, where $\Sigma$ denotes a summation to be taken with respect to $m$ from $m=1$ to $m=n$. 
Also $\cos (m \beta-\alpha) \cos m \beta=\frac{1}{2}[\cos (2 m, \beta-\alpha)+\cos 3]$.

$$
\cos (m \beta-\alpha) \sin m \beta=\frac{1}{2}(\sin (2 m \beta-a)+\sin a) .
$$

Then effocting the summation, (wre Trigonomedry, Chap. xx:s) we obtain for the resolved parts,

$$
-\frac{n c}{y} \cos \alpha \text { and }-\frac{m c}{2} \sin \alpha .
$$

Hence, with the notation of Art. 22,

$$
\tan a=\tan a, \quad B=\frac{\pi}{2} \text {. }
$$

The former equation shews that the direction of the raste. ant evinciles with the atraight line which joins the entre $t$ the proint at which the furres act; and the latter ergation determines the magnitude of the resultant.

I.C. Suppose three forces $\boldsymbol{P}, Q, \mathbb{R}$ to act at a point $0, \mathrm{san}$ to he in equilibrium; let a circle be described with $O$ a centre, and suy radius, entting the directions of the firses at the points $A, B, C$ respectively: then shall $P, Q, I S$ be respectively proportional to the areas of the triangles $O B C, O C A, O A B$.

This follows at once from Art. 19, since the area of a triangle is expressed by half the product of two sides lista ther sine of the included angle.

X. Suppose four forces $P, Q, R, S$ to act at a poiat $O$. and to be in equilibrium; let a sphere he described wis 0 as centre, and any radius, cutting the directions of the faroes as the points $A, B, C, D$ respectively: then ahail $P, Q, I, S$ be respectisely proportional to the volumes of the pyramids OBCD, OCDA, ODAB, OABC.

Take $O$ as the origin of a set of rectangular axes, letar, $y, 5$

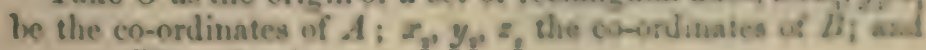
80 on. Then, by Art. a7,

$$
\begin{aligned}
& P x_{1}+Q x_{0}+R x_{0}+S r_{0}=0 \text {, } \\
& I_{y_{1}}+Q_{y_{0}}+I_{y_{0}}+\Sigma_{z_{0}}=\theta_{0} \\
& P_{z_{1}}+Q z_{0}+k z_{1}+S_{z_{1}}=0 \text {. }
\end{aligned}
$$


Hence, eliminating $Q$ and $R$, we shall obtain

$$
P=S \frac{x_{6}\left(y_{3} z_{2}-y_{2} z_{2}\right)+y_{4}\left(z_{3} x_{2}-z_{2} x_{3}\right)+z_{4}\left(x_{3} y_{2}-x_{2} y_{3}\right)}{x_{1}\left(y_{2} z_{3}-y_{3} z_{2}\right)+y_{1}\left(z_{2} x_{3}-z_{2} x_{2}\right)+z_{1}\left(x_{2} y_{3}-x_{3} y_{2}\right)} .
$$

Hence, by the aid of the expression for the volume of a pyramid given in works on Analytical Geometry of three dimensions, we have

$$
\frac{P}{S}=\frac{\text { volume of pyramid } O B C D}{\text { volume of pyramid } O A B C} \text {. }
$$

Similarly we obtain the value of $\frac{Q}{S}$ and of $\frac{R}{S}$.

\section{EXAMPLES.}

1. Two forces $P$ and $Q$ have a resultant $R$ which makes an angle $\alpha$ with $P$; if $P$ be increased by $R$ while $Q$ remains unchanged, shew that the new resultant makes an angle $\frac{a}{2}$ with $P$.

2. Two forces in the ratio of 2 to $\sqrt{ } 3-1$, are inclined to each other at an angle of $60^{\circ}$; what must be the direction and magnitude of a third force which produces equilibrium?

Result. The required force must be to the first of the given forces as $\sqrt{6}$ to 2 ; and its direction produced makes an angle of $15^{\circ}$ with that force.

3. The resultant of two forces $P$ and $Q$ is equal to $Q \sqrt{ } 3$, and makes an angle of $30^{\circ}$ with $P$; find $P$ in terms of $Q$.

Rrsult. Either $P=Q$ or $P=2 Q$; in the former case the angle between $P$ and $Q$ is $60^{\circ}$, in the latter $120^{\circ}$.

4. If $D, E, F$ be the middle points of the sides of the triangle $A B C$ and $O$ any other point, shew that the system of forces represerted by $O D, O E, O F$ is equivalent to that represented by $O A, O B, O C$. 
5. The resuleant of two furcen in 1011 s, one of them is equal to $81 \mathrm{lb}$, and the dircetion of the cethes is in finod so the resultant at an angle of $36^{\circ}$. Find the angle betwern the two furees.

$$
\text { Liesuld. } \operatorname{Sin}^{-1} \frac{5}{16}(10-2 \sqrt{3})^{1} \text {. }
$$

6. The resultant of two forces $P, Q$, neting at an angle $\rho$. is eyual to $(2 m+1) \sqrt{ }\left(P^{\mu}+Q^{n}\right)$; when they act at an angt: $\frac{1}{2 \pi} \pi-\theta$, it is equal to $(2 m-1) \sqrt{ }\left(P^{n}+\bar{Q}\right)$; shew that $\tan \theta=\frac{m-1}{m+1}$.

7. Two forces $F$ and $F$ acting in the diagonals of a paralielingram keep it at rest in such a position that one of is elges is hurizutal, shew that

$$
F \sec \alpha^{\prime}=F^{\prime} \sec \alpha=W \operatorname{cosec}\left(z+a^{\prime}\right),
$$

where $W$ is the weight of the parallelogram, a and $a$ the angles between its diagonals and the horizoutal sile.

8. If a particle be placed on a splere, and be actel on by three fores represented in magnitude and diroction by three chords mutually at right angles drawn throngth te particle, it will remain at rest.

9. Three forces $P, Q, R$ acting on a point and kerping it at rest are represented by straight lines drawn from that point. If $P$ be given in magnitude and direction, and $Q$ ia magnitude only, find the locus of the extremity of the lins which represents the third force $\mathbb{R}$.

\section{Result. A sphere.}

10. A circle whose plane is vertical has a centre of corstant repulsive force at one extremity of the horizontal thameter; find the position of equilibriam of a particle sithin the circle, the repulsire force being equal to the waight of the particle.

Result. The straight line joining the partide with the centre of the circle makes an angle of $60^{\circ}$ with the honians. 
11. A particle is placed on a smooth square table whose side is a nt distances $c_{1}, C_{y}, C_{3}, C_{4}$ from the corners, and to it are attached strings passing over smonth pullies at the corners and supporting weights $P_{2}, P_{2}, P_{3}, P_{4}$; shew that if there is equilibrium,

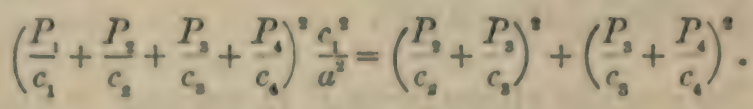

Shew also that

$$
\left(\frac{P_{3}}{c_{1}}+\frac{P_{3}}{c_{3}}+\frac{P_{3}}{c_{3}}+\frac{P_{4}}{c_{4}}\right) \frac{c_{1}^{2}-c_{3}^{2}}{a^{3}}=2\left(\frac{P_{3}}{c_{3}}-\frac{P_{1}}{c_{1}}\right) .
$$

12. Two small rings slide on the are of a smooth vertical circle; a string passes through both rings, and has three equal weiglits attached to it, one at each end and one between the rings; find the position of the rings when they are in equilibrium. The rings are supposed without weight.

Result. Each of the rings must be $30^{\circ}$ distant from the highest point of the circle.

13. The extremities of a string without weight are fastened to two equal heavy rings which slide on smooth fixed rods in the same vertical plane and equally inclined to the vertical: and to the middle point of the string a weight is fastened equal to twice the weight of each ring; find the position of equilibrium and the tension of the string.

If the point to which the weight is fastened be not the middle point of the string, shew that in the position of equilibrium the tensions of its two portions will be equal.

14. A light cord with one end attached to a fixed point passes over a pully in the same horizontal line with the fixcd point and supports a weight hanging freely at its other end. $A$ heary ring being fastened to the cord in different places between the fixed point and the pully, it is required to find the locus of its positions of equilibrium. If the weight of the ring be small compared with the other weight, the locus will be approximately a parabola. 
15. If two forces acting along choods of a circle are is versely proportional to the lengtiss of the chonde their mat? tunt will pass through one or other of the points of intarnestioes of straight lines drawn through the extremitien of the chonds.

16. A particle rests on an ellipse acted an by farver $\lambda x^{\circ}$. $\mu y^{\circ}$, parallel to the axes of $x$ and $y$ respectively; find it position of equilibrium. Explain the came in which $n=1$.

17. A particle is placed on the outer aurfince of a movels fixed sphere and is acted on by a fixed centre of force lying vertically above the centre of the sphere, at a distanee e frum if snd attracting directly as the distance. Shew that the partiele will rest on any part of the sphere if the weight of the particle equals the attraction on it by the fised cestre of forcer when at a distance $c$ from it.

18. A particle is placed on the surface of $m n$ ellipsond in the eentre of which is resident an attractive forem: determitas the direction in which the particle will begin to move.

19. Find the point on the surface $\frac{x^{2}}{a^{3}}+\frac{y^{2}}{b}+\frac{z^{2}}{e^{3}}=1$, wher a particle attracted by a force to the origin will reat in ergilibrium.

20. $A B C D$ is a quadrilateral inseribed in a cirele asd forces inversely propurtional to $A B, B C, A D, D C$ act al ho the sides in the directions indicated by the leuters: shew that their resultant acts along the straighe line jouning the ister. section of the diagonals with the infersection of the tangents to the circle at $B, D$. 


\section{CHAPTER III.}

RESULTANT OF TWO PARALLEL FORCEB. COUPLES.

37. To find the magnitude and direction of the resultant of two parallel forces acting on a rigid body.

Let $P$ and $Q$ be the forces; $A$ and $B$ their points of ap-

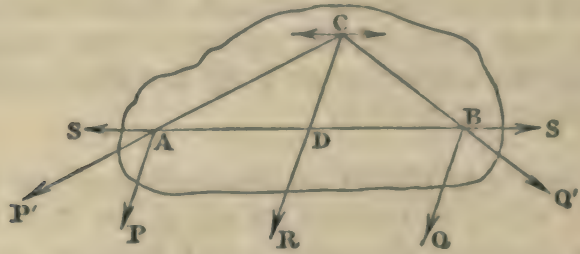

plication: let $P$ and $Q$ act in the same direction, making angles $\alpha$ with $A B$. 'The effect of the forces will not be altered if we apply two forces equal in magnitude and acting in opposite directions along the straight line $A B$. Let $S$ denote each of these forces, and suppose one to act at $A$ and the other at $B$.

Then $P$ and $S$ acting at $A$ are equivalent to some force $P^{P}$ acting in some direction $A P^{\prime}$ inclined to $A P(\Lambda \mathrm{rt} .17)$; and $Q$ and $S$ acting at $B$ are equivalent to some force $Q$ acting in some direction $B Q^{\prime}$ inclined to $B Q$.

Produce $P^{\prime} A, Q^{\prime} B$ to cut each other at $C$, and draw $C 7$ ) parallel to $A P$, meeting $A B$ at $D$; suppose $C$ rigidly connected with $A B$.

Transfer $P^{\prime}$ and $Q^{\prime}$ to $C$ (Art. 11), and resolve them along $C D$ and a straight line parallel to $A B$; the latter parts will each be equal to $S$ but act in opposite directions, and the sum of the former is $P+Q$. Hence $R$, the resultant of $P$ and $Q$, $=P+Q$ and acts parallel to $P$ and $Q$ in the straight line $C D$. We shall now determine the point where this straight line cuts $A B$. 
The sides of the triangle $A C D$ are parallel to the directiona of the forces I, S, P"; therefore by Art. 19

$$
\frac{P}{S}=\frac{C D}{11 A} \text {, and similarly } \frac{S}{Q}=\frac{D B}{C D} \text {; }
$$

therefore $\frac{P}{\varphi}=\frac{D B}{D A}=\frac{a-x}{x}$, if $A B=a$ and $A D=x$;

therefore

$$
\frac{x}{a}=\frac{Q}{P+Q}
$$

this determines the point $D$ through which the directine of the resultant passes. It will be otserved that $A B$ is dividmi at $D$ into segments which are inversely as the forces at $A$ and B respectively.

If the force $P$ act in a direction oppenite to that of $Q$

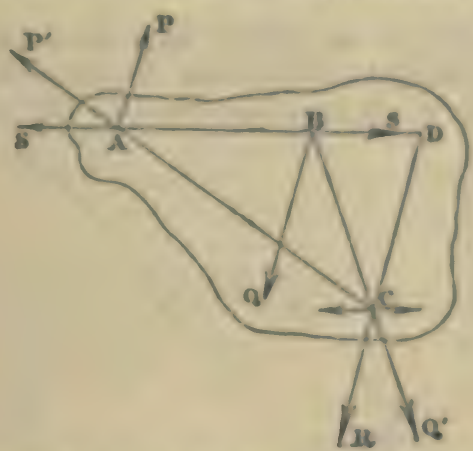

a similar process will lead us to

$$
R=Q-P, \text { and } \frac{x}{a}=\frac{Q}{Q-P}
$$

which may be derived from the furmule of the preneding case by changing $P$ into $-P$.

It will be observed that $A B$ produced is divided as $D$ into segments which are inversely as the furees at $A$ and $B$ ro spectively. 
38. The point $D$ possesses this remarkable property: that however $P$ and $Q$ are turned about their points of application $A$ and $B$, their directions remaining parallel, $D$ determined as above remains fixed. This point is in consequence called the centre of the parallel forces $P$ and $Q$.

39. If $P=Q$ in the second case of $A$ rt. 37 , then $R=0$ and $x=\infty$, a result perfectly nugatory. It shews us that the method fails by which we have attempted to compound two equal and opposite parallel forces. In fact the addition of the two forces $S$ still gives, in this case, two equal forces parallel and opposite in their directions.

Such a system of forces is called a Couple.

We shall investigate the laws of the composition and resolution of couples, since to these we shall reduce the composition and resolution of forces of every description acting on a rigid body.

40. From Art. 39 we might conjecture that two equal forces acting in parallel and opposite directions do not admil of a single resultant, which may be shewn as follows.

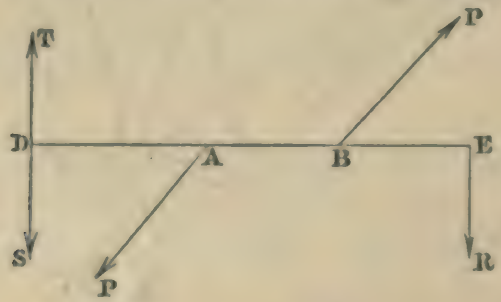

Suppose, if possible, that the single force $R$ will maintain equilibrium with two forces, each denoted by $P$, acting in parallel and opposite directions.

Draw a straight line meeting at $A$ and $B$ the directions of the forces $P$, and that of $R$ at $E$. Make $A D=B E$, and apply at $D$ two forces $T$ and $S$ each $=R$ and parallel to $R$ but in opposite directions; this will not disturb the equilibrium. Hence the five forces $R, P, P, S, T$ are in equilibrium. But since $P, P$ and $I C$ form a system in equilibrium, so by sym- 
metry do $P, P$ and $T$. Hence if we remove the last there we shall not disturb the equilitrium, and we acovedingly hare $\boldsymbol{R}$ and $s$ left maintaining equilitrium. But this is ctorieusly impossible, since they act in the same direction. IIewer the two parallel forces f' cannot be balanced by a single furor, and therefore do not admit of a single resultant.

41. A couple consists of two equal forces acting in parallet and opposite directions.

The arm of a couple is the perpendicular distance between the directions of its furces.

The moment of a couple is the product of cither of its fornes into the perpendicular distance between therw.

The axis of a couple is a straight line perpendicular to the plane of the couple and proportional in length to the moment.

Two couples in the sane plane may differ with respect to direction. For suppose the middle point of the arm of a couple to be fixed, and the arm to move in the direction in which the two forces of the couple tend to urge it; there are swo different diroctions in which the arm may rotate. Sep. pose a perpendicular drawn to the plane of the couple through the middle point of its arm, so that when an observer is placed along this straight line with his feet againat the plane, the rotation which the forces give to the arm appears to ake place from lefi to righe; the perpendicular so drawn we shall ake for the axis of the couple.

We shall now give three propositions shewing that the effect of a couple is not altered when certain changes are male with respect to the couple. It is to be supposed in all these propositions that a rigid binly is in equifibrims under the action of certain forces, including an assigned couple; and is is ghewn that then the equilitorium will not bo disturbed by stre specified changes with respect to the couple.

42. The effect of a couple is not aleered if its arm le turmat through any ungle alout one extremoisy in the plane of the couple. 
Let the plane of the paper be the plane of the couple, $A B$ the arm, and $A B^{\prime}$ its new position; the forces $P_{1}, P_{\text {, are equal }}$

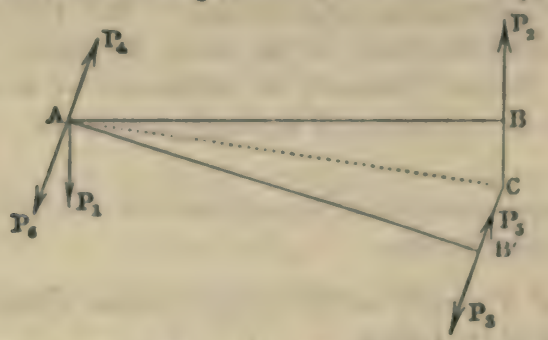

and act on the arm $A B$. At $B^{\prime}$ and $A$ let the equal and opposite forces $P_{8} P_{8}, P_{4} P_{6}$. each equal to $P_{1}$ or $P_{8}$ be applied, acting at right angles to $A B^{\prime}$; this will not affect the action of $P_{1}$ and $P_{2}$.

Let $B P_{2}, B^{\prime} P_{3}$ meet at $C$; join $A C ; A C$ manifestly bisects the angle $B A B^{\prime}$.

Now $P_{2}$ and $P_{3}$ are equivalent to some force in the direction $C A$, and $P_{1}$ and $P_{4}$ are equivalent to an equal force in the direction $A C$. Therefore $P_{1}, P_{2}, P_{3}, P_{4}$ are in equilibrium with each other; therefore the remaining forces $P_{5}, P_{8}$ acting at $B, A$ respectively produce the same effect as $P_{3}, P_{1}$ acting at $B, A$ respectively. Hence the proposition is true.

We may now turn the arm of the couple through any angle about $B^{\prime}$; and by proceeding in this way we may transfer the couple to any position in its own plane.

43. The effect of a couple is not altered if we transfer the couple to any plane parallel to its own, the arm remaining parallel to itself.

Let $A B$ be the arm, $A^{\prime} B^{\prime}$ its new position parallel to $A B$.

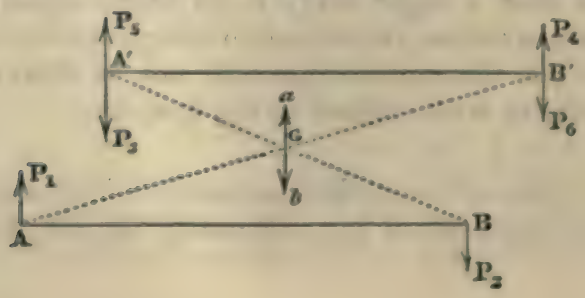




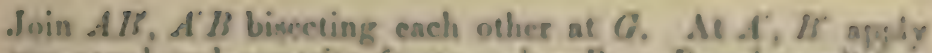
two ejual and oppwite forces eacii $=P$, or $P$, and juarallet in them: and let these forces be $P_{0}, P_{0}, P_{0}, P_{0}$ this will not alieg the effect of the couple.

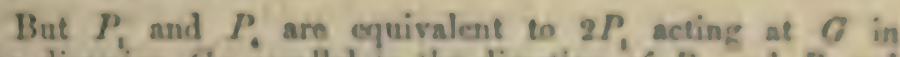
the direction Cia parallel to the direction of $P_{1}$, and $P_{\text {, and }}$ $P$, are equiralent to $2 P_{\text {, acting at }} G$ in the opjposite dirnetion $G b$.

Hence $P_{1}, P_{2}, P_{0}, P_{\text {, are }}$ in equilibrium with each ether: therefore the remaining forces $B$, and $P$, acting at $A$ and $B$ respectively produce the same eflect as $l_{i}$ and $\mathcal{P}_{\text {, aeting }}$ at $A$ and $B$ respectively. Hence the propusition in true.

44. The effect of a comple is mot altered if me roplane if by another couple of veloich the mament is the same; the plane romaining the same and the arms being in the same otraight line and haring a common extremily.

Let $A B$ be the arm; let $P$. $P$ be the forces, and suppose $P=Q+R$; let $A B=a$, and let the new arm $A C=b$; at $C$ apply two opposite forces each $=Q$ and parallel to $P$; this will not alter the effect of the cuuple.

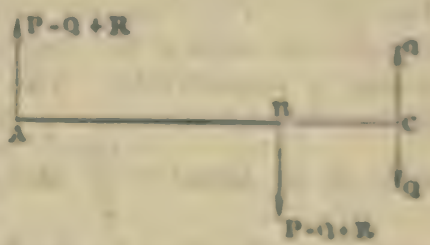

Now $R$ at $A$ and $Q$ at $C$ will balance $Q+R$ at $R_{\text {s }}$

$$
\begin{gathered}
\text { if } A B: B C: Q: R_{,}(A r t .3 i), \\
\text { or if } A B: A C:: Q: Q+R_{i} \\
\text { that is, if } Q . B=P \cdot a \text {; }
\end{gathered}
$$

we have then remaining the couple $Q . Q$ acting on the arm $A C$. Hence the couple $P, P$ acting on $A B$ miny be milacol by the couple $Q, Q$ acting on $A C$, if $Q . B=P$. $a$, that is, if their moments are the same.

45. From the last three Articlea it appeans that, withrut altering the effect of a couple, we may cliange is inte swother 
of equal moment, and transfer it to any position, either in its own plane or in a plane parallel to its own. The couple must remain unchanged so far as concerns the direction of the rotation which its forces would tend to give the arm, supposing its midule point fixed as in Art. 41. In other words, the straight line which we have called the axis, measured as indicated in that Article, must always remain on the same side of the plane of the couple.

46. We may infer from Art. 41 that couples may be measured by their moments. Let there be two couples, one in which each force $=P$, and one in which each force $=Q$, the arms of the couples being equal; these couples will be in the ratio of $P$ to $Q$. For suppose, for example, that $P$ is to $Q$ as 3 to 5 ; then each of the forces $P$ may be divided into 3 equal forces and each of the forces $Q$ into 5 such equal forces. 'Then the couple of which each force is $P$ may be considered as the sum of 3 equal couples of the same kind, and the couple of which each force is $Q$ as the sum of 5 such equal couples. The effects of the couples will therefore be as 3 to 5 . Next, suppose the arms of the couples unequal, and denote them by $p$ and $q$ respectively. 'The couple which has cach of its tores $=Q$ and its arm $=q$ is equivalent to a couple having each of its forces $=\frac{Q q}{p}$ and its arm $=p$, by Art. 44. The couples are therefore by the first case in the ratio of $P$ to $\frac{Q_{q}}{p}$, that is of $P_{p}$ to $Q q$.

47. With respect to the effect of a couple, we may observe that it is shewn in works on rigid dynamics that if a couple act on a free rigid body it will set the body in rotation about an axis passing through a certain point in the body called its centre of gravity, but not necessarily perpendicular to the plane of the couple.

48. To find the resultant of any number of couples acting on a body, the planes of the couples being parallel to each other.

First. suppose all the couples transferred to the same plane (Art. 43); next, let them be all iransferred so as to have 
their arms in the name straight line, and one extrrnigy coenmon (Art. 42); and lasely, let them le refibeed by other couples haring the same arm (Art. 4t).

$$
\begin{gathered}
\text { Thus if } P, Q, R, \ldots \ldots . . \text { be the foroes, and } \\
a, b, c, \ldots \ldots . . \text { be their arms, }
\end{gathered}
$$

we shall have them replaced by the following firas (oupposing Q the comnon arm),

$$
P \cdot \frac{a}{a}, Q \cdot \frac{b}{a}, \quad R \cdot \frac{a}{a}, \ldots \ldots \ldots \text { acting on the arru-a. }
$$

Hence their resultant will be a couple of which each foroe equals

$$
P \cdot \frac{a}{a}+Q \cdot \frac{b}{a}+B \cdot \frac{c}{a}+\ldots \ldots \ldots .
$$

and the arm $=a$,

or of which the moment eruals

$$
P \cdot a+Q .8+R . \odot+\ldots \ldots \ldots
$$

Hence the moment of the resultant couple is equal to the sum of the moments of the original cutuples.

If one of the conples, as $Q, Q$, act in a dimection egpraive fo the couple $P, P$, then the furce at each extremity of the armo of the resultant couple will be

$$
P \cdot \frac{a}{a}-Q \cdot \frac{b}{a}+16 \cdot \frac{c}{a}+\ldots \ldots \ldots,
$$

and the moment of the resultant couple wil! be

$$
\boldsymbol{P} \cdot \boldsymbol{a}-\boldsymbol{Q} . \boldsymbol{B}+\boldsymbol{R} . \boldsymbol{0}+\ldots \ldots \ldots,
$$

or the algebraical sum of the moments of the original couples: the moments of thase conples which tend in the dirrising opposite to the couple $P, P$ teing reckoned negalive. 
49. To find the resultant of two couples not acting in the sane plane.

Let the planes of the eouples intersect in the straight line

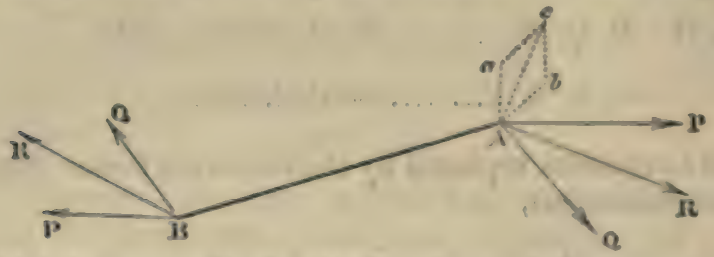

$A B$, which is perpendicular to the plane of the paper, and let the couples be referred to the common arm $A B$, and let their furces thus altered be $P$ and $Q$.

In the plane of the paper draw $A a, A b$ at right angles to the planes of the couples $P, P$ and $Q, Q$; and equal in length to their axes.

Let $R$ be the resultant of the forces $P$ and $Q$ at $A$, acting in the direction $A R$; and of $P$ and $Q$ at $B$, acting in the direction $B R$.

Since $A P, A Q$ are parallel to $B P, B Q$ respectively, therefore $A R$ is parallel to $B R$.

Hence the two couples are equivalent to the single couple $R, R$ acting on the arm $A B$.

Draw $A c$ perpendicular to the plane of $R, R$, and in the same proportion to $A a, A b$ that the moment of the couple $R, R$ is to those of $P, P$ and $Q, Q$ respectively. Then $A c$ is the axis of $R, R$. Now the three straight lines $A a, A c, A b$ make the same angles with each other that $A P, A R, A Q$ make with each other; also they are in the same proportion in which $A B . P, A B . R, A B . Q$ are; t'lat is in which $P, R, Q$ are.

But $R$ is the resultant of $P$ and $Q$; therefore $A c$ is the diagonal of the parallelogram on $A a, A b$ (see $A$ rt. 17).

Hence if two straight lines, having a common extremity, represent the axes of two couples, that diagonal of the parallelogram described on these straight lines as adjacent sides which passes through their common extremity represents the axis of the resultant couple. 


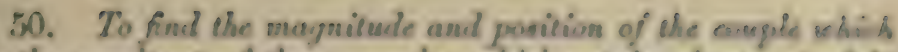
is the resultant of three couplas achich act in planes at rojh angles to each ocher.

Let $A B, A C, A D$ be the axen of the given couples lene fig. to Art. 21). Complete the parallelogram CB, and dram $A E$ the diagonal. Then $A E$ in the axis of the cutpple which is the resultant of the two couples of which the axes ane $A B, A C$. Complete the parallelogram $D E$, and draw $A F^{\circ}$ the diagonal. Then $A F$ is the axis of the couple which is the resultant of the couples of which the axes are $A E, A D$, or of those of which the axes are $A B, A C, A D$.

Now $A E^{2}=A B^{2}+A D^{2}=A B^{2}+A O^{2}+A D^{\circ}$.

Iat $G$ be the moment of the resultant erpiple; $L_{2}, M, N$ those of the given couples ;

therefore

$$
G^{\prime}=I^{3}+M^{\top}+N^{\top} ;
$$

and if $\lambda, \mu, v$ be the angles the axis of the resultant maken with those of the components,

$$
\cos \lambda=\frac{A B}{A b^{\prime}}=\frac{L}{Q^{\prime}} ; \cos \mu=\frac{M}{Q^{\prime}} ; \cos v=\frac{N}{Q^{\prime}} \text {. }
$$

51. Hence conversely any couple may be replaced by three couples acting in planes at right angles to each velier; their moments being $G \cos \lambda, G \cos \mu, G \cos v$; where $G$ in the moment of the given couple, and $\lambda, \mu, \nu$ the angles its axis makes with the axes of the three couples.

Thus couples follow, as to their composition and moulurisn, laws similar to those which apply to forcs, the aris of the cuuple corresponding to the direction of the furve and the moment of the couple to the intensity of the furve. Ilence for example, by Art. 29, the resiolsed part of a mosultant couple in any direction is equal to the sum of the resolred parts of the component couples in the same direction. 


\section{CHAPTER IV.}

RESULTANT OF FORCES IN ONE PIANE. CONDITIONS OF EQUILIBHIUM. MOMENTS.

52. To find the resultant of any number of parallel forces acting on a rigid body in one plane.

Let $P_{1}, P_{2}, P_{3} \ldots \ldots$ denote the forces. Take any point in

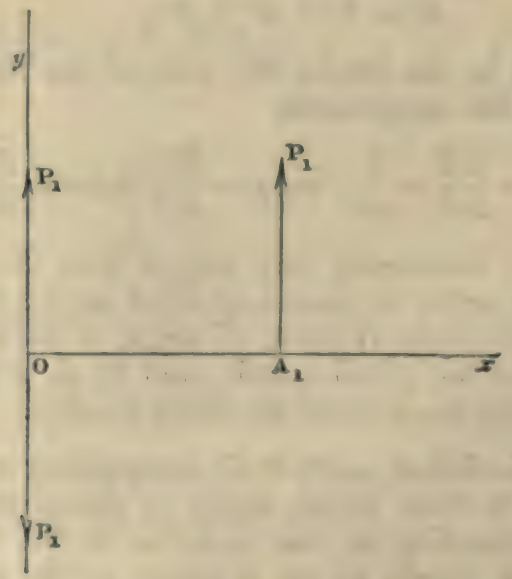

the plane of the forces as origin and draw rectangular axes $O x, O y$, the latter parallel to the forces. I Let $A_{i}$ be the point where $O x$ meets the direction of $P_{1}$, and let $O A_{1}=x_{1}$.

Apply at $O$ two forces each equal and parallel to $P_{1}$, in opposite directions. Thus the force $P_{1}$ is replaced by $P_{1}$ at $O$ along $O y$, and a couple of which the moment is $P_{1} . O A_{1}$, that is $P_{2} \cdot x_{1}$. 'Transform the other forces in a similar manner, 
nsing a similar notation, and the whale ayse-m will be rnterel

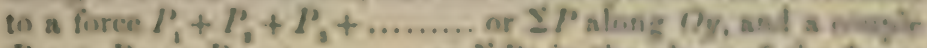

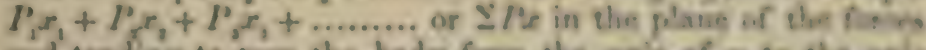
and tending to curn the body from the axis of a tis the as. of $y$.

53. To find the conditions of equilitrium of a syoerm of parallel forces acting on a rigid body in one plame.

A syefem of parallel forces ean be reduced in a single force and a couple. If neither of theer vanish equliksium is impossible. becauee a single force camnot nentralize a conple (Art. \$n!. If the single force aline vanish equilibriusn is impossible, beesnse there remains an unlatanoed couple. If the couple alone vanish ergulibrium is imposaible, becarser there remains an unbalanoed force. Henos for equilibrium it is necessary that both the force and the couple alvould vanish; that is

$$
\Sigma P=0 \text { and } \Sigma P=0 \text {. }
$$

54. The product of a fores into the perpendicular drawn upon its dirvetion from any point, is called the moment of the force with respect to that point. Hence the ousditoms of muilibrium which have just been obtained may bo thas enunciated:

A sys'em of parallel forces acling on a rigil Loly is ane

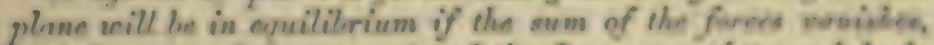
and the sum of the moments of the forces rensal an erigin in the plane also emmishes.

Conversely, if the forces are in cquilibrium theip sum mwet ranish, and also the sum of their moments round any origin in the plane.

The word sum must be underatoul a'gelimionlly. Fisors which act in one direction being consisterod positive, these which net in the opposite direction must lo ovesidered segartive. Also moments being considered positive when the oorresponding couples tend to furn the fraly in ene dirnction. they must be considered meratien when the correpondthe couples tend to turn the borly in the oppresite dirnetion. 
55. When the sum of the forces vanishes in $\Lambda$ rt. 52, the forces reduce to a couple. When $\Sigma P$ is not zero, the forces can be reduced to a single resultant. For if $\Sigma P x=0$, then $\Sigma P$ acting at $O$ is the single resultant. If $\Sigma P x$ be not $=0$, let the couple be transformed to one in which each of the forces is equal to $\Sigma P$, and consequently, by Art. 44, the arm is $\frac{\Sigma P x}{\Sigma P}$. Let $\Sigma P$ acting at $A$

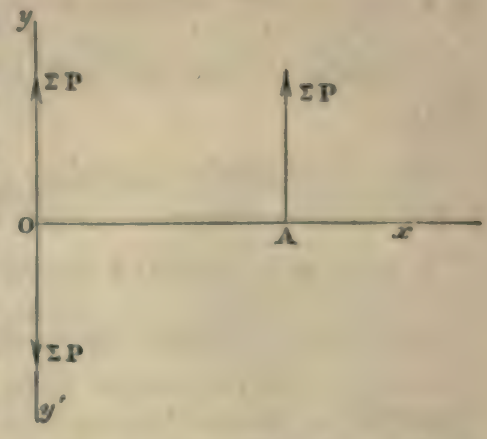
and $\Sigma P$ acting along $O y^{\prime}$ form this couple. The latter force is destroyed by the force $\Sigma P$ along $O y$. Hence the single resultant is $\Sigma P$ acting at $A$, that is, at a point the distance of which from $O$ is $\frac{\Sigma P x}{\Sigma P}$.

56. To find the resultant of any number of forces which act on a rigid body in one plane.

Let the system be referred to any rectangular axes $O x, O y$ in the plane of the forces.

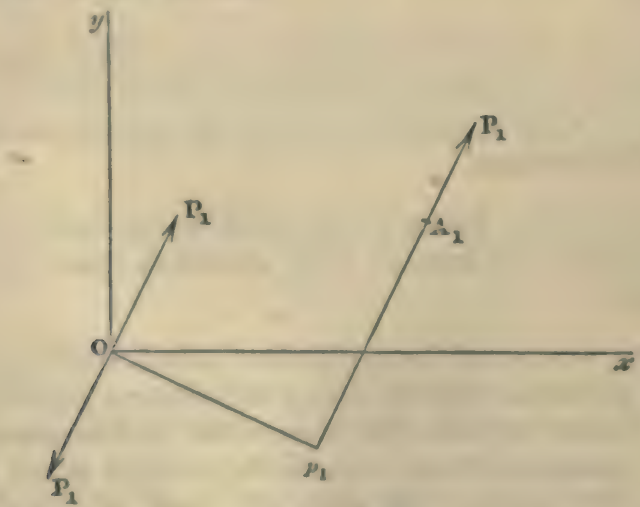

Let $P_{1}, P_{2}, P_{3}, \ldots \ldots$ denote the forces; $\alpha_{1}, \alpha_{2}, \alpha_{3}, \ldots .$. the angles which their directions make with the axis of $x$; 
let $x_{1}, y_{2}$ be the co-ordinates of the point of application of $P_{4}$ : let $x_{1}, y_{2}$ be those of the point of application of $P_{0}$, and $w$ on.

Let $A_{1}$ be the point of application of $P_{2}$. As 0 auppom two forces applied in opprite direction each ogual and parallel to $P_{1}$. Draw $O_{p}$ perpendicular to $P_{1} A_{1}$.

Hence $P_{3}$ acting at $A_{1}$ in equivalent in $P_{4}$ acting at $O$ and a couple of which $O_{B}$ is the arm and cach foris is $P_{2}$. which tends to turn the body from the axis of a to that of y. Now

$$
O_{p_{3}}=x_{3} \sin \alpha_{3}-y_{1} \cos \alpha_{3} \text {. }
$$

II ence the moment of the couple is

$$
P_{8}\left(x_{2} \sin \alpha_{1}-y_{1} \cos a_{2}\right) \text {. }
$$

The other forces may be similarly replaced. Henoe the system is equiralent to the forcess

$$
P_{1}, P_{3}, P_{2}, \ldots \text {... acting at } O \text {, }
$$

in directions parallel to thome of the original furces; and the couples of which the moments are

$$
\begin{aligned}
& P_{8}\left(x_{3} \sin a_{3}-y_{3} \cos a_{2}\right), \\
& P_{8}\left(x_{3} \sin a_{2}-y_{2} \cos a_{2}\right), \\
& P_{8}\left(x_{2} \sin a_{3}-y_{8} \cos a_{2}\right),
\end{aligned}
$$

acting in the plane of the foroes. It will be found that any one of the above expressions for the moments of the couples is positive or negative, according as that couple tends to tam the body from the axis of $x$ towanls that of $y$, of in the contrary direction.

Let $R$ be the resultant of the forces acting at 0 , let a la the angle which $R$ makes with the axis of $x$, and $Q$ the moment of the resultant couple; then (by Art $z$ )

$$
R \cos a=\Sigma P \cos \alpha ; \quad R \sin a=\Sigma P \sin \alpha ;
$$

and (by Art. 48)

$$
G=\Sigma P(x \sin a-y \cos \alpha) .
$$


If $P_{1} \cos \alpha_{1}=X_{1}$ and $P_{1} \sin \alpha_{1}=Y_{1}$, and a similar notation be used for the other forces, the above equations may be written

and

$$
\begin{gathered}
R^{n}=(\Sigma X)^{2}+(\Sigma Y)^{2} ; \tan a=\frac{\Sigma Y}{\Sigma X} ; \\
G=\Sigma(Y x-X y) .
\end{gathered}
$$

57. To find the conditions for the equilibrium of a system of furces acting on a rigid body in one plane.

Any system of forces acting in one plane may be reduced. to a single force $R$, and a couple whose moment is $G$. If neither $R$ nor $G$ vanish equilibrium is impossible, since a single force cannot balance a couple. If $R$ alone vanish equilibrium is impossible, because there remains an unbalanced couple $G$; if $G$ alone vanish equilibrium is impossible, because there remains an unbalanced force. Hence, for equilibrium we must have $R=0$ and $G=0$. Also $R=0$ requires that $\Sigma X=0$ and $\Sigma Y=0$.

Since $G$ is equal to the sum of the moments of the forces with respect to $O$, we may enunciate the result thus: $A$ system of furces acting in one plane on a rigid body will be in equilitivium if the sums of the resolved parts of the furces parallel to two rectangular axes in the plane vanish, and the sum of the moments round an origin in the plane also vanishes.

Conversely, if the forces are in equilibrium the sum of the resolved parts of the forces parallel to any direction will vanish, and also the sum of the moments of the forces round any origin.

58. If three forces acting in one plane maintain a rigit body in equilibrium their directions either all meet at a point or are all parallel.

For suppose two of the directions to meet at a point, and take this point for the origin; then the moment of each of these two forces vanishes, and the equation $G=0$ requires that the moment of the third force should vanish, that is, the third force must also pass through the origin. Hence, if any two of the forces meet, the third must pass through their point of intersection, which proves the proposition. This pro- 
poaition may also be eatablished without referring on Art St. For if two of the forees meet at a polnt, they may le anupoed both to act at that point and may be replacel ty tide so sultant acting at the same point; this mentant ast the thind force must keep the body on which thry act in equilikestes. ant must therefore be equal and oppositer that is thas thind furce must pass through the poist of intersoction of the first two.

59. If $R=0$ in Art. 36, the forves reduco to a couples if $I i$ be not $=0$, the forces can be reduced to a single rusiltart.

For if the couple $\vec{i}=0$, the resultant fore in $R$ meting at the origin. If the couple $G$ be not $=0$, let it he trassformed into one having each of ita forces $=I S$ and its arm coth-

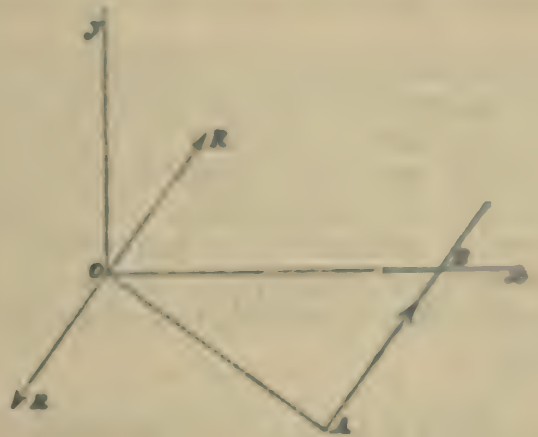

sequently $=\frac{C}{R}($ Art. H). Let this couple be tumed in its ows. plane, until one of its furces acts at the origin exactly eppesite to the furce $R$, which by hypothesis acts at the oritin. Hesce these forees drstroy each other and we have lent $R$ acting at the extremity of the arm $0 . A$, in a discetion iselined t5 the axis of $x$ at an angle $a$, found by the equation tan $a=\frac{\Sigma Y}{2 x}$ (Art. 56). If this direction meet the axis of $x$ at $B$, we hare

$$
O B=O A \operatorname{cosec} a=\frac{G}{J} \cdot \frac{R}{\Sigma}=\frac{a}{\Delta \lambda} .
$$


and the equation to the line of action of the single resultant is

or,

$$
\begin{gathered}
y^{\prime}=\frac{\Sigma Y}{\Sigma X}\left(x^{\prime}-\frac{G}{\Sigma Y}\right) ; \\
x^{\prime} \Sigma Y-y^{\prime} \Sigma X=\Sigma(Y x-X y),
\end{gathered}
$$

$x^{\prime}, y^{\prime}$ being the variable co-ordinates.

60. The result of the last Article may also be obtained thus. Suppose that the given forces have a single resultant acting at the point $\left(x^{\prime}, y^{\prime}\right)$, and equivalent to the components $X^{\prime}$ and $Y^{\prime}$ parallel to the co-ordinate axes. It follows that the given forces will, with $-X^{\prime},-Y^{\prime}$ acting at the point $\left(x^{\prime}, y^{\prime}\right)$, form a system in equilibrium. Hence, by Art. 57,

$$
\Sigma X-X^{\prime}=0, \quad \Sigma Y-Y^{\prime}=0, \quad G-Y^{\prime} x^{\prime}+X^{\prime} y^{\prime}=0 .
$$

Of these three equations the first determines $X^{\prime}$, the second $Y^{\prime}$, and the third assigns a relation between $x^{\prime}$ and $y^{\prime}$, which is in fact the equation to the line in which the single resultant acts and at any point of which it may be supposed to act. If $\Sigma X$ and $\Sigma Y$ both vanish, it is impossible to find values of $x^{\prime}$ and $y^{\prime}$ that satisfy the last equation of the three, so long as $G$ does not vanish; this shews that if the forces reduce to a couple, it is impossible to find a single force equivalent to them.

61. In Art. 56, we have for the moment of the force $P_{1}$ about the origin the expression

$$
P_{1}\left(x_{1} \sin \alpha_{1}-y_{1} \cos \alpha_{1}\right),
$$

and this we may express by

$$
Y_{1} x_{1}-X_{1} y_{1} .
$$

Since $X_{1}$ and $Y_{1}$ are the rectangular components of $P_{1}$, we see by comparing the two expressions that the moment of a force about any origin is equal to the algebraical sum of the moments of its rectangular components about the same origin. (See Art. 54.) There are many such theorems connected with moments, and the demonstration of some of them 
is facilitated by otserving that accorling to the deferltion of a moment, it may be soometrically represented by twice the area of the triangle haning for its lase the straicht line which represents the force and for its sertex the pentas about which moments are taken. For example, we tway prove the theorem which we have already dedoced.

62. The a'gobratioal sum of the moments of thes compronemt jorces arich respoct bo any point in the plane cintuiving the soro firces is equal to the moment of the resultant of the tero firces.

I.et $A B, A C$ repreant two cotuponent forcen; complate the paraliclogram and draw the diagenal $A D$ representing the resultant force.

(1) Let 0 , the point about which the moments are to be taken, fall withome the angle $B . A C$ and that which is vertically opprite to it. Juin

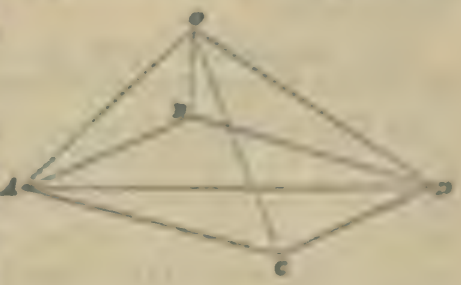
$O A, O B, O C, O D$.

The triangle $0 . A C$ haring for its base $A C$ and for its height the perpendicular from $O$ on $A O$ is equiralent is a triangle having $A C$ for its base and for its height the perpendicular from $B$ on $A C$, together with a triangle haring $B D$ for its base and for its height the perpendienlar from $O$ on $I B D$. This is obvious since $B D$ is equal and parallel to $A C$, and the perpendicular from $O$ on $A O$ is equal to the perpenilientas from $O$ on $B D$ together with the perpendicular from $B$ on $A C$. Hence we have

$$
\triangle A O C=\triangle B O D+\triangle A C D \text {. }
$$

Hence, adding the triangle $A O B$, we havo

$$
\triangle A O C+\triangle A O B=\triangle B O D+\triangle A B D+\triangle . A O B=\triangle . A O D ;
$$

that is, the moment of $A C+$ the moment of $A B=$ the moment of $A D$. 
(2) Let $O$ fall within the angle $B A C$ or its vertically opposite angle.

$$
\begin{aligned}
\triangle A O C & =\triangle A B D-\triangle B O D \\
& =\triangle A O B+\triangle A O D .
\end{aligned}
$$

Therefore

$$
\triangle A O D=\triangle A O C-\triangle A O B ;
$$

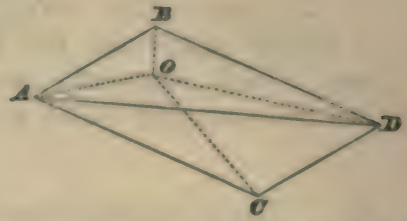

that is, the moment of $A D=$ the moment of $A C$-the moment of $A B$. $A \mathrm{~s}$ the moments of $A C$ and $A B$ about $O$ are now of opposile characters, the moment of the resultant is still equal to the algebraical sum of the moments of the components.

The proposition may also be readily shewn in the case where the two component forces are parallel; see Art. 37 .

In this example, however, nothing is gained in brevity or simplicity by the aid of Geometry; for the required result is an immediate consequence of the mechanical principle that the resolved part of the resultant along a straight line through $A$ at right angles to $A O$ is equal to the algebraical sum of the resolved parts of the components in the same direction.

63. Forces are represented in magnitude and position by the sides of a plane polygon taken in order; required the resultant.

Let the sides of the figure $A B C D E F$ represent the forces in magnitude and position; the first force being supposed to

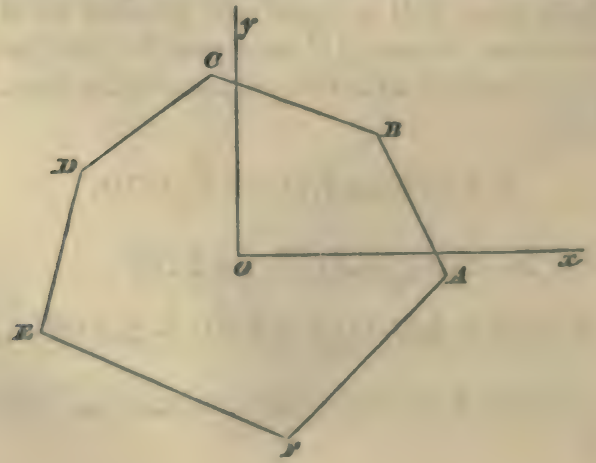


nct in the straight line $A B$ from $A$ towarde $B$, then enceses in the straight line $B O$ from $B$ towards $C$ and an wo.

As in Art. 36, the forees may be reglaced by a resslians force at an arbitrary origin $O$ and a coruples. The farmes is composed of all the forces $A B, D B, \ldots \ldots$ mosed earh parallel to itsclf up to $O$; the resaltant conseguently vasialies by Art. 21.

The moment of the resultant couple in the sum of the moments of the component cotples, asd is thereform reyre: senteal by twice the triangle $A O B+$ twiee the triangh $B O C$ $+\ldots$; thiat is, by tacice the arra of the plyjen. Hence the fories redace to a resultant couple measured $\mathrm{hy}$ iw ice the area of the polygon.

We may observe that the algebraical sum of the momesta of the two furons which form a couple is the neme about whatever point it be taten; it is in tact equal to the moment of the couple.

64. If the sum of the moments of the foress $P_{3}, P_{n}, P_{2} \ldots$ be required about a point whose co-ordinates are $k, k$ isolead of about the origin, we must in the expression for $Q$, in Art. 36, put $x_{1}-h, x_{1}-h, \ldots$ for $x_{1}, x_{2} \ldots$ respectirily, and $y_{1}-k, y_{0}-k, \ldots$ for $y_{1}, y_{2}, \ldots$ respoctively. Hence, denoting the result by $G_{1}$, we have

$$
\begin{aligned}
G_{1} & =\Sigma[Y(x-h)-X(y-h)\} \\
& =k \Sigma X-h \Sigma Y+\Sigma(Y x-X y) \\
& =k \Sigma X-h \Sigma Y+G .
\end{aligned}
$$

Hence the value of $G_{1}$ depends in general on the simation of the point about which we take moments. 18, however,

$$
l \mathbb{E} X-k \mathbb{Y} Y=\mathrm{a} \text { constant, }
$$

that is, if the point $(h, k)$ move along any straight lime parallel to the cirection of the resultant force ${ }_{h}$, iluen $G_{\text {, }}$ remains unchanged. 
If three different points exist with respect to which the sum of the moments vanishes, we have three equations

$$
\begin{aligned}
& k_{1} \Sigma X-h_{1} \Sigma Y+G=0, \\
& k_{2} \Sigma X-h_{2} \Sigma Y+G=0, \\
& k_{2} \Sigma X-h_{3} \Sigma Y+G=0 .
\end{aligned}
$$

Hence we deduce

$$
\begin{aligned}
& \left(k_{1}-k_{s}\right) \Sigma X=\left(h_{1}-h_{4}\right) \Sigma Y, \\
& \left(k_{2}-k_{2}\right) \Sigma X=\left(h_{8}-h_{3}\right) \Sigma Y .
\end{aligned}
$$

Unless the point $\left(h_{1}, k_{1}\right)$, the point $\left(h_{2}, k_{2}\right)$, and the point $\left(h_{3}, l_{3}\right)$ lie in a straight line, it is impossible that

$$
\frac{k_{1}-k_{2}}{h_{1}-h_{2}}=\frac{l_{2}-k_{3}}{h_{3}-h_{3}}
$$

we must therefore have

$$
\Sigma X=0, \quad \Sigma Y=0, \quad G=0 .
$$

Hence if the sum of the moments of a system of forces in one. plane vanish with respect to three points in the plane not in a straight line, that system is in equilibrium.

When a system of forces in one plane can be reduced to a single resultant, we have found in $\Lambda$ rt. 59 that the equation to the direction of the resultant is

$$
x^{\prime} \Sigma Y-y^{\prime} \Sigma X=\Sigma\left(I^{2} x-X y\right) \text {. }
$$

This may be written

$$
\Sigma\left\{Y\left(x^{\prime}-x\right)-X\left(y^{\prime}-y\right)\right\}=0 .
$$

The equation to the direction of the resultant thus in fact determines the locus of the points for which the algebraical sum of the moments of the forces is zero.

65. Hitherto we have supposed our axes rectangular. If they are oblique and inclined at an angle $\omega$, we may shew, as in Art. 56, that a system of forces in one plane may be reduced to $\Sigma X$ along the axis of $x, \Sigma Y$ along the axis of $y$, and a couple the moment of which is $\sin \omega \Sigma(Y x-X y)$. The latter part will be easily obtained, since the moment of the 
force $P_{4}$ is equivalent to the algeimical sum of the mononts of its components $X_{3}$ and $\Gamma_{i}$; and the perpendiectior we the former from the origin is $y_{3}$ sin $\omega$, and on the latter on, nin $\theta$.

The conditions for equilibrium are, an before,

$$
\Sigma X=0, \quad \Sigma Y=0, \quad \Sigma\left(\Gamma_{x}-X_{y}\right)=0
$$

The following Kxnmples may be solved by means of the principles given in the preceling Articles. When difenent rigid bodies oocur in a question, the ejuations of Ans 58 must hold with respect to eaik, in erder that there may le equilitirium. In cases where only three forees aet en a boly. it is often convenient to uhe the propesition of Art, As. S.ters by Art. 57 the moments of the foroes with respect tie eny origin must vanish, we may, if we phease, take different artgis and form the corresponding equation for each. She Art. 6 .

In some of the Examples we anticipate the resule of the subseyuent Chapters so lar as to saseme that the weiptet $f$ any boly acts through a definite and known poist, wCsh is the eentre of gravity of the body. When two be-lies are in contact it is assumed that whatever force ene exerts on the other the latter exerts an equal and ofpweite force on the former; if the bodies are smooth this foroe acts in the dimetion of the common normal to the surfaces at the print of contart. We restrict ourselves to the supposition of smowh baties until Chapter $\boldsymbol{x}$.

In attempting to solve the problems the student will find it advisable when the system invelses more than ane hedy to confine his attention to one at a rime of those boties which are capable of mótion, and to bo careful to tako into consideration all the foroes which act on that body. When bodies are in contact some letter should be used is desopia the mutual force between them, and the maproiteode of this force must be found from the equations of egullibrium of the bouly or bodieg which are capalle of motant. And when two of the bolies are connectud by a itring a leates nhenlly be used to denote the tension of the string, and the magainde of the tension must be found from the conditions of equi- 
librium of the body or bodies which are capable of motion. Beginners often fall into error by assuming incorrect values for the tensions of strings and the mutual forces between bodies in contact, instead of determining the correct values from the equations of equilibrium.

We will give here two propositions, respecting forces acting in a plane, which involve important results.

I. Forces act at the middle points of the sides of a rigid polygon in the plane of the polygon; the forces act at right angles to the sides, and are respectively proportional to the sides in magnitude: shew that the forces will be in equilibrium if they all act inwards or all act outwards.

The result here enunciated has been already shewn to be true in the case of a triangle; see the Proposition IV. at the end of Chapter II.; the general proposition is obtained by an inductive method.

Suppose for example that the proposition were known to be

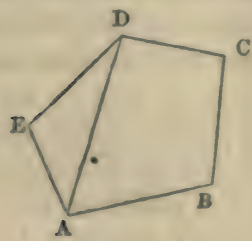
true for a four-sided figure also; then we can shew that it must be true for a five-sided figure. Let $A B C D E$ be a five-sided figure; and let forces act at the middle points of the sides in the plane of the figure, at right angles to the sides and respectively proportional to the sides in magnitude: suppose for the sake of distinctness that the forces all act outwards.

Join $A D$. By hypothesis a certain system of forces acting outwards on the four-sided figure $A B C D$ would be in equilibrium; and from this it follows that the assigned forces acting on $A B, B C, C D$ must be equivalent to a single force acting at the middle point of $A D$, towards the inside of the foursided figure $\triangle B C D$, proportional to $A D$ in magnitude.

Also the assigned forces acting on $D E, E A$ must in like manner be equivalent to a single force acting at the middle joint of $A D$, towards the inside of the triangle $A E D$, proportional to $A D$ in magnitude. 
Hence the two single forees balance ench other; and the system is in equilibrium.

In this mantur, knowing that the reate is tron for a tekangle, we can shew in succession that it is true for a figser of bur, five, sis, ... sides.

II. IRigh rods without weight are joined together by smooth hinges at their extremities, so is is form a plane polygon. Porces act at the midale points of the sibes ef the polyfon in the plane of the polygon; the fores act at zldte

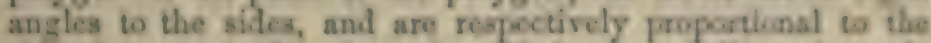
sides in magnitude; shew that, it the fores all act towanda or all act outwarls, where there is equilihriam, a circle can be described roumal the polygon.

Iet ABCDKF represent the polygon. Cunsiles one of the rods as AIR. This rot is arcal on by a firee at the mititle

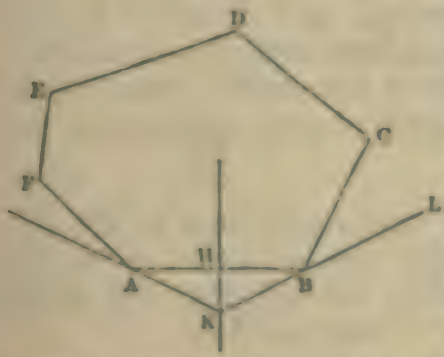
point $\|$ at right angles $60 . A B$. and by artions foon the hiscis at $\boldsymbol{A}$ and B. The former force is proportional to $A B$, and may he denoted by M.AB. The three forces must mert at a point, suppose $K_{\text {; then }}$ by $r 0$. solving parallel to $A B$, wi fisd that the actions at $A$ and $B$ mast be oqual; we will denote them by $R$. IResolve the forces at right angles to $A$ Li: tius

$$
A A B=2 R \text { sin } A B K \text {. }
$$

The action at $B$ on the rod $B C$ is equal and opposite to that on the rod $B A$; hence we obtain in the same manner

$$
\mu B C=2 R \sin C B L \text {. }
$$

Therefore $\quad \frac{\sin A B K}{\sin C I B L}=\frac{A B}{B C}=\frac{\sin A C B}{\sin C \cdot A B}$.

This shews that $K B L$ touches at $B$ the circle descriled round $A B C$.

Similarly $A K$ touclses at $A$ the circle descrited subsd $B .1 F$. 
But $A K$ and $B K$ are equally inclined to $A B$. Hence the two circles must coincide; that is, the points $F, A, B, C$ lie on the circumference of a circle.

In this way we shew that any four consecutive angular points of the polygon lie on the circumference of a circle; and hence it follows that all the angular points must lie on the circumference of the same circle.

It will be seen from the preceding results that the action at every hinge is the same, and is denoted by the product of $\mu$ into the radius of the circle described round the polygon.

\section{EXAMPLES.}

1. $A B C D$ is a quadrilateral and is acted on by forces which are represented in magnitude and direction by $A B$, $A D, C B, C D$; shew that the resultant coincides in direction with the straight line which joins the middle points of the diagonals $A C, B D$, and is represented in magnitude by four times this straight line.

2. Forces whose intensities are proportional to the sides of an isosceles triangle act along the sides of the triangle, those acting along the equal sides tending from the rertex; find the magnitude and position of their resultant.

Result. The required resultant is represented by a straight line which passes through the middle point of the base of the triangle, is parallel to one of the sides, and double that side in length.

3. The upper end of a uniform heavy rod rests against a smooth vertical wall; one end of a string is fastened to the lower end of the rod and the other end of the string is fastened to the wall; the position of the rod being given, find the point of the wall to which the string must be fastened, in order that the rod may be in equilibrium.

4. A uniform heavy rod is placed across a smonth horizontal rail, and rests with one end against a smooth vertical 
wall, the distance of which from the rail is $\frac{1}{16}$ th of the leapts of the rod; find the position of equilibrium.

Result. The rod makes an angle of $60^{\circ}$ with the hurizin.

5. $A B O$ is a triangular lamina; $A D, B E, C F$ ape the perpendiculars on the sides, and forces represented by the straight lines $B D, C D, C E, A E, A F, B F$ are applied is its lamina; shew that their resultant will pass through the centre of the circle described about the triangle.

6. $A B, A C$ are two ejual beams connected by a hisme at $A$, and by a string joining the extremities $B$ and $C: A B$ is fixed vertically, and a sphere of given weight and swiss is supported between the two beams: find tho pressure of the gyluere on each beam, and the tension of the kering.

7. An elliptic lamina is ncted on at the extremities of pairs of conjugate dinmeters by forens in its own plase toling outwands, and normal to its edge: shew that there will be equilibrium if the foree at the end of every diameter be proportional to the conjugate diameter.

8. A heary sphere hangs from a peg by a strias wioes length is equal to the radius, and it rests againat asootlar peg vertically below the former, the distance betwoen the two being equal to the diameter. Find the tension of the string and the pressure on the lower peg.

Rrsults. The tension is equal to the wright of the kqihere and the pressure to half the weight of the sphere.

9. Two equal rods without weight are connected at theis middle points by a pin which allows free motion in a vertival plane; they stand upon a horizmatal plane, am! their b/ges extremitics are connected by a thread which carries a witel. Shew that the wcight will rest half way botween the fora and the horizontal line joining the upper enils of the rots.

10. Two equal circular dises with smoweh elges. Jlawol on their flat sides in the corner between twe smoseli verisai planes inclined at a given angle, wuch each other in Be straight line bisecting the angle. Find the racius of the less: dise which may be pressed lefween them witious cassiag them to separatc. 
11. $\Lambda$ flat semicircular board with its plane vertical and curved edge upwards rests on a smonth horizontal plane, and is pressed at two given points of its circumference by two beams which slide in smooth vertical tubes; find the ratio of the weights of the beams that the board may be in equilibrium.

12. Two smooth cylinders of equal radii just fit in between two parallel vertical walls, and rest on a smooth horizontal plane without pressing against the walls; if a third cylinder be placed on the top of them, find the resulting pressure against either wall.

13. A smooth circular ring rests on two pegs not in the same horizontal plane; find the pressure on each peg.

14. Two spheres are supported by strings attached to a given point, and rest against one another; find the tensions of the strings.

15. Two equal smooth spheres, connected by a string, are laid upon the surface of a cylinder, the string being so short as not to touch the cylinder; determine the position of rest and the tension of the string.

16. A heavy regular polygon is attached to a smooth vertical wall by a string which is fastened to the middle point of one of its sides; the plane of the polygon is vertical and perpendicular to the wall, and one of the extremities of the side to which the string is attached rests against the wall; shew that whatever be the length of the string when the polygon is in equilibrium, the tension of the string and the pressure on the wall are constant.

17. A straight rod without weight is placed between two pegs, and forces $P$ and $Q$ act at its extremitics in parallel directions inclined to the rod; required the conditions under which the rod will be at rest and the pressures on the pegs.

18. Forces $P, Q, R, S$ act along the sides of a rectangle; find the direction of the resultant force.

19. Two weights $P, P$ are attached to the ends of two strings which pass over the same smooth peg and have their other extremities attached to the cnds of a beam $A B$, the 
weight of which is $\mathrm{W}$; shew that the inclinatiun of the beos to the herizon $=\tan ^{-a}\left(\frac{a-b}{a+b} \tan a\right) ; a, b$ heing the dlater.s of the centre of gravity of the beam from its enls, and $\sin \alpha=\frac{W}{2 l}$.

20. A square is placed with its plane vertical betwern two small pegs which are in the same horizontal lises shew that it will bo in eruilforium when the inclination of ene of its edges to the horizon $=\frac{f}{d} \sin ^{-1} \frac{a^{3}-c^{3}}{e}, 2 a$ Liag the length of a side of the styuare, and e the distasace betsrea the pres. Shew that the equilihrium will not be aff cterd by the aiplication of any firee wheh bisests the stridat lise joining the pegs and passes through the lowert priat of the Bquare.

21. One end of a string is fixod to the extremity of a smooth uniform rod, and the other to a ring witlont wrilyt which passes over the rod, and the string is hrmg ove a smooth per. Determine the lenst lengeh of the string foe which equilibrium is possible, and shew that the inclinatios of the rod to the vertical cannot be less than $45^{\circ}$.

22. A string 9 foet long has one end attaci-l of the cxfremity of a smooth unifurm heavy rod two foct is leserth. and at the other end carries a ring without weight whieh takles on the rou. The rod is suspended by means of the stouse fron a smooth peg; shes that if $\theta$ be the angle which the rod makes with the horizen, then

$$
\tan \theta=3^{-t}-a^{-t} \text {. }
$$

23. A square resis with its plane perpecticular bo a smooth wall, one ourner being attached to a point in the mall by a string whose length is equal to a bide of the kyours: shew that the distanoes of three of its anguiar points fres the wall are as 1,3 , and 4 .

21. One end of a beam, whes weight is IV, is flawe? on a smooth horizontal plane; the ocher enl, to whins w seic: is fastened, rests against anveluer smócth plane inction at an: angle a to the horizon; the string jusing ovis a jally at 
the top of the inclined plane hangs vertically, supporting a weight $P$. Shew that the beam will rest in all positions if a certain relation hold between $P, W$, and $\alpha$.

25. If a weight be suspended from one extremity of a rod moveable about the other extremity $A$, which remains fixed, and a string of given length be attached to any point $B$ in the rod, and also to a fixed point $C$ above $A$, and in the same vertical line with it, then the tension of the string varies inversely as the distance $A B$.

26. One end of a uniform beam is placed on the ground against a fixed obstacle, and to the other end is attached a string which runs in a horizontal direction to a fixed point in the same vertical line as the obstacle, and passing freely over it, is kept in tension by a weight $W$ suspended at its extremity, the beam being thus held at rest at an inclination of $45^{\circ}$ to the horizon. Shew that if the string were attached to the centre instead of to the end of the beam, and passed over the same fixed point, a weight $=\sqrt{ } 2 \mathrm{~W}$ would keep the beam in the same position.

27. Two equal beams $A B, A C$ connected by a hinge at $A$ are placed in a vertical plane with their extremities $B, C$ resting on a horizontal plane; they are kept from falling by strings connecting $B$ and $C$ with the middle points of the opposite sides; shew that the ratio of the tension of each string to the weight of each beam

$$
=\frac{1}{8} \sqrt{ }\left(8 \cot ^{2} \theta+\operatorname{cosec}^{3} \theta\right) \text {, }
$$

$\theta$ being the inclination of each beam to the horizon.

28. One end of a string is attached to a beam at the point $B$, and the other end is fastened to the lighest point $A$ of a fixed sphere of radius $r$. If the points of contact of the beam and string trisect the quadrant $A C$, shew that the distance between $B$ and the centre of gravity of the beam must be $2 r(2-\sqrt{ } 3)$.

29. A heavy rod can turn freely about a fixed hinge at one extremity, and it carries a heavy ring which is attached to a fixed point in the same horizontal plane with the hinge by means of a string of length equal to the distance between the point and the hinge. Find the position in which the rod will rest. 
30. Two equal heavy beams of solticient lenget, and connected by a hinge, are aupported by two smoss pers ia the same horizontal line; a spliere is placed Intwers thess. determine the position of equilibrium.

31. Forces $P, Q, R$ act along the sides $B C, C A, A B$ of a triangle, and their resultant pasacs through the entres of tie inseribed and circumseribed circles; shew that

$P: Q: R:: \cos B-\cos C: \cos C-\cos A: \cos A-\cos R$.

32. Find the position of equilibrium of a uniform beata resting in a vertical plane with one end prosing aysinst a vertical wall, and the other end supported by die eveves are of a vertical parabola whose vertex is at the fios of the wall and axis horizontal.

33. A uniform beam $P Q$ of giren weight and Irngth rosto in contact with a fised vertieal eirele wbose vertieal diameter is $A B$, in sueb a manter that strines $A P, I Q$ attachod to the rod and circle are tangents to the circle at the points $A$ asd $B$. Find the tensions of the strings, and shew that the cesslitioss of the problem require that the inclination of the bean to the vertical must be less than $\sin ^{-1} \frac{\sqrt{5}-1}{2}$.

34. Shew that no uniform rod can rest partly within and partly without a fixed smooth hemispherical bowl at an inothnation to the horizon greater than $\sin ^{-1} \frac{1}{\sqrt{3}}$.

35. The sides of a rigid plane polygon are acted on by forces at right angles to the sides and proportional to them in magnitude, all the forces acting in the plane of the polrgots, and being inwards; also the shles taken in the same eftes are severally divided by the points of applieation in the constant ratio of $p$ to $q$; shew that the system of forves is exgulvalent to a couple whose moment is

$$
\frac{\mu(p-q)}{2(p+q)} \geq
$$

where pa represents the force applied to any side of the polygon. 


\section{(64)}

\section{CHAPTER V.}

FORCES IN DIFFERENT PLANES.

66. To find the magnitude and direction of the resultant of any number of purallel forces acting on a rigid body, and to determine the centre of parallel forces.

Let the points of application of the forces be referred to a system of rectangular co-ordinate axes. Let $m_{1}, m_{2}, \ldots$ be the

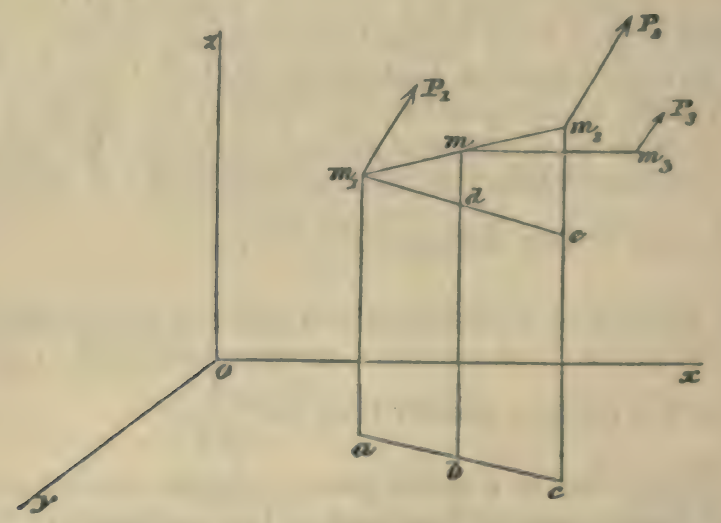

points of application; let $x_{1}, y_{1}, z_{1}$, be the co-ordinates of the first point, $x_{2}, y_{2}, z_{8}$ those of the second, and so on; let $P_{1}, P_{2}, \ldots$ be the forces acting at these points, those being reckoned positive which act in the direction of $P_{1}$, and those negative which act in the opposite direction.

Join $m_{1} m_{2}$; and talse the point $m$ on $m_{1} m_{2}$ such that

$$
m_{1} m=\frac{P_{3}}{P_{1}+P_{2}} \cdot m_{1} m_{g} ;
$$


then the resultant of $P_{1}$ and $P_{0}$ is $P_{1}+P_{0}$ and it acts through m parallel to $P_{1}$. (Ari, 37.)

Draw $m, a, m b, m_{0}$ p perpendicular to the plane of $(x, y)$. meeting that plane at $a, b, c$; draw m, ile parallel to ale moeting $m b$ at $d$ and $m_{g} 0$ at $e$. Then, by nimilar triasgies,

$$
\frac{m_{1} m}{m_{1} m_{i}}=\frac{m d}{m_{i} n}=\frac{m b-\varepsilon_{i}}{\varepsilon_{2}-\varepsilon_{1}},
$$

therefore

$$
m b-z_{1}=\frac{P_{1}}{P_{1}+T_{2}}\left(\varepsilon_{1}-z_{1}\right) \text {; }
$$

therefore

$$
m b=\frac{P_{2}+P_{2} e_{1}}{P_{1}+P_{2}} \text {. }
$$

This gives the ordinate parallel to the axis of a of the point of application of the resultant of $P_{1}$ and $P_{0}$.

Then supposing $P_{1}$ and $P_{2}$ to he replaced by $P_{1}+P_{0}$ arting at $m$, the resultant of $P_{1}+P_{3}$, and $P_{3}$ is $P_{3}+P_{4}+P_{6}$, and the ordinate of its point of application

$$
=\frac{\left(P_{1}+P_{3}\right) m b+P_{2}}{P_{1}+P_{0}+P_{3}}=\frac{P_{2}+P_{2} P_{2}+P_{2}}{P_{1}+P_{0}+P_{0}} ;
$$

and this process may be extendod to any number of parallal forees. Let $R$ denote the resultant force and o the ordinate of its point of application; then

$$
R=\Sigma P_{0}=\frac{\Sigma}{\Sigma} P_{z}
$$

Similarly, if $\bar{x}, \bar{y}$ be the other co-urdinates of the point of application of the resultant,

$$
\bar{x}=\frac{\Sigma P_{x}}{\Sigma T} ; \quad \bar{y}=\frac{\Sigma P_{y}}{\Sigma F} .
$$

The values of $\bar{x}, \bar{y}, \bar{z}$ are indeprentent of the anglise which the directions of the forces make with the axes. Henee if these directions be turned alout the joints of application of the forces, "their parnllelisu being preserved, the joint of T. \&. 
application of the resultant will not move. For this reason this point is called the centre of the parallel forces.

67. The moment of a force with respect to a plane is the product of the force into the perpendicular distance of its point of application from the plane.

In consequence of this definition, the equations for determining the position of the centre of parallel forces shew that the sum of the moments of any number of parallel forces with respect to any plane is equal to the moment of their resultant.

68. If the parallel forces all act in the same direction the expression $\Sigma P$ cannot vanish; hence the values of the coordinates of the centre of parallel forces found in Art. 66 cannot become infinite or indeterminate, and we are certain that the centre exists. But if some of the forces are positive and some negative, $\Sigma P$ may vanish, and the results of Art. 66 become nugatory. In this case, since the sum of the positive forces is equal to the sum of the negative forces, the resultant of the former will be equal to the resultant of the latter. Hence the resultant of the whole system of forces is a couple, unless the resultant of the positive forces should happen to lie in the same straight line as the resultant of the negative forces.

We shall give another method of reducing a system of parallel forces.

69. To find the resultant of a system of parallel forces acting upon a rigid body.

Let $P_{1}, P_{2}, \ldots$ denote the forces. Take the axis of $z$ parallel to the forces. Let the plane of $(x, y)$ meet the direction of $P_{1}$ at $M Y_{1}$, and suppose $x_{1}, y_{1}$ the co-ordinates of this point.

Draw $M_{1} N_{i}$ perpendicular to the axis of $x$ meeting it at $N_{i}$. At the origin $O$, and also at $N_{1}$, apply two forces each equal and parallel to $P$, and in opposite directions. Hence the force $P_{1}$ at $M_{1}$ is equivalent to the following system,

(1) $P_{1}$ at $O$;

(2) a couple formed of $P_{1}$ at $M_{1}$ and $P_{1}$ at $N_{1}$;

(3) a couple formed of $P_{1}$ at $N_{1}$ and $P_{1}$ at $O$ : 


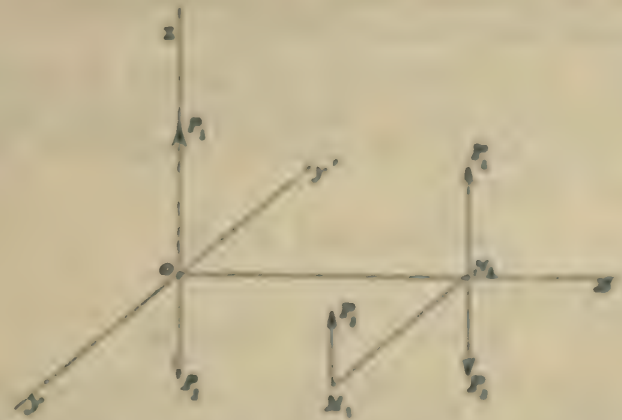

The moment of the fint onuple in $P_{3} y_{1}$, and this ocupiles. without altering its effict, may ho trasusterred to the plane of $(y, z)$, which is parallel to its original plane. The monest of the second couple is $I_{1} x_{1}$, and the couple is in the plane of $(x, z)$.

If we effect a similar transformation of all the firme, wo have, as the resultant of the system the fullowing gystem,
(1) a force $\Sigma P$ acting at $O$;
(2) a couple $\Sigma P_{y}$ in the plane of $(y, z)$;
(3) a couple $\mathbb{S} P x$ in the plane of $(x, 2)$.

The first couple tends to turn the body from the axis of $g$ to that of $\mathrm{z}$, and the socond from the axis of $\mathrm{x}$ to that of $\mathrm{z}$. We may therefore take $O x$ as the axis of the fint couple acoonling to the definition in Art. 41. For the second cutple, bowever, we must either take $O_{y}$ as the axis, or comider it as a couple turning from a to $x$, of which the moment is - SPe and tin axis Oy. Adopting the latter method, we may replace the two couples by a single couple of which the moment is $G$, where

$$
G^{2}=(\mathbb{S} P x)^{2}+\left(\mathbb{S} P_{y}\right)^{2},
$$

and the axis is inclined to the axis of $x$ at an angle a given by the equations

$$
\cos a=\frac{\Sigma P_{y}}{G} ; \quad \sin a=\frac{-\Sigma P_{x}}{G} .
$$


70. To find the conditions of equilibrium of a system of parallel forces acting on a rigid body

$\Lambda$ system of parallel forces can always be reduced to a single force and a couple. Since these cannot balance, and neither of them singly can maintain equilibrium, they must both vanish. That is,

$$
\Sigma P=0 \text {, and } G=0 ;
$$

\section{the latter requires that}

$$
\Sigma P x=0 \text {, and } \Sigma P y=0 .
$$

Hence $a$ system of parallel forces acting on a rigid body will be in equitibrium if the sum of the forces vanishes, and also the sum of the moments vanishes with respect to two planes at right angles to each other and parallel to the forces.

Conversely, if the forces are in equilibrium the sum of the forces will vanish, and also the sum of the moments with respect to any two planes at right angles to each other and parallel to the forces.

71. When $\Sigma P=0$, the forces reduce to a couple of which the moment is $G$. When $\Sigma P$ is not $=0$, the forces can always be reduced to a single force; this has already appeared in Art. 66, and may also be shewn thus. The forces will reduce to a resultant $R$ acting at the point $\left(x^{\prime}, y^{\prime}\right)$, parallel to the original forces, provided a force $-R$ acting at this point will with the given forces maintain equilibrium. The necessary and sufficient conditions for this are, by $\mathrm{Art}$. 70,

Hence

$$
\Sigma P-R=0, \quad \Sigma P x-R x^{\prime}=0, \quad \Sigma P y-R y^{\prime}=0 .
$$

$$
R=\Sigma P, \quad x^{\prime}=\frac{\Sigma P x}{\Sigma P}, \quad y^{\prime}=\frac{\Sigma P y}{\Sigma P^{3}} \text {. }
$$

These results agree with those of Art. 66 .

72. To find the resultants of any number of forces acting on a rigid body in any directions.

Let the forces be referred to three rectangular axes $O x, O y$, $O z$; and suppose $P_{1}, P_{3}, P_{3}, \ldots$ the forces; let $x_{1}, y_{1}, z_{1}$ be the co-ordinates of the point of application of $P_{1}$; let $x_{2}, y_{2}, z_{\mathrm{g}}$ be the co-ordinates of the point of application of $Y_{g} ;$ and so on. 
Int $A_{1}$ be the point of application of $P_{1}$, reselre $P_{3}$ ises components $X_{1}, Y_{1}, Z_{1}$, parallel to the co-urdinate axra La

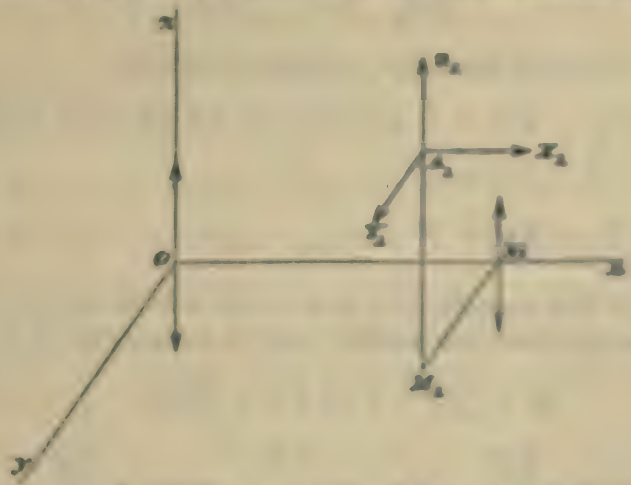

the direction of $Z_{1}$ meet the plane of $(x, y)$ at $M$, and dram $M_{s} X_{\text {, perpendicular to }} O_{x}$. $A_{p p l y}$ at $N_{i}$ and also at $Q$ iwe forces each equal and parallel to $\%$ and in opposite dirnctess. Hence $Z_{\text {at }} A_{1}$ or $\mathbb{I}_{\mathrm{f}}$ is equiralent to $Z_{2}$ at $Q$, and $\mathrm{tr}$ : couples, the former having its moment $=Z, N M_{1}$, and wikch may be supposed to act in the plane of $(y, 2)$, and the latier having its moment $=Z_{1} \cdot O X_{1}$ and acting in the plane of $(a, x)$.

We shall consider those couples as positive which tend to turn the body round the axis of $x$ from $y$ to $z$, also these which tend to turn the body round the axis of $y$ from a to as, and those which tend to turn the body round the asis of a from $x$ to $y$.

Hence $Z_{1}$ is replaced by $Z_{4}$ at $O$, a couple $Z_{Z_{3}}$ in the plase of $(y, z)$, and a couple $-Z, x_{1}$ in the plane of $[z, x)$. Simlarly $X_{1}$ may be replaced by $X_{\text {at }} 0$, a couple $X_{B}$, in the plane: of $\left(z, x_{i}\right)$ and a couple $-x_{y_{3}}$ in the plane of $\left\langle r, y_{j}\right.$. And $Y_{i}$ may be replaced by $Y_{\text {, at }} O$, a couple $Y$, in in the plase of $(x, y)$, and a couple $-Y_{2}$ in the plane of $(y, z)$. Therefine the foree $P_{1}$ may be replaced by $x_{1}, \gamma_{1}, Z_{\text {acting at }} D_{1}$ and the couples of which the moments are, by Art. 45?

$$
\begin{aligned}
& Z y_{1}-Y_{2}=\text { in the plane of }(g, z) \text {, } \\
& X_{1} z_{1}-Z_{1} x_{1} \ldots \ldots \ldots \ldots \ldots \ldots\left(z_{1} x\right), \\
& Y_{1} x_{1}-X_{2} y_{1} \ldots \ldots \ldots \ldots \ldots \ldots(x, y) \text {. }
\end{aligned}
$$


By a similar resolution of all the forces we shall have them replaced by the forces

\section{$\Sigma X, \quad \Sigma Y, \quad \Sigma Z$,}

acting at $O$ along the axes, and the couples

$$
\begin{aligned}
& \Sigma(Z y-Y z)=L \text { suppose, in the plane of }(y, z), \\
& \Sigma(X z-Z x)=M \ldots \ldots \ldots, \ldots \ldots \ldots \ldots \ldots(z, x), \\
& \Sigma(Y x-X y)=N \ldots \ldots \ldots, \ldots \ldots \ldots \ldots \ldots(x, y) .
\end{aligned}
$$

Let $R$ be the resultant of the forces which act at $O ; a, b, c$ the angles its direction makes with the axes; then, by Art. 24,

$$
\begin{gathered}
R^{2}=(\Sigma X)^{2}+(\Sigma Y)^{2}+(\Sigma Z)^{2}, \\
\cos a=\frac{\Sigma X}{R}, \quad \cos b=\frac{\Sigma Y}{R}, \quad \cos c=\frac{\Sigma Z}{R} .
\end{gathered}
$$

Let $G$ be the moment of the couple which is the resultant of the three couples $L, M, N ; \lambda, \mu, \nu$ the angles its axis makes with the co-ordinate axes; then, by Art. 50,

$$
\begin{gathered}
G^{2}=L^{2}+M^{2}+N^{2}, \\
\cos \lambda=\frac{L}{G}, \quad \cos \mu=\frac{M}{G}, \quad \cos \nu=\frac{N}{G} .
\end{gathered}
$$

The convention adopted in the present Article for distinguishing the signs of couples agrees with that in Art. 41 when the axes of $x, y$, and $z$ are drawn as in the present figure, but the conventions will not necessarily coincide if the figure be modified; for example, if the axes of $y$ and $z$ be retained as in the figure, but the positive part of the axis of $x$ directed to the left instead of the right, they will not coincide. The convention of the present Article is that which we shall hereafter always retain.

73. To find the conditions of equilitrium of any number of furces acting on a rigid body in any directions.

A system of forces acting on a rigid body can always be reduced to a single force and a couple. Since these can- 
nut halance each other and cannot meparately maineain eysi. librium they unst both vanish. Hence $I i=\hat{U}$, and $\sigma=0_{\text {; }}$ therefore and

$$
\begin{gathered}
(\mathbf{X} X)^{2}+(\mathbf{\Sigma})^{2}+(\mathbf{Z} Z)^{2}=0 \\
\boldsymbol{L}^{3}+\boldsymbol{M}^{3}+\boldsymbol{N}^{2}=0
\end{gathered}
$$

'These lead to the six conditions

$$
\begin{gathered}
\Sigma X=0, \quad \Sigma Y=0, \quad \leq X=0, \\
\Sigma\left(Z y-Y_{z}\right)=0, \quad \leq\left(X z-X_{x}\right)=0, \quad \Sigma\left(X z-X_{y}\right)=0 .
\end{gathered}
$$

74. A verbal enunciation may be given of the lase three equacious by means of a new definition. For the make af convenienoe, we repeat two definitions already given in Arta. 54 and 67.

Moment of a force with respect to a poine. The motuent of a force with respect to a point is the jroduct of the firme into the perpendicular from the point on the direcion of the force.

Moment of a force with respect to a plame. The mament of a force with respect to a plane is the product of the forve into the distance of its point of application from then plane.

Moment of a force with respect to a servight lins. Resolve the force into two components respectively parallel asud perpendicular to the straight line; the product of the compoment perpendienlar to the line into the shorest disauce between the straight line and the direction of this companent is called the momont of the force with respect to the straight line.

Hence the unoment of a furce with respect to a straight line is equal to the moment of the component of the fosce peryondieular to the straiglit line with respect to the puint as wheh a plane drawn through this component perpendieular to the straight line meets the straight line. Henes, by Art. 62, the moment of the force may be found by taking the $\mathrm{sum}$ of the monents of any two furces into which the perpendicular camponent may be resolved.

If the force is parallel to the given araight line, its momess abont the straight line is zemo. If the furce is pergenofingur to the given straight line, is mosment about the stragetit line is the product of the force into the shortest distance besween it and the given straight line. 
75. Suppose we require the moment of the foree $P$ about the axis of $z$; we resolve $P_{1}$ into the forees $Z_{1}$ parallel to the axis of $z$ and $Q_{1}$ perpendicular to the axis of $z$, where $Q_{1}$ is itself the resultant of $\boldsymbol{X}_{1}$ and $Y_{2}$. The moment of $Q_{1}$ with respect to the axis of $z$ is equal to the algebraical sum of the moments of its components $X_{1}$ and $Y_{1}$; that is, to $Y_{1} x_{1}-X_{1} y_{1}$. Hence $N$ in Art. 72 denotes the sum of the moments of the forces round the axis of $z$, and similar meanings arise for $L$ and $M$.

Hence, the forces acting on a rigid body will be in equilibrium if the sums of the resolved parts of the forces parallel to three straight lines at right angles to each other vanish, and the sums of the moments of the forces with respect to these straight lines also vanish.

Conversely, if the forces are in equilibrium, the sum of the resolved parts of the forces in any direction will vanish, and also the sum of the moments of the forces with respect to any straight line.

76. In order to interpret the meaning of $G$ we observe that if we keep to the same origin, the moment of this couple and the direction of its axis must be independent of the directions of the co-ordinate axes. For $R$, being the resultant of all the given forces, supposing them applied at a point, is of course independent of the directions of the axes. If by a new choice of axes we obtain $G^{\prime}$ as the resultant couple, then $R$ and $G$ must be equivalent to $R$ and $G^{\prime}$, and therefore $R, G,-R,-G^{\prime}$ must form a system in equilibrium. But this is impossible unless $G=G^{\prime}$ and the axes of $G$ and $G^{\prime}$ are coincident or parallel.

Since the direction of the co-ordinate axes is arbitrary, suppose the axis of $x$ to coincide with the axis of $G$; then $M=0$, $N=0$, and $L$ and $G$ are identical.

Hence $G$ is equal to the sum of the moments of the given forces with respect to the struight line which is the a.xis of $G$.

77. Suppose a force $P$ acting at the point $(x, y, z)$, and let $X, Y, Z$ be its components parallel to the axes. Then, by Art. 72, $P$ at the point $(x, y, z)$ is equivalent to $P$ at the origin, together with the couples $Z y-I^{\prime} z, X z-Z x, Y x-X y$ 


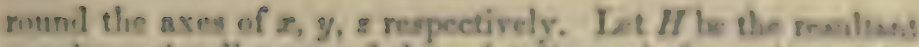
(muple, $r$ the disfance of the point its, $y, z$ ) from tha atifis, and $a$ the angle between $r$ and $P$; thrn

$$
\begin{aligned}
& I^{\prime}=\left(Z y-\Gamma_{z}\right)^{\circ}+\left(X_{z}-Z z_{x}\right)^{\circ}+\left(\Gamma_{z}-X_{y_{i}}{ }^{\prime}\right. \\
& =\left(x^{2}+y^{2}+z^{2}\right)\left(X^{2}+I^{2}+Z^{2}\right)-\left(x \boldsymbol{X}+g \Gamma^{2}+z\right)^{\prime} \\
& =r^{2} I^{n}-r^{2} I^{m}\left(\frac{x}{r} \frac{x}{P}+\frac{y}{r} \frac{Y}{P}+\frac{e Z}{r}\right)^{\prime} \\
& =r^{2} P^{\circ}\left(1-\cos ^{\circ} a\right) \text {, }
\end{aligned}
$$

therefore $\|=r P \sin a$

Thus, as we might have anticipatod, $I$ is the moment of the couple formed ly $P$ at the point $\{x, y, a$, and a firse at the origin eyual to $P$ and acting in a parallel and opposite dirretion. Ifence $G$ is the exuple formod by compounding the couples similar to $I I$ arising from all the forces of the systern.

78. As an example of Art.73 we may take the cose in which all the forees are purallet. Iat $\alpha, \beta, \gamma$ be the andes which the dircetion of the furces $P_{1}, P_{0} \ldots \ldots$ malis with the axes. Then the equations of equilitrium reduce tis

$$
\begin{gathered}
\Sigma P=0, \\
\Sigma P(y \cos \gamma-z \cos \beta)=0, \\
\Sigma P(z \cos \alpha-x \cos \gamma)=0, \\
\Sigma P(x \cos \beta-y \cos a)=0
\end{gathered}
$$

The lnst three equations may be written thus:

$$
\frac{\Sigma P_{x}}{\cos \alpha}=\frac{\Sigma P_{y}}{\cos \beta}=\frac{\Sigma P_{2}}{\cos \gamma} \text {. }
$$

Hence we can deduce the conditions that a systrm of paralled forces may maintain a body in equilibrium, hasereer if be turned abume the ir poines of applicastien. Fer the poocoling equatinus must then hold whaterer $2, \beta$, $\gamma$ may be Thas we must have

$$
\Sigma l^{\prime}=0, \quad \Sigma P_{x}=0, \quad \Sigma P_{y}=0, \quad \Sigma I=0 .
$$


79. In Art. 72 we have reduced the forces acting on a body to a force $R$ and a couple $G$. If $G$ vanish there remains a single force; and if $R$ vanish, a single couple. If neither $R$ nor $G$ vanish the forces may reduce to a single force; we proceed to shew when this is possible.

To find the condition among the forces that they may have a single resultant.

Any system of forces can be reduced to a single force $R$ and a couple $G$; if then the forces can be reduced to a single resultant $S$, it follows that $G, R$, and $-S$ are in equilibrium. If $R$ and $-S$ do not form a couple, they can be reduced to a couple $G^{\prime}$ and a force $R^{\prime}$; therefore $R^{\prime}$ must balance the couple compounded of $G$ and $G^{\prime}$. 'This is impossible by $\Lambda$ rt. 40. Hence $R$ and $-S$ must form a couple, and this couple must have its plane coincident with that of $G$, or parallel to that of $G$, in order that it may balance $G$. Therefore that the forces may have a single resultant, the direction of $R$ must be parallel to the plane of $G$, or coincident with it ; that is, must be at right angles to the axis of $G$. Hence, using the notation of Art. 72,

therefore

$$
\cos a \cos \lambda+\cos b \cos \mu+\cos c \cos \nu=0,
$$

$$
L \Sigma X+M \Sigma Y+N \Sigma Z=0 .
$$

80. Conversely, if $L \Sigma X+M \Sigma Y+N \Sigma Z=0$, and $\Sigma X, \Sigma Y$, $\Sigma Z$ do not all vanish, the forces can be reduced to a single force. For the plane of the couple $G$ may be made to contain the force $R$, and the couple may be supposed to have each of its forces $=R$ and its arm consequently $=\frac{G}{R}$; the couple may then be turned round in its own plane until the force at one end of its arm balances the resultant force $R$, and there remains $R$ at the other end of the arm.

81. When the forces are reducible to a single resultant, to find the equations to the straight line in which it acts.

Let $L, M, N$ denote the moments of the forces round the co-ordinate axes; $L^{\prime}, M^{\prime}, N^{\prime}$ the moments of the forces round 
axes parallel to the co-ordinnte axes drawa thrumgh the peetut

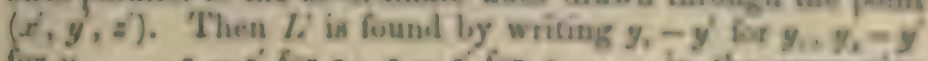
for $y_{1}, \ldots \ldots, z_{1}-z_{\text {for }} z_{1} z_{0}-\varepsilon$ for $z_{0}, \ldots \ldots$ in the expromatom $\mathbf{s}(z y-Y z)$. Therefore

Similarly

$$
\begin{aligned}
I & =\mathbf{\Sigma}\left[Z\left(y-y^{\prime}\right)-Y\left(z-z^{\prime}\right]\right) \\
& =L-y^{\prime} \mathbf{Z} Z+z^{\prime} \mathbf{I} Y .
\end{aligned}
$$

$$
\begin{aligned}
& W^{\prime}=\Sigma\left[X\left(z-z^{\prime}\right)-Z\left(x-x^{\prime}\right)\right] \\
& -\mathbb{M}-z^{\prime} \mathbf{Z} \boldsymbol{X}+x^{\prime} \mathbb{Z} \mathbb{Z}_{1} \\
& N^{\prime \prime}=\Sigma\left|Y\left(x-x^{\prime}\right)-X(y-y)\right| \\
& =\mathbf{V}-x^{\prime} \mathbf{X} Y+y^{\prime} \mathbf{I} X \text {. }
\end{aligned}
$$

If $x^{\prime}, y^{\prime}, z^{\prime}$ can he so taken $n a$ in make $L, M r$, and $N$ vanish, the forces reduce to a single resultant prosing thruagh the point $(x, y, 2)$. The three equations

$$
\begin{aligned}
& I .-y \mathbf{Z} Z+Z \mathbb{Z} Y=0 \ldots \ldots \ldots \ldots \ldots \ldots(1) \text {, }
\end{aligned}
$$

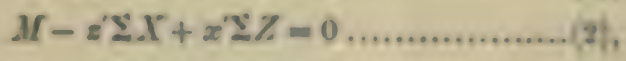

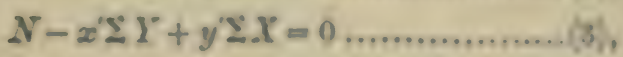

are equiralent to two independent equations; for if we eliminute $z$ from (1) and (2), we have

$$
L \mathbf{L} X+M \mathbf{I} Y+\mathbf{Z} Z\left(x^{\prime} \mathbf{I} Y-y^{\mathbf{Z} X}\right)=0 \text {. }
$$

But

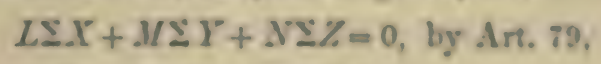

therefore

$$
\mathbf{V}-x^{\prime} \mathbf{Y} Y+y^{\prime} \mathbf{X}=0 .
$$

Thus (3) is a necessary conserquence of (1) and (2). Hence (1) and (2) will determine a straight line at enry point of which the resultant couple ranishes; that is, the straight line in which the single resultant force acts.

82. By the following method we may determine at otne the condition for the existence of a single resultant and the equations to its direction.

Suppose that the forces can be reluced to a single fom acting at the point $(x, y, z)$. Iet the single force le restred 
into components $X^{\prime}, Y^{\prime}, Z^{\prime}$ parallel to the co-ordinate axes; then if we add to the given system $-X^{\prime \prime},-Y^{\prime}$, and $-Z^{\prime}$, acting at the point $\left(x^{\prime}, y^{\prime}, z^{\prime}\right)$ parallel to the axcs respectively, there will be equilibrium. Hence, by $\Lambda \mathrm{rt} .73$,

$$
\begin{aligned}
& \Sigma X-X^{\prime \prime}=0, \quad \Sigma Y-Y^{\prime}=0, \quad \Sigma Z-Z^{\prime}=0 \ldots \ldots \ldots(1), \\
& L-Z^{\prime} y^{\prime}+Y^{\prime} z^{\prime}=0 \\
& M-\Gamma^{\prime} z^{\prime}+Z^{\prime} x^{\prime}=0 \\
& N-Y^{\prime} x^{\prime}+X^{\prime} y^{\prime}=0
\end{aligned}
$$

Equations (1) determine $X^{\prime}, Y^{\prime}, Z^{\prime}$. It might at first appear that equations (2) would determine $x^{\prime}, y^{\prime}, z^{\prime}$; but if we proceed to solve them, we find that they cannot be simultancously true unless

$$
L \Sigma X+M \Sigma Y+N \Sigma Z=0
$$

and if this condition be satisfied, and $\Sigma X, \Sigma Y, \Sigma Z$ do not all vanish, then any one of the equations may be derived from the other two, so that there are only two independent equations. Hence that the forces may have a single resultant the above condition must be satisfied, and then any two of equations (2) will determine the locus of points at which this single resultant may be supposed to act. From the form of equations (2) it is obvious that this locus is a straight line, and that its direction cosines are proportional to $X^{\prime}, Y^{\prime}, Z^{\prime}$, as might have been anticipated.

In order that the force which replaces the system may pass through the origin, we must have

$$
L=0, \quad M=0, \quad N=0 .
$$

83. Although a system of forces cannot always be reduced to a single resultant, it can always be reduced to teco forces. For we have shewn that the system may be replaced by a force $I R$ at the origin, and a couple $G$ lying in a plane through the origin; one of the forces of $G$ may be supposed to act at the origin, and may be compounded with $R$ so that this resultant and the other force of ( $\dot{r}$ are equivalent to the whole system. Since the origin is arbitrary, we sce that when a system of forces is not reducible to a single force it can be reduced to two forces, one of which can be made to pass through any assigned point. 
84. When three forres maintain a bealy in gevilizerien, they must lie in the sume plane.

Draw any straight line interneting the diswetions of tso of the forces and not parallel wo the thind fores, and tahe thas straight line for the axis of $x$. Then the first iwo borme laswe no moment round the asis of ar: therefore the ejuation $L=0$ rejuires that the third firce shenll have no moment romd the axis of $r$; that is, the direstion of the thind form mas pass through the axis of $a$. Since then any straight Fine, which meits the difections of two of the forres, atal is mo parallel to the direction of the third, meets that direction, the three fires must lie in one plater.

Combining this proposition with that in Art. is, we ace that if three forces kecp a bealy in cquilibrium, thery mast all lie in the same plane and must mect at a point or be parallel.

85. If the axes of co-ordinates be oblique, suppoes $\angle \mathrm{an}$, to dinote the sines of the angles between the ases of $y$ and $\mathrm{s}$, $z$ and $x, x$ and $y$, respectircly; then we may slerw, as in Art. 72 , that any system of forws can be reduced to $\mathbb{X}, \mathbf{X} Y$, X, acting at the origin along the axes of $x, y, z$ respectirnly, and three couples in the threo co-ordinate planes, harring ilais moments equal to $I_{2}, m . M, n N$ respoctircly, where, as beloxe,

$$
L=\Sigma(Z y-Y z), \quad M=\Sigma(X z-Z x), \quad N=\Sigma\left(X_{x}-X_{y}\right\rangle .
$$

Also for equilibrium, we must have, as before,

$$
\begin{aligned}
& \Sigma X=0, \sum Y=0, \quad \sum X=0 ; \\
& L=0, \quad M=0, \quad N=0 .
\end{aligned}
$$

That the forces may almit of a single resultant we must lase, as before,

$$
I \Sigma X+M I Y+N \Sigma Z=0,
$$

and $\mathbf{\Sigma} X, \mathbf{Y}, \mathbf{\Sigma} Z$ not all rasishing.

The following propositions are connected with the sulfest of the present Cliapter. 
I. Forces act at the angular points of a tetrahedron in directions respectively perpendicular to the opposite faces and proportional to the areas of the faces in magnitude: shew that the forces will be in equilibrium.

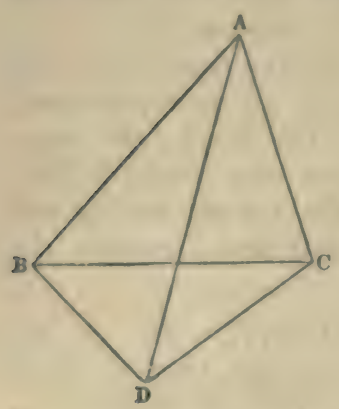
dron.

Let $A B C D$ represent the tetrahe-

(1) Resolve the forces parallel to $A B$. Let $p$ denote the perpendicular from $A$ on the face $B C D$; then the resolved part of the force at $A$ is $\frac{p}{A B} \times$ area of $B C D$, that is, $\frac{3 \text { volume of tetrahedron }}{A B}$.

We obtain the same expression for the resolved part of the force at $B$. The forces at $C$ and $D$ have no resolved part parallel to $A B$. Thus the forces resolved parallel to $A B$ vanish.

(2) Take moments round $A B$. Let $q$ denote the perpendicular from $C$ on the straight line $A B ; \theta$ the angle between the planes $B A D$ and $B A C$. Then the moment of the force at $C$ is $q \cos \theta$. area of $A B D$, that is,

$\frac{q A B \cos \theta \text {. area of } A B D}{A B}$ or $\frac{2 \cos \theta \text {. area of } A B C \text {. area of } A B D}{A B}$.

We obtain the same expression for the moment of the force at $D$. Thus the moments round $A B$ vanish.

Since these results hold for any edge of the tetrahedron the forces must be in equilibrium.

II. Four forces act on a tetrahedron at right angles to the faces and proportional to their areas, the points of application of the forces being the centres of the circles circumscribing the faces: shew that if the forces all act inwards or all act outwards they will be in equilibrium.

In this case the forces all pass through a point, namely the centre of the sphere described round the tetrahedron. 
Hence we only reupire the fint part of the inventigation in the preceding proposition to eatablish that the furces are in equilibrium.

Or we may resolve the forces at right angles to a fane instead of purallel to an edge, and thas obtain the reselt. For resolve the forees at right angles to the face $B C D$ : we have one force represented by the area $B C D$, and the resolved parts of the other forces are represented by the pirm. jections of the respective aress $B A C, C A D, D . A B$ on $B D$. And the sum of these projections is equal to BCD. Thus the forces resolved at right angles to $B C D$ ranish.

Similarly the forces resolved at right angles to any other face ranish.

III. By a process similar to that used in establishing the Proposition 1. at the end of Chapter Ir. we can extuna the preceding Proposition to the case of any polyhodron boundel by triangular faces. Thus we obtain the fallowing reult: Forces act on a polyhedron bounded by triangular faces at right angles to the faces and proportional to their areas, the points of application of the forces being the centres of twe circles circumscribing the faces; shew that if the forma all act inwards or all act outwards they will be in equilibriam.

IV. If four fores acting on a rigid boily are in muilibrium, and a tetrahedron be constructed by drawing flanes at right sngles to the directions of the furces, the furves will be respectively proportional to the areas of the faces.

This is the converse of II. and may be rearlily demonstrated: for by resolving the forces in any dircetion, and projecting the areas on a plane at right angles to that dirmotion, we find that the four forces are connected by the same linear relation as the four areas.

We infer from this result that the areas in the present theorem must be respectively proportional to the volume considered in the Proposition X. at the end of Chapter H: thus we indirectly arrive at a geometrical truth. 


\section{EXAMPLES.}

1. Four parallel fores act at the angles of a plane quadrilateral and are inversely proportional to the segments of its diagonals nearest to them; shew that the point of application of their resultant lies at the intersection of the diagonals.

2. Parallel forces act at the angles $A, B, C$ of a triangle and are respectively proportinnal to $a, b, c ;$ shew that their resultant acts at the centre of the inscribed circle.

3. A cone whose vertical angle is $30^{\circ}$, and whose weight is $W$, is placed with its vertex on a smooth horizontal plane; shew that it may be kept with its slant side in a vertical position by a couple whose arm is equal to the length of the slant side of the cone, and each force $\frac{3 W}{16}$.

4. Six equal forces act along the edges of a cube which do not meet a given diagonal, taken in order; find their resultant.

Result. A couple, the moment of which is $2 P a \sqrt{ } 3$, where $P$ denotes each force and $a$ the edge of the cube.

5. $\Lambda$ cube is acted on by four forces; one force is in a diagonal, and the others in edges no two of which are in the same plane and which do not meet the diagonal; find the condition that the forces may have a single resultant.

Result. $(X Y+Y Z+Z X) \sqrt{ } 3+P(X+Y+Z)=0$; where $X, Y, Z$ denote the forces along the edges, and $P$ the force along the diagonal.

6. If a triangle is suspended from a fixed point by strings attached to the angles, the tension of each string is proportional to its length.

7. A uniform heavy triangle is supported in a horizontal position by three parallel strings attached to the three sides respectively; shew that there is an infinite number of ways 
in which the strings may be relatively disposed so that their tensiuns may, be equal, but that the nituation of one being given, that of each of the other two in determisate.

8. A sphere of given weight reats on three planes whose equations are

$$
\begin{array}{r}
x \cos \alpha+y \cos \beta+z \cos \gamma=0, \\
x \cos \alpha_{1}+y \cos \beta_{1}+z \cos \gamma_{1}=0, \\
x \cos \alpha_{1}+y \cos \beta_{2}+8 \cos \gamma_{1}=0,
\end{array}
$$

the axis of $z$ being vertical; find the prossure on each plane.

9. A henvy triangle $A B C$ is suspended from a point by three strings, mutually at right angles, attached to the angular points of the triangle; if $\theta$ be the inclination of the triang he to the horizon in its position of equilibrium, then

$$
\cos \theta=\frac{3}{\sqrt{(1+\sec A \sec B \sec C)}} \cdot
$$

10. An equilateml triangle without weight has three unequal particles placed at its angular points; the eystem is suspended from a fixed point by three equal atrings at rifht angles to each other fastemed to the corners of the triangle; find the inclination of the plane of the triangle to the harizan.

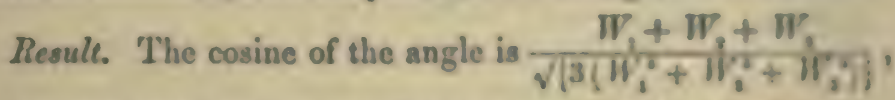
where $W_{3}, W_{2}, W_{2}$ represent the weights of the particless

11. Four amooth equal splieres are placed in a hemispherieal bowl. The centres of three of them are in the same herizontal plane, and that of the other is atove is. If the radius of each sphere be one-thiril that of the bowl, slicew that the mutual pressures of the spheres are all eqnal; and find the pressure of each of the lower gijeres on the bowl.

Risults. I.et $W$ be the weight of each of the spheres; then each of the mutual pressures between the spitaces is $\frac{W}{\sqrt{6}}$; and $\frac{4 W}{\sqrt{6}}$ is the pressure of each of the lower ophlieres ea the bowl. 
12. Three equal spheres hang in contact from a fixed point by three equal strings; find the heaviest sphere of given radius that may be placed upon them without causing them to separate.

Result. Let $W$ be the weight of each of the equal spheres, $\theta$ the angle which each string makes with the vertical, $\phi$ the angle which the line joining the centre of one of the three equal spheres with the centre of the upper sphere makes with the vertical; then the weight of the upper sphere must not $\operatorname{exceed} \frac{3 W \tan \theta}{\tan \phi-\tan \theta}$.

13. $A B C D$ is a tetrahedron in which the edges $A B, A C$, $A D$ are at right angles to each other; forces are represented in magnitude and direction by $A B, A C, A D, B C, C D, D B$ : determine their resultant.

14. Three equal hollow spheres rest symmetrically inside a smooth paraboloid of revolution, whose axis is vertical ; a solid sphere of equal radius is placed upon them: shew that the equilibrium will be destroyed if the radius of the spheres is less than $\frac{l}{2 \sqrt{6}}$, where $l$ is the latus rectum; the weight of the hollow spheres being neglected in comparison with that of the solid one. 


\section{(83)}

\section{CHA PTER VI.}

\section{retumanus of a cosstraised moter.}

86. To find the conditions of equilitirium of furces activg on a rigid body uncen one poine is fireal.

Let the fixed point be taken an the origin of co-prdinates. The action of the forces on the body will produce a pressary on the fixed point; let $\boldsymbol{X}, \boldsymbol{Y}, Z^{\prime}$ be the resolved jarts of this pressure parallel to the axes. Then the fixed point will exert forces $-X^{\prime},-Y^{\prime \prime},-Z^{\prime}$, againat the boly; and if we take theae forces in comnexion with the giren forees, we may stuppose the body to be free, and the equations of ergulibrina are

$$
\begin{aligned}
\mathbb{X}-X^{\prime} & =0, & \mathbb{Y} Y-Y^{\prime} & =0, & \mathbb{Z}-Z^{\prime} & =0, \\
L & =0, & M & =0, & N & =0 .
\end{aligned}
$$

The first three equations give the resolved parts of the pressure on the fixed point; and the last three are the cuils conditions to be antisfied by the given forces. Thus the foroes will be in equilitrium if the sums of the momenes of the forres weith respect te three straight lines at right angles to edoch other. and prassing thronght the fixed poinh, wanish.

Conversely, if the forces are in equilibrium the sum of the moments of the forces with respect to any straight line through the fixed point will vanish.

From the equations $X^{\prime \prime}=\mathbf{\Sigma} X, Y^{\prime \prime}=\mathbb{\Sigma} Y_{,}, Z^{\prime}=\mathbb{\Sigma} Z$, it follows that the pressure on the fixed point is equal to the msaltant of all the giren forces of the system moved parallel to themselves up to the fixed point.

If all the forces are parallel, we may take the axis of : passing through the fixed point parallet to the forces. Thiea all the forces included in $\Sigma X$ vanish, and so do all the forces included in $\Sigma Y$; thus $N$ vanishes, $M$ reduces to $-\Sigma Y_{a}$, and $L$ reduces to $\mathbf{X} z y$. Hence $\boldsymbol{X}^{\prime}$ and $Y^{\prime}$ vanish and the ejustions of equilibrium reduce to

$$
\mathbf{I} Z-Z^{\prime}=0, \quad \mathbf{\Sigma} Z y=0, \quad \mathbf{\Sigma} Z \boldsymbol{Z}=0 ;
$$


the first determines the pressure on the fixed point, and the other two are conditions which must be satisfied by the given forces.

If all the forces act in one plane passing through the fixed point, and we take this plane for that of $(x, y)$, all the forces included in $\mathbf{Z} Z$ vanish; also the ordinate parallel to the axis of $z$ of the point of application of each force is zero. Thus $L$ and $M$ vanish; also $Z$ vanishes, and the equations of equilibrium reduce to

$$
\mathbf{\Sigma} X-X^{\prime}=0, \quad \mathbf{\Sigma} Y-Y^{\prime}=0, \quad \mathbf{\Sigma}(Y x-X y)=0 ;
$$

the first two determine the pressure on the fixed point, and the third is the only condition which the forces must satisfy. Thus the forces will be in equilibrium if the sum of the moments of the forces with respect to the straight line perpendicular to their plane, and passing through the fixed point vanishes; and conversely, if the forces are in equilibrium the sum of the moments of the forces with respect to this straight line will vanish.

87. To find the condition of equilibrium of a body which has two points in it fixed.

Let the axis of $z$ pass through the two fixed points; and let the distances of the points from the origin be $z^{\prime}$ and $z^{\prime \prime}$. Also let $X^{\prime}, Y^{\prime}, Z^{\prime}$ be the resolved parts of the pressures on one point, and $X^{\prime \prime}, Y^{\prime \prime}, Z^{\prime \prime}$ those on the other point.

Then, as in Art. 86, the equations of equilibrium will be

$$
\begin{gathered}
\Sigma X-X^{\prime}-X^{\prime \prime}=0, \quad \Sigma Y-Y^{\prime}-Y^{\prime \prime}=0, \quad \Sigma Z-Z^{\prime}-Z^{\prime \prime}=0, \\
L+Y^{\prime} z^{\prime}+Y^{\prime \prime} z^{\prime \prime}=0, \quad M-X^{\prime} z^{\prime}-X^{\prime \prime} z^{\prime \prime}=0, \quad N=0 .
\end{gathered}
$$

The first, second, fourth, and fifth of these equations will determine $X^{\prime}, X^{\prime \prime}, Y^{\prime}, Y^{\prime \prime}$; the third equation gives $Z^{\prime}+Z^{\prime \prime}$, shewing that the pressures on the fixed points in the direction of the line joining them are indeterminate, being connected by one equation only. The last is the only condition of equilibrium, namely $N=0$. Thus the forces will be in equilibrium if the sum of the moments of the furces with respect to the straight line passing through the fixed points vanishes; and conversely, if the forces are in equilibrium the sum of the moments of the forces with respect to this straight line will vanish. 
89. The indeterminateness which oecura on to the values of $Z^{\prime}$ and $Z^{\prime \prime}$ might have been expected; for if twe forces, $-Z$ and $-Z^{\prime}$, act on a rigid body in the mame struight line, their effect will be the same at whatever point in their line of action we suppose them applied, and eonsepuently they may be supposed both to act at the same point, or one of them to be increased provided the other be equally diminished. If it be objected that in myy experimental case there really would be some definite preasure at each fixed point, we must reply, that no body on which we can experiment fulfils the condition of perfect rigility, on which our conclusions depend. See Ibineon, Art. 270 ; and Rinsot, Arta. 128-132.

The case which we have been considering is that of a body which is capable of turning round a fixed axis; for an axis will be fixed if two of its points are fixed.

89. If the holy, instend of having two fixed points, can turn round an axis and also slude aleng is, then in addition to the condition $N=0$, we mast have $\Sigma Z=0$, supposing the axis of a directed along the straight line on which the bods can turn and slinte. For the axis will not be able, an in the last case, to furnish any forces $-Z$ and $-Z^{\prime \prime}$ to counteract $\Sigma Z$, and therefore $\mathbf{\Sigma} Z$ must $=0$.

90. To find the conditions of equilibrium of a rigial butly resting on a smouth plane.

Iet this plane be the plane of $(x, y)$; and let $x^{\prime}, y^{\prime}$ bo the co-ordinates of one of the points of contact, $\mathbb{K}^{\prime}$ the prosaturs which the body exerts against the plane at that point. Then the force $-R^{\prime}$, and similar forces for the other points of contact, taken in connexion with the giren forees, ought to satisfy the equations of equilibrium; hence

$$
\Sigma X=0, \Sigma Y=0, \Sigma Z-R^{0}-R^{\prime \prime}-\ldots=0,
$$

$$
L-R^{\prime}-K^{\prime} y^{\prime \prime}-\ldots=0, \quad M+R x^{\prime}+R^{\prime \prime} x^{\circ}+\ldots=0, \quad N=0 \text {. }
$$

If only one point be in contact with the plane, then the third oquation gives the pressure, and we have five equations of condition,

$$
\mathbf{S}=0, \quad \mathbf{Y}=0, \quad L-y^{\prime} \mathbf{Z} Z=0, \quad M+x^{\prime} \mathbb{S} Z=0, \quad N=0 .
$$


If two points be in contact, then the equations

$$
\begin{array}{ll} 
& R^{\prime} y^{\prime}+R^{\prime \prime} y^{\prime \prime}=L, \quad R^{\prime} x^{\prime}+R^{\prime \prime} x^{\prime \prime}=-M, \\
\text { give } & R^{\prime}=\frac{L x^{\prime \prime}+M y^{\prime \prime}}{y^{\prime} x^{\prime \prime}-x^{\prime} y^{\prime \prime}}, \quad R^{\prime \prime}=-\frac{L x^{\prime}+M y^{\prime}}{y^{\prime} x^{\prime \prime}-x^{\prime \prime} y^{\prime \prime}} ;
\end{array}
$$

and the equations of condition are

$$
\Sigma X=0, \Sigma Y=0, \Sigma Z-\frac{L\left(x^{\prime \prime}-x^{\prime}\right)+M\left(y^{\prime \prime}-y^{\prime}\right)}{y^{\prime} x^{\prime \prime}-x^{\prime} y^{\prime \prime}}=0 \text {, and } N=0 \text {. }
$$

If three points are in contact, then the pressures are determined from the equations

$$
\begin{gathered}
R^{\prime}+R^{\prime \prime}+R^{\prime \prime \prime}=\Sigma Z, \\
R^{\prime} y^{\prime}+R^{\prime \prime} y^{\prime \prime}+R^{\prime \prime \prime} y^{\prime \prime \prime}=L, \\
R^{\prime} x^{\prime}+R^{\prime \prime} x^{\prime \prime}+R^{\prime \prime \prime} x^{\prime \prime \prime}=-M,
\end{gathered}
$$

and the conditions of equilibrium are

$$
\Sigma X=0, \quad \Sigma Y=0, \quad N=0 .
$$

If more than three points are in contact, then the pressures are indeterminate, since they are connected by only three equations; but the conditions of equilibrium are still

$$
\Sigma X=0, \quad \Sigma Y=0, \quad N=0 .
$$

91. The equations at the commencement of the preceding Article shew that if a body rests in equilibrium against a plane, the forces which press it against the plane must reduce to a single force acting in a direction perpendicular to the plane, for the condition

$$
L \Sigma X+M \Sigma Y+N \Sigma Z=0
$$

is satisfied, since $\Sigma X, \Sigma Y$, and $N$ vanish. Hence the forces reduce to a single force; and since $\Sigma X$ and $\Sigma Y$ vanish, this force must be perpendicular to the fixed plane.

Also, this single force must counterbalance the forces $-R^{\prime},-R^{\prime \prime} . .$, which are all parallel and all act in the same direction. Hence, from considering the construction given in Art. 66 for determining the centre of a system of parallel forces, it follows that the point where this resultant cuts the plane must be within a polygon, formed by so joining the points of contact as to include them all and to have no re-entering angle. 


\section{MISCRI.L.AEOUS F.X.MTLES.}

1. The lid ABCD of a cubieal lux, moveable aleus hinges at $A$ and $B$, is held at on given nngle to the herizin by a horizontal string connecting $C$ with a point vertically over A: find the pressure on ench linge.

2. Two equal forces act on a cube whose centre in fixed, along diagonals which do not meet of two adjacent faces: find the couple which will keep the cule at reat.

Resuls. Let $P$ denote each force, a the edge of the cubs; the mument of the required couple is either $\frac{\pi \sqrt{3}}{2}$ or $\frac{\pi}{2}$ ancurding to the directions of the two given forces.

3. Three equal heavy rods in the position of the throe elges of an inverted triangular pyramil are in equithbetum under the following circunstances: their upper extremitis are connected by strings of equal lengthe, and their lower extremities are attached to a hinge about which the gods may move freely in all directions. Find the tension of the strings.

4. A given number of uniform heary rods, all of the same weight, have their extremities jointed together at a common hinge, about which they cas turn froely; and being introduced through a circular hole in a horizontal plane with their hinge end downwards, are spread out symmetrically along the circumference of the hole like the rith of a conical basket. If a heavy sphere be now placed in the interior of the system of rods, so as to be supported by thesa, determine the position of rest.

5. A cylinder with its base resting againat a mischt vertical plane is held up by a string fantened to it at a foist of its curved surface whome distance from the vertieal folase is $h$. Shew that $h$ must be greater than $b-2 a \tan \theta$ and hes than $b$, where $2 b$ is the altitado of the cylindes, a the ralis of the base, and $\theta$ the angle which the string ruates wits ase vertical. 
6. A cylinder rests with its base on a smooth inclined plane; a string attached to its highest point, passing over a pully at the top of the inclined plane, hangs vertically and supports a weight; the portion of the string between the cylinder and the pully is horizontal. Determine the conditions of equilibrium.

Results. Let $W$ be the weight of the cylinder, $W^{\prime}$ the weight attached to the string, $\alpha$ the inclination of the plane to the horizon; then $W^{\prime}=W \tan \alpha$, and $\tan \alpha$ must not exceed the ratio of the diameter of the base of the cylinder to the height of the cylinder.

7. A cone of given weight $W$ is placed with its base on an inclined plane, and supported by a weight $W^{\prime \prime}$ which hangs by a string fastened to the vertex of the cone and passing over a pully in the inclined plane at the same height as the vertex. Determine the conditions of equilibrium.

Results. Let $\alpha$ be the inclination of the plane to the horizon, $\theta$ the semi-vertical angle of the cone; then

$$
W^{\prime}=W \tan \alpha \text {, and } \tan \theta \text { must not be less than } \frac{3}{8} \sin 2 \alpha \text {. }
$$

8. A smonth hemispherical shell whose base is closed includes two equal spheres whose radii are one third of that of the shell. The shell is fixed with its base vertical; find the mutual pressures at all the points of contact.

Results. Let $R_{2}$ be the pressure between the upper sphere and the shell, $R_{\mathrm{g}}$ that between the two spheres, $R_{\mathrm{s}}$ that between the lower sphere and the base of the shell, $R_{4}$ that between the lower sphere and the curved part of the shell; then

$$
R_{1}=\frac{W}{\sqrt{ } 3}, \quad R_{8}=\frac{2 W}{\sqrt{ } 3}, \quad R_{8}=\frac{3 W}{\sqrt{ } 3}, \quad R_{4}=\frac{4 W}{\sqrt{ } 3} .
$$

9. A rectangular table is supported in a horizontal position by four legs at its four angles: a given weight $W$ being placed upon a given point of it, shew that the pressure on each leg is indeterminate, and find the greatest and least value it can have for a given position of the weight. 


\section{(89)}

\section{CHAPTER VII.}

\section{GESERAL TIEOREMS ON A SYSTEAT OP PORERA.}

92. Is Art. 72 it is proved that the forces acting on a rigid body may be reduced to a force $I R$ and a couple $Z$, and that $G^{2}=L^{2}+M^{2}+N^{3}$, where $L_{2}, M, N$ are the moments of the forces round three rectangular axes arbitranily chisen. It is obrious that neither $L, M$, nor $\boldsymbol{N}$ can be grenter than $G$; lenoe, for a given origin, the resultant moment $G$ is greater than the moment of the furces about any other aris. Ias this reason $G$ is called the principal moment of the forces.

From the equations in Art. 72 , which determine the direetion of the axis of $G$, it follows that $G$ cos $\phi$ in the moment of the forces about an axis which pransen through the given origin, and makes an angle $\phi$ with the axis of principal moment.

93. The value of $R$ in Art. 72 is independent of the position of the origin of co-ordinates; $\mathbb{R}$ is in fact the tosultant of the given forces, supposing cach of them moved parallel to itself until they are all brought to act at the same point. The value of $G$, however, depends on the origin we assume. If we take a point whose co-ordinates are $x, y, z$, and denote by $L, M, N$, the moments of the fores nves straight lines through this point parallel to the co-ordisate axes, and by $G$ the principal moment of the forces with renject to this point, we have, by Art. 81 ,

$$
\begin{aligned}
& \boldsymbol{I}^{\prime}=L-\mathbf{y}^{\prime} \mathbf{Z} Z+\mathbf{z}^{\prime} \mathbf{Z} \mathbf{Y}, \\
& M=M-z^{\prime} \mathbf{I}+\boldsymbol{X}+\mathbb{Z}^{\prime}, \\
& \boldsymbol{N}^{\prime}=\boldsymbol{N}-\boldsymbol{x}^{\prime} \mathbf{\Sigma} \boldsymbol{Y}+\boldsymbol{y}^{\prime} \mathbf{\Sigma} \boldsymbol{X}, \\
& G^{n}=L^{m}+M^{n}+N^{n} \text {. }
\end{aligned}
$$


We proced to apply these equations to find the least value of $G^{\prime}$.

To find the locus of the origins which give the least principal moments, the magnitude of those moments, and the position of their axes.

Multiply the first of equations (1) by $\Sigma X$, the second by $\Sigma Y$, and the third by $\Sigma Z$, and add; thus

$$
L^{\prime} \Sigma X+M \Sigma Y+N^{\prime} \Sigma Z=L \Sigma X+M \Sigma Y+N \Sigma Z \ldots \text { (2). }
$$

Also

$$
\begin{aligned}
R^{2} G^{\prime 2} & =\left\{(\Sigma X)^{2}+(\Sigma Y)^{2}+(\Sigma Z)^{2}\right\}\left\{L^{\prime 3}+M^{2}+N^{\prime 2}\right\} \\
& =\left(N^{\prime} \Sigma Y-M^{\prime} \Sigma Z\right)^{2}+\left(L^{\prime} \Sigma Z-N^{\prime} \Sigma X\right)^{2} \\
& +\left(M^{\prime} \Sigma X-L^{\prime} \Sigma Y\right)^{2}+\left(L^{\prime} \Sigma X+M^{\prime} \Sigma Y+N^{\prime} \Sigma Z\right)^{2} \ldots \text { (3). }
\end{aligned}
$$

Of these four terms the last is constant for all values of $x^{\prime}, y^{\prime}, z^{\prime}$ by $(2)$; hence we obtain the least value of $G^{\prime}$ by making the three preceding terms vanish, which gives

that is,

$$
\frac{L^{\prime}}{\Sigma X}=\frac{M^{\prime}}{\Sigma Y}=\frac{N^{\prime}}{\Sigma Z} \ldots \ldots \ldots \ldots \ldots . . . . .(4) ;
$$

$$
\frac{L-y^{\prime} \Sigma Z+z^{\prime} \Sigma Y}{\Sigma X}=\frac{M-z^{\prime} \Sigma X+x^{\prime} \Sigma Z}{\Sigma Y}=\frac{N-x^{\prime} \Sigma Y+y^{\prime} \Sigma X}{\Sigma Z} \ldots(5) \text {. }
$$

Hence the required locus is a straight line.

From (4) it appears that $L^{\prime}, M^{\prime}, N^{\prime}$ are proportional to $\Sigma X, \Sigma Y, \Sigma Z$ respectively, which shews that the axis of the principal moment at any point on the straight line (5) is parallel to the direction of the resultant $R$. By (3) the value of the least principal moment is

$$
\frac{L \Sigma X+M \Sigma Y+N \Sigma Z}{\bar{R}} .
$$

Each of the fractions in (5) is, by a known theorem, equal to

that is, to

$$
\begin{aligned}
& \frac{L \Sigma X+M \Sigma Y+N \Sigma Z}{(\Sigma X)^{2}+(\Sigma Y)^{2}+(\Sigma Z)^{2}} \\
& \frac{L \Sigma X+M \Sigma Y+N \Sigma Z}{R^{2}}
\end{aligned}
$$


The equations (b) may by muitable transformations be reduced to the ordinary symmetrical equations to a straight line. We have

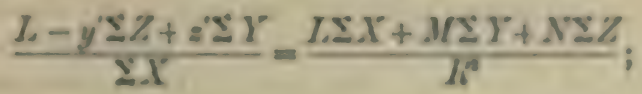

therefore

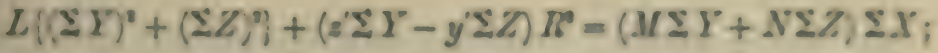

therefore

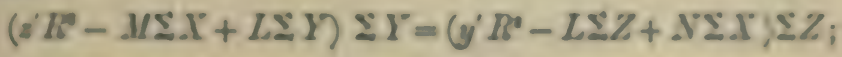

therefore

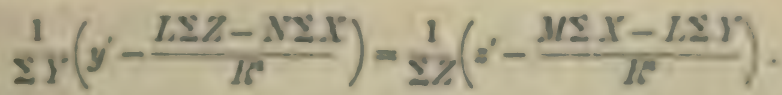

Hence we conclude that the equations (3) may be writte:

$$
\begin{aligned}
& \frac{1}{\Sigma X}\left(x^{\prime}-\frac{N \Sigma Y-M I Z}{10}\right)=\frac{1}{2 Y}\left(y^{\prime}-\frac{I \Sigma Z-N \mathbb{X} X}{16}\right) \\
& =\frac{1}{\Sigma Z}\left(z-\frac{M \mathbb{X} X-L \Sigma Y}{K}\right):
\end{aligned}
$$

from which we sce that the straight line determined by $(b)$ is parallel to the direction of $R$. Hence this straight line has the following properties: at every point of it the value of the principal moment is the same, and is less than it os fur any print not in the line; also for etery point in the line the pasitim of the axis of principal mement is the same, keing the lind itself. This line is called the central axis.

We have supposed in the investigntion that $\mathbb{R}$ is not zero. If $l l$ be zero we have for every origin

$$
\begin{gathered}
L^{\prime}=L, \quad M^{\prime}=M, \quad N^{\prime}=N, \\
G^{3}=L^{2}+M^{2}+N^{2} .
\end{gathered}
$$

94. The equation (2) of Art. 93 may be written

$$
L \frac{\Sigma X}{R}+M \frac{\Sigma Y}{16}+N \frac{\Sigma Z}{16}=L \frac{\Sigma X}{16}+M \frac{\Sigma Y}{16}+N \frac{\Sigma Z}{16} \text {. }
$$


This shews that if we resolve $L, M, N^{\prime}$ along a straight line parallel to the direction of $R$, and add the resolved parts, we obtain the same result whatever origin be chosen. Thus the resolved part of any principal moment in the direction of $R$ is constant. By the resolved part of the principal moment in the direction of $R$ we mean that part of the moment which has its axis in the direction of $R$.

95. From equations (1) of $\Lambda$ rt. 93 it appears that $L^{\prime}=L$, $M^{\prime}=M$, and $N^{\prime}=N$, provided

$$
\frac{x^{\prime}}{\Sigma X}=\frac{y^{\prime}}{\Sigma Y}=\frac{z^{\prime}}{\Sigma Z^{\prime}} ;
$$

that is, if the point $\left(x^{\prime}, y^{\prime}, z^{\prime}\right)$ be on a straight line through the origin parallel to the direction of $R$. Since the origin is arbitrary, we may therefore assert that the principal moment remains unchanged, when the point to which it relates moves along any straight line parallel to the direction of $R$.

96. The equation to the plane through the origin perpendicular to the direction of $R$ is

$$
x^{\prime} \Sigma X+y^{\prime} \Sigma Y+z^{\prime} \Sigma Z=0 \text {. }
$$

If we combine this equation with equations (5) of Art. 93, we obtain the co-ordinates of the point of intersection of this plane with the central axis.

We thus find for these co-ordinates

$$
\frac{N \Sigma Y-M \Sigma Z}{H^{n}}, \frac{L \Sigma Z-N \Sigma X}{K^{3}}, \frac{M \Sigma X-L \Sigma Y}{H^{x}},
$$

which we will denote by $h, k, l$ respectively.

$$
\text { If } x^{\prime}, y^{\prime}, z^{\prime} \text { satisfy (1), then } N^{\prime} \Sigma Y-M^{\prime} \Sigma Z
$$

$$
\begin{aligned}
\left(N-x^{\prime} \Sigma\right. & \left.Y+y^{\prime} \Sigma X\right) \Sigma Y-\left(M-z^{\prime} \Sigma X+x^{\prime} \Sigma Z\right) \Sigma Z \\
= & N^{\prime} \Sigma Y-M I Z-x^{\prime} R^{2}=R^{2}\left(h-x^{\prime}\right) . \\
& L^{\prime} \Sigma Z-N^{\prime} \Sigma X=R^{2}\left(k-y^{\prime}\right), \\
& M^{\prime} \Sigma X-L^{\prime} \Sigma Y=R^{2}\left(l-z^{\prime}\right) .
\end{aligned}
$$

Similarly 
Therefore from equation (3) of Art. 93

$$
\begin{aligned}
& G^{\prime 2}=M^{\prime}\left(\left(h-x^{2}\right)^{3}+\left(k-y^{\prime}\right)^{0}+\left(Z-z^{\prime}\right)\right\}
\end{aligned}
$$

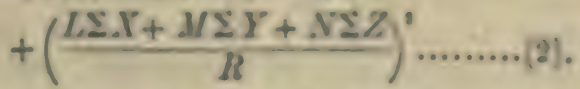

Hence $G^{\prime}$ remains constant for all points in the plane (1) for which $\left(h-x^{2}\right)^{3}+(k-y)^{3}+\left(l-z^{2}\right)^{3}$ is constant? that is, for all points in (1) which are at a constant diatance from the central axis. From this and Art. 93 it follows, that if a refhe cylinder be described round the central aris, the primeral momint hats the same value for any point on the surfuce of this cylinder.

97. Of the two expressions which compose $G^{\prime}$ in equation (2) of Art. 96, the latter, by Art. 94, is the reolved part of $G$ perallel to the direction of $R$; hence the former part is the resolved part of $G$ perpendicular to the direction of $R$. Call the former part $Q$, and $\phi$ the angle which the dirsction of the axis of $C^{\prime}$ makes with that of $R$; then sin $\phi=\frac{Q}{G^{\gamma}}$, and this is constant so Inng as $G^{\prime}$ is, that is, fer every point on the surfuce of the cyliniler in the preceding Article.

98. The propositions already given in this Chapeer admit of other modes of demonstration, which we prueced to indicate.

To shew that any system of forces can alioays be reilined to a force and a couple, the axis of the latter being parrallit to the direction of the former.

The firces can be always reduced to a force $R$ and a cuppla $G$, and the angle $\phi$ between the former and the axis of the latter is given by the equation

$$
\cos \phi=\frac{L \Sigma X+M \mathbb{I} Y+N \leq Z}{(i \cdot M} .
$$

Resolve the couple $G$ into two others; one hariag its asis parallel to the direction of $\mathbb{R}$ and its moment equal to $6 \mathrm{cos} \delta$. the other having its axis perpendicular to the dircetion of $f f$ and its moment equal to $G \sin \phi$. The fonors of the laters couple are therefore in a plane parallel to $\delta_{\text {; }}$ and ly pos- 
perly placing this couple in its own plane; and making each of its forces equal to $R$, one of its forces may be made to balance the force $R$. We shall then have remaining the couple $G \cos \phi$ and a force $R$, the direction of which is parallel to the axis of the couple, and which is moved to a distance $\frac{G \sin \phi}{l}$ from its original position. The system is thus reduced to a force $R$ and a couple $\frac{L \Sigma X+M \Sigma Y+N \Sigma Z}{R}$, the axis of the latter being parallel to $R$, and therefore its plane perpendicular to $R$.

Since the resultant couple must be independent of the direction of the axes of co-ordinates we conclude that

$$
\frac{L \Sigma X+M \Sigma Y+N \Sigma Z}{R}
$$

must be constant whatever be the direction of the axes; and as $R$ is constant it follows that $L \Sigma X+M \Sigma Y+N \Sigma Z$ must be constant whatever be the direction of the axes. The expression also remains the same whatever origin be chosen, as appears from equation (2) of Art. 93.

99. When a system of forces is reduced to a force and a couple in a plane perpendicular to the force, the position and magnitude of the force are always the same.

The magnitude of the force is always the same, for it is the resultant of the given forces supposing each of them moved parallel to itself until they are all brought to act at the same point. We shall now shew that there is a definite straight line along which the resultant force must act.

Let $x^{\prime}, y^{\prime}, z^{\prime}$ be the co-ordinates of an origin such that the axis of the resultant couple coincides with the direction of the resultant force. Then, with the notation of Art. 93, we have

$$
\frac{L^{\prime}}{\sum X}=\frac{M^{\prime}}{\sum Y}=\frac{N^{\prime}}{\Sigma Z^{\prime}},
$$

for the direction cosines of the axis of the couple are proportional to $L^{\prime}, M^{\prime}$, and $N^{\prime}$, and those of the direction of the 
force are propartional to $\mathbf{\Sigma} X, \mathbf{Z}, \mathbf{Z} Z$. Hence the lerus of the origins is the straight line determined by equations (6) of Art. 93.

100. It appears from the last Article that them is chaly one position of the resultant forat in which it is perpendienlar to the plane of the resultant couple. If we wish to transfer the resultant force to any other point, we can do it by introdncing two forces, $R$ and $-R$, at that point; the latter with the original force $R$ will form a couple; and if this couple be compounded with the original couple we have a new couple, the moment of which is $\sqrt{ }\left(K^{3}+\Gamma_{p}\right)$, where $K$ denotes the original moment and $p$ the distance to which $\boldsymbol{K}$ has been noved. This moment is greater than $K$; and hence the straight line in which $R$ nets when perpendicular to the plane of the resultant couple is the axio of least primcijal monent. It is therefore the central axis.

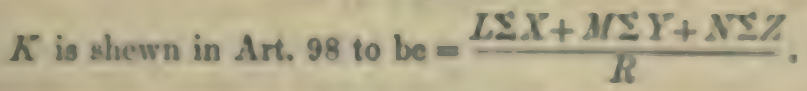

101. The principal moment will be the same for every point of the central axis, since when we have redued the furces to a single force and a couple in a plane perpendicular to the firce, the force may be supposed to act at any point in its line of application, and the plane of the couple may be moved parallel to itself into any new position. See also Ari. 95. Hence if we draw any plane perpendicular to the central axis. and describe a circle in the plane with radius $p$, and having its centre at the intersection of the central axis, then, by the Jast Article, the principal moment for any point in this circle will be $\sqrt{ }\left(K^{3}+K^{2} p^{2}\right)$, and the angle $\phi$ at which the direction of its axis is inclined to the direction of $\mathbb{R}$ is given by the equation $\tan \phi=\frac{R p}{K}$.

102. When a system of forces acting on a rigiel bos?y is reduced to tioo forces, and these gre represented by thoo struight lines achich do not meet and are not parallel, the nolume of the tetrahedron of which the tero straight lines are oppwaite aljes is constane. 
Let the straight lines $A B$ and $A^{\prime} B^{\prime}$ represent the two forces, $A A^{\prime}$ being a straight line at right angles to both. Suppose two parallel lines $A x, A^{\prime} x^{\prime}$ drawn, each at right angles to $A A^{\prime}$, and $A y, A^{\prime} y^{\prime}$, respectively at right angles to $A x$, $A^{\prime} x^{\prime}$, and also at right angles to $A A^{\prime}$. Let $B A x=\phi, B A^{\prime} x^{\prime}=\phi^{\prime}$, and let $T$ and $T^{n}$ denote the intensities of the forces in $A B$ and $A^{\prime} B^{\prime}$

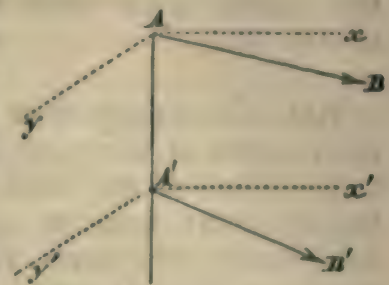
respectively. Then $T$ may be resolved into $T \cos \phi$ and $T^{\prime} \sin \phi$ acting at $A$ along $A x$ and $A y$ respectively, and $T^{\prime \prime}$ into $T^{\prime \prime} \cos \phi^{\prime}, T^{\prime \prime} \sin \phi^{\prime}$ acting at $A^{\prime}$ along $A^{\prime} x$ and $A^{\prime} y^{\prime}$ respectively. Let $\alpha$ be the inclination of $A B$ and $A^{\prime} B^{\prime}$, so that $\phi^{\prime}=\phi+\alpha$. Now determine $\phi$ by the equation

$$
T \cos \phi+T^{\prime \prime} \cos \phi^{\prime}=0 \text {. }
$$

that is $\quad T \cos \phi+T^{\prime \prime} \cos (\phi+\alpha)=0$.

Then by (1) the forces $T \cos \phi$ and 'T' $\cos \phi^{\prime}$ will form a couple in the plane $x A A^{\prime} x^{\prime}$; and $T \sin \phi$ and $T^{\prime \prime} \sin \phi^{\prime}$ will have a single resultant perpendicular to the plane of this couple, for they cannot form a couple since then the whole system of forces would reduce to a single couple which is contrary to supposition. Let $P$ denote the intensity of this single force so that

$$
P=T \sin \phi+T^{\prime} \sin \phi^{\prime} .
$$

The moment of the couple is $A A^{\prime} \times T \cos \phi$. Hence, by the latter part of Art. 98, $A A^{\prime} \times P \times T \cos \phi$ is constant whatever be the position and magnitude of the forces $T$ and $T^{\prime \prime}$, so long as they are equivalent to a given system of forces.

Now the volume of the tetrahedron of which $A B$ and $A^{\prime} B^{\prime}$ are opposite edges is $\frac{1}{6} A B \cdot A^{\prime} B^{\prime} \cdot A A^{\prime} \sin \alpha$. For the base may be considered to be the triangle $A A^{\prime} B^{\prime}$, the area of which is $\frac{1}{2} A A^{\prime} \cdot A^{\prime} B^{\prime}$; and the height will then be $A B \sin \alpha$.

But from (1) and (2) we have $T^{\prime \prime} \sin \alpha=P \cos \phi$. Hence the volume of the tetrahedron becomes $\frac{1}{d} A A^{\prime} . T \cdot P \cos \phi$, which we have just seen to be constant.

This result is due to Chasles; see Möbius, Lehrbuch der Statik, I. 122. 
103. When a system of parallel forees acting on a ripid body has a single resnltant, that resuleant always passes throngh a fixed point in the body whatever may be the position of the body. When any system of furces acts on a rigid boly we might investigate the conserquences of turning the body from one position into noocher while the forces retain their original directions, or of turning the forcen in ouch a manner as to leave their relative directions unchanged while the body remains fixed. We shall here give some examples of the general theorems that have been demonstrated on this subject. The forces are supposed to act at fixed points in the body.

104. Let $P A$ and $Q A$ be the directions of two forees lying in one plane, acting at the points $P$ and $Q$ respectively; $T A$ the direction of their resultant. Suppose the forces in $P A, Q A$ to be turned round the points $P$ and $Q$ respectively through the same angle $\alpha$ towards the same direction; since $P A$ and $Q .4$ will include the same angle as before, their point of interscction will move on a circle passing through $P$ and $Q$. And

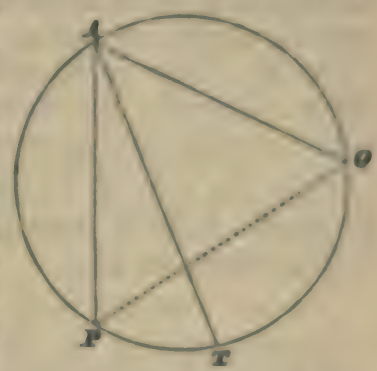
is the magnitudes of the forces are supposed unchanged, the magnitude of the resultant and the angles which it makes with the components remain unchanged. Hence if $T$ be the intersection of the resultant and the circle originally, it will always be so, since the arcs $P T$ and $Q T$ are proportional to the angles $P A T$ and $Q A T$; the resultant will therefore have turned through the angle a round the point $T$.

The same conclusion holds if instead of supposing the body in be fixed and the forces to revolve, we suppose ench force to remain parallel to itself and the body to be curned through any angle round a perpendicular to the plane of the forces.

The point $T$ through which the resultant always pases may be called the centre of the forces which act at $P$ and $Q$. It is evident, in like manner, that if a third foree posa through a fixed point $S$ and meet the straight line $T A$, we 
may find the centre of the forces at $T$ and $S$, that is, the centre of the forces at $P, Q$, and $S$; and generally we may infer that every system of forces in one plane which is reducille to a single resultant has a centre; or, in other words, if there be a system of forces acting in a plane and having a single resultant, and we know the magnitude of each force, the angles the directions of the forces make with each other, and one point in the direction of each, then we can determine the magnitude of the resultant, the angle its direction makes with those of the component forces, and one point in its direction.

105. If a system of forces maintain a body in equilibrium, and equilibrium also subsist after the body has been turned through any given angle which is not a multiple of two right angles, about any axis, then equilibrium will still subsist when the body is turned about the same axis through any angle whatever, the forces being supposed to act with the same intensity and in parallel directions throughout.

Take the axis of $z$ to coincide with the straight line about which the body is turned. Since there is equilibrium in its first position, we have

$$
\begin{array}{r}
\mathbf{\Sigma} X=0, \quad \mathbf{\Sigma} Y=0, \quad \mathbf{\Sigma} Z=0 \ldots \ldots \ldots \ldots \ldots(1), \\
\mathbf{\Sigma}\left(Z y-Y_{z}\right)=0, \mathbf{\Sigma}\left(X_{z}-Z x\right)=0, \mathbf{\Sigma}\left(Y_{x}-X_{y}\right)=0 \ldots(2) .
\end{array}
$$

If equilibrium subsist when the body is turned through an angle $\theta$, the equations (1) and (2) must hold when we put $r \cos \theta-y \sin \theta$ for $x$, and $x \sin \theta+y \cos \theta$ for $y$. Hence (2) become

$$
\begin{aligned}
\sin \theta \Sigma(Z x)+\cos \theta \Sigma(Z y)-\Sigma(Y z) & =0 \ldots \ldots(3), \\
\Sigma(X z)-\cos \theta \Sigma(Z x)+\sin \theta \Sigma(Z y) & =0 \ldots \ldots(4), \\
\cos \theta \Sigma(I x-X y)-\sin \theta \Sigma(X x+Y y) & =0 \ldots \ldots(5) .
\end{aligned}
$$

By means of (2), equations (3) and (4) become

$$
\begin{aligned}
& \sin \theta \mathbf{\Sigma}\left(X_{z}\right)-(1-\cos \theta) \Sigma\left(Y_{z}\right)=0, \\
& (1-\cos \theta) \Sigma\left(I_{z}\right)+\sin \theta \mathbf{\Sigma}\left(I_{z}\right)=0 .
\end{aligned}
$$

As these equations hold for some value of $\sin \theta$ different from zero we must have

$$
\Sigma\left(X_{z}\right)=0 \text {, and } \Sigma\left(Y_{z}\right)=0 \text {. }
$$




\section{A sหstis or roteces}

Then, by (2), we infer

$$
\Sigma(Z x)=0, \text { and } \mathbf{\Sigma}\left(Z_{y} y\right)=0
$$

And from (2) and (5),

$$
\mathbf{I}\left(J_{x}-X_{y}\right)=0 \text {, and } \mathbf{I}\left(X x+J_{y}\right)=0 \ldots \ldots \text { (s). }
$$

And when $(6)$, (7), and $(8)$ are true, $(3),(1)$, and $(9)$ are true fing ull values of $\theta$.

It nppears from the preceling insestigation that when forres act in one pleme on a rigid body and maintin equihisim, the necseary and sufficient alditional condition in orler that egnilibrimm may subsist after the hoty las been curned rotand an axis perpendicular to the plane while the forces remain parallel to their original directons, is

$$
\Sigma(X x+Y y)=0 \text {. }
$$

106. A system of furces aets on a ricid louly: determine the cotdituons which must hoid in erder that when the svat-m is rosolsed paralle to any straight line these resolved parts many bo in equilibrium.

Take a straight line whose direction cosines are $I, m, n$. In eriler that the resolved parts of the foross parallel to fis straight line may be in equilibrium we must have, by Art. 78 ,

$$
\begin{aligned}
& \Sigma(l X+m Y+n Z)=0, \\
& \frac{\Sigma(L X+m Y+n Z) v}{l}=\frac{\mathbb{S}(l X+m Y+n Z) y}{m} \\
& =\frac{\mathbb{S}\left(L X+m \Gamma^{2}+n X\right):}{n} .
\end{aligned}
$$

And ns these are to be true fur all ratios of $l, m, n$ we must have

$$
\mathbf{I} \boldsymbol{X}=0, \quad \mathbf{Y}=0, \quad \mathbf{\Sigma} Z=0,
$$

$\Sigma Y_{y}=0, \Sigma X_{z}=0, \Sigma I_{x}=0, \Sigma I_{z}=0, \Sigma Z_{x}=0, \Sigma Z_{y}=0$,

$$
\Sigma X x=\Sigma Y y=\Sigma Z \text {. }
$$

These are the necessury and sufficient conditions. 
107. We have remarked in Art. 9 that the property of the divisibility of matter leads us to the supposition that every body consists of an assemblage of material particles or molecules which are held together by their mutual attraction. Now we are totally unacquainted with the nature of these molecular forces; if, however, we assume the two hypotheses that the action of any two molecules on each other is the same, and also that its direction is the straight line joining them, then we shall be able to deduce the conditions of equilibrium of a rigid body from those of a single particle.

To deduce the conditions of equilibrium of a rigid body from those of a single particle.

Let the body be referred to three rectangular axes; and let $x_{1}, y_{1}, z_{1}$ be the co-ordinates of one of its constituent particles; $X_{1}, Y_{1}, Z_{1}$ the resolved parts, parallel to the axes, of the forces which act on this particle exclusive of the molecular forces; $P_{1}, P_{s}, P_{3}, \ldots$. the molecular forces acting on this particle; $\alpha_{1}, \beta_{1}, \gamma_{1} ; \alpha_{y}, \beta_{2}, \gamma_{s} ; \ldots .$. the angles their respective directions make with the three axes of co-ordinates. Then, since this particle is held in equilibrium by the above forces, we have, by Art. 27,

$$
\begin{aligned}
& X_{1}+P_{2} \cos \alpha_{1}+P_{2} \cos \alpha_{2}+\ldots \ldots=0 \ldots \ldots(1), \\
& Y_{2}+P_{1} \cos \beta_{1}+P_{2} \cos \beta_{2}+\ldots \ldots=0 \ldots \ldots \text { (2), } \\
& Z_{1}+P_{1} \cos \gamma_{1}+P_{2} \cos \gamma_{2}+\ldots \ldots=0 \ldots \ldots \text { (3). }
\end{aligned}
$$

We shall have a similar system of equations for each particle in the body; if there be $n$ particles there will be $3 n$ equations. These $3 n$ equations will be connected one with another, since any molecular force which enters into one system of equations must enter into a second system; this is in consequence of the mutual action of the particles.

There are two conditions which will enable us to deduce from these $3 n$ equations six equations of condition, independent of the molecular forces. These will be the equations which the other forces must satisfy, in order that equilibrium may be maintained. 
The first endition is this, that the molecular actians are mutual; and that, conserquently, if $P_{3} \cos a_{1}$ represent the resolved part parallel to the axis of $x$ of way one of tive molecular forees involved in the $3 n$ equations, we aliall likewise meet with the term $-P_{1} \cos a_{3}$ in another of those equas. tions which have reference to the axis of $x$. Consegnently, if we aild all those equations together which have reference to the game axis, we have the three following equations of condition independent of the molecular furces,

$$
\Sigma X=0, \quad \Sigma Y=0, \quad \Sigma Z=0 .
$$

The socond consideration is this: that the stmight lines joining the diffirent particles are the directions in which the molecular forees act.

Thus, let $P_{1}$ be the molecular action between the particles whuse co-ordinates are $\left(x_{1}, y_{1}, s_{2}\right)$ and $\left(x_{2}, y_{2}, z_{2}\right)$,

$$
\begin{array}{rrr}
P_{1} \cos \alpha_{1}, & P_{1} \cos \beta_{1}, & P_{1} \cos \gamma_{1}, \\
-P_{1} \cos \alpha_{1}, & -P_{1} \cos \beta_{1}, & -P_{1} \cos \gamma_{1},
\end{array}
$$

the corresponding resolved parts of $P_{1}$ for the two particless. Then

$$
\cos \alpha_{1}=\frac{x_{2}-x_{3}}{r}, \cos \beta_{1}=\frac{y_{8}-y_{3}}{r}, \cos \gamma_{1}=\frac{z_{2}-\varepsilon_{3}}{r},
$$

where

$$
r=\sqrt{ }\left(\left(r_{2}-x_{2}\right)^{2}+\left(y_{2}-y_{2}\right)^{0}+\left(z_{3}-z_{2}\right){ }^{\prime}\right\}
$$

These enable us to obtain three more equations free from molecular forces: for if we multiply (1) and (2) by $y_{1}$ and $x_{1}$ tespectively, and then subtract, we have

$$
Y_{1} x_{3}-X_{2} y_{1}+\ldots+P_{1}\left[x_{1} \cos \beta_{1}-y_{1} \cos a_{2}\right)+\ldots=0 \ldots(t) \text {. }
$$

By the anme process we obtain from the system of eqguations which refer to the particle $\left(x_{0}, y_{2}, z_{3}\right)$,

$$
Y_{2} x_{2}-X_{2} y_{2}+\ldots-P_{1}\left|x_{2} \cos \beta_{1}-y_{2} \cos \alpha_{1}\right|+\ldots=0 \ldots \text { (3). }
$$

But the values of $\cos \alpha_{1}$ and $\cos \beta_{1}$ given above lead to the condition

$$
\left(x_{2}-x_{2}\right) \cos \beta_{1}-\left(y_{2}-y_{1}\right) \cos z_{1}=0 \text {. }
$$


Wherefore the equation

$$
Y_{1} x_{8}-X_{1} y_{1}+\ldots \ldots+Y_{8} x_{2}-X_{2} y_{8}+\ldots \ldots=0
$$

will not involve $P_{1}$, the molecular action between the particles whose co-ordinates are $x_{1}, y_{1}, z_{1}$ and $x_{8}, y_{s}, z_{s}$ respectively.

It follows readily from what we have shewn, that if we form all the equations similar to (4) and (5), and add them together, we shall have a final equation

$$
\Sigma(Y x-X y)=0
$$

independent of the molecular forces.

In like manner we should obtain

$$
\Sigma(Z y-Y z)=0, \quad \Sigma(X z-Z x)=0 .
$$

Moreover we can shew that these six equations are the only equations free from the molecular forces, supposing the body to be rigid, and consequently the molecules to retain their mutual distances invariable. For if a body consist of three molecules, there must evidently be three independent molecular forces to keep them invariable; if to these three molecules a fourth be added, we must introduce three new forees to hold it to the others; if we add a fifth molecule we must introduce three forces to hold this invariably to any three of those which are already rigidly connected; and so on; from which we sec that there must be at least $3+3(n-3)$ or $3 n-6$ forces. Hence the $3 n$ equations resembling (1), (2), and (3) contain at least $3 n-6$ independent quantities to be eliminated; and therefore there cannot be more than six equations of condition connecting the external forces and the co-ordinates of their points of application.

\section{MISCELLANEOUS EXAMPLES.}

1. Determine the central axis when there are two forces $P$ and $Q$ whose lines of action are defined by $z=c, y=x \tan \alpha$, and $z=-c, y=-x \tan \alpha$ respectively. 
2. If $P$ and $Q$ are two forees whose directions are at riplt migles, shew that the dietaneres of the central axis from thes lines of action are as $I^{n}$ to $C$.

3. Parallel foroes act on a rigid bonly and maintain it in equilibrium, the prints of applieation being all in one plane: shew that the forees will maintain the body in equihitrium however they may be turned about their points of application.

4. A system of forers acting on a rigid body is equiralent to a single fore : shew that it will also be equivalent to a singla fine aftar the boly has becn tarnal turumgh any arglo ahiut the axis of $\mathrm{s}$, the directions of the furces remaining the same, if

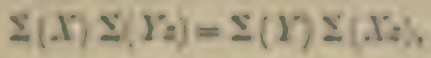

and $\mathbf{\Sigma}\left(X_{7} \mathbf{\Sigma}\left(Z_{x}\right)+\mathbf{\Sigma}(Y) \mathbf{\Sigma}\left(Z_{y}\right)=\mathbf{\Sigma}(a) \mathbf{\Sigma}\left(X_{x}+I_{y}\right)\right.$.

5. Forees act at the ancular points of a tetraliedron in directiven respectively perpendicular to the omposite faces, and proportional to the areas of the faces in magritule: shew that the forces have the property considered in $\mathrm{Art}$. $10 \mathrm{c}$

6. Shew that within a qualrilateral, no two siles of which are parallel, there is but one point, at which forces acting towards the corners and proportional to the distances of the point from them, can be in equililurium.

7. Two forces acting at a point are represented in magnitude and direction by straight lines drawn from that point their sum is constant and their resultant is enstant both is magnitude and direction. Find the lwous of the extremities of the straight lines which represent the furces.

8. If forces $P, Q, R$ acting at the centre $O$ of a circular lamina along the radii $O A, O B, O C$ be equivalent to foroes $I^{\prime}, Q, R$ acting along the sides $B \bar{C}, C . A, A B$ of the inseribed triangle, ahew that

$$
\frac{P \cdot P}{B U}+\frac{Q \cdot Q}{U A}+\frac{R \cdot \pi}{A B}=0 .
$$


9. A uniform rigid rod, of length $2 a$, can turn in a horizontal plane about its middle point. At one end a string is tied which passes over a fixed pully, vertically above that end, and at a distance $b$ from it, and is then fastened to a given weight. The rod is then turned through an angle $\theta$, and kept at rest in that position by a horizontal force $P$ perpendicular to the rod through its other end. Prove that $P$ will be a maximum if

$$
\tan ^{4} \frac{\theta}{2}=\frac{b^{2}}{b^{4}+4 a^{3}} .
$$

10. Prove that a system of forces can be reduced in an infinite number of ways to a pair of equal forces, whose directions make any assigned angle with one another; and find the distance between these forces when the angle is given. 


\section{CIATTER VIII.}

\section{CENTRE OF ORAVITX.}

105. Wercint is mensured like other quantities by means of an arbitrary unit. If a certain upward force be necesary to prevent a body from falling, then another body which ropuires an equal force to sustain it is said to have a weight cyual to that of the first. When two weights have been recognised to be equal, a body which requires to sustain it a force equal to the sum of the two equal forces which would sustain the two eyual weights, is said to have a weight double that of either of the two equal weights; and so on.

It appears from experiment that the weight of a given body is imvariatle so long as the body remains at the same place on the earth's surface, but changes when the boly is taken to a difterent place. Wo shall suppose therefore when we speak of the weight of a body that the body remains at one place.

When a body is such that the weight of any portion of it is proportional to the volume of that portion it is said to be of uniform density; the density of such a body is mesarured by the ratio which the weight of any volume of it beass to the weight of an equal volume of some arbitrarily chosen body of uniform density.

The product of the density of a body into its volume is called its mass.

When a body is not of uniform density its densify at any point is measured thus: find the ratio of the weight of a volume of the body taken so as to include that point to the weight of an equal volume of the standard bodyz the limit of this ratio, when the volume is inclefinitely diminished, is the density of the body at the assumed point. 
109. It was shewn in Art. 66 that there is a point in every body such that, if the particles of the body be acted on by parallel forees and this point be fixed, the body will rest in whatever position it be placed.

Now the weight of a body may be considered as the resultant of the weights of the different elementary portions of the body, acting in parallel and vertical lines. In this case the point above described as the centre of parallel forces is called the centre of gravity of the body. We may define the centre of gravity of any system of heavy particles as a point such that if it be supported and the particles rigidly connected with it, the system will rest in any position.

In the present Chapter we shall determine the position of the centre of gravity in bodies of various forms. We shall first give a few elementary examples.

(1) Given the centres of gravity of treo parts which compose a body, to find the centre of gravity of the whole body.

Let $G_{1}$ denote the centre of gravity of one part, and $G_{2}$ the centre of gravity of the other part; let $m_{3}$ denote the mass of the first part and $m_{2}$ the mass of the second part. Join $G_{1} G_{2}$ and divide it in $G$ so that $\frac{G G_{3}}{G G_{3}}=\frac{m_{9}}{m_{1}}$, then $G$ is the centre of gravity of the whole body (Art. 37).

(2) Given the centre of gravity of a body and also the centre of gravity of a part of the body, to find the centre of gravity of the remainder.

Let $G$ denote the centre of gravity of the body, and $G_{1}$ the centre of gravity of a part of the body; let $m$ denote the mass of the body, and $m_{1}$ the mass of the part. Join $G_{1} G$ and produce it through $G$ to $G_{n}$, so that $\frac{G G_{2}}{G G_{1}}=\frac{m_{1}}{m-m_{1}}$, then $G_{s}$ is the centre of gravity of the remainder.

(3) To find the centre of gravity of a triangular figure of uniform thickness and density. 
Let $A L C$ be one surface of the triangalar figure; Lisect EI? at $E$; join $A E$; draw cib paraliel to CEl eutsing $A f$ at $e$. Then, by similar triangles,

ce $C E$ : $A C: A E^{\circ}$

and bo: $B E$ : $A 0: A E$,

therefore ce : $C E$ :: le : $B E$;

but $C E=I B E$, therefore ce $=$ in.

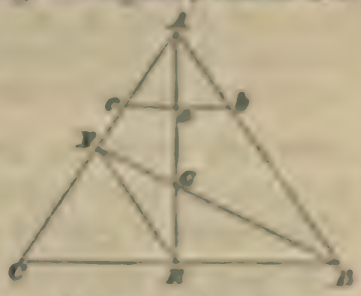

Henee $A B$ lieects every straight line parallel to $B C$. Ther.fure each of the strips similar to cob, into which wo may sumpose the triangle to be divided, will balance on $A E$, anit thenefure the centre of gravity must be in the straight line $A E$.

Bisect $A C$ at $F$ and join $B F$; let this cut $A F$ at $G$. Then, as before, the centre of grarity must be in $B F$; but it mut tee in $A \mathrm{k}$; and therefore $G$ is the centre of gravity.

Join $E F$. Then, because $C E=I B F$ and $C F=A F$, therefore $E F$ is parallel to $A B$ and $A B=2 F E$; and by similar triangles,

$$
E G: E F:: A G: A B \text {, therefore } E G=\frac{1}{d} A G \text {. }
$$

Hence to find the centre of gravity of a triangle, bisect any side, join the point of bistction with the opposite angles and the centre of gravity lies a third of the way up this straight line.

The centre of gravity of any plane polygon may be found by dividing it into triangles, determining the centre of gravicy. of each triangle, and then by Art. 66 deducing the eentre of gravity of the whole figure.

We may observe that the centre of gravity of a triangle coinciles with the centre of gravity of three equal particles placed at the angular points of the iriangle. For to find the centre of gravity of three equal particles placed at $A, B, O$ respretively, we join $C B$ and biseet it at $E$; then $E$ is the centre of gravity of the particles at $C$ and $B$ : suppose these particles oullected at $E$; then join $A E$ and divile $A E$ at $G$ so that $E G$ may be to $A G$ as the mass of the one particle at $A$ is to that of the two at $E$, that is, as 1 is to 2 ; then $G$ is the centre of gravity of the three equal particles. From the construction $F$ is obviously also the centre of gravity of the triangle $\triangle B C$. 
Let the co-ordinates of $A$ referred to any axes be $x_{1}, y_{1}, z_{1}$; those of $B, x_{2}, y_{2}, z_{2}$; and those of $C, x_{2}, y_{3}, z_{3}$; then, by Art. 66, the co-ordinates $\bar{x}, \bar{y}, \bar{z}$ of the centre of gravity of three equal particles placed at $A, B, C$ respectively, are

$$
\bar{x}=\frac{1}{8}\left(x_{1}+x_{8}+x_{8}\right) ; \quad \bar{y}=\frac{1}{6}\left(y_{1}+y_{3}+y_{8}\right) ; \quad \bar{z}=\frac{1}{8}\left(z_{1}+z_{2}+z_{3}\right) \text {. }
$$

By what we have just proved, these are also the co-ordinates of the centre of gravity of the triangle $\triangle B C$.

It may be remarked that in Art. 66 the co-ordinates may be rectangular or oblique.

(4) To find the centre of gravity of a pyramid on a triangular base.

Let $A B C$ be the base, $D$ the vertex; bisect $A C$ at $E$; join $B E, D E$; take $E F=\frac{1}{3} E B$, then $F$ is the centre of gravity of $A B C$. Join $F D$; draw $a b, b c$, ca parallel to $A B, B C, C A$

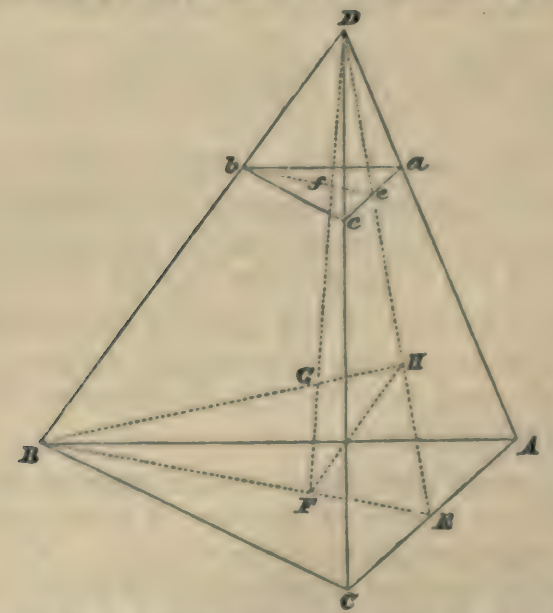

respectively, and let $D F$ meet the plane abc at $f$; join of and produce it to meet $D E$ at $e$. Then, by similar triangles, $a e=e c ;$ also

$$
\frac{b f}{B F}=\frac{D f}{D F}=\frac{e f}{E F} ;
$$

but $E F=\frac{1}{2} B F$, therefore $e f=\frac{1}{2} b f$;

therefore $f$ is the centre of gravity of the triangle $a b c$; and 
if we suppose the pyramid to be made up of an indefinitely great number of indefinitely thin triangular sliesa parallel to the hase, each of these slices lias its centre of gravity in DF. Hence the centre of gravity of the pyramid is in $D F$.

$A$ gain, take $E H=\frac{1}{8} E D$; join $I B B$ cutting $D F$ at $G$. Then, ns hefore, the centre of gravity of the pyramid muat be in BII; but it is in DF; hence $G$, the point of intersection of these straight lines, is the centre of gravity.

Join $F H_{;}$then $F H$ is parallel to $D B$. Also because $E F=+\angle B$, therefore $F H=\frac{1}{d} D B$, and

$\frac{F G}{F \| I}=\frac{D G}{D I} ;$ but $F H=\$ D B$, therefore $F G=\{D G=+D F$.

Hence the centre of gravity is onc-fourth of the way up the straight line joining the centre of gravity of the base with the vertex.

In the same way as the corresponding results were demonstrated for the triangle, we may establish the following:

The centre of gravity of a pyramid coincides with the centre of gravity of particles of equal mass placed at the angular points of the pyramid.

Let $x_{1}, y_{1}, z_{1}$ be the co-ordinates of one angular point; $x_{3}, y_{2}, z$, the co-ordinates of another; and so on; let $\vec{x}, \vec{y}, z$ be the co-ordinates of the centre of gravity of the pyramid: then

$$
\begin{aligned}
& \bar{x}=t\left(x_{1}+x_{1}+x_{3}+x_{2}\right), \\
& \bar{y}=t\left(y_{1}+y_{3}+y_{3}+y_{2}\right), \\
& \bar{z}=t\left(z_{2}+z_{3}+z_{3}+z_{2}\right) .
\end{aligned}
$$

(5) To find the centre of gravity of any pyramid having a plane base.

Divide the base into triangles; if any part of the base is curvilinear then suppose the curve to be dirided into an indefinitely great number of indefinitely short straight linea. Join the vertex of the pyramid with the centres of gravity of all the triangles, and also with all their angles. Draiv a plane parallel to the base at a distance from the base equal to one-fourth of the distance of the vertex from the base; then 
this plane cuts every straight line drawn from the vertex to the base in parts having the same ratio of 3 to 1 ; and therefore the triangular pyramids have their centres of gravity in this plane, and therefore the whole pyramid has its centre of gravity in this plane.

Again, join the vertex with the centre of gravity of the hase; then every section parallel to the base will be similar to the base, and if we suppose the pyramid divided into an indefinitely large number of indefinitely thin slices by planes parallel to the base, the centre of gravity of each slice will lie on the straight line joining the vertex with the centre of gravity of the base. Hence the whole pyramid has its centre of gravity in this straight line.

Therefore the centre of gravity is one-fourth of the way up the straight line joining the centre of gravity of the base with the vertex.

(6) To find the centre of gravity of the frustum of a pyramid furmed by parallel planes.

Let $A B C a b c$ be the frustum; $G, g$ the centres of gravity of the pyramids $D A B C$, Dale; it is clear that the centre of gravity of the frustum must be in $g G$ produced; suppose it at $G^{\prime}$.

$$
\text { Let } F f=c, A B=a, a b=b \text {. }
$$

Since the whole pyramid $D A B C$ is made up of the frustum and the small pyramid, therefore,

$$
\frac{G G^{\prime}}{G g}=\frac{\text { weight of small pyramid }}{\text { weight of frustum }}
$$

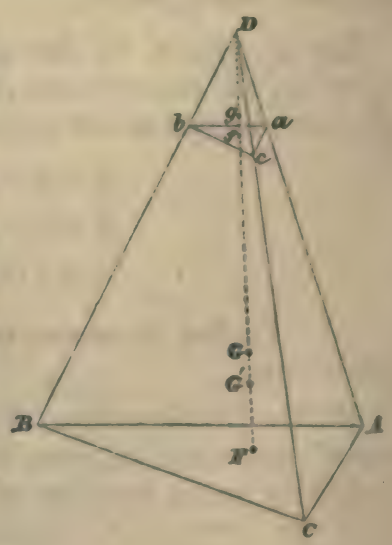

$$
=\frac{\text { vol. of small pyr. }}{\text { vol. of large pyr. }- \text { vol. of small pyr. }}=\frac{l^{3}}{a^{3}-b^{3}},
$$


since similar solita are as the culies of their homologoss alges;

and

$$
\begin{gathered}
C i g=D(i-D g=?(D F-D f)=3 e ; \\
\text { therefore } G G^{\circ}=\frac{80}{4} \cdot \frac{b^{3}}{a^{3}-b^{\circ}}
\end{gathered}
$$

(1): $G F-\frac{1}{2} D F=\frac{1}{b}(D F-D f) \frac{a}{a-b}$ by similar figures,

$$
=\frac{c}{4} \cdot \frac{a}{a-b}
$$

$$
\text { thercfore } \begin{aligned}
F G=F\left(t-G^{\prime} G\right. & =\frac{c}{4}\left\{\frac{a}{a-b}-\frac{3 b^{3}}{a^{3}-b^{2}}\right\} \\
& =\frac{c a^{2}+2 a b+8 b^{3}}{a^{2}+a b+b^{2}}
\end{aligned}
$$

This is true of a fruatum of a pyramid on ary base, $a$ and 7 bitig lomoligious sides of the two ends.

15) We may by the aid of the theory of the centre of gravity domonafrate some geometrical propositions. For example: the struight lises which join the middle points of the opposite alyes of a tetrabalion meet at a point schich biscits each siraighe line.

For suppose equal particles placed at the corners of a tetrahedron; then to find the ecntre of gravify of the system we may procecd tluss: The centre of gravity of any pair of particles is at the middle point of the elige which joins them: and the centre of gravity of the other pair is at the midale point of the opposite edge: then the centre of gravity of the system is at the middle point of the straight line which joins the midile points of the selected eders. And the same point will of course be obtained for the centre of erarity of the system, whaterer pair of edges be selected. Ilence the req̨uired result is obtainerl. 
(8) Particles are placed at the corners of a tetrahertron, the mass of cach particle being proportional to the area of the opposite face: shew that the centre of gravity of the system coincides with the centre of the sphere inscribed in the tetrahedron.

Let $A, B, C, D$ be the angular points of the tetrahedron. Let $p$ be the perpendicular from $D$ on the face $A B C$. Then the distance of the centre of gravity of the system from the plane $A B C$

$$
\begin{aligned}
& =\frac{p \times \text { area of face } A B C}{\text { sum of the areas of the faces }} \\
& =\frac{3 \times \text { volume of tetrahedron }}{\text { sum of the areas of the faces }} .
\end{aligned}
$$

And this expression is equal to the radius of the sphere inscribed in the tetrahedron.

Hence the required result is obtained.

(9) A polyhedron is circumscribed about a sphere; at the points of contact masses are placed which are proportional to the areas of the corresponding faces of the polyhedron: shew that the centre of gravity of these masses coincides with the centre of the sphere.

Take the centre of the sphere for origin, and any plane through the origin for the plane of $(x, y)$.

Let $A_{1}, A_{2}, A_{3}, \ldots$. denote the areas of the faces of the polyhedron; let $z_{1}, z_{2}, z_{s}, \ldots$ denote the ordinates of the points of contact; $\bar{z}$ the ordinate of the centre of gravity. Then, by Art. 66,

$$
\bar{z}=\frac{A_{1} z_{3}+A_{g} z_{3}+A_{8} z_{3}+\ldots}{A_{1}+A_{2}+A_{s}+\ldots} .
$$

Now the projection of the area $A_{1}$ on the plane of $(x, y)$ is $\frac{A_{1} z_{1}}{r_{1}}$, where $r_{3}$ is the radius of the sphere; and similarly for the other projections. And the sum of such projections is zero. Thus $\bar{z}=0$; and since the plane of $(x, y)$ is any plane through the centre of the sphere, the centre of gravity must coincide with the centre of the sphere. 
(10) From amy poine within an equiangular polygon per: pemilivalurs are drucn on all the sides of the polygon, and are produced in a comstant ratio; at the extremitien of the straighe lines thus druirn equal particles are placed: deter. mine the centre of graxity of the system.

1.et e be the distance of the point from which the perpendiculars are drawn from a fixed origin, and a the angle which this distance makes with a fixed straight line which coincides with one of the perpendiculars. Tet $n$ be the number of siles in the polygon, and $y=\frac{2 \pi}{n}$. Iet $p_{-}$denote the perjendicular from the origin on the $m^{\text {ts }}$ side of the polygon; then the correspomling perpendicular from the assumed point is $P_{-}-c \cos (m, \beta-a)$. Let $r$ denote the eunstant ratio. Then if $x_{\infty}$ and $y_{-}$are the co-urdinates of the $m^{\text {क }}$ particle we have

$$
\begin{aligned}
& x_{-}=r\left\{p_{-}-c \cos (m \beta-a)\right\} \cos m \beta+c \cos \alpha_{1} \\
& y_{-}=r\left[p_{-}-c \cos (m \beta-a)\right] \sin m \beta+c \sin \alpha_{0}
\end{aligned}
$$

Hence proceeding as in Proposition rIIr. at the end of Chapter In. we obtain for the co-ordinates of the centre of gravity

$$
\begin{aligned}
& \bar{x}=\xi-\frac{r c}{2} \cos \alpha+c \cos \alpha, \\
& \bar{y}=\eta-\frac{r}{2} \sin \alpha+c \sin \alpha,
\end{aligned}
$$

where $\quad \xi=\frac{r}{n} \Sigma_{p_{\infty}} \cos m \beta, \quad \eta=\frac{r}{n} \Sigma_{P_{\infty}} \sin m \beta$.

Hence if $r=2$ we have

$$
\bar{x}=\xi, \quad \bar{y}=\eta ;
$$

sn that in this case the position of the centre of gravity is independent of the position of the assumed point.

We proceed now to the analytical calculations.

110. In all the cases in which the Integral Calculus is curployed to ascertain the centre of gravity of a body the 
principle is the same; the body is divided into an indefinitely large number of indefinitely small elements; the volume of an element is estimated, and this being multiplied by the density gives the mass of the element. 'The mass is multiplied by the abscissa of the element, and we find the sum of the values of this product for all the elements; the result corresponds to the $\Sigma P x$ of Art. 66. Also we find the sum of the masses of all the elements and thus obtain a result corresponding to the $\Sigma P$ of the same Article. Divide the former result by the latter and we have the value of $\bar{x}$; similarly $y$ and $z$ can be found. In the following cxamples the student must not allow the details of the Integral Calculus to obseure his recognition of the fundamental formula of Art. 66 ; he must consider in every case what corresponds to the $P, x, y, z$ of that Article, that is, he must carefully ascertain into what elements the body is decomposed.

\section{Plane Area.}

111. Iet $C B E H$ be an area bounded by the ordinates $B C$ and $E I I$, the curve $B E$, and the portion $C I I$ of the axis of $x$; it is required to find the centre of gravity of the area. Or instead of the area we may ask for the centre of gravity of a solid bounded by two planes parallel to

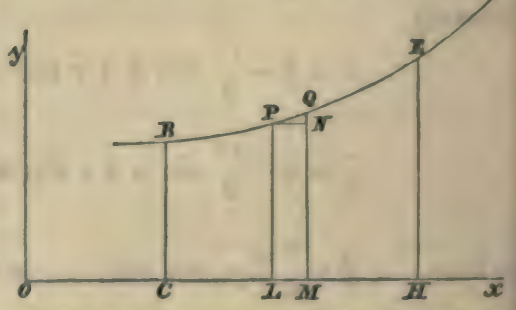
the plane of the paper and equidistant from it, and by a straight line which moves round the boundary CBEH remaining always perpendicular to the plane of the paper. Divide $\mathrm{CH}$ into $n$ portions, and suppose ordinates drawn at the points of division. Let $L P$ and $M Q$ represent two consecutive ordinates, and draw $P N$ parallel to $L M$.

$$
\text { Let } O L=x, L P=y, L M=\Delta x, \quad O C=c, \quad O H=h \text {. }
$$

The area of the rectangle $P M$ is $y \Delta x$; suppose $u$ to denote the area of $P Q N$, and let $x^{\prime}$ be the abscissa of the centre of 
gravity of the area PQML. Then if $k$ denote the thicknesn of the solid and $\rho$ ita denvity. $\operatorname{lo}(y \perp x+u)$ is the mass of the element PQML. Henec, if $x$ be the abscissa of the centre of gravity of the whole figure CBEH, by Art. 66,

$$
\bar{u}=\frac{\Sigma k \rho x^{\prime}(y \Delta x+u)}{\Sigma k p(y \Delta x+u)}=\frac{\Sigma x^{\prime}(y \Delta x+u)}{\Sigma(y \Delta x+u)},
$$

supposing the thickness and density uniform. The summation is to include all the figures like $P Q M$, which are comprised in $C B E H$.

Now suppose $n$ to increaso without limit, and each of the portions $I . I C$ to diminish without limit; then the term $\Sigma_{u}$ in the denominator of $\vec{r}$ vanishes; for it expresses the sum of all the fizures like $P Q N$, and is therefore leas than a reetangle having for its breadth $\Delta x$ and for its height the greatest ordinate comprised between $C B$ and IIE. Also the term $X^{\prime} u$ in the numerator of $\bar{x}$ vanishes, for it is less than the product hIn, and as we have just shewn, this ultimately vanishes. Hence the expression for $\bar{x}$ becomes, when the number of divisions is indefinitely increased and each term indefinitely dimirrished,

$$
\frac{\sum x y \Delta x}{\sum y \Delta x}
$$

Morcover, $x$ must lie between $x$ and $x+\Delta x$ : suppose it equal to $x+v$, where $v$ is less than $\Delta x$; then the numerator of $x$ may be written

\section{$\mathbf{\Sigma} x y \Delta x+\mathbf{\Sigma} v y \Delta x$}

snil as the latter term caunot he so great as $\Delta x \Sigma_{y} \perp x$, it ultimately ranishes. Hence we have

$$
\bar{x}=\frac{\Sigma x y \Delta x}{\Sigma y \Delta x}
$$

that is, the above formula will give the correct ralue of $\bar{x}$ echen oce increase the number of divisions indefinitely and diminish each term indefinitely, aind extend the summation over the spruce CBEII. This will be expressed accorling to the ordinary notation of the Integral Calculus thus,

$$
\bar{x}=\frac{\int_{1}^{x} x y d x}{\int i y d x}
$$


In the same manner we may shew that

$$
\bar{y}=\frac{\int_{e}^{2} y y d x}{\int_{0}^{h} y d x}
$$

where $y$ ' is the limiting value of the ordinate of the centre of gravity of the element $P Q . M L$ when its breadth is indefinitely diminished; $y^{\prime}$ is therefore $=\frac{1}{2} y$; hence

$$
\bar{y}=\frac{\frac{1}{2} \int_{e}^{n} y^{2} d x}{\int_{0}^{n} y d x}
$$

We have now only to substitute in (1) and (2) for $y$ its value in terms of $x$, and then to effect the integration by the ordinary methods.

112. It will not be necessary for the student in solving an example to repeat the whole of the preceding process. When he understands how the necessary exactness may be given, if required, he may proceed shortly thus. The figure $P Q M L=y \Delta x$ ultimately, and the co-ordinates of its centre of gravity are $x$ and $\frac{1}{2} y$ ultimately. Hence

$$
\bar{x}=\frac{\int x y d x}{\int y d x} \text { and } \bar{y}=\frac{\int \frac{1}{2} y y d x}{\int y d x},
$$

the integrations being taken between proper limits.

Unless the contrary be specified, we shall hereafter suppose the bodies we consider to be of uniform density, and shall therefore not introduce any factor to represent the density, because, as in the preceding Article, the factor will disappear.

113. Ex. 1. Let the curve be a parabola whose equation is

$$
\begin{aligned}
& y=2 \sqrt{ }(a x) \text {. } \\
& \text { Here } \bar{x}=\frac{\int_{c}^{n} y x d x}{\int_{0}^{A} y d x}=\frac{\int_{0}^{n} 2 \sqrt{ }(a x) x d x}{\int_{0}^{b} 2 \sqrt{ }(a x) d x}=\frac{\int_{c}^{n} x^{3} d x}{\int_{c}^{n} x^{1} d x} \\
& =\frac{i\left(h^{\mathfrak{l}}-c^{\mathfrak{l}}\right)}{\frac{i}{3}\left(h^{l}-c^{\boldsymbol{l}}\right)} \text {. }
\end{aligned}
$$


If $c=0, \bar{x}=\hat{l}$, which determines the shacisan of the centre of gravity of a portion of a parabolic area beginning at the vertex. Also

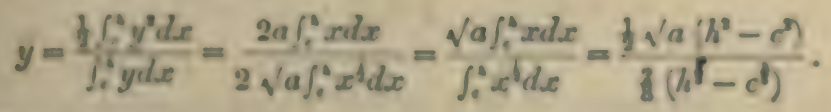

When $\theta=0, \bar{y}=\frac{i}{2} \sqrt{ }(a h)$.

Fix. 2. Iet the curve be an ellipse whose equation is

$$
y=\frac{b}{a} \sqrt{ }\left(a^{2}-2^{2}\right)
$$

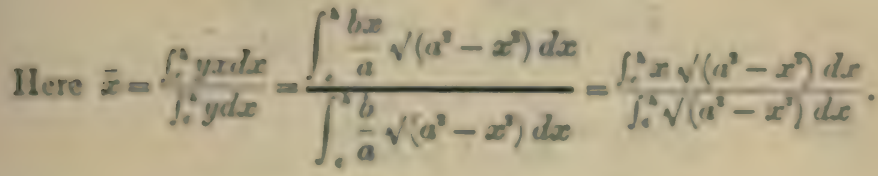

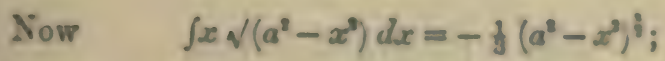

therefure $\int_{0}^{2} x \sqrt{ }\left(a^{2}-x^{2}\right) d x=\frac{1}{3}\left(a^{2}-c^{2}\right)^{1}-\frac{1}{3}\left(a^{2}-h^{2}\right)^{2}$.

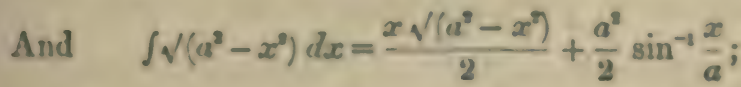

therefore

f. $\sqrt{ }\left(a^{2}-x^{2}\right) d x=\frac{h \sqrt{ }\left(a^{2}-h^{2}\right)-c \sqrt{ }\left(a^{2}-c^{2}\right)}{2}+\frac{a^{2}}{2}\left(\sin ^{-1} \frac{h}{a}-\sin ^{-1} \frac{c}{a}\right)$.

Hence $\bar{x}$ is known.

$$
\begin{aligned}
& \text { Also } \bar{y}=\frac{\int_{0}^{4} \frac{y^{3}}{2} d x}{\int_{0}^{1} y(x)}=\frac{\frac{b}{2 a} \int_{e}^{n}\left(a^{2}-x^{2}\right) d x}{\int_{0}^{1} \sqrt{\left(a^{2}-x^{2}\right) d x}} \\
& =\frac{\frac{b}{2 a}\left\{a^{2}(h-c)-\frac{h^{2}-c^{2}}{3}\right\}}{\frac{\sqrt{\left(a^{2}-h^{2}\right)-c \sqrt{\left(a^{2}-c\right)}}}{2}+\frac{a^{2}}{2}\left(\sin ^{-1} \frac{h}{a}-\sin ^{-1} \frac{c}{a}\right)} \text {. }
\end{aligned}
$$


If we require the centre of gravity of the quadrant of the ellipse, we must put $c=0$ and $h=a$. Hence

$$
\bar{x}=\frac{4 a}{3 \pi}, \quad \bar{y}=\frac{4 b}{3 \pi} .
$$

Ex. 3. Let the curve be a cycloid whose equation is

$$
y=\sqrt{ }\left(2 a x-x^{2}\right)+a \operatorname{vers}^{-1} \frac{x}{a} ;
$$

and suppose we require the centre of gravity of half the area of the curve; then

$$
\bar{x}=\frac{\int_{0}^{2 a} y x d x}{\int_{0}^{2 a} y d x}, \quad \bar{y}=\frac{\frac{1}{2} \int_{0}^{2 a} y^{2} d x}{\int_{0}^{2 a} y d x} .
$$

Now

$$
\begin{aligned}
\int y x d x & =\frac{y x^{2}}{2}-\int \frac{x^{2}}{2} \frac{d y}{d x} d x \\
& \left.=\frac{y x^{2}}{2}-\int \frac{x^{2}}{2} \sqrt{\left(\frac{2 a-x}{x}\right)}\right) d x .
\end{aligned}
$$

Also, when $x=0, y=0$, and when $x=2 a, y=\pi a$;

therefore $\int_{0}^{2 a} y x d x=\frac{1}{2}\left\{\pi a \cdot(2 a)^{3}\right\}-\frac{1}{2} \int_{0}^{2 a} x \sqrt{ }\left(2 a x-x^{2}\right) d x$ :

and as $\int_{0}^{2 a} x \sqrt{ }\left(2 a x-x^{2}\right) d x$ will be found $=\frac{1}{2} \pi a^{3}$, we have

$$
\int_{0}^{2 a} y x d x=2 \pi a^{3}-\frac{1}{4} \pi a^{8}=\frac{7}{4} \pi a^{8} \text {. }
$$

Again,

$$
\begin{aligned}
\int y d x & =y x-\int x \frac{d y}{d x} d x \\
& =y x-\int \sqrt{ }\left(2 a x-x^{2}\right) d x ;
\end{aligned}
$$

therefore

$$
\begin{aligned}
\int_{0}^{2 a} y d x & =2 \pi a^{2}-\int_{0}^{2 a} \sqrt{ }\left(2 a x-x^{2}\right) d x \\
& =2 \pi a^{2}-\frac{1}{2} \pi a^{2}=\frac{3}{2} \pi a^{2} ; \\
\bar{x} & =\frac{\frac{7}{4} \pi a^{3}}{\frac{3}{2} \pi a^{3}}=\frac{7}{6} a .
\end{aligned}
$$

therefore 
Also

$\int y^{2} d x=y^{2} x-2 \int y x \frac{d y}{d x} d x$

$$
\begin{aligned}
& =y^{2} \cdot x-2 \int y \sqrt{ }\left(2 a x-x^{2}\right) d x \\
& =y^{2} x-2 \int\left(2 a x-x^{2}\right) d x-2 a \int \sqrt{ }\left(2 a x-x^{3}\right) \operatorname{vers}^{-1} \frac{x}{a} d x \\
& =y^{2} x-2 a x^{3}+\frac{2 x^{2}}{3}-2 a \int \sqrt{ }\left(2 a x-x^{2}\right) v^{-1} \frac{x}{a} d x
\end{aligned}
$$

therefore $\int_{0}^{\infty} y^{2} d x=2 \pi^{2} a^{3}-\frac{8 a^{3}}{3}-2 a \int_{0}^{\infty} \sqrt{ }\left(2 a x-x^{2}\right) \operatorname{vers}^{-1} \frac{x}{a} d x$.

By assuming vers ${ }^{-1} \frac{x}{a}=\theta$, we may shew that

$$
\int_{0}^{\infty} \sqrt{\left(2 a x-x^{2}\right) \operatorname{vers}^{-1} \frac{x}{a} d x=\frac{\pi^{2} a^{2}}{4}} \text {. }
$$

Ience

$$
\int_{0}^{2} y^{3} d x-3 \pi^{3} a^{2}-a^{3}
$$

therefore

$$
\bar{y}=\frac{\frac{1}{2} a\left(\frac{3}{2} \pi^{2}-\pi\right)}{\frac{1}{3} \pi}=\frac{a}{3 \pi}\left(\frac{3}{2} \pi^{2}-3\right) \text {. }
$$

114. If a curve have a branch below the axis of $x \mathrm{sym}$ metrical with one above the axis, and we require the centre of gravity of the area bounded by the two branches and ordinates drawn at the distances $c$ and $h$ from the origin, we havo

$$
\bar{x}=\frac{2 \int_{0}^{2} y x d x}{2 \int_{0}^{2} y d x}-\int_{0}^{2} y x x d x
$$

and

$$
\bar{y}=0 \text {. }
$$

115. Wo have hitherto supposed the axes roctangular; if they are oblique and inclined at an angle $\omega$, then the figure PQ.ML (see fig. to $A \mathrm{rt}$. 111) will $=\sin \omega y \Delta x$ ultimately. Ilence the formula (1) and (2) of Art. 111 remain true, for sin $\omega$ oceurs as a factor in tho numerator and denominator, and may therefore bo cancelled. 
116. It is sometimes convenient to use polar formulx.

Let $D E$ be the arc of a curve; and suppose we require the centre of gravity of the area comprised between the are $D E$ and the radii $O D, O E$ drawn from the pole $O$.

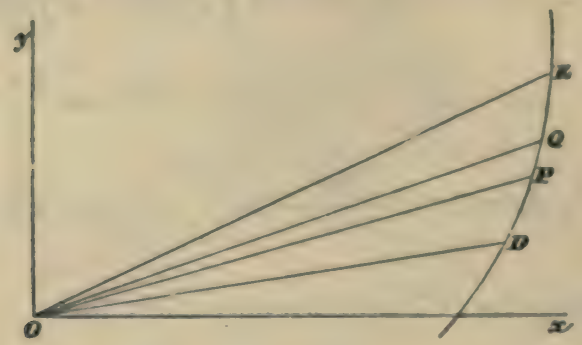

Divide the angle $D O E$ into a number of angles, of which $P O Q$ represents one; let $O P=r, P O x=\theta, P O Q=\Delta \theta$. The area $P O Q=\frac{1}{2} r^{2} \Delta \theta$ ultimately (Diff. Calc., Art. 313). Also the centre of gravity of the figure $P O Q$ will be ultimately, like that of a triangle, on a straight line drawn from $O$ bisecting the chord $P Q$, and at a distance of two-thirds of this straight line from $O$. Hence the abscissa and ordinate of the centre of gravity of $P O Q$ will be ultimately

$$
\frac{2}{2} r \cos \theta \text {, and } \frac{2}{3} r \sin \theta \text { respectively. }
$$

Hence

$$
\begin{aligned}
& \bar{x}=\frac{\int \frac{2}{2} r \cos \theta \frac{1}{2} r^{2} d \theta}{\int \frac{1}{2} r^{2} d \theta}=\frac{\frac{2}{3} \int r^{2} \cos \theta d \theta}{\int r^{2} d \theta}, \\
& \bar{y}=\frac{\int \frac{2}{3} r \sin \theta \frac{1}{2} r^{2} d \theta}{\int \frac{1}{2} r^{2} d \theta}=\frac{\frac{2}{2} \int r^{2} \sin \theta d \theta}{\int r^{2} d \theta} .
\end{aligned}
$$

In these formulæ we must put for $r$ its value in terms of $\theta$ given by the equation to the curve; we must then integrate from $\theta=\alpha$ to $\theta=\beta$, supposing $\alpha$ and $\beta$ the angles which $O D$ and $O E$ respectively make with the fixed straight line $O x$. 
117. Fix. Iet $O$ be the fincus of $n$ parabola, and the fixed straight line $O x$ pass through the vertes; then

$$
r=\frac{a}{\cos ^{2} t \theta},
$$

where $t a$ is the latus rectum of the parabola.

Henco

$$
\bar{x}=\frac{3 a \int_{0}^{\theta} \frac{\cos \theta}{\cos ^{2} \theta \theta} d \theta}{\int_{0}^{\beta} \frac{d \theta}{\cos ^{2} t \theta}} .
$$

$$
\begin{aligned}
& \text { Now } \int \frac{\cos \theta}{\cos ^{2} \frac{1}{2} \theta} d \theta=\int \frac{\cos ^{2} \int \theta-\sin ^{2} \frac{1}{2} \theta}{\cos ^{2} \frac{1}{2} \theta} \sec ^{2} \frac{1}{2} \theta d \theta \\
& =\int\left(1-\tan ^{2} \frac{1}{2} \theta\right)\left(1+\tan ^{2} \frac{1}{2} \theta\right) \sec ^{2} \frac{1}{2} \theta \cdot d \theta \\
& =\int\left(1-\tan ^{6} \frac{1}{2} \theta\right) \sec ^{2} \frac{1}{d} \theta \cdot 1 \theta=2\left(\tan \frac{1}{d} \theta-\frac{1}{b} \tan ^{6} \frac{1}{2} \theta\right) \text {; }
\end{aligned}
$$

therefore $\int_{0}^{\beta} \frac{\cos \theta}{\cos ^{2} \frac{2}{2} \theta^{2}} d \theta=2\left(\tan \frac{3}{2} \beta-\tan \frac{1}{2} \alpha\right)-\frac{2}{3}\left(\tan ^{2} \frac{1}{2} \beta-\tan ^{2} \frac{1}{2} \alpha\right)$.

$$
\text { Also } \int \frac{d \theta}{\cos ^{0} \frac{1}{2} \theta}=\int\left(1+\tan ^{2} \frac{1}{2} \theta\right) \sec ^{2} \frac{1}{2} \theta d \theta=2 \tan \frac{1}{2} \theta+i \tan ^{2} \frac{1}{2} \theta \text {; }
$$

therefore $\int_{-}^{\beta} \frac{d \theta}{\cos ^{\circ} \frac{1}{2} \theta}=2\left(\tan \frac{1}{2} \beta-\tan \frac{1}{2} \alpha\right)+\frac{\gamma}{2}\left(\tan ^{\circ} \frac{1}{2} \beta-\tan ^{2} \frac{1}{2} \alpha\right)$ :

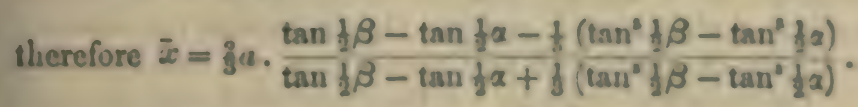

Again, $\int \frac{\sin \theta}{\cos ^{2} \frac{1}{2} \theta} d \theta=2 \int \frac{\sin \frac{1}{2} \theta}{\cos ^{2} d \theta} d \theta=\frac{1}{\cos ^{\circ} d \theta^{\theta}}$;

therefore $\quad \int_{-\alpha}^{\beta} \frac{\sin \theta}{\cos ^{6} \frac{1}{2} \theta} d \theta=\sec ^{\circ} \frac{1}{2} \beta-\sec ^{6} \frac{1}{2} \alpha$;

thereforo $\bar{y}=f a \cdot \frac{\sec ^{4} \frac{1}{2} \beta-\sec ^{4} \frac{1}{d} \alpha}{\tan \frac{1}{d} \beta-\tan \frac{1}{2} \alpha+\frac{1}{b}\left(\tan ^{2} \frac{1}{2} \beta-\tan ^{\circ} \frac{1}{2} \alpha\right)}$. 


\section{Plane Area. Double Integration.}

118. There is another method of dividing a plane area into elements, to which we now proceed.

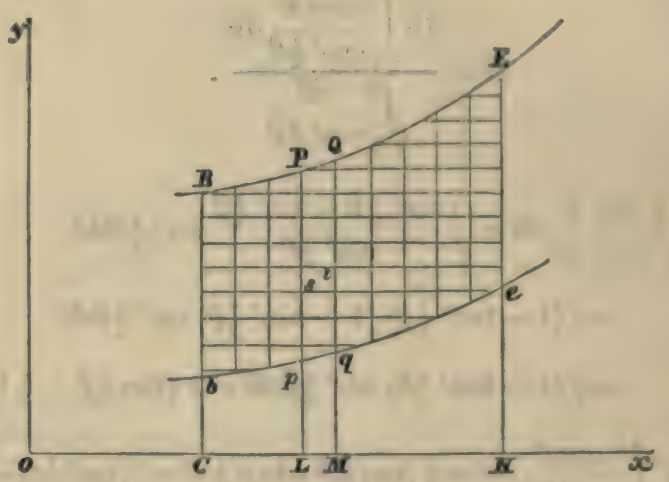

Let a series of straight lines be drawn parallel to the axis of $y$, and another series of straight lines parallel to the axis of $x$. Let $s t$ represent one of the rectangles formed by these straight lines; and suppose $x$ and $y$ to be the co-ordinates of 8 , and $x+\Delta x$ and $y+\Delta y$ the co-ordinates of $t$. 'T'hen the area of the rectangle st is $\Delta x \Delta y$, and the co-ordinates of its centre of gravity are ultimately $x$ and $y$. Hence, to find the abscissa of the centre of gravity of any plane area, we can take the sum of the values of $x \Delta x \Delta y$ for the numerator, and the sum of the values of $\Delta x \Delta y$ for the denominator, $\Delta x$ and $\Delta y$ being indefinitely diminished. This is expressed thus,

$$
\bar{x}=\frac{\iint x d x d y}{\iint d x d y} .
$$

Similarly,

$$
\bar{y}=\frac{\iint y d x d y}{\iint d x d y}
$$


119. Suppose, for example, thint the ares in bounded by the two ordinates $B b C, E I I$, and the two curves $B P Q E$, byge. Let $y=\phi(x)$ be the equation to the upper curve, and $y=\psi(x)$ the equation to the lower curve; let $O C=c$, $0 \| 1=h$. The sum of the product $x \Delta x \Delta y$ for all the rectangles similar to $s t$, which are contained in the strip $P Q q p$, is squal to is $\Delta x$ multiplied by the sum of the values of $\Delta y$, for $x \Delta x$ hins the same value for each of these rectangles. Since the sum of the values of $\Delta y$ is $I_{p}$ or $\phi(x)-\psi(x)$, we have $x \Delta x \cdot\{\phi(x)-\psi(x))$ as the result obtained by considering all the rectangles in the strip PQgP. We have then to sam up the values of $x \Delta x|\phi(x)-\psi(x)|$ for all the strips similar to

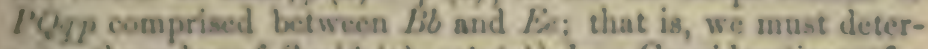
mine the value of $f x|\phi(x)-\psi(x)| d x$. Considerations of a similar kind apply to the denominator of $x$, and we obtain

$$
\bar{x}=\frac{\int_{0}^{4} x(\phi(x)-\psi(x)-\psi(x)] d x}{\int(x)} .
$$

In the numerator of $\bar{y}$ we observe that $y \Delta y \Delta x$ represents that fortion of it which arises from the element $s t$; hence we shall find the result obtained from all the elements in the strip PQAP, if we determine the sum of all the ralues of $y \Delta y$. and muluply the result by $\Delta x$. Now the sum of the ralues of $y \Delta y$ is $\int_{\beta \rightarrow \infty}^{\phi} y d y$, or $d\left[\{\phi(x)\}^{\prime}-\left\{\left.\psi(x)\right|^{2}\right]\right.$. If we multiply by $\Delta x$, and find the sum of the values of the prodnct for all the strips between $B b$ and $F_{e}$, we obtain the numerator of $y$. Hence

$$
\bar{y}=\frac{d \int:\left[\left.\phi(x)\right|^{2}-\{\psi(x)]^{2}\right] d x}{\int:(\phi(x)-\psi(x)\} d x} .
$$

The value of $\bar{y}$ may be written thus

$$
\bar{y}=\frac{\int_{0}^{n} f\{\phi(x)+\psi(x)\}[\phi(x)-\psi(x)] d x}{\int_{0}^{H}\left\{\phi(x)-\psi(x) \int d x\right.} .
$$

The meaning of the factors in the numerator is now spparent; for $\{\phi(x)-\psi(x)\} \Delta x$ ultimately represents the area of the strip $P Q q P$, and $\frac{1}{2}[\phi(x)+\psi(x)]$, which is the ordinate of the midule point of $Y_{P}$, will ultimately be the ordinate 
of the centre of gravity of $P Q q p$. Hence the above equation agrees with that given in $A$ rt. 66 ,

$$
\bar{y}=\frac{\Sigma P_{y}}{\Sigma P} .
$$

The process and the figure in the preceding two Articles would have been unnecessary if our only object had been to establish the formula for $\bar{x}$ and $\bar{y}$, since these formulæ can be obtained more simply as we have just shewn. But we shall require hereafter other formulæ involving double integration, and have therefore directed the reader's attention to these in order to accustom him to the subject.

120. Ex. Let $O P E$ be a parabola having for its equation $y^{2}=4 a x$, and $O E$ a straight line having for its equation $y=k x$; find the centre of gravity of the area $O P E$ between the curve and the straight line.

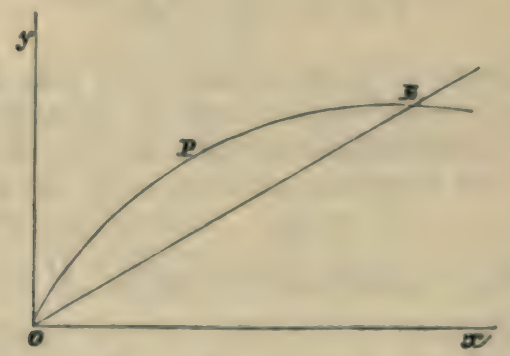

Here $\phi(x)=2 \sqrt{ }(a x), \psi(x)=k x, c=0 ; h$ is to be found from the equation $2 \sqrt{ }(a h)=k h$;

therefore

$$
h=\frac{4 a}{k^{2}} \text {. }
$$

Thus $\bar{x}=\frac{\int_{0}^{h} x\{2 \sqrt{ }(a x)-k x\} d x}{\int_{0}^{h}\{2 \sqrt{ }(a x)-k x\}}$

$$
\begin{aligned}
& =\frac{\frac{5}{5} \sqrt{ } a h^{5}-\frac{1}{8} k h^{8}}{\frac{4}{3} \sqrt{ } a h^{1}-\frac{1}{2} k \cdot h^{2}}=h \frac{\frac{1}{6} \sqrt{ } a-\frac{1}{3} k \sqrt{ } h}{\frac{4}{3} \sqrt{ } a-\frac{1}{2} k \sqrt{ } h}=h \frac{\frac{4}{3}-\frac{2}{3}}{\frac{4}{3}-1} \\
& =\frac{2 h}{5}=\frac{8 a}{5 k^{3}} .
\end{aligned}
$$


Similarly, $\bar{y}=\int_{0}^{1}(2) \int\left(a x-k^{2} x^{2}\right) d x$

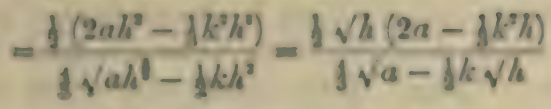

$$
\begin{aligned}
& -\frac{1 \sqrt{k}(2 a-1 a)}{f \sqrt{ } a-\sqrt{a}}=\sqrt{ }(a / h)=\frac{2 a}{k} \text {. }
\end{aligned}
$$

121. Sometimes it will be more convenient to integrate the formular in Art. 118, first with respect to $x$ and then with respect to $\mathrm{y}$. For example, if the given area is comprised between the straight lines $y=c$, and $y=h$, and the curves $x=\psi(y)$, and $x=\phi(y)$, we obtain

$$
\begin{aligned}
& x=\frac{1 F\left[(\phi(y))^{2}-(\psi(y))\right.}{\int f(\phi(y)-\psi(y)) d y},
\end{aligned}
$$

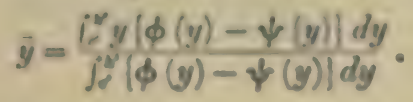

If we apply these to the example given in Art. 120, we have $\downarrow(y)=\frac{y}{t a}, \phi(y)=\frac{y}{k}, c^{\prime}=0$, and $k^{\prime}$ is to be found from the iquation $\frac{h^{\prime}}{k}=\frac{h^{2}}{4 a} ;$ therefore $h^{\prime}=\frac{4 a}{k}$.

IIence

$$
\begin{gathered}
\bar{x}=\frac{\frac{1}{2} \int_{0}^{\infty}\left(\frac{y^{2}}{k^{3}}-\frac{y^{4}}{16 a}\right) d y}{\int_{0}^{\infty}\left(\frac{y}{y^{3}}-\frac{y^{3}}{4 a}\right) d y}, \\
\bar{y}=\frac{\int_{0}^{\infty} y\left(\frac{y}{k}-\frac{y^{2}}{4 a}\right) d y}{\int_{0}^{4}\left(\frac{y}{k^{2}}-\frac{y^{2}}{4 a}\right) d y}
\end{gathered}
$$

The results will of course be the same as before.

For fuller explanations and illustrations of double integrations the student is referred to treatises on the Integral Calcnlus. (See especially Integral Caliculus, Art. 141 and Art. 152.) 
122. We will now give polar furmula involving double integration.

Let a series of straight lines be drawn from a pole $O$, also a series of circles be described from $O$ as a centre. Let $s t$ be one of the elements formed by this mode of dividing a plane area; let $r$ and $\theta$ be the polar co-ordinates of $s, r+\Delta r$ and $\theta+\Delta \theta$ the co-ordinates of $t$; then the area of the element $s t$ will be ulti-

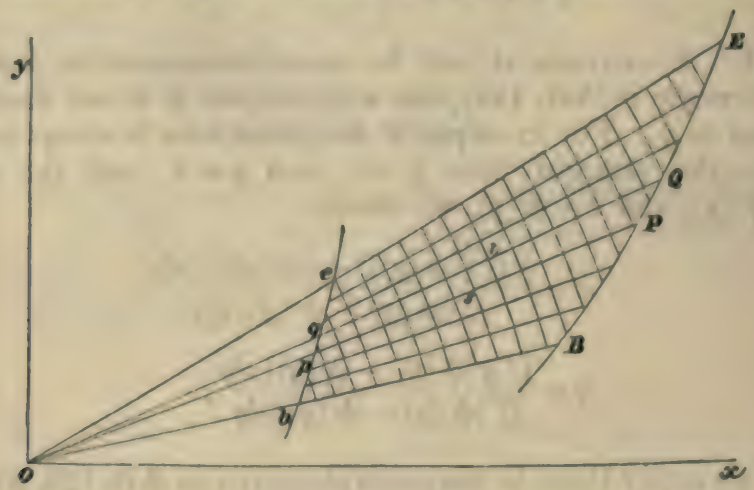

mately $r \Delta \theta \Delta r$, and the abscissa and ordinate of its centre of gravity will be $r \cos \theta$ and $r \sin \theta$ respectively. Hence we obtain

$$
\bar{x}=\frac{\iint r \cos \theta r d \theta d r}{\iint r d \theta d r}=\frac{\iint r^{2} \cos \theta d \theta d r}{\iint r d \theta d r} .
$$

Similarly $\bar{y}=\frac{\iint r^{2} \sin \theta d \theta d r}{\iint r d \theta d r}$.

Suppose the area bounded by the curves $B P Q E$, bpqe, and the radii $O b B, O e E$. Let $r=\phi(\theta)$ be the equation to the first curve, $r=\psi(\theta)$ that to the second; and let $\alpha$ and $\beta$ be the angles which $O B$ and $O E$ make respectively with $O x$.

The sum of the values of $r^{2} \cos \theta \Delta r \Delta \theta$ for all the elements comprised in the strip $P Q_{q p}$, will be found by multiplying the sum of the values of $r^{2} \Delta r$ by $\cos \theta \Delta \theta$; the former sum is ultimately

$$
\int_{\psi(\theta)}^{\phi(\theta)} r^{2} d r \text { or } \frac{1}{\delta}\left[\{\phi(\theta)\}^{3}-\{\psi(\theta)\}^{0}\right]
$$




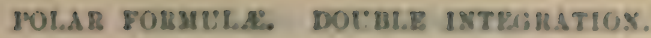

Hence the numerator of the value of $\bar{x}$ is

$$
d j_{0} \cos \theta\left[(\phi(\theta)]^{\circ}-\left\{\left.\psi(\theta)\right|^{\nu}\right] d \theta,\right.
$$

and the denominator, in like manner, is

$$
\text { 1): }\left[\left.\phi(\theta)\right|^{0}-|\psi(\theta)|^{\eta}\right] d \theta \text {; }
$$

therefore $\quad \bar{x}=1 \int^{\pi} \cos \theta\left[\left.i \phi(\theta)\right|^{\circ}-|\psi(\theta)|^{\circ}\right] d \theta$.

$$
\left.\left.j ![\phi(\theta)\}^{3}-\mid \psi(\theta)\right]^{\prime}\right] d \theta
$$

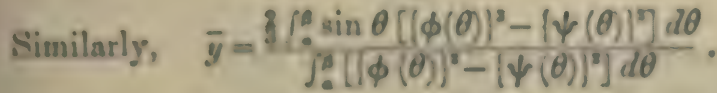

123. Ex. 1. Find the centre of gravity of the area comprised between two semicircles $O p b$ and $O P B$.

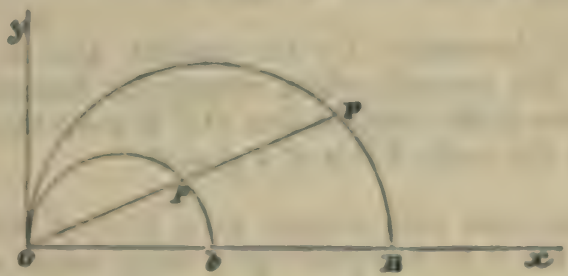

Let $O b=c, O B=h ; \phi(\theta)=h \cos \theta, \psi(\theta)=c \cos \theta ; \alpha=0$, $\beta=\frac{1}{2} \pi ;$ thus

$$
\begin{aligned}
& \bar{x}=\frac{\frac{9}{3}\left(h^{2}-c^{2}\right) \int_{0}^{t+} \cos ^{4} \theta d \theta}{\left(h^{2}-c^{2}\right) \int_{0}^{t+} \cos ^{2} \theta d \theta} \\
& =\frac{2}{3} \cdot \frac{3}{4} \cdot \frac{h^{3}-c^{8}}{h^{2}-c^{2}} \\
& =\frac{1}{2} \frac{h^{0}+h c+c^{\prime}}{h+c} \text {. }
\end{aligned}
$$

(Sce Integ. Cale., Art. 35).

Also

$$
\begin{aligned}
& \bar{y}=\frac{\left(h^{3}-c^{2}\right) \int_{0}^{t-} \sin \theta \cos ^{\theta} \theta d \theta}{\left(h^{3}-c^{2}\right) \int_{0}^{l-} \cos ^{3} \theta d \theta}
\end{aligned}
$$

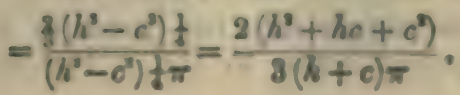


Ex. 2. The sector of a circle.

Let $B O E$ be the sector, subtending an angle $\beta, O B=a$.

In this example we may with equal facility integrate first with respect to $\theta$ and then with respect to $r$, or first with respect to $r$ and then with respect to $\theta$.

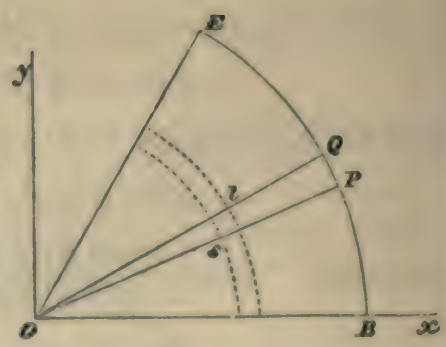

$$
\begin{aligned}
& \bar{x}=\frac{\int_{0}^{a} \int_{0}^{\beta} r^{2} \cos \theta d r d \theta}{\int_{0}^{a} \int_{0}^{\beta} r d r d \theta}=\frac{\sin \beta \int_{0}^{a} r^{2} d r}{\beta \int_{0}^{a} r d r}=\frac{2 a \sin \beta}{3 \beta}, \\
& \bar{y}=\frac{\int_{0}^{a} \int_{0}^{\beta} r^{2} \sin \theta d r d \theta}{\int_{0}^{a} \int_{0}^{\beta} r d r d \theta}=\frac{(1-\cos \beta) \int_{0}^{a} r^{2} d r}{\beta \int_{0}^{a} r d r}=\frac{2 a(1-\cos \beta)}{3 \beta} .
\end{aligned}
$$

It will be instructive for the student also to notice the solution of this example when rectangular formulæ are used. The equation to the straight line $O E$ is $y=x \tan \beta$; and the equation to the circle $E B$ is $x^{2}+y^{2}=a^{2}$.

If we integrate with respect to $x$ first we must integrate from $x=y \cot \beta$ to $x=\sqrt{ }\left(a^{2}-y^{2}\right)$; since when we integrate with respect to $x$ we have to collect all the elements in a strip which is parallel to the axis of $x$, and is bounded by $O E$ at one end and by $E B$ at the other. These strips extend from the axis of $x$ up to $E$, and the ordinate of $E$ is $a \sin \beta$. Hence we integrate with respect to $y$ from $y=0$ to $y=a \sin \beta$. Therefore

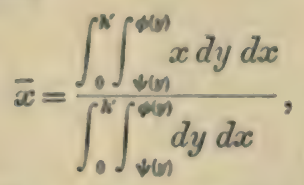

$$
\bar{y}=\frac{\int_{0}^{x} \int_{\nu(x)}^{\phi(x)} y d y d x}{\int_{0}^{\Lambda} \int_{\nu(y)}^{\phi(x)} d y d x},
$$

where $\psi(y)=y \cot \beta, \phi(y)=\sqrt{ }\left(a^{2}-y^{2}\right), h^{\prime}=a \sin \beta$.

\section{The integrations may be easily effected.}

If we wish to integtate with respect to $y$ first, we shall have to divide the figure into two parts by a straight line drawn from $E$ perpendicular to $O B$. For the part to the 
Ieft of the dividing line the limits of $y$ are 0 and $x \tan \beta$, and thise of $x$ are 0 and acos $\beta$. For the part to the right of the divinling line the limits of $y$ are 0 and $\sqrt{ }\left(a^{2}-x^{2}\right)$, and those of $x$ are $a \cos \beta$ and $a$. Hence

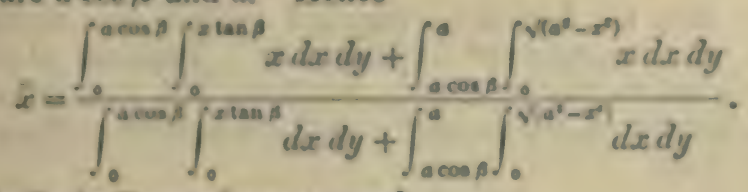

Similarly $\bar{y}$ may be expressed.

We have treated this example as an illustration of integrntion rather than for the purpose of obtaining the result in the nimplest form. We might proced thus; the centre of gravity mast lie on the straight line which hisects the angle EOB. Jleser taking this straiglit line for the initial line and using polat er-ordinates, we hare $\bar{y}=0$, and

$$
\bar{x}=\frac{\int_{0}^{\alpha} \int_{-1}^{1 / \alpha} r^{2} \cos \theta d r d \theta}{\int_{0}^{\infty} \int_{-\alpha}^{\sqrt{\beta}} r d r d \theta}=\frac{4 a \sin 1 \beta}{3 \beta} .
$$

Solid of Revolution.

128. Let a solid be generated by the revolution of the curre $B P Q E$ round the axis of $x$, and suppose we require the centre of grarity of a portion of it intercepted between planes peryendicular to the axis of revolution.

Lat the co-ordinates of s point $P$ in the curre be

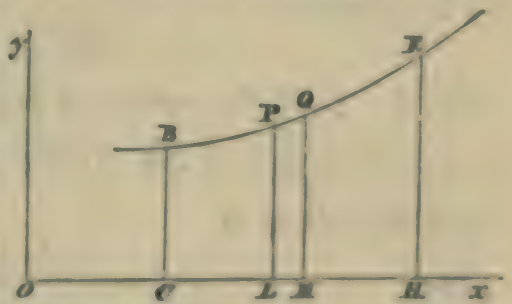

$x$ and $y$, and $x+\Delta x$ the alseissa of an adjacent point $Q$. As the curve revolres round the axis of $x$, the area FQMII. will generate a volume which is ultimately equal to $\pi y^{\prime} \Delta x$. Also the abscissa of its centre of gravity will be $x$ ultimately. Hence

$$
\bar{x}=\int \frac{\pi y^{2} x d x}{\int \pi y^{3} d x}=\frac{\left.\int r^{2} x\right] x}{\int y^{2} d x} .
$$


The centre of gravity of the solid is obviously in the straight line $O x$, so that we only require the value of $\bar{x}$ in order to determine its position.

125. Ex. 1. Let it be required to find the centre of gravity of a portion of a paraboloid. Suppose $y^{2}=4 a x$ the equation to the generating parabola, and that the solid is bounded by planes distant $c$ and $h$ respectively from the vertex; then

$$
\bar{x}=\frac{\int_{c}^{1} 4 a x^{2} d x}{\int_{0}^{4} 4 a x d x}=\frac{2}{3} \cdot \frac{h^{3}-c^{8}}{h^{2}-c^{3}} .
$$

If we put $c=0$ we find for the centre of gravity of a serment of a paraboloid commencing at the vertex

$$
\bar{x}=\frac{2 h}{3} \text {. }
$$

Ex. 2. Required the centre of gravity of a portion of a sphere intercepted between two parallel planes.

- Let $y^{2}=a^{2}-x^{2}$ be the equation to the gencrating circle;

$$
\bar{x}=\frac{\int_{c}^{n}\left(a^{2}-x^{2}\right) x d x}{\int_{c}^{1}\left(a^{2}-x^{2}\right) d x}=\frac{\frac{1}{2} a^{2}\left(h^{2}-c^{2}\right)-\frac{1}{4}\left(h^{4}-c^{6}\right)}{a^{2}(h-c)-\frac{1}{3}\left(h^{2}-c^{3}\right)} .
$$

If we put $c=0$ and $h=a$, we find for the centre of gravity of a hemisphere

$$
\bar{x}=\frac{3}{8} a \text {. }
$$

Ex. 3. Find the centre of gravity of the solid generated by the revolution of the cycloid $y=\sqrt{ }\left(2 a x-x^{2}\right)+a \operatorname{vers}^{-1} \frac{x}{a}$ round the axis of $x$.

Here

$$
\bar{x}=\frac{\int_{0}^{2 a} y^{2} x d x}{\int_{0}^{2 u} y^{2} d x} .
$$

Now $y^{2}=2 a x-x^{2}+2 a \sqrt{ }\left(2 a x-x^{2}\right) \operatorname{vers}^{-1} \frac{x}{a}+a^{2}\left(\operatorname{vers}^{-1} \frac{x}{a}\right)^{2}$.

Thus the numerator of $\bar{x}$ consistz of three integrals of which we will give the values; these values may be obtained without difficulty by transforming the integrals where $\operatorname{vers}^{-1} \frac{x}{a}$ 
occurs by the aasumption vers $\cos ^{-1} \frac{x}{a}=\theta$, so that $x=a(1-\cos \theta$, aud then integrating by parts. We shall find

$$
\begin{aligned}
& \int_{0}^{\infty}\left(2 a x-x^{2}\right) x d x=\frac{4 a^{4}}{3}, \\
& 2 a \int_{0}^{\infty} x \sqrt{\left(2 a x-x^{5}\right) \operatorname{vers}^{-1} \frac{x}{a}} d x=2 a\left(\frac{4 a^{3}}{9}+\frac{\pi^{2} a^{2}}{4}\right) \text {, } \\
& a^{2} \int_{0}^{\infty} x\left(\operatorname{vers}^{-1} \frac{x}{a}\right)^{2} d x=\left(\frac{5 \pi^{2}}{4}-4\right) a^{2} \text {. }
\end{aligned}
$$

Hence the numerator of $\bar{x}$ is $\left(\frac{7 \pi^{2}}{4}-\frac{16}{9}\right) a^{4}$.

Also the denominntor of $\bar{x}$ consists of three integrals which have the followiog values,

$$
\begin{gathered}
\int_{0}^{\infty}\left(2 a x-x^{2}\right) d x=\frac{4 a^{3}}{3}, \\
2 a \int_{0}^{\infty} \sqrt{ } \sqrt{\left(2 a x-x^{2}\right) \operatorname{vers}^{-1} \frac{x}{a} d x=2 a \frac{\pi^{2} a^{2}}{4},} \\
a^{2} \int_{0}^{\infty}\left(\operatorname{vers}^{-1} \frac{x}{a}\right)^{2} d x=\left(\pi^{2}-4\right) a^{3} .
\end{gathered}
$$

Hence the denominator of $\bar{x}$ is $\left(\frac{3 \pi^{3}}{2}-\frac{8}{3}\right) a^{3}$.

Therefore $\quad \bar{x}=\frac{\left(\frac{7 \pi^{2}}{4}-\frac{16}{9}\right) a^{6}}{\left(\frac{3 \pi^{3}}{2}-\frac{8}{3}\right) a^{3}}=\frac{\left(63 \pi^{2}-64\right) a}{6\left(9 \pi^{3}-16\right)}$.

126. If a solid of revolution be formed by revolving a curve round the axis of $y$, we find for the position of the ceutre of gravity

$$
\bar{y}=\frac{\int \pi x^{2} y d y}{\int \pi x^{2} d y}=\frac{\int x^{2} y d y}{\int x^{2} d y} .
$$

For example, let the cycloid $y=\sqrt{ }\left(2 a x-x^{2}\right)+a \operatorname{rers}^{-1} \frac{x}{a}$, revolve round the axis of $y$, and suppose that we require the 
centre of gravity of the volume generated by that half of the curve for which $y$ is positive. Here

$$
\bar{y}=\frac{\int_{0}^{m a} x^{2} y d y}{\int_{0}^{n a} x^{2} d y} .
$$

Now $\int x^{2} y d y=\int x^{2} y \frac{d y}{d x} d x$; thus in the present case,

$$
\int_{0}^{m a} x^{2} y d y=\int_{0}^{2 a} x^{2} y \frac{d y}{d x} d x
$$

Similarly

$$
\int_{0}^{\pi a} x^{2} d y=\int_{0}^{2 a} x^{2} \frac{d y}{d x} d x
$$

Thus $\bar{y}=\frac{\int_{0}^{2 a} x^{2} y\left(\frac{2 a-x}{x}\right)^{d} d x}{\int_{0}^{2 a} x^{2}\left(\frac{2 a-x}{x}\right)^{\frac{1}{2}} d x}=\frac{\int_{0}^{2 a} y x \sqrt{ }\left(2 a x-x^{2}\right) d x}{\int_{0}^{2 a} x \sqrt{ }\left(2 a x-x^{2}\right) d x}$.

The numerator of $y$ consists of two integrals which have the following values,

$$
\begin{aligned}
\int_{0}^{2 a} x\left(2 a x-x^{2}\right) d x & =\frac{4 a^{4}}{3}, \\
a \int_{0}^{2 a} x \sqrt{ }\left(2 a x-x^{2}\right) \operatorname{vers}^{-1} \frac{x}{a} & d x=a\left(\frac{4 a^{8}}{9}+\frac{\pi^{2} a^{3}}{4}\right) .
\end{aligned}
$$

The value of the denominator of $\bar{y}$ is $\frac{\pi}{2} a^{3}$.

$$
\text { Thercfore } \quad \bar{y}=\frac{\frac{4 a^{4}}{3}+\frac{4 a^{4}}{9}+\frac{\pi^{2} a^{4}}{4}}{\frac{\pi}{2} a^{2}}=\left(\frac{16}{9}+\frac{\pi^{2}}{4}\right) \frac{2 a}{\pi} \text {. }
$$

127. We may also find it convenient in some cases to use formulæ involving double integration.

Suppose the figure in Art. 118 to revolve round the axis of $x$; let $x, y$ be the co-ordinates of $s$; and $x+\Delta x, y+\Delta y$ those of $t$. The area st generates by revolution an elementary ring, the volume of which is $\pi(y+\Delta y)^{2} \Delta x-\pi y^{2} \Delta x$; this may be put ultimately equal to $2 \pi y \Delta y \Delta x$. The centre of gravity of this ring is on the axis of $x$, and its abscissa is 
nleimntely $x$. Hence by proceeding as hefore we shall have ultimately

$$
\bar{x}=\frac{\int_{e}^{\Delta} \int_{0}^{\infty} y_{0} y x d x d y}{\int_{0}^{n} \int_{0 \rightarrow \infty}^{\infty} y d x d y},
$$

where $y=\psi(x)$ is the equation to the lower bounding curve and $y=\phi(x)$ to the upper, and $c$ and $h$ are the ahsciseas of the planes which bound the solid of revolution perpendicularly to its axis.

Similarly, if the solid is formed by revolving the area indin lad befween two curves round the axis of $y$, we shall have

$$
\bar{y}=\frac{\int_{d}^{x} \int_{\infty}^{\infty x} x y d y d x}{\int_{\infty}^{\infty} x d y d x} .
$$

Or we may uae polar formula. Suppose the figure in Art. 122 to revolve round the axis of $x$; let $r, \theta$ be the polar $c 0-$ ordinates of $s$; and $r+\Delta r, \theta+\Delta \theta$ those of $\ell$. The volume of the ring generated by the revolution of the area st is ultimately $2 \pi r \sin \theta r \Delta r \Delta \theta$; and the abscissa of the centre of gravity of the ring is ultimately $r \cos \theta$. Hence

$$
\bar{x}=\frac{\iint r^{2} \sin \theta \cos \theta d \theta d r}{\iint r^{3} \sin \theta d \theta d r} .
$$

Similarly, if the figure revolve round the axis of $y$

$$
\bar{y}=\frac{\pi r^{3} \cos \theta \sin \theta d \theta d r}{\iint r^{3} \cos \theta d \theta d r} \text {. }
$$

We have hitherto sasumed the solid of revolution to be of uniform density; if this be not the case the formula must the modified. For example, take the first formula in the present Article; suppose that $\rho$ denotes the density at the point $(x, y)$. Then the mass of the ring consilered will be ultimately $2 \pi \rho y \Delta y \Delta x$. Hence 


$$
\bar{x}=\frac{\int_{0}^{h} \int_{\psi(x)}^{\mu(x)} \rho y x d x d y}{\int_{0}^{h} \int_{\psi(x)}^{\beta(x)} \rho y d x d y} .
$$

And $\rho$ being supposed a known function of $x$ and $y$, the integrations present no theoretical difficulty.

Similarly the polar formula may be modified. For example, instead of the formula given above for $\bar{x}$ we now obtain

$$
\bar{x}=\frac{\iint \rho r^{2} \sin \theta \cos \theta d \theta d r}{\iint \rho r^{2} \sin \theta d \theta d r} .
$$

In this case $\rho$ must be expressible as a function of $r$ and $\theta$, in order that the integrations may be practicable. The most common cases are two; in one the density depends only on the distance from a fixed point in the axis of revolution, so that by taking this point as origin $\rho$ is a function of $r$; in the other case the density depends only on the distance from the axis of revolution, so that $\rho$ is a function of $r \sin \theta$.

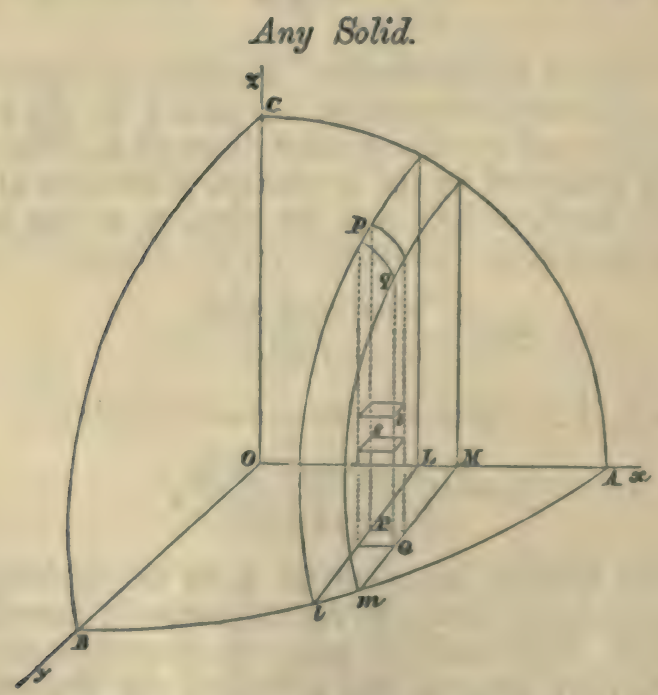

128. To find the centre of gravity of a solid we divide it into elements as follows: draw a series of planes perpen- 
dieular to the axis of $x$, then two consceutive planes will include between them a slice such as Lplmq MI in the figure; draw a second series of planes perpendicular to the axis of $y$. then each slice is divided into strips such as $B_{p q} Q$ in the figure; lastly, draw planes perpendicular to the axis of $\mathbf{z}$, then each strip is divided into parallelepipeds such as ot in the figure. Let $x, y, z$ be the co-ordinates of $s$ and $x+\Delta x$, $y+\Delta y, z+\Delta z$ those of $\ell$; then $\Delta x \Delta y \Delta z$ is the volume of $n$, and as the co-ordinates of its centre of gravity are ultimately $x, y$, and $z$, we have

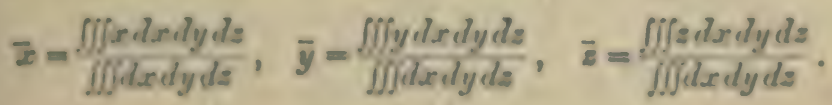

129. In applying the above formula to examples, great eare is neceseary in nesigning proper limits to the integrations; this we shall illustrate by Examples

Ex. 1. Find the centre of gravity of the eighth part of an ellipsoid cut off by three principal planes.

Let the equation to the surface be

$$
\frac{x^{2}}{a^{3}}+y^{2}+\frac{z^{2}}{y^{3}}=1 \text {. }
$$

Then the equation to the curve in which the surface meets the plane of $(x, y)$ is

$$
\frac{x^{2}}{a^{3}}+\frac{y^{3}}{b^{3}}=1
$$

Integrate first with respect to $z$, and take for the limits $z=0$ and $z=c \sqrt{ }\left(1-\frac{x^{2}}{a^{3}}-\frac{y^{2}}{b^{3}}\right)$; we thus include all the elements like st which form the strip $P$ pQ . Next integrate with respect to $y$, and take for the limits $y=0$ and $y=b \sqrt{ }\left(1-\frac{x^{2}}{a^{2}}\right)$; we thus include all the strips like $\Gamma_{p q} Q$ which form the slice Lplmi.M. Lastly integrate with reapect to $x$, and take for 
the limits $x=0$ and $x=a$; we thus include all the slices $L p \operatorname{lm} q M$ which form the solid we are considering. Hence

$$
\bar{x}=\frac{\int_{0}^{a} \int_{0}^{n_{1}} \int_{0}^{z_{1}} x d x d y d z}{\int_{0}^{a} \int_{0}^{y_{1}} \int_{0}^{z_{2}} x d x d y d z},
$$

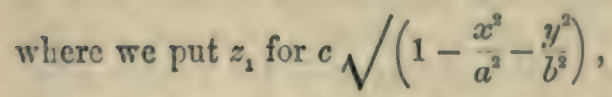

$$
\text { and } y_{1} \text { for } b \sqrt{\left(1-\frac{x^{2}}{a^{2}}\right)} \text {. }
$$

Now

$$
\int_{0}^{z_{1}} d z=z_{1}=c \sqrt{\left(1-\frac{x^{2}}{a^{2}}-\frac{y^{2}}{b^{2}}\right)}
$$

therefore $\bar{x}=\frac{\int_{0}^{a} \int_{0}^{y_{1}} x \sqrt{\left(1-\frac{x^{2}}{a^{2}}-\frac{y^{2}}{b^{2}}\right)} d x d y}{\int_{0}^{a} \int_{0}^{y_{1}} \sqrt{\left(1-\frac{x^{2}}{a^{2}}-\frac{y^{2}}{b^{2}}\right) d x d y}}$.

And $\int_{0}^{y_{1}} \sqrt{ }\left(1-\frac{x^{2}}{a^{2}}-\frac{y^{2}}{b^{2}}\right) d y, \quad$ or $\frac{1}{b} \int_{0}^{y_{1}} \sqrt{ }\left(y_{1}^{2}-y^{2}\right) d y=\frac{\pi y_{1}^{2}}{4 b}$;

therefore $\quad \bar{x}=\frac{\int_{0}^{a} x y_{1}{ }^{2} d x}{\int_{0}^{a} y_{2}{ }^{2} d x}=\frac{\int_{0}^{a}\left(1-\frac{x^{2}}{a^{8}}\right) x d x}{\int_{0}^{a}\left(1-\frac{x^{2}}{a^{8}}\right) d x}=\frac{3 a}{8}$.

Similarly $\quad \bar{y}=\frac{3 b}{8}, \quad \bar{z}=\frac{3 c}{8}$.

We may in this example effect the integrations with equal simplicity in any order we please; if we integrate first for $x$, then for $y$, and lastly for $z$, we shall have

$$
\bar{x}=\frac{\int_{0}^{c} \int_{0}^{y_{1}} \int_{0}^{x_{1}} x d z d y d x}{\int_{0}^{c} \int_{0}^{y_{1}} \int_{0}^{x_{1}} d z d y d x}
$$


where $x_{1}$ stands for $a \sqrt{ }\left(1-\frac{z^{2}}{c^{2}}-\frac{y^{2}}{b^{2}}\right)$,

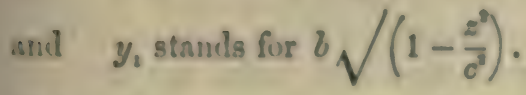

This will be easily seen by drawing a figure so as to make the planes bounding the slice parallel to that of $(x, y)$, and the edges of the strip parallel to the axis of $x$.

Ex. 2. Ixt it be required to find the centre of gravity of the solid bounded ly the planes $z=\beta x, z=\gamma x$, and the cylinler $y^{3}=2 a x-x^{2}$. We shall have

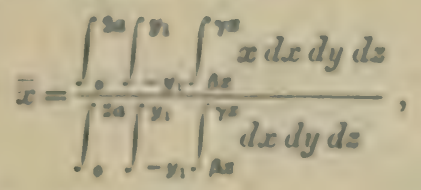

where $y_{2}$ is put for $\sqrt{ }\left(2 a x-x^{2}\right)$.

Now

therefore

$$
\begin{aligned}
& \int_{\beta=}^{\gamma_{z}} d z=(\gamma-\beta) x, \\
& \bar{x}=\frac{\int_{0}^{2 a} \int_{-y_{1}}^{y_{1}} x^{2} d x d y}{\int_{0}^{2 a} \int_{-y_{1}}^{y_{1}} x d x d y} .
\end{aligned}
$$

Also

$$
\begin{gathered}
\int_{-y_{1}}^{y_{1}} d y=2 \sqrt{ }\left(2 a x-x^{2}\right) ; \\
\bar{x}=\frac{\int_{0}^{2 a} x^{2} \sqrt{ }\left(2 a x-x^{2}\right) d x}{\int_{0}^{2 a} x \sqrt{\left(2 a x-x^{2}\right) d x}}=\frac{5 \pi}{4} .
\end{gathered}
$$

(See Integral Calculus, Ex. 5 to Chap. in.)

Similarly we may find

$$
\bar{y}=0, \quad \bar{z}=\frac{5 a(\beta+\gamma)}{8} .
$$


130. It is often convenient to divide a solid into polar elements.

Let a series of planes be drawn through the axis of $z$; the solid is thus divided into wedge-shaped slices such as COML. Let a series of right cones be described round the axis of $z$ having their vertices at $O$; thus each slice is divided into pyramidal solids like $O P Q S$. Lastly, let a series of concentric

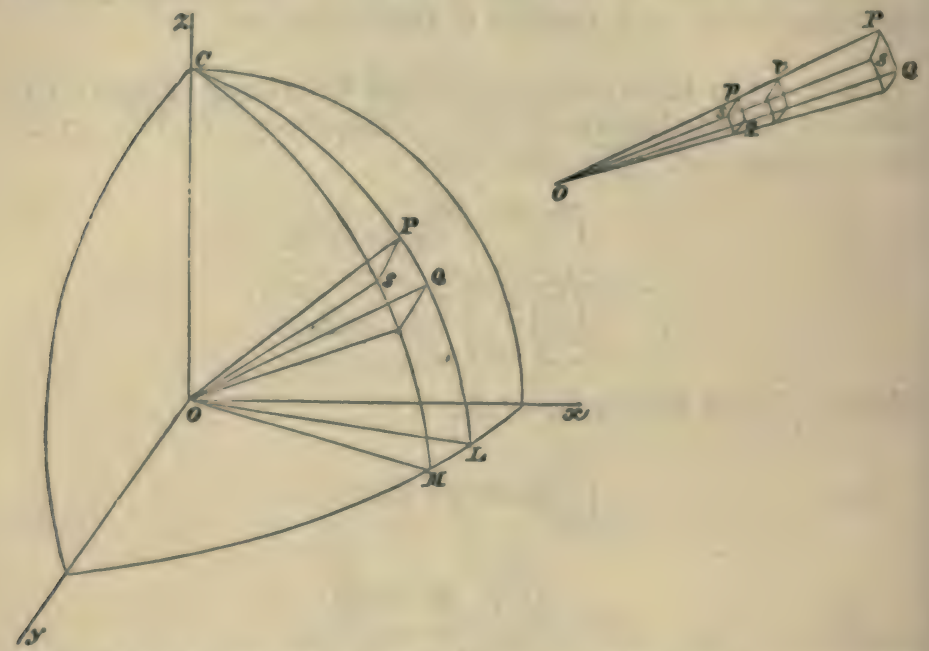

spheres be described round $O$ as centre; thus each pyramid is divided into elements similar to $p q s t$.
Let

$$
\begin{aligned}
& x O L=\phi, \\
& L O M=\Delta \phi,
\end{aligned}
$$$$
C O P=\theta \text {, }
$$$$
O p=r,
$$$$
P O Q=\Delta \theta,
$$$$
p t=\Delta r \text {. }
$$

Then $p q$ is the arc of a circle of which the radius is $r$ and the angle $\Delta \theta$; therefore $p q=r \Delta \theta$.

Also $p s$ is the arc of a circle of which the radius is $r \sin \theta$ and the angle $\Delta \phi$; therefore $p s=r \sin \theta \Delta \phi$.

Hence, since the element $p q s t$ is ultimately a parallelepiped, its volume is $r^{2} \sin \theta \Delta \theta \Delta \phi \Delta r$.

Also the co-ordinates of its centre of gravity are ultimately $r \cos \phi \sin \theta, r \sin \phi \sin \theta$, and $r \cos \theta$. Hence supposing its density to be $\rho$, we have 


$$
\begin{aligned}
& \bar{x}=\frac{\int I i \rho r^{2} \sin ^{2} \theta \cos \phi d \phi d \theta d r}{\int ! \int \rho r^{2} \sin \theta d \phi d \theta d r}, \\
& \bar{y}=\frac{\int i I \rho r^{2} \sin ^{2} \theta \sin \phi d \phi d \theta d r}{\int I \int \rho r^{3} \sin \theta d \phi d \theta d r}, \\
& \bar{z}=\frac{\int i \int \rho r^{2} \sin \theta \cos \theta d \phi d \theta d r}{\iiint \rho r^{2} \sin \theta d \phi d \theta d r} .
\end{aligned}
$$

131. Kx. 1. Apply the preceling formula to find the centre of gravity of a hemisphere whose density varies as the $n^{\mathrm{ta}}$ power of the distance from the eentre.

Take the axis of a perpendicular to the plane base of the hemisphere. Let a be the radius of the hemisphere, and $\rho=\mu r^{\circ}$, where $\mu$ is a constant. First integrate with respect to r frum o to $a$; we thus inclute all the elements like pqut comprised in the pyrnmid OPQS. Next integrate with respect to a from 0 to $3 \pi$, we thus include all the pyramids in the slice COMT. Finally, integrate from $\phi=0$ to $\phi=2 \pi$; we thus include all the slices. Thus

\section{$\bar{x}$ and $\bar{y}$ each $=0$.}

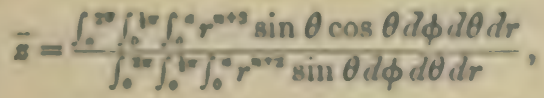

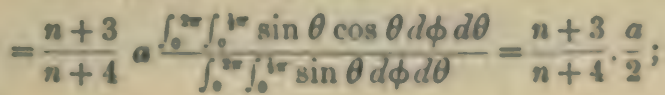

E.x. 2. A right cone has its rertex on the surface of a sphere and its axis coincident with a diameter of the sphere, find the centre of gravity of the solid incladed between the cone and sphere. Take the axis of $z$ coincident with that of the cone; suppose $a$ the radius of the sphere, $\beta$ the semivertical angle of the cone. The polar equation to the sphere is $r=2 a \cos \theta$, and to the cone $\theta=\beta$. Hence we have

$$
\bar{\Sigma}=\frac{\int_{0}^{2 \pi} \int_{0}^{\beta} \int_{0}^{20 \cos \theta} r^{2} \cos \theta \sin \theta d \phi d \theta d r}{\int_{0}^{2 \pi} \int_{0}^{\beta} \int_{0}^{20 \cos \theta} r^{2} \sin \theta d \phi d \theta d r} ;
$$

$\bar{x}$ and $\bar{y}$ each $=0$. 


\section{Curve.}

132. Suppose a circle of variable radius to move so that its centre describes a given curve and its plane is always perpendicular to the tangent line of the curve, we may require the centre of gravity of the solid generated. The simplest case is that in which the radius is constant and the solid of uniform density; the result depends solely on the nature of the curve described by the centre of the circle, and for shortness the process is called finding the centre of gravity of a curve.

Let $B P Q E$ be a plane curve; $B P$ the length measured from some fixed point $B$, $B P=s, P Q=\Delta s ; x, y$ the co-ordinates of $P$. Let $k$ denote the area of a transverse section; then the volume of the element $P Q$ is $k \Delta s$, and the co-ordinates of its centre of gravity are ultimately $x$ and $y$. Hence

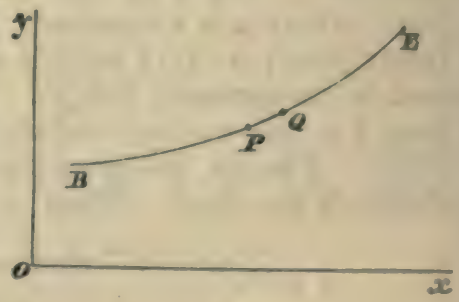

$$
\begin{aligned}
& \bar{x}=\frac{\int k x d s}{\int k d s}=\frac{\int x d s}{\int d s} \ldots \text { (1) if } k \text { be constant, } \\
& \bar{y}=\frac{\int k y d s}{\int k d s}=\frac{\int y d s}{\int d s} \ldots \text { (2). }
\end{aligned}
$$

Since $\frac{d s}{d x}=\sqrt{ }\left\{1+\left(\frac{d y}{d x}\right)^{2}\right\}$, we may also write

$$
\bar{x}=\frac{\int x \sqrt{\left\{1+\left(\frac{d y}{d x}\right)^{2}\right\} d x}}{\int \sqrt{\left\{1+\left(\frac{d y}{d x}\right)^{2}\right\} d x}}, \bar{y}=\frac{\int y \sqrt{\left\{1+\left(\frac{d y}{d x}\right)^{2}\right\}} d x}{\int \sqrt{\left\{1+\left(\frac{d y}{d x}\right)^{2}\right\} d x}} \ldots
$$

From the equation to the curve $y$ and $\frac{d y}{d x}$ are known in 
terms of $x$; their values must be substituted in the preceding expressions and the integrations then effected.

If we use polar co-ordinates we have $x=r \cos \theta, y=r \sin \theta$, and $\frac{d s}{d \theta}=\sqrt{ }\left\{p^{3}+\left(\frac{d r}{d \theta}\right)^{2}\right\}$.

Hence

$x=\frac{\int r \cos \theta \sqrt{\left\{r^{2}+\left(\frac{d r}{d \theta}\right)^{2}\right\}} d \theta}{\int \sqrt{\left\{r^{2}+\left(\frac{d r}{d \theta}\right)^{2}\right\} d \theta}}, \bar{y}=\frac{\int r \sin \theta /\left\{r^{2}+\left(\frac{d r}{d \theta}\right)^{2}\right\} d \theta}{\int \sqrt{\left\{r^{2}+\left(\frac{d r}{d \theta}\right)^{2}\right\} d \theta}}$

for $r$ snd $\frac{d r}{d t}$ we must substitute their values in terms of $\theta$ given by the equation to the curve.

133. Fx. 1. A straight rod of uniform thickness and density.

Taking the origin on the line we have $y=\beta x$, where $\beta$ is constant; hence, ly equations (3) of Art. 132, supposing the origin to be at one end of the rod and $h$ the abscissa of the other end,

$$
\bar{x}=\frac{\int_{0}^{2} x d x}{\int_{0}^{2} d x}=\frac{h}{2}, \quad \bar{y}=\frac{\beta \int_{0}^{2} x d x}{\int_{0}^{2} d x}=\frac{\beta h}{2} .
$$

That is, the centre of gravity is the middle point of the rod.

Ex. 2. Supposo the transverse section of the rod to vary as the $n^{\text {th }}$ power of the distance from one end. Take the origin at this end, and suppose the axis of $x$ to coincile with the axis of the rod; then $\vec{y}=0$, and in equation (1) of Art. 132 we put $\mu x^{*}$ for $k$, where $\mu$ is constant. Hence, if $h$ be the length of the rod,

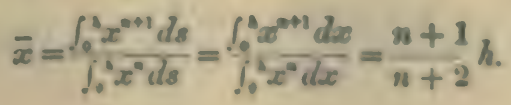


Ex. 8. An arc of a circle.

Take the origin at the centre of the circle, and the axis of $x$ bisecting the arc. Then $\bar{y}=0$; and supposing $2 x$ to be the angle subtended at $O$ by the given are, and $a$ the radius of the circle, we have, by Art. 132, equation (4),

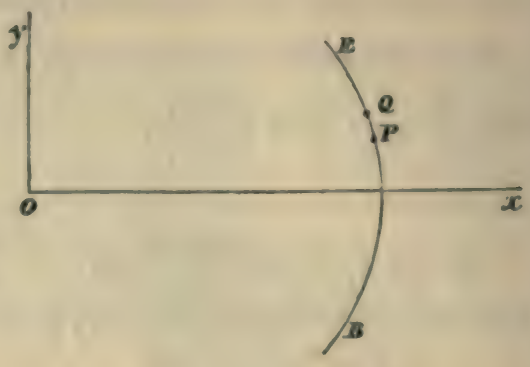

$$
\bar{x}=\frac{a^{2} \int_{-\alpha}^{\alpha} \cos \theta d \theta}{a \int_{-\alpha}^{a} d \theta}=\frac{a \sin \alpha}{\alpha} .
$$

Ex. 4. The arc of a semicycloid.

Take the origin at the vertex, and the axis of $y$ a tangent there; then $\left(\frac{d y}{d x}\right)^{2}=\frac{2 a-x}{x}$ : hence

$$
\begin{aligned}
& x=\frac{\int_{0}^{2 a} x \sqrt{\left(\frac{2 a}{x}\right) d x}}{\int_{0}^{2 a} \sqrt{\left(\frac{2 a}{x}\right) d x}}=\frac{\int_{0}^{2 a} x^{\frac{1}{4}} d x}{\int_{0}^{2 a} x^{-1} d x}=\frac{\frac{2}{3}(2 a)^{2}}{2(2 a)^{\frac{1}{2}}}=\frac{2 a}{3}, \\
& \bar{y}=\frac{\int_{0}^{2 a} y \sqrt{\left(\frac{2 a}{x}\right) d x}}{\int_{0}^{2 a} \sqrt{\left(\frac{2 a}{x}\right) d x}}=\frac{\int_{0}^{2 a} \frac{y}{\sqrt{x}} d x}{\int_{0}^{2 a} \frac{d x}{\sqrt{x}}} .
\end{aligned}
$$

Now $\int \frac{y}{\sqrt{x}} d x=2 \int y \frac{d \sqrt{ } x}{d x} d x=2 y \sqrt{x}-2 \int \sqrt{ } x \frac{d y}{d x} d x$

$$
=2 y \sqrt{ } x-2 \int \sqrt{ }(2 a-x) d x=2 y \sqrt{ } x+\frac{f}{3}(2 a-x)^{1} ;
$$

therefore

$$
\int_{0}^{23} \frac{y}{\sqrt{ } x} d x=2 \pi a(2 a)^{\frac{1}{2}}-\frac{4}{3}(2 a)^{1} ;
$$

therefore

$$
\bar{y}=\frac{2 \pi a(2 a)^{\frac{1}{3}}-\frac{4}{3}(2 a)^{1}}{2(2 a)^{3}}=\left(\pi-\frac{1}{3}\right) a \text {. }
$$


Ex. 5. The curve $y=10\left(\sigma^{\frac{2}{2}}+e^{-2}\right)$.

If $s$ denote the length of an are of the curve measured from the point whose co-ardinates are $0, c$, to the point $\left(x^{\prime}, y\right)$, we have for the co-urdinates of its centre of gravity

$$
\bar{x}=\frac{\int_{0}^{x} x \frac{d x}{d x} d x}{y^{2}}, \quad \bar{y}=\frac{\int_{0}^{e} y \frac{d x}{d x} d x}{3} .
$$

Now

$$
\frac{d y}{d x}=\frac{1}{2}\left(e^{i}-e^{-6}\right) \text {, }
$$

Qlicrefore

$$
1+\left(\frac{d y}{d x}\right)^{3}=\frac{1}{4}\left(e^{\frac{d}{2}}+e^{-\frac{1}{2}}\right)^{2},
$$

and

$$
\begin{aligned}
& \frac{d x}{d x}=\sqrt{ }\left\{1+\left(\frac{d y}{d x}\right)\right\}=\frac{1}{2}\left(e^{d}+e^{-\frac{b}{b}}\right) \\
& s=\frac{c}{2}\left(e^{d}-e^{-i}\right), \text { and } s^{\prime}=\frac{c}{2}\left(e^{e}-e^{-b}\right) .
\end{aligned}
$$

Ilso

$$
\begin{aligned}
& \int x \frac{d s}{d x} d x=\frac{1}{2} \int x\left(e^{\frac{1}{3}}+e^{-\frac{1}{6}}\right) d x \\
& =\frac{c x}{2}\left(e^{\vdots}-e^{-\frac{a}{c}}\right)-\frac{c}{2} \int\left(e^{\frac{a}{2}}-e^{-i}\right) d x \\
& =\frac{c x}{2}\left(e^{\frac{\pi}{b}}-e^{-\frac{\pi}{6}}\right)-\frac{c^{2}}{2}\left(e^{2}+e^{-i}\right) \text {; }
\end{aligned}
$$

therefore $\int_{0}^{e} x \frac{d s}{d x} d x=\frac{c x^{2}}{2}\left(e^{\frac{2}{6}}-e^{-\frac{x}{5}}\right)-\frac{c^{2}}{2}\left(e^{2}+e^{-e^{2}}\right)+c^{2}$

and

$$
\begin{gathered}
=x^{\prime} s^{\prime}-c y^{\prime}+c^{\prime}, \\
\bar{x}=x^{\prime}-\frac{c\left(y^{\prime}-c\right)}{s^{\prime}} .
\end{gathered}
$$

Also

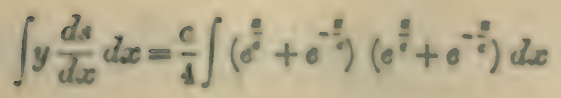


therefore

$$
=\frac{c}{4} \int\left(e^{\frac{2 x}{c}}+2+e^{-\frac{2 x}{0}}\right) d x=\frac{c^{2}}{8}\left(e^{\frac{2 x}{c}}-e^{-\frac{2 x}{c}}\right)+\frac{c x}{2} ;
$$

$$
\begin{aligned}
\int_{0}^{x^{\prime}} y \frac{d s}{d x} d x & =\frac{e^{\prime}}{8}\left(e^{\frac{y^{\prime}}{e}}-e^{-\frac{2 x}{2}}\right)+\frac{c x^{\prime}}{2} \\
& =\frac{y^{\prime} s^{\prime}}{2}+\frac{c x^{\prime}}{2},
\end{aligned}
$$

and

$$
\bar{y}=\frac{y^{\prime}}{2}+\frac{c x^{\prime}}{2 s^{\prime}} \text {. }
$$

134. If the curve be of double curvature, the formula (1) and (2) of Art. 132 still hold; in order to effect the integrations we may use the formula

$$
\frac{d s}{d z}=\sqrt{ }\left\{1+\left(\frac{d x}{d z}\right)^{2}+\left(\frac{d y}{d z}\right)^{2}\right\}
$$

and from the two equations to the curve we must find $\frac{d x}{d z}$ and $\frac{d y}{d z}$ in terms of $z$. (See Integral Calculus, Art. 120.) For example, in the helix

$$
x=a \cos n z, \quad y=a \sin n z ;
$$

therefore

$$
\begin{gathered}
\frac{d s}{d z}=\sqrt{ }\left(1+n^{2} a^{2}\right), \\
\bar{x}=\frac{\int \sqrt{ }\left(1+n^{2} a^{2}\right) x d z}{\int \sqrt{ }\left(1+n^{2} a^{3}\right) d z}=\frac{\int a \cos n z d z}{\int d z} .
\end{gathered}
$$

If we take for the limits $z=0$ and $z=h$, we have

Similarly

$$
\begin{aligned}
& \bar{x}=\frac{a \sin n h}{n h} . \\
& \bar{y}=\frac{a(1-\cos n h)}{n h}, \quad \bar{z}=\frac{1}{2} h .
\end{aligned}
$$




\section{Sarfuce of Recolusion.}

13.5. Iat $B P Q E$ be a curve which by revolving round the axis of $x$ generates a surface Suppose a Bliell of which this surnace in the exterior boundary, and of which the interior boundary is ancther surface of revoluiin rouml the axis of $x$ in1. finitely near to the furmer. dieguired the centre of gravity of a portion of this shell cut of by flanes perpendicular to

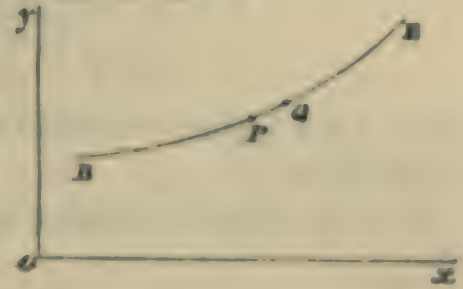
the axis of $x$.

Let $P_{,} Q$, be adjacent poiss in the exterior generating earve; stipose $B$ a fised point in the curve, let $T P P=s$, and $P Q=\Delta z$; let $x, y$ be the co-ordinates of $P ; k$ the thickness of the shell at $R$. The volume of the element contained between two plases perpendieular to the axis of $x$ through $P$ and $Q$ respectirely is nlfimately $2 \pi y k \mathrm{ds}$, and the abscissa of the eentre of gravity of this element is ultimately $x$; hence

$$
\bar{x}=\frac{\int 2 \pi y k \cdot x d s}{\int 2 \pi y k d s}=\frac{\int y x d s}{\mid y d s},
$$

if $k$ le constant.

$$
\begin{aligned}
& \text { Since } \frac{d x}{d x}=\sqrt{ }\left\{1+\left(\frac{d y}{d x}\right)^{2}\right\} \text {, we have } \\
& \qquad \bar{x}=\frac{\int_{0}^{4} y x \sqrt{\left\{1+\left(\frac{d y}{d x}\right)^{\prime}\right\} d x}}{\int_{0}^{x} y \sqrt{\left\{1+\left(\frac{d y}{d x}\right)^{2}\right\}}, d x},
\end{aligned}
$$

where end $h$ are the distances from the origin of the bounding planes.

Since the centre of gravity required is on the axis of $x$, wo need only the value of $x$ in urder to determine its position. ร. 8. 
Similarly, if the curve $B P Q E$ generates a surface by revolving round the axis of $y$, we have

$$
\bar{y}=\frac{\int_{c}^{n} x y \sqrt{\left\{1+\left(\frac{d y}{d x}\right)^{2}\right\}} d x}{\int_{c}^{n} x \sqrt{\left\{1+\left(\frac{d y}{d x}\right)^{2}\right\}} d x},
$$

where $c$ and $h$ denote as before the abscissæ of the extremities of the curve.

If we use polar co-ordinates, we have $x=r \cos \theta, y=r \sin \theta$, and

$$
\frac{d s}{d \theta}=\sqrt{\left\{r^{2}+\left(\frac{d r}{d \theta}\right)^{2}\right\}} ;
$$

thus if the curve revolves round the axis of $x$, we have

$$
\bar{x}=\frac{\int r^{2} \sin \theta \cos \theta \sqrt{\left\{r^{2}+\left(\frac{d r}{d \theta}\right)^{2}\right\} d \theta}}{\int r \sin \theta \sqrt{\left\{r^{2}+\left(\frac{d r}{d \theta}\right)^{2}\right\}} d \theta}
$$

and if the curve revolves round the axis of $y$, we have

$$
\bar{y}=\frac{\int r^{2} \cos \theta \sin \theta \sqrt{\left\{r^{2}+\left(\frac{d r}{d \theta}\right)^{2}\right\} d \theta}}{\int r \cos \theta \sqrt{\left\{r^{2}+\left(\frac{d r}{d \theta}\right)^{2}\right\} d \theta}} .
$$

The limits of the integrations are the values of $\theta$ which correspond to the extremities of the curve.

Ex. 1. A cylindrical surface.

Take the axis of the cylinder as the axis of $x$; then $y=$ the radius of the cylinder, and is constant; hence

$$
\bar{x}=\frac{\int_{c}^{h} x d x}{\int_{0}^{h} d x}=\frac{\frac{1}{2}\left(h^{2}-c^{2}\right)}{h-c}=\frac{h+c}{2} .
$$

Ex. 2. A spherical surface. 
BUEFACR.

Ilere

$$
\begin{aligned}
& y=\sqrt{\left(a^{2}-x^{2}\right),} \\
& \frac{d y}{d x}=-\frac{x}{\sqrt{\left(a^{3}-x^{2}\right)}} \text {, } \\
& \frac{d x}{d x}=\frac{a}{\sqrt{\left(a^{3}-x^{5}\right)}}=\frac{a}{y} \text {; } \\
& \bar{x}=\frac{\int \operatorname{cox} d x}{\operatorname{Cad} x}=\frac{0+A}{2} \text {. }
\end{aligned}
$$

Hence in bath these examples the centre of gravity is equidiveant from the two bounding planes.

Ex, 3. The surface of a cone.

Herw $y=x \tan \alpha$, where $\alpha$ is the semivertical angle,

$$
\begin{gathered}
\frac{d x}{d x}=\sec a, \\
x=\frac{C x \tan a x \sec \alpha d x}{\int_{c} x \tan \alpha \sec 2 d x}=\frac{2\left(h^{3}-c^{2}\right)}{3\left(h^{2}-c^{3}\right)}=\frac{2\left(h^{2}+h c+c^{2}\right) .}{3(h+c)} .
\end{gathered}
$$

Ex. 1. Suppose the cycloid

$$
y=\sqrt{ }\left(2 a x-x^{2}\right)+a \operatorname{vers}^{-1} \frac{x}{a}
$$

to revolve round the axis of $x$.

Here

thins

$$
\begin{aligned}
& \frac{d y}{d x}=\sqrt{\left(\frac{2 a-x}{x}\right), \quad \frac{d y}{d x}=\sqrt{\left(\frac{2 a}{x}\right)} ;} \\
& \bar{x}=\frac{\int_{-\infty}^{\infty} y x \sqrt{\left(\frac{2 a}{x}\right) d x}}{\int_{0}^{\infty} y \sqrt{\left(\frac{2 a}{x}\right) d x}}=\frac{\int_{0}^{\infty} y x^{6} d x}{\int_{0}^{\infty} y x^{-1} d x}
\end{aligned}
$$

Sow $\int y x^{d} d x=\frac{-y x^{4}}{3}-3 \int x^{1} \frac{d y}{d x} d x$

$$
=\frac{2 y \cdot x^{1}}{3}-\iint x \sqrt{ }(2 a-x) d x ;
$$


therefore $\quad \int_{0}^{2 a} y x^{\frac{1}{2}} d x=\frac{2 \pi a(2 a)^{1}}{3}-\frac{2}{3} \int_{0}^{2 a} x \sqrt{ }(2 a-x) d x$; and $\int x \sqrt{ }(2 a-x) d x=-\frac{2 x(2 a-x)}{3}+\frac{2}{3} \int(2 a-x)^{3} d x$

$$
=-\frac{2 x(2 a-x)^{\prime}}{3}-\frac{4}{15}(2 a-x)^{!}
$$

therefore

$$
\begin{aligned}
& \int_{0}^{a} x \sqrt{(2 a-x) d x=\frac{4}{15}(2 a)^{\frac{1}{3}}} \\
& \int_{0}^{\infty} y x^{\frac{1}{3}} d x=\frac{2 \pi a(2 a)^{3}}{3}-\frac{8}{45}(2 a)^{\frac{3}{3}}
\end{aligned}
$$

thus

Also

$$
\left.\int_{0}^{2 a} y x^{-\frac{1}{2}} d x=2 \pi a(2 a)^{\frac{1}{2}}-\frac{4}{3}(2 a)^{\frac{1}{3}} \text {, (see page } 142\right) \text {, }
$$

$$
\begin{aligned}
\bar{x} & =\frac{\frac{2 \pi a(2 a)^{1}}{3}-\frac{8}{45}(2 a)^{8}}{2 \pi a(2 a)^{\frac{1}{3}}-\frac{4}{3}(2 a)^{\frac{1}{3}}} \\
& =a \frac{\frac{4 \pi}{3}-\frac{32}{45}}{2 \pi-\frac{8}{3}}=\frac{\frac{2 a}{3}\left(\pi-\frac{8}{15}\right)}{\pi-\frac{4}{3}} .
\end{aligned}
$$

Ex. 5. Suppose the cycloid

$$
y=\sqrt{ }\left(2 a x-x^{2}\right)+a \operatorname{vers}^{-1} \frac{x}{a}
$$

to revolve round the axis of $y$, and that we require the centre of gravity of the surface generated by that half of the curve for which $y$ is positive.

Here

$$
\bar{y}=\frac{\int_{0}^{2 a} y x \sqrt{\left(\frac{2 a}{x}\right) d x}}{\int_{0}^{2 a} x \sqrt{\left(\frac{2 a}{x}\right) d x}}=\frac{\int_{0}^{2 a} y x^{\frac{1}{2}} d x}{\int_{0}^{2 a} x^{\frac{1}{2}} d x} .
$$

The value of the numerator was found in the preceding example; and

$$
\int_{0}^{9 \pi} x^{d} d x=\frac{2}{3}(2 a)
$$


BLIRPACR.

thereforo

$$
\begin{aligned}
\bar{y} & =\frac{\frac{2 \pi a}{3}(2 u)^{1}-\frac{8}{45}(2 a)^{3}}{\frac{2}{3}(2 a)^{1}} \\
& =a\left(\pi-\frac{8}{15}\right) .
\end{aligned}
$$

Ex. 6. Find the centre of gravity of the surface formed by revulving the curve $r=a(t+\cos \theta)$ round the initial lise. Ilere

$$
\frac{d r}{d \theta}=-a \sin \theta, \quad r^{2}+\left(\frac{d r}{d \theta}\right)^{2}=2 a^{2}(1+\cos \theta) \text {. }
$$

therefure

$$
\frac{d s}{d \theta}=\sqrt{ }\left\{r^{2}+\left(\frac{d r}{d \theta}\right)^{2}\right\}=2 a \cos \frac{\theta}{2} \text {. }
$$

Thus

$$
\bar{x}=\frac{\int_{0}^{\infty} r \sin \theta \cos \theta 2 a \cos \frac{\theta}{2} d \theta}{\int_{s}^{\infty} r \sin \theta 2 a \cos \frac{\theta}{2} d \theta}
$$

$$
=\frac{2 a \int_{0}^{\infty} \cos ^{8} \frac{\theta}{2}\left(2 \cos ^{2} \frac{\theta}{2}-1\right) \sin \frac{\theta}{2} d \theta}{\int_{0}^{2} \cos ^{\circ} \frac{\theta}{2} \sin \frac{\theta}{2} d \theta} .
$$

Nuw $\int \cos ^{\circ} \frac{\theta}{2}\left(2 \cos ^{2} \frac{\theta}{2}-1\right) \sin \frac{\theta}{2} d \theta=-\frac{4}{9} \cos ^{\circ} \frac{\theta}{2}+\frac{2}{8} \cos ^{\circ} \frac{\theta}{2}$;

therefure $\int_{0}^{\infty} \cos ^{6} \frac{\theta}{2}\left(2 \cos ^{8} \frac{\theta}{2}-1\right) \sin _{2}^{\theta} d \theta=\frac{4}{9}-\frac{2}{i}$.

Sinilarly

$$
\begin{aligned}
& \int_{0} \cos ^{\circ} \frac{\theta}{2} \sin \frac{\theta}{2} d \theta=\frac{2}{5}, \\
& \bar{x}=\frac{2 a\left(\frac{4}{9}-\frac{2}{7}\right)}{\frac{2}{5}}=\frac{50 a}{63} .
\end{aligned}
$$

\section{Any Swerface.}

136. Iet there be a shell having any given surface for one of its boundaries, and suppose its thickness indelinitely 
small. Let $x, y, z$ be the co-ordinates of any point of the given surface, $k$ the thickness at that point, $\Delta S$ the area of an element of the surface there, then $k \Delta S$ is ultimately the volume of this element, and $x, y, z$ the co-ordinates of its centre of gravity; hence

$$
\bar{x}=\frac{\int k x d . S}{\int k d S},
$$

and similar expressions hold for $\bar{y}$ and $\bar{z}$.

It may be shewn (see Integral Calculus, Art. 170) that if we take $\Delta S$ such that its projection on the plane of $(x, y)$ is the rectangle $\Delta x \Delta y$,

$$
\begin{aligned}
& \frac{\Delta S}{\Delta x \Delta y}=\sqrt{ }\left\{1+\left(\frac{d z}{d x}\right)^{2}+\left(\frac{d z}{d y}\right)^{2}\right\} \text { ultimately. } \\
& \text { Hence } \quad \bar{x}=\frac{\iint k x \sqrt{ }\left\{1+\left(\frac{d z}{d x}\right)^{2}+\left(\frac{d z}{d y}\right)^{2}\right\} d x d y}{\iint k \sqrt{\left\{1+\left(\frac{d z}{d x}\right)^{2}+\left(\frac{d z}{d y}\right)^{2}\right\} d x d y}}
\end{aligned}
$$

Ex. The surface of the eighth part of a sphere.

Here

Hence

$$
x^{2}+y^{2}+z^{2}=a^{2},
$$

$$
\begin{gathered}
\sqrt{\left\{1+\left(\frac{d z}{d x}\right)^{2}+\left(\frac{d z}{d y}\right)^{2}\right\}=\frac{a}{\sqrt{\left(a^{2}-x^{2}-y^{2}\right)}}} \cdot \\
\bar{x}=\frac{\iint \frac{x d x d y}{\sqrt{\left(a^{2}-x^{2}-y^{2}\right)}}}{\iint \frac{d x d y}{\sqrt{\left(a^{2}-x^{2}-y^{2}\right)}}}
\end{gathered}
$$

First integrate with respect to $y$ from $y=0$ to $y=\sqrt{ }\left(a^{2}-x^{2}\right)$; we thus include all the elements that form the strip of surface of which $L / m M$ is the projection on the plane of $(x, y)$; see fig. to Art. 128.

Now

$$
\begin{gathered}
\int_{0}^{V\left(a^{2}-x\right)} \frac{d y}{\sqrt{\left(a^{2}-x^{2}-y^{2}\right)}}=\frac{1}{2} \pi ; \\
\bar{x}=\frac{\int \frac{1}{2} \pi x d x}{\int \frac{1}{2} \pi d x}=\frac{\int x d x}{\int d x} .
\end{gathered}
$$

therefore

The limits of the integration for $x$ are 0 and $a$;

therefore

Similarly

$$
\begin{gathered}
\bar{x}=\frac{1}{2} a . \\
\bar{y}=\frac{1}{2} a, \quad \bar{z}=\frac{1}{2} a .
\end{gathered}
$$


13i. In the preceling Arteles we have given the usual formula for finding the centres of gravieg of bedies, but partiralar eases may coceur which may be most eonvenientig trated by sprecial metheds. We add some examples.

(1) A circle revolves round a tangent line through an

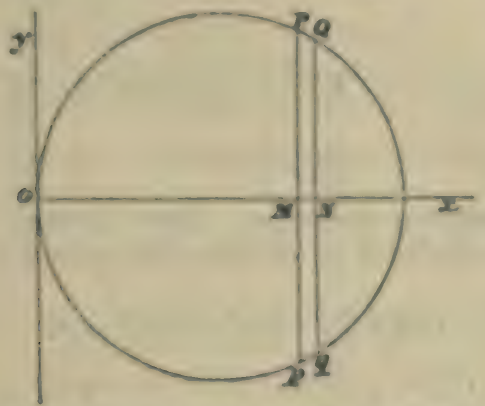

angle of $150^{\circ}$, find the centre of gravity of the solid generated. Iat $Q y$ be the tangent line about which the circle revolves, and let the plane of the paper bisect the solid; the centre of eravity will therefore lie in the axis of $x$. Ist $O M=x$, $M T^{\prime}=y=\sqrt{ }\left(2 a x-x^{3}\right), M N=\Delta x$. The figure $P P_{T l}$ will hy its revolution generate a semi-cylindrieal shell, whose volume is ultimately $2 y \pi x \perp x$; the centre of gravity of this shell will be in the axis of $x$ at a distance $\frac{2 x}{\pi}$ from $O$ (see Art. 133, li.x. 3) ;

therefore

$$
\begin{aligned}
x & =\frac{\int_{0}^{2 n} \frac{2 x}{\pi} 2 y \pi x d x}{\int_{0}^{\infty} 2 y \pi x d x}=\frac{\frac{2}{\pi} \int_{0}^{\infty} y x^{3} d x}{\int_{0}^{\infty} y x d x} \\
& =\frac{2}{\pi} \int_{0}^{\infty} x^{2} \sqrt{\left(2 a x-x^{2}\right) d x} x \sqrt{\left(2 a x-x^{2}\right) d x}
\end{aligned}
$$

It will be found that $\bar{x}=\frac{3 a}{2 \pi}$. 
(2) The density of a right cone varies as the $n^{\text {th }}$ power of the distance from the axis; find the centre of gravity of the cone.

Let $O A B$ be the right-angled triangle which by revolving

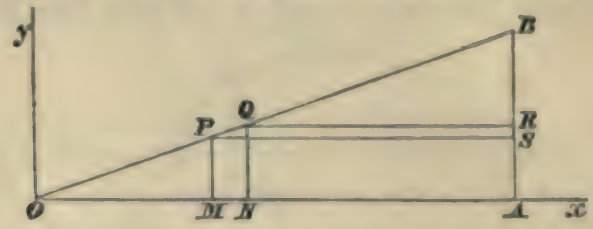

round $O x$ generates the cone. Let $P S$ and $Q R$ be drawn parallel to the axis of $x$ at distances $y$ and $y+\Delta y$ respectively. Let

$$
O A=h, \text { angle } B O A=\alpha .
$$

Then $\quad O M=y \cot \alpha, \quad P S=h-y \cot \alpha$.

The volume of the cylindrical shell generated by the revolution of $P Q R S$ round $O x$ is ultimately

$$
2 \pi y \Delta y(h-y \cot \alpha) \text {. }
$$

Its density is $\mu y^{n}$, where $\mu$ is constant; therefore, its mass is

$$
2 \pi \mu y^{n+1} \Delta y(h-y \cot \alpha) \text {. }
$$

The distance of its centre of gravity from $O$ is ultimately (sec Art. 135, Ex. 1)

$$
\frac{1}{2}(O M+O A), \text { that is } \frac{1}{2}(h+y \cot \alpha) ;
$$

therefore $\bar{x}=\frac{\int_{0}^{h \tan \alpha} 2 \pi \mu y^{n+1}(h-y \cot \alpha) \frac{1}{2}(h+y \cot \alpha) d y}{\int_{0}^{h \tan \alpha} 2 \pi \mu y^{n+1}(h-y \cot \alpha) d y}$

$$
=\frac{\frac{1}{2} \int_{0}^{h \tan \alpha} y^{n+1}\left(h^{2}-y^{2} \cot ^{2} \alpha\right) d y}{\int_{0}^{h \tan \alpha} y^{n+1}(h-y \cot \alpha) d y} ;
$$

and the integrations can be easily performed. 
(3) A shell has for its outer and inner boundaries two similar and similarly situnted ellipooids; required the centre of grarity of the eighth part of it includen between three prineipal planes. Let $a, 8, c$ be the semi-axes of the exterior ellipmid, ra, $r b$, re those of the inner ellipsouid, $r$ being a quantity less than unity.

If $a, b, c$ be the semi-axes of an ellipsoid; the volume of the eighth part is trabice, and the co-ordinates of its centre of

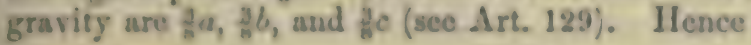

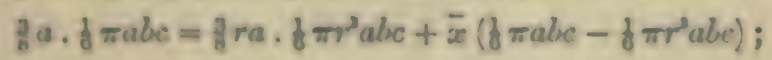

therefore

$$
\bar{x}=\frac{3 a\left(1-r^{2}\right)}{1-r^{3}}=\frac{1}{b} a \cdot \frac{1+r+r^{3}+r^{3}}{1+r+r^{2}} .
$$

If re suppose the shell indefinitely thin, we must put $r=1$, and then $x=\frac{1}{2} a$. Similar resules may be found for $y$ and $z$.

(1). An ellipeoia is composed of an infinite number of indeftritely thin shells; each shell has for its outer and inner bounlaries two similar and similarly situated ellipsoids: the dewity of each shell is constant, bint the density varies from shell to shell according to a given law; determine the centre of gravity of the eighth part of the ellipsoid included between three principal planes.

Iet $x_{1} y_{1}=$ represent the three semi-axes of an ellipsoid; then the volume of the ellipaoid is $\frac{4 \pi}{3}$ xyz. Supposo that $y=m x$ and $z=n x$, where $m$ and $n$ are constants, then the volume becomes $\frac{4 \pi m n}{3} x^{3}$, and if there be a similar ellipsoid having $x+\Delta x$ for the semi-axis cornesponding to the semiaxis $x$ of the first cllipsoid, the volume of the second ellipsoid will be $\frac{4 \pi m n}{3}(x+\Delta x)^{2}$. Hence the volume of a shell bounded by two similar and similarly situated ellipsoids may bo deroted by $\frac{4 \pi m n}{3}\left((x+\Delta x)^{3}-x^{2}\right)$, and therefore by $4 \pi m n x^{2} 1 x$ when the thickness is indefinitely diminished. Let $\phi(x)$ denote the density of the shell, then its mass is $\left.4 \pi m n \phi(x) x^{\prime}\right\rfloor x$. 
Thus the mass of the eighth part of the shell is $\frac{\pi m n}{2} \phi(x) x^{2} \Delta x$. And the abscissa of the centre of gravity of the shell measured along the semi-axis $x$ is $\frac{x}{2}$, by the preceding example. Thus for the abscissa $\bar{x}$ of the centre of gravity we have

$$
\begin{aligned}
\bar{x} & =\frac{\int_{0}^{a} \frac{\pi m n}{2} \phi(x) \frac{x}{2} x^{2} d x}{\int_{0}^{a} \frac{\pi m n}{2} \phi(x) x^{2} d x} \\
& =\frac{\frac{1}{2} \int_{0}^{a} x^{3} \phi(x) d x}{\int_{0}^{a} x^{2} \phi(x) d x},
\end{aligned}
$$

where $a$ is the semi-axis of the external surface corresponding to the semi-axis $x$. When $\phi(x)$ is given the integrations may be completed; and when $\bar{x}$ is known, the other co-ordinates of the centre of gravity may be inferred from symmetry.

(5) A chord of an ellipse cuts off a segment of constant area; determine the locus of the centre of gravity of the segment.

If a chord cuts off a segment of constant area from a circle, it is evident from the symmetry of the figure that the locus of the centre of gravity of the segment is a concentric circle. Now if the circle be projected orthogonally upon a plane inclined to the plane of the circle the circle projects into an ellipse; and the segments of the circle of constant area project into segments of the ellipse of constant area; also the concentric circle projects into a second ellipse similar to the first ellipse and similarly situated.

Thus the required locus is an ellipse similar to the given ellipse and similarly situated.

This problem might have been solved without making use of projections, in the manner shewn in the next example.

(6) A plane cuts off from an ellipsoid a segment of constant volume; determine the locus of the centre of gravity of the segment. 
Let the cutting plane lase any position; and refer the ellipsoid to coujurate semi-diameters as axes; let the plane of $(y, z)$ be parallel to the position of the cutting plane, and suppose the equation to the ellipsoid to be

$$
\frac{x^{3}}{a^{2}}+\frac{y^{2}}{b^{2}}+\frac{z^{3}}{b^{2}}=1 \text {. }
$$

Now suppose the segment cut off by the plane to be divided into an indefinitely large number of indefinitely thin slices by planes parallel to the plane of $(y, z)$. By the properties of the ellipsoid these slices will be bounded by ellipars which have their centres on the axis of $x$; and thus we see that the centre of gravity of the segment cut off will be on the axis of x. Consider one of the slices bounded by planes which have for their abscissa $x$ and $x+\Delta x$ respectively; then it will be found that the rolume of the slice is ultimately

$$
\pi b \dot{C}\left(1-\frac{x^{2}}{a^{2}}\right) \sin \omega \sin \alpha \Delta x,
$$

where $\omega$ is the angle between the axes of $y$ and $z$, and $\alpha$ is the angle which the axis of $x$ makes with the plane of $(y, z)$. Sujpose $V$ to denote the constant volume, and $\lambda a$ ' the abscissa of the plane cutting off the segment; then

$$
\begin{aligned}
V & =\pi b^{\prime} c^{\prime} \sin \omega \sin \alpha \int_{\lambda \sigma^{\prime}}^{\sigma^{\prime}}\left(1-\frac{x^{2}}{a^{\prime 3}}\right) d x \\
& =\pi a^{\prime} b^{\prime} c^{\prime} \sin \omega \sin \alpha\left(1-\lambda-\frac{1}{3}\left(1-\lambda^{3}\right)\right] .
\end{aligned}
$$

Now by the properties of the ellipsoid

$$
\text { ॠa'b' } c^{\prime} \sin \omega \sin \alpha=\pi a b c,
$$

where $a, b, c$ are the semi-axes of the ellipsoid; thus

$$
V=\pi a b c\left\{1-\lambda-\frac{1}{3}\left(1-\lambda^{3}\right)\right\} \ldots \ldots \ldots \ldots(1) .
$$

And, if $\bar{x}$ be the abscissa of the centre of gravity of the segment cut off, 


$$
\begin{aligned}
\bar{x} & =\frac{\pi b^{\prime} c^{\prime} \sin \omega \sin \alpha \int_{\lambda a^{\prime}}^{a^{\prime}} x\left(1-\frac{x^{2}}{a^{\prime 2}}\right) d x}{V} . \\
& =\frac{\pi a^{\prime 2} b^{\prime} c^{\prime} \sin \omega \sin \alpha}{V}\left\{\frac{1}{2}\left(1-\lambda^{2}\right)-\frac{1}{4}\left(1-\lambda^{4}\right)\right\} \\
& =\frac{\pi a b c}{V}\left\{\frac{1}{2}\left(1-\lambda^{2}\right)-\frac{1}{4}\left(1-\lambda^{4}\right)\right\} a^{\prime} \ldots \ldots \ldots \ldots . .
\end{aligned}
$$

Now (1) gives a constant value for $\lambda$, and then (2) shews that $\bar{x}$ bears a constant ratio to $a$ '.

Thus the locus of the centre of gravity of segments of an ellipsoid of constant volume is an ellipsoid similar to the original ellipsoid and similarly situated.

(7) Find the centre of gravity of a portion of an ellipsoid comprised between two cones whose common vertex is at the centre of the ellipsoid and whose bases are parallel.

The volume between the two cones may be divided into an indefinitely large number of shells which have the centre of the ellipsoid as their common vertex, and their bases in planes parallel to the bases of the two cones. We shall first shew that if the planes which contain the bases of the shells are equidistant the shells are all equal. Take conjugate semidiameters as axes, and let the plane of $(y, z)$ be parallel to the bases of the two cones. The volume of the cone which has the centre of the ellipsoid as vertes, and for its base the plane curve formed by the intersection of the ellipsoid with the plane which has $x$ for its abscissa, is

$$
\frac{1}{3} \pi l^{\prime} c^{\prime} \sin \omega \sin \alpha\left(1-\frac{x^{2}}{a^{\prime 2}}\right) x,
$$

where the notation is the same as in the preceding example. The volume of the cone which has the centre of the ellipsoid as vertex, and for its base the plane curve formed by the intersection of the ellipsoid with the plane which has $x+\Delta x$ for its abscissa, is

$$
\frac{1}{3} \pi b^{\prime} c^{\prime} \sin \omega \sin \alpha\left\{1-\frac{(x+\Delta x)^{2}}{a^{\prime 2}}\right\}(x+\Delta x) .
$$


The wolume of the alice between the planes whose abscismes ans $x$ and $x+\Delta x$ respectirely is ultimalely

$$
\pi{ }^{2} c \sin \omega \sin a\left(1-\frac{x^{2}}{a^{2}}\right) d x \text {. }
$$

Ilence we obtnin for the volume of one of the shells ultimately the product of $\pi$ lid $\sin \alpha \sin \omega$ by

$$
\left[1\left(1-\frac{x^{2}}{a}\right) x+\left(1-\frac{x^{3}}{a^{2}}\right) \Delta x-1\left\{1-\frac{(x+\Delta x)^{2}}{a^{2}}\right\}(x+\Delta x)\right] \text {; }
$$

$1 \mathrm{kis}$ product is ultimately

$$
\frac{2 \pi b^{\prime} c^{\circ} \sin \omega \sin a \Delta x}{3} .
$$

The centse of gravity of earh shell is on the axis of $x$ at a distanes from the vertex of the cone, which is equal to three formtlis of the ateeissa of the plane in which the base of the cone is situated (fee Ex. (0) of Art. 109). Iet $\bar{x}$ denote the alscisea of the ecurre of gravity of the proposed solid; then if $k$ and $k$ te the alscisse of the plane bases of the two concs,

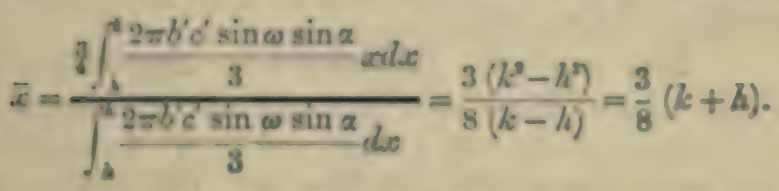

We ahall conelule this Chapter with a few general proi witions involving properties of the centre of gravify.

158. If tha mase of anih of a systom of particles be muliflind into the syuare of its distance from a given point, the sum of the promlaits is least when the given puint is the centre if jrivity of the syotem.

lat the entre of gravity of the system be made the crigin; let $\alpha, \beta, \gamma$, be the cororlinates of the given point; $x_{1}, y_{1}, z_{1}$, the corordinates of the first particle; $x_{2}, y_{2}, z_{v}$ thine of the second; and so on; $m_{1}, m_{0}, \ldots$ the masses of the particles; $\rho_{1}, p_{2}, \ldots$ the distances of the particles from their 
centre of gravity $; r_{1}, r_{2}, \ldots$ the distances of the particles from the fixed point; then

$$
\begin{aligned}
& r_{1}^{2}=\alpha^{2}+\beta^{2}+\gamma^{2}-2\left(x x_{1}+\beta y_{1}+\gamma z_{2}\right)+\rho_{1}^{2}, \\
& r_{3}^{2}=\alpha^{2}+\beta^{2}+\gamma^{2}-2\left(\alpha x_{8}+\beta y_{2}+\gamma z_{2}\right)+\rho_{2}^{2},
\end{aligned}
$$

Multiply these equations by $m_{1}, m_{2}, m_{3}, \ldots$ respectively, and add ; then

$\mathbf{\Sigma} m r^{2}=\left(\alpha^{2}+\beta^{2}+\gamma^{2}\right) \mathbf{\Sigma} m-2(\alpha \mathbf{\Sigma} m x+\beta \Sigma m y+\gamma \mathbf{\Sigma} m z)+\mathbf{\Sigma} m \rho^{2}$. But, since the origin is the centre of gravity of the system,

$$
\Sigma m x=0, \quad \Sigma m y=0, \quad \Sigma m z=0,
$$

therefore $\quad \Sigma m r^{2}=\left(\alpha^{2}+\beta^{2}+\gamma^{2}\right) \Sigma m+\Sigma m \rho^{2}$.

Now $\Sigma m \rho^{2}$ is independent of the position of the given point; hence the least value of $\mathbf{\Sigma} m r^{2}$ is that which it has when $a^{2}+\beta^{2}+\gamma^{2}$ vanishes, that is, when the given point is at the centre of gravity of the system.

139. Let $\alpha_{1}, \beta_{1}, \gamma_{1}$, be the angles which $\rho_{1}$ makes with the axes; $\sigma_{2}, \beta_{2}, \gamma_{3}$, the angles which $\rho_{2}$ makes with the axes; and so on; then we have, supposing the origin the centre of gravity of the system,

$$
\Sigma m \rho \cos \alpha=0, \quad \Sigma m \rho \cos \beta=0, \quad \Sigma m \rho \cos \gamma=0 .
$$

Square each of these equations and add the results; then if $m, m^{\prime}$ represent any two masses, and $\left(\rho, \rho^{\prime}\right)$ the angle between the straight lines which join them with the centre of gravity,

$$
\Sigma m^{2} \rho^{2}+2 \Sigma m m^{\prime} \rho \rho^{\prime} \cos \left(\rho, \rho^{\prime}\right)=0 .
$$

But

$$
2 \rho \rho^{\prime} \cos \left(\rho, \rho^{\prime}\right)=\rho^{2}+\rho^{\prime 2}-u^{2} \text {, }
$$

where $u$ denotes the distance of $m$ and $m^{\prime}$. Hence

$$
\Sigma m^{2} \rho^{2}+\Sigma m m^{\prime}\left(\rho^{2}+\rho^{18}-u^{2}\right)=0 .
$$

If we select the cocfficient of $\rho_{1}^{2}$, we find it to be

$$
m_{1}^{2}+m_{1}\left(m_{2}+m_{3}+\ldots\right) \text {, or } m_{1} \Sigma m,
$$

and the other coefficients are similar. Hence the above equation may be written

$$
\sum m \Sigma m \rho^{2}=\Sigma m m^{\prime} u^{2} .
$$


140. If a particle be arted on by a number of funces ench passing tirowgh a fixed point and proportional to ihe distamse from that point, the resmltant force will pass through a fired point and be proportional to the distance from that point.

Take any position of the particle as the origin; let $x_{1}, y_{0}, z_{2}$, be the co-ordinates of a fixed point; $r_{1}$ the diatance of this point from the erigin; $\mu_{1} r_{t}$ the furee which acts on the particle from this fixed point. Similarly let $x_{2}, y_{3}, z_{s}$, be the co-ordinates of a second fixed point; $r_{2}$ its distance from the origin, and $\mu, r_{i}$ the corresponding force on the particle, and so on. 1, $\boldsymbol{X}, \boldsymbol{Y}, \boldsymbol{Z}$ denote the whele force acting on the particle along the axes of $x, y, z$; then, by Art. 26 ,

$$
\begin{aligned}
X & =\mu_{1} r_{3} \times \frac{x_{1}}{r_{1}}+\mu_{2} r_{3} \times \frac{x_{3}}{r_{1}}+\mu_{8} r_{3} \times \frac{x_{3}}{r_{3}}+\ldots \ldots . \\
& =\mu_{3} x_{3}+\mu_{3} x_{3}+\mu_{2} x_{3}+\ldots . .
\end{aligned}
$$

Similarly $Y=\mu_{1} y_{1}+\mu_{2} y_{1}+\mu_{2} y_{2}+\ldots \ldots$,

and $\quad Z=\mu_{1} 2_{1}+\mu_{2} e_{2}+\mu_{2} 2_{3}+\ldots \ldots$

Let $\bar{x}_{,} \bar{y}_{2} z$ be the co-ordinates of the centre of gravity of a systcm of particles, whose masses are proportional in $\mu_{1}, \mu_{2}, \mu_{3}, \ldots$ placed at the respective fixed points; then

$$
\bar{x}=\frac{\Sigma_{\mu x}}{\Sigma_{\mu}}, \quad \bar{y}=\frac{\Sigma \mu y}{\Sigma \mu}, \quad \bar{z}=\frac{\Sigma \mu z}{\Sigma \mu} ;
$$

therefire $\quad \boldsymbol{X}=\bar{x} \mathbf{v} \mu, \quad Y=\mathbb{y}_{\tilde{\Sigma}} \mu, \quad Z=\bar{z} \mathbf{\Sigma} \mu$.

These cquations shew that the resultant force is equal to ${ }^{\Sigma} \Sigma \mu$, where $r$ is the distance of the centre of gravity from the origin, and that its direction passes through the centre of gravity. Hence when the particle is situated at the centre of grarity the resultant force vanishes and the particle is in equiliturum.

141. A body is placed on a horizontal plane, to find uchen it will be suppoirleal.

The only force acting on it besides the resistance of the plane is its own wright, and this acts in a vertical direction through the centre of gravity of the body. Hence, by 
Art. 91, the body will not be in equilibrium unless the vertical through the centre of gravity of the body falls within a polygon formed by so joining the points of contact of the body and the plane as to include them all and have no re-entering angle.

When a body is suspended from a point round which it can more freely, it will not rest unless its centre of gravity be in the vertical line passing through the point of suspension.

For the body is acted on by two forces, its own weight which acts vertically through its centre of gravity and the force arising from the fixed point ; for equilibrium these forces must act in the same straight line and in opposite directions; thus the centre of gravity must be in the vertical line passing through the point of suspension.

Hence if a body be suspended successively from two points the vertical lines drawn through the points of suspension will both pass through the centre of gravity; therefore the point in which they intersect is the centre of gravity.

If a body be capable of revolving round an axis which is not vertical it will not rest unless the centre of gravity be in the vertical plane passing through the axis. For the body is acted on by its own weight and the forces arising from the fixed points; by Art. 87, the moment of the weight round the fixed axis must vanish, this requires the centre of gravity to be in the vertical plane through the fixed axis.

The student will readily perceive as an experimental fact that there is an important difference between the position of equilibrium in which the centre of gravity is vertically above the fixed point or fixed axis, and that in which it is vertically lelow it. In the former case, if the body be slightly disturbed from its equilibrium position and then left to itself, it will begin to recede from its original position. In the latter case, if the body be slightly disturbed from its equilibrium position and then left to itself, it will begin to return to its original position. 'The former position of equilibrium is called unstable, and the latter stable. We shall return to this point in Chap. XIv. 
142. The volume $(V)$ of a portion of a cylinder intercepted between two planes, one of which is perpendicular to the axis of the cylinder, is given by the equation

$$
\boldsymbol{V}=\iint_{s} d x d y,
$$

where the plane of $(x, y)$ is supposed perpendicular to the axis, and $\mathrm{s}$ is the ordinate of a point in the other plane. The limits of the integrations depend on the curve in which the plane of $(x, y)$ cuts the surface. This follows from the Integral Calculus.

Let $\phi$ denote the angle between the two planes; the aren of an element of the other section of which $\Delta x \Delta y$ is the projection on the plane of $(x, y)$ is $\Delta x \Delta y$ sec $\phi$. Iet $A$ denote the area of the section of the cylinder by the plane of $(x, y)$ and consequently $A$ sec $\phi$ the area of the other section; let : denote the ordinate of the centre of gravity of the plane aren formed by the intersection of the cylinder with the second plano; then

$$
\begin{aligned}
& A \sec \phi \cdot \vec{z}=\iint z \sec \phi d x d y, \\
& \text { or } \quad \\
& \text { therefore } V=\iint z d x d y,
\end{aligned}
$$

The volume is thercfore equal to the area of the base multiflied by the perpendicular upon it from the centre of gravity of the other section.

The centres of gravity of the two plane sections are on the same straight line parallel to the generating lines. For the co-ordinates of the centre of gravity of the section by the plane of $(x, y)$ aro

$$
\iint x d x d y \text { and } \frac{\iint y d x d y}{4} \text {, }
$$

and those of the upper section are

$$
\frac{\int i x \sec \phi d x d y}{A \sec \phi} \text { and } \frac{\int y \sec \phi d x d y}{A \sec \phi},
$$

which agree with the former values.

T. 8 . 
Thus the centres of gravity of all plane sections of a cylinder are situated on a straight line parallel to the generating lines of the cylinder.

If a portion of a cylinder be cut off by two planes, neither of which is perpendicular to the axis, we may suppose it to be the difference of two portions which have for their common base a section perpendicular to the axis. The difference of the straight lines drawn from the centres of gravity of the oblique sections perpendicular to the orthogonal section will be the straight line joining those centres of gravity. Hence the volume of a portion of a cylinder contained between any two planes is equal to the product of the area of an orthogonal section by the straight line joining the centres of gravity of the oblique sections.

143. Through the centre of gravity of each face of a tetrahedron a force acts at right angles to the face, and proportional to the area of the fuce: if the forces all act inwards or all act outwards they will be in equilibrium.

Let $A, B, C, D$ denote the angular points of the tetrahedron. The force acting on the face $A B C$, at its centre of gravity, may be replaced by three equal forces acting at right angles to the face at the points $A, B, C$ respectively. Similar substitutions may be made for the other forces. Thus we have, acting at the point $A$, three forces respectively at right angles to the three faces which meet at $A$ and proportional to the areas of those faces; and, by what has been shewn in the Propositions at the end of Chapter v. these three forces are equivalent to a single force acting at $A$ in the direction perpendicular to the face $B C D$, and proportional to the area of that face. Hence, by Proposition I. at the end of Chapter v, the proposed system of forces will be in equilibrium.

The preceding result may now be extended to the following proposition: Through the centre of graxity of each face of a polyhedron a force acts at right angles to the fuce, and proportional to the area of the face: if the forces all act inwards or all act outucards they will be in equilibrium.

For each face of the polyhedron may be divided into triangles; and the furce, acting at the centre of gravity of 
the face may be replaced by forces acting respectircly at the centres of gravity of the triangles, and proportional in the areas of the triangles. Then the polyhedron may be supposed to be made up of tetrahedrona which have a common vertex, and two equal and opposite forces may be supposed applied at every common face, acting through the centre of gravity of the face at right angles to the face and proportional to the area of the face. Hence the required result follows from the former part of this Article in the manner already exemplified in Proposition 1. at the end of Chapter IV.

The precoding general result was fint brought under the notice of the present writer by the late Bishop Mackenzie; it was given in an examination paper in Gonville and Cains College in 1549, probably by himself. The method by which be demonstrated it will be found interesting and instructive by the student who is acquainted with Hydrostatics. 1 magine a fluid in equilibrium acted on by no forces; then the pressure will be constant throughout the mass. Suppose a purtion of the fluid in the form of a polyhedron to beone solid; then the equilibrium will not be disturbed. The forces acting on the faces of the polyhedron will be respectively at right angles to the faces and proportional to the areas of the faces, and will act through the centres of grarity of the faces. Hence the required result follows.

The proposition may have been enunciated previonsly; hwever a very eminent mathematician stated at the meeting of the Britiali Association at Cheltenham in 1536 , that he havi been unable to find it in print.

By means of Art. 51 we can deduce the following proposiivin respecting couples: A system of couples reprosentid in iwwition and magmitude by the faces of a polyhadrom uill be in equilibium, supposing the ares of the couples all to be finctal incards or all oulucards. This is given by Mübius; Lehrbuch der Stutik, Vol. I. page $\$$. 


\section{Guldinus's Properties.}

144. If any plane figure revolve about an axis lying in ils plane, the content of the solid generated by this figure in revolving through any angle is equal to a prism, of which the base is the revolving figure and height the length of the path described by the centre of gravity of the area of the plane figure.

The axis of revolution in this and the following proposition is supposed not to cut the generating curve.

Let the axis of revolution be the axis of $x$, and the plane of the revolving figure in its initial position the plane of $(x, y)$; let $\beta$ be the angle through which the figure revolves.

The elementary area $\Delta x \Delta y$ of the plane figure in revolving through an angle $\Delta \theta$ generates the elementary solid whose volume is $y \Delta \theta \Delta x \Delta y$; therefore the whole solid

$$
=\iiint_{0}^{\beta} y d x d y d \theta=\beta \iint y d x d y \text {. }
$$

The limits of $x$ and $y$ depend on the nature of the curve. But if $y$ be the ordinate to the centre of gravity of the plane figure, then, by Art. 118,

$$
\bar{y}=\frac{\iint y d x d y}{\iint d x d y},
$$

the limits being the same as before.

Therefore the whole solid $=\beta \iint y d x d y=\bar{y} \beta \iint d x d y=$ the arc described by the centre of gravity multiplied by the area of the figure.

If any figure revolve about an axis lying in its own plane, the surface of the solid generated is equal in area to the rectangle, of which the sides are the length of the perimeter of the generating figure and the length of the path of the centre of gravity of the perimeter.

The surface generated by the arc $\Delta s$ of the figure revolving through an angle $\Delta \theta$ is $y \Delta \theta \Delta s$; therefore the whole surface

$$
=\iint_{0}^{\beta} y d s d \theta=\beta \int y d s \text {. }
$$


The limits depend on the nature of the curve. But if $\bar{y}$ be the ordinate to the centre of gravity of the perimeter,

$$
\bar{y}=\int \frac{\int y d s}{\int d x},
$$

the linits being the same as before.

Therefore the whole surfuce $=$ i, $\beta \int d=$ the are described by the centre of grarity, multiplied by the length of the perimeter.

Fix. 1. To find the solid content and the surface of the ring formal by the recolution of a circle round a straight line in its uon plane which it does not meet.

Lat the distance of the centre of the circle from the axis of revolution be a; let $b$ be the radius of the circle; then the length of the path of the centre of gravity of the area of the figure is $2 \pi u$, and the area of the figure is $\pi b^{2}$;

therefure the content of the solid $=2 \pi^{2} a b^{2}$.

Also the length of the path of the centre of gravity of the perimeter is $2 \pi a$, and the length of the perimeter is $2 \pi b$;

therefore the surface of the solid $=4 \pi^{2} a b$.

Fx. 2. To find the centre of gravity of the area and ulso of the are of a semicircle.

A semicircle by revolving about its diameter generates a sphere; the content of the sphere is $\frac{4}{3} \pi a^{3}$, and the surface $4 \pi \omega^{2}$, the radius being $a$; the area of the semicircle is $\frac{1}{2} \pi a^{2}$, and the perimeter $\pi a$; therefore, the distance of the centre of gravity of the area from the diameter

$$
=\frac{\text { content of sphere }}{2 \pi \cdot \operatorname{arca} \text { of semicircle }}=\frac{1 a}{3 \pi} \text {; }
$$

the distance of the centre of gravity of the are from the diameter

$$
=\frac{\text { surface of sphenc }}{2 \pi . \text { are of semicircle }}=\frac{2 a}{\pi} \text {. }
$$


Ex. 3. To find the surface and the solid content of the solid formed by the revolution of a cycloid round the tangent at its vertex.

In $\Lambda$ rt. 133 we have found $\frac{2 a}{3}$ for the distance of the centre of gravity of the arc of a cycloid from its vertex; and the whole length of the arc is $8 a$. Therefore the surface of the solid generated is

$$
2 \pi \times \frac{2 a}{3} \times 8 a \text {; that is } \frac{32}{3} \pi a^{2} .
$$

And in Art. 113 we have found that the distance of the centre of gravity of the area included between the cycloid and its base from the vertex is $\frac{7}{6} a$; and the area 80 included is $3 \pi a^{2}$. Hence the area of the portion which in the present case revolves round the tangent is $4 \pi a^{2}-3 \pi a^{2}$, that is $\pi a^{2}$. And the centre of gravity of this area may be shewn to be at a distance $\frac{a}{2}$ from the vertex. (See Ex. (2) of Art. 109.) Therefore the solid content of the figure generated is $2 \pi \frac{a}{2} \pi a^{2}$, that is $\pi r^{2} a^{3}$.

\section{EXAMPLES.}

1. Find the centre of gravity of five equal heavy particles placed at five of the angular points of a regular hexagon.

2. Five pieces of a uniform chain are hung at equidistant points along a rigid rod without weight, and their lower ends are in a straight line passing through one end of the rod; find the centre of gravity of the system.

3. A plane quadrilateral $A B C D$ is bisected by the diagonal $A C$, and the other diagonal divides $A C$ into two parts in the ratio of $p$ to $q$; shew that the centre of gravity of the quadrilateral lies in $A C$ and divides it into two parts in the ratio of $2 p+q$ to $p+2 q$. 
4. From the fact that any system of heavy particles has nue centre of gravity and ouly one, deduce the property that the straight lines foining the middle points of the opposite siles of any quadrilateral figure bisect each other.

5. A pyramid stands on a square base: given the co-ordimates of the vertex, and the co-ordinates of two opposite corners of the base, determine the co-ordinates of the centre of gravity of the pyramid.

6. $A B C$ is a triangle; $D, E, F$ are the middle points of its sides; shew that the centre of gravity of the sides of $A B C$ coincides with the centre of the circle inscribed in DEF.

7. A piece of wire is formed into a triangle; find the distance of the centre of gravity from each of the sides, and ahew that if $x, y, z$ be the three distances, and $r$ the radius of the inseribed circle, then

$$
\text { tryz }-r^{2}(x+y+z)-r^{2}=0 .
$$

8. If the centre of gravity of a four-sided figure coincide with one of its angular points, shew that the distances of this point and the opposite angular point from the straight line joining the other two angular points are as 1 to 2 .

9. Shew that the common centre of gravity of a rightangled isosceles triangle, and the squares described on the two equal sides, is at a distance $=\frac{\sqrt{2}}{15} a$ from the point in which those sides meet, $a$ being the length of one of them.

10. Prove the following construction for the centre of gravity of any quadrilateral. Iet $E$ be the intersection of the diagonals, and $F$ the midtle point of the straight line. which joins their middle points: draw the straight line $E F^{\circ}$ and produce it to $\vec{G}$, making $F G=J E F$; then $G$ shall be the centre of gravity required.

11. A triangle $A B C$ is successively suspended from the angles $A$ and $B$, and the two positions of any sile are at right angles to each other; shew that

$$
b c^{2}=a^{2}+b^{2}
$$


12. A right-angled triangular lamina $A B C$ is suspended from a point $D$ in its hypothenuse $A B$; prove that in the position of equilibrium $A B$ will be horizontal if

$$
A D: D B:: A B^{2}+A C^{n}: A B^{2}+B C^{2} \text {. }
$$

13. $\Lambda$ given isosceles triangle is inscribed in a circle; find the centre of gravity of the remaining area of the circle.

14. If three uniform rods be rigidly united 80 as to form half of a regular hexagon, prove that if suspended from one of the angles, one of the rods will be horizontal.

15. If $A B C$ be an isosceles triangle having a right angle at $C$, and $D, E$ be the middle points of $A C, A B$ respectively, prove that a perpendicular from $E$ upon $B D$ will pass through the centre of gravity of the triangle $B D C$.

16. $A B C D$ is any plane quadrilateral figure, and $a, b, c, d$ are respectively the centres of gravity of the triangles $B C D$, $C D A, D A B, A B C$; shew that the quadrilateral abcd is similar to $A B C D$.

17. $A, B, C, D, E, F$ are six equal particles at the angles of any plane hexagon, and $a, b, c, d, e, f$ are the centres of gravity respectively of $A B C, B C D, C D E, D E F, E F A$, and $F A B$. Shew that the opposite sides and angles of the hexagon abcdef are equal, and that the straight lines joining opposite angles pass through one point, which is the centre of gravity of the particles $A, B, C, D, E, F$.

18. A straight line $E D$ cuts off $\frac{1}{n}$ th part of the rightangled triangle $A B C$ of which $A$ is the right angle. $A B=a$, $A C=b$. Shew that the centre of gravity of $C E D B$ describes the curve whose equation is

$$
\frac{a b}{n}=\{3(n-1) y-n b\}\{3(n-1) x-n a\} .
$$

19. The distance of the centre of gravity of any number of sides $A B, B C, C D \ldots \ldots K L$ of a regular polygon from the centre of the inscribed circle

$$
=\frac{A L \times \text { radius }}{A B+B C+C D+\ldots . . .+K L} .
$$


20. $\mathbf{A}$ frustum is cut from a right cone by a plane bisecting the axis and parallel to the base; shew that it will rest with its slant side on a horizontal table if the height of the cone bear to the diameter of its base a greater ratio than $\sqrt{7}$ to $\sqrt{17}$.

21. If particles of unequal weights be placed at the angular points of a triangular pyramid, and $G_{1}$, be their common centre of gravity; $G_{0}, G_{3}, \ldots$ their common centres of gravity for every posaibile arrangement of the particles; shew that the centre of gravity of equal particles placed at $G_{1}, G_{2}, \ldots$ is the centre of gravity of the pyramid.

22. If a cote have its base united concentrically to the base of a hemisphere of equal radius, find the height of the cone that the solid may rest on a horizontal tatile on any point of its spherical surface.

liesule. $r \sqrt{ } 3$.

23. If any polygon circumscribe a circle, the centre of gravity of the area of the polygon, the centre of gravity of the perimeter of the polygon, and the centre of the circle, are in the same straight line; also the diatance of the first point from the third is two-thirds of the distance of the second point from the third.

24. If any polyhedron circumscribe a sphere, the centre of gravity of the volume of the polyhedron, the centre of gravity of the surface of the polyhedron, and the centre of the sphere, are in the same straight line; also the distance of the first point from the third is three-fourths of the distance of the second point from the third.

25. From a right cone the diameter of whose base is equal to its altitude is cut a right cylinder the diameter of whose bnse is eyual to its altitude, their axes being in the same straight line and the base of the cylinder lying in the base of the cone; from the remaining cone a similar cylinder is cut, and so on, indefinitely; shew that the distance of the centre of gravity of all the eylinders frum the bnse of the cone is $\frac{3}{10}$ of the height of the cone, and that the distance of the centre of gravity of the remaining portion from the base of the cone is 15 of the altitude of the cone. 
26. A square is cut from an equilateral triangle, a side of the square coinciding with a side of the triangle; from the equilateral triangle which remains another square is cut, and so on, ad infinitum: find the centre of gravity of the sum of the squares.

27. Find the centre of gravity of the area contained between the curves $y^{2}=a x$ and $y^{z}=2 a x-x^{2}$, which is above the axis of $x$.

$$
\text { Results. } \bar{x}=a \cdot \frac{15 \pi-44}{15 \pi-40} ; \bar{y}=\frac{a}{3 \pi-8} \text {. }
$$

28. Find the centre of gravity of the area enclosed by the curve $r=a(1+\cos \theta)$.

Result. $\bar{x}=\hat{\mathrm{g}} a$.

29. Find the centre of gravity of the area included by a loop of the curve $r=a \cos 2 \theta$.

$$
\text { Result. } \bar{x}=\frac{128 a \sqrt{ } 2}{105 \pi} .
$$

30. Find the centre of gravity of the area included by a loop of the curve $r=a \cos 3 \theta$.

Result. $\bar{x}=\frac{81 \sqrt{ } 3 a}{80 \pi}$.

31. The locus of the centre of gravity of all equal segments cut off from a parabola is an equal parabola.

32. Find the centre of gravity of a segment of a circle.

33. Find the centre of gravity of the area included by the curves $y^{2}=a x$ and $x^{2}=b y$.

$$
\text { Result. } \bar{x}=\frac{9}{20} a^{\frac{3}{3}} b^{\frac{2}{3}}, \bar{y}=\frac{9}{20} a^{\frac{3}{3}} b^{\frac{1}{3}} \text {. }
$$

34. Find the centre of gravity of a portion of an equilateral hyperbola bounded by the curve, the transverse axis, and a radius vector drawn from the centre.

$$
\text { Results. } \begin{aligned}
\bar{x} & =\frac{2 y^{\prime}}{3 \log \left(x^{\prime}+y^{\prime}\right)-3 \log a} ; \\
\bar{y} & =\frac{2\left(x^{\prime}-a\right)}{3 \log \left(x^{\prime}+y\right)-3 \log a}
\end{aligned}
$$

where $x^{\prime}, y^{\prime}$ are the co-ordinates of the point of intersection of the curve and the bounding radius vector. 
35. Two equal cireles of radius a are drawn, each panaing through the centre of the other, and a thind circle touches lroth, laving one of their points of intersection for its centre; the distance of the centre of gravity of the smaller area included between the outer and inner circles from the common radius of the first two is

$$
\begin{aligned}
& 12-2 \pi \sqrt{3} \\
& 2 \pi-3 \sqrt{3}
\end{aligned}
$$

38. The denaity of a triangle varies as the $n^{\mathrm{m}}$ power of the distance from the base; determine $n$ when the centre of gravity of the triangle divides the straight line joining the vertex with the middle point of the base in the ratio of 3 to 1 . Reault, $\mathrm{n}=-\mathrm{f}$.

37. Find the centre of gravity of the volume formed by the revolntion round the axis of $x$ of the area of the curve $y^{6}-a x y^{3}+x^{6}=0$. Resull. $\bar{x}=\frac{3 a \pi}{32}$.

38. Find the centre of gravity of the volume generated by the revolution of the area in Ex. 27 round the axis of $y$.

$$
\text { Result. } \bar{y}=\frac{5 a}{2(15 \pi-44)} \text {. }
$$

39. Find the centre of gravity of a hemisphere when the deusity varies as the square of the distance from the centre.

$$
\text { Resule. } \bar{x}=\frac{5 a}{12} \text {. }
$$

40. Find the centre of gravity of the solid generated by a semiparabola bounded by the latus rectum revolving round the latus rectum.

Resule. Distance from focus $=3$ of latus rectum.

41. The solid included between the surfaces of a onntinuous hyperboloid and its conical asympeote is cut by two planes perpendicular to their common axis; find the position of the centre of gravity of that portion which lies between the planes.

Resulb. Midway between the planes. 
42. $\Lambda$ solid sector of a sphere hangs from a point in its circular rim with its axis horizontal, find its vertical angle.

Result. The cosine of the semi-vertical angle is $\frac{?}{6}$.

43. Find the centre of gravity of the solid generated by the revolution of a semicircle about a straight line perpendicular to the diameter, and which does not meet the semicircle.

Result. Distance from the plane generated by the diameter

$$
=\frac{4 r}{3 \pi} \text {. }
$$

44. $A$ is a point in the generating line of a right cylinder on a circular base, and $B, C$ are two others in the generating line diametrically opposite. The cylinder is bisected by a plane $A B C$, and one of the semicylinders is cut by two planes at right angles to $A B C$, passing through $A B$ and $A C$. Shew that if the solid $A B C$ be placed with its convex side on a horizontal plane, the plane $A B C$ will be inclined to the horizon at an angle $\tan ^{-1}\left(\frac{3}{10} \pi\right)$, when there is equilibrium.

45. A solid cone is cut by two planes perpendicular to the same principal section, one through its axis, and the other parallel to a slant side; find the limiting value of the vertical angle of the cone, that the piece cut out may rest on its curved surface on a horizontal plane.

Result. The cosine of the vertical angle must not be greater than $\frac{k}{y}$.

46. A quadrant of a circle revolves round one of its extreme radii through an angle of $30^{\circ}$; find the centre of gravity of the solid traced out, the density being supposed to vary as the distance from the centre.

Results. $\bar{x}=\frac{3 a}{5} ; \bar{y}=\frac{3 a}{5}(2-\sqrt{3}) ; \bar{z}=\frac{2 a}{5}$. The axis of $x$ is supposed to coincide with the initial position of the revolving radius. 
47. A solid is formed by the revolution of the amen of the curve $y^{s-1}=a x^{p-0}$ round the axin of $x$; whew that the fis.

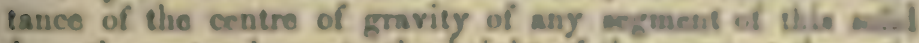
from the vertex bears to the height of the merment the nata of 1 to $\mathrm{n}$. The segment is supposed cut off by a plasse pers pendicular to the axis.

48. Find the centre of gravity of the surface of the nothid $z^{0}+y^{2}=2 a x$, cut off by the plane $z=c$

$$
\text { Result. } \bar{z}=\frac{(2 c-a)(a+2 c \mid+a !}{8 \mid(a+2 c) !-d]} \text {. }
$$

49. Apply Guldinus's thenrem to find the volume of the frustum of a right cone in termai of its altitule and the malu of its exads.

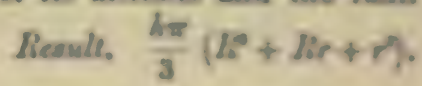

50. Find the aurface and the rolume of the colld formed by the revolution of a cycloid round is bace.

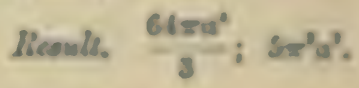

51. A segment of a circle revolves round ita chant, wheh subtends an angle of $90^{\circ}$ at the centre; find the surfuce and volume of the solid generated.

$$
\text { Resules. } \frac{m^{2}(t-\pi)}{\sqrt{2}} ; \frac{a^{2}(10-3 t)}{6 \sqrt{2}} \text {. }
$$

52. An ellipso whoee excentricity in $\frac{4}{8 \pi}$ revolve alsows any tangent line. Prove that the volume generatell by cse prerion into which the ellipse in divided by les mibed asis varies inventely as the volume generated by the other porrices.

53. A plane arca moves in auch a masuer at to in almay normal to the curve along which its center of grovily moves prove that the volume generated is gyual to the firme asere inultiplied by the length of the path of the erntre ef gravity.

Ilence find the rolume of a cycloidal tabe whoe nuesal section is of constant area. 
54. Extend Guldinus's theorem for finding the volume of a ring to the case in which the ring is formed by the revolution of a plane area about a straight line parallel to its plane.

A ring is formed by the revolution of the lemniscate (whose equation is $r^{2}=a^{8} \cos 2 \theta$ ) about a straight line parallel to its plane situated in a plane drawn through its double point and perpendicular to its axis; shew that the volume of this ring is $\frac{\pi^{2} a^{3}}{4 \sqrt{2}}$. 


\title{
(175)
}

\section{CHAPTER IX.}

\author{
Macilssiato
}

145. A Macurse is an instrument, of a pystem of a III byelies, for the purpose of tratsatnitting furce frum owe part the another of the syatem.

It would te endlies to dencrite all the enachities thas have

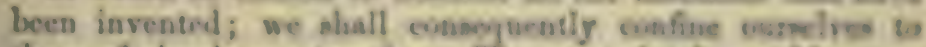
those of simple construction. The mist simple mats.es aso denominated the Mechanteal Towern. Theoe we phall esplain, and also a few combinations of them.

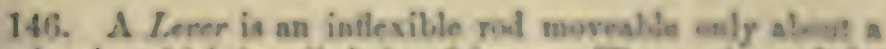
fixed axis, which is called the fulcrum. The jertions of the lever inte whish the fulerum divides it are called tle oros of the lever: when the arme are in the same straight lines it is called a streight lecer, and in other cones a teont heer.

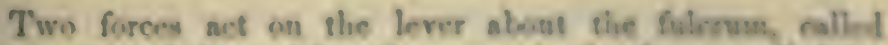

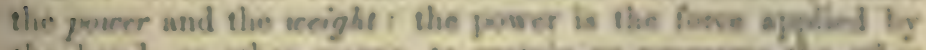
the hand, of other nobss, 60 suseain or overoomen the elles

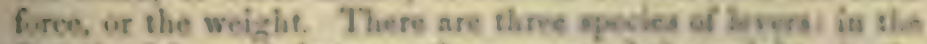
first the fulcrum is letween the power fed the origats is the scound the weight acts betwoen the fakruse and the porve: and in the thirl the power acts between the falcrum ased tho weight.

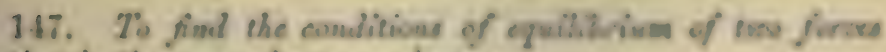
acting in the sume plane on a lecer. 
Let the plane of the paper be the plane in which the forces act, and also be perpendicular to the axis, of which $C$ is the projection, and about which the lever can move; $A, B$ the points of application of the forces $P, W ; \alpha, \beta$ the angles which the directions of the forces make with any straight line $a \mathrm{Cb}$ drawn through $C$ on the paper. Let $R$ be the pres-

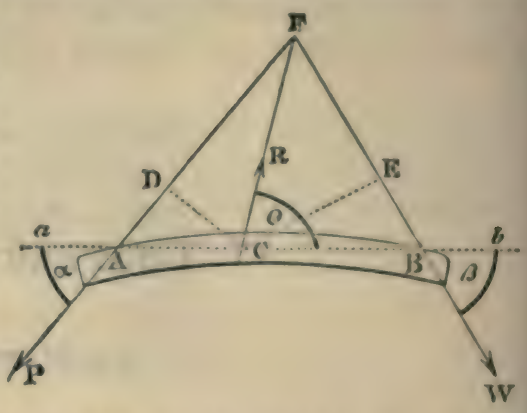
sure on the fulcrum, and $\theta$ the angle which it makes with the straight line $a \mathrm{Cb}$; then if we apply a force $R$ in the direction $C R$, we may suppose the fulcrum removed, and the body to be held in equilibrium by the forces $P, W, R$.

We shall resolve these forces in directions parallel and perpendicular to $a C b$; and also take their moments about $C$; then by Art. 57 we have the following equations:

$$
\begin{aligned}
P \cos \alpha-W \cos \beta-R \cos \theta & =0 \ldots \ldots \ldots \ldots(1), \\
P \sin \alpha+W \sin \beta-R \sin \theta & =0 \ldots \ldots \ldots \ldots(2), \\
P . C D-W . C E & =0 \ldots \ldots \ldots \ldots(3),
\end{aligned}
$$

and

$C D$ and $C E$ being drawn perpendicular to the directions of $P$ and $W$.

These three equations determine the ratio of $P$ to $W$ when there is equilibrium; and the magnitude and direction of the pressure on the fulcrum.

For equation (3) gives

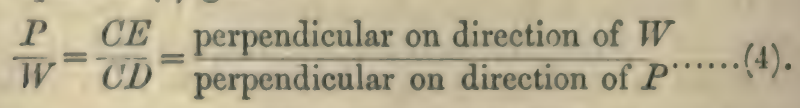

Also by transposing the last terms of (1) and (2), we have

$$
\begin{aligned}
& R \cos \theta=P \cos \alpha-W \cos \beta, \\
& R \sin \theta=P \sin \alpha+W \sin \beta .
\end{aligned}
$$


Ad their squares ; therefore

$$
I^{\circ}=X^{m}+W^{\prime \prime}-2 W^{\prime} \cos \left(x+\beta^{3}\right)
$$

which gives the magnitude of $\mathbb{R}$.

From (I) and (2) by tranopositian and divioion

$$
\tan \theta=\frac{P \sin \alpha+W \sin \beta}{P \cos a-W \cos \beta},
$$

which gives the direction of the presure.

If we suppose $B$ is le the fulcram and tale the roomenge strout $n$ insteal of $C$, we have instead of eqgation (4) the following:

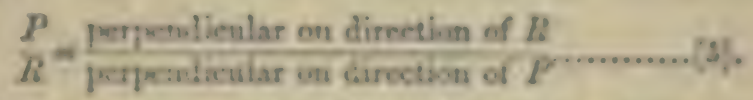

This is not a sew epuatiog of ondition; bat is a caneo

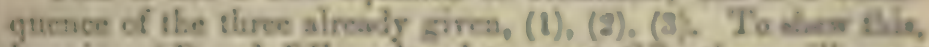

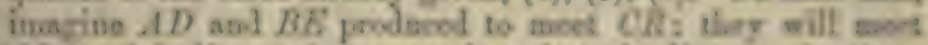
this straight line at the name point, wines the distarms by dese two constructives are $C D$ cowe $(\theta-a)$ and $C B$ enere $(\theta+A)$; and these are made equal, by equations (1), $(2),(9)$, if s: eliminate $P$ and $T$. Suppose, then, Foo be the pout at whels these straight lines meet. By multiplying (1), P, sejpoc lively by $\sin \beta$ and cos $\beta$, and adding, we have

$$
\begin{aligned}
& \frac{P}{R}=\frac{\sin (\theta+B)}{\sin (a+B)}=\frac{W \sin (\theta+B)}{W \sin (a+B)}
\end{aligned}
$$

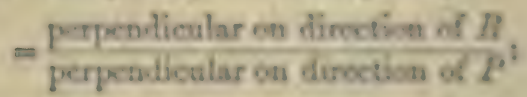

therciore this equation is a consoguence of the cyra:iems (1), (2), (3), as might hase boen asticipated

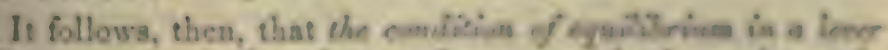

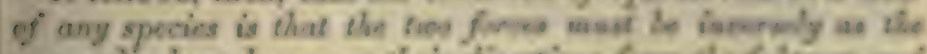

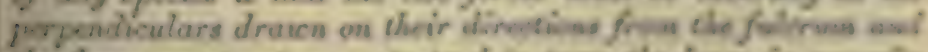

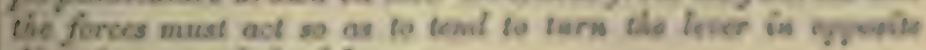
dircetions rousul the fuloruan.

149. This property of the heres molers is a weint bo strument in multiplying the chlowey of a fortes Vor ang tmo 
forces, however unequal in magnitude, may be made to balance each other simply by fixing the fulcrum so that the ratio of its distances from the directions of the forces shall be equal to the inverse ratio of the forces. If the fulcrum be moved from this position, then that force will preponderate from which the fulcrum is moved and the equilibrium will be destroyed. We are thus led to understand how mechanical advantage is gained by using a crow-bar to move heavy bodies, as large blocks of stone: a poker to raise the coals in a grate: scissors, shears, nippers, and pincers; these last consisting of two levers of the first kind. The brake of a pump is a lever of the first kind. In the Stanhope printing-press we have a remarkable illustration of the mechanical advantage that can be gained by levers. The frame-work in which the paper to be printed is fixed, is acted on by the shorter arm of a lever, the other arm being connected with a second lever, the longer arm of which is worked by the pressman. These levers are so adjusted that at the instant the paper comes in contact with the types, the perpendiculars from the fulcra on the directions of the forces acting at the shorter arms are exceedingly short, and consequently the levers multiply the force exerted by the pressman to an enormous extent.

As examples of levers of the second kind, we may mention a wheelbarrow, an oar, a chipping-knife, a pair of nutcrackers.

It must be observed, however, that as the lever moves about the fulcrum the space through which the weight is moved is, in the first and second species of lever, smaller than the space passed through by the power: and therefore what is gained in power is lost in despatch. For example in the case of the crow-bar: to raise a block of stone through a given space by applying the hand at the further extremity of the lever, we must move the hand through a greater space than that which the weight describes.

But in the third species of lever the reverse is the case The power is nearer the fulcrum than the weight, and is con sequently greater; but the motion of the weight is greate than that of the power. In this kind of lever despatch i gained at the expense of power. $\Lambda$ n excellent example $i$ the treddle of a turning lathe. But the most striking ex 
ample of levers of the third kind in found in the anima! fraene. in the construction of which it neetus to be a pervailing pecter-

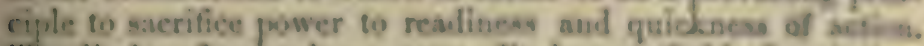
The limbs of amimals are generally levers of this deserspelou. The conilgle of the bone rests in its socket an the fulersas is a strong muscle aftached to rise bone near the condyle is the power, and the weight of the limb together with any no sistance opposerl to its invion is the weight. A sligtat comsraction of che muscle gives a considerable motion to the limb.

149. The lever is applied to determine the weight of sulstances. Under elies character it is called a Balanes. The I. monom Balonce has its two arme equal, with a male nes perulal from each extremity; tha fulcrum leusg alere the contre of gravity of the heam and thereioge above the ersire of gravity of the system formed by the heom, tae males, and the weiches in the scales. The substanes of lo welghid is placol in one scale, and seights placest is the ctivg sill the fenm remains in equilibrium in a perlectly horizosal pesi-

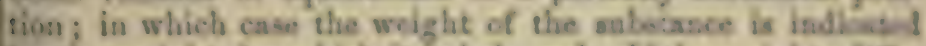
Iy the wrighes by which it is balanced. If the weleles diEs? ever so stighily the horizontality of the beam will be dis. iuried, and after caclllaring for some time, in conacyanese of iive fulcrum being placed alsee the centre of fraviry of the pjotem, it will, on actaining a state of rest, forsa as angles With the horizon, the extent of which is a meavure al tic ingibility of the balanoe.

Whin we take the weight in the other scale as a mesomm of the weight of the subatance we are weighing, we asousas thas the arms of the lever are of equal lezuth and that the lowa

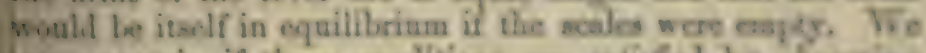

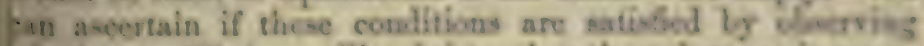

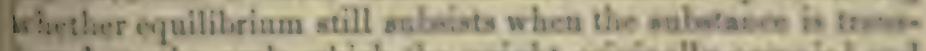

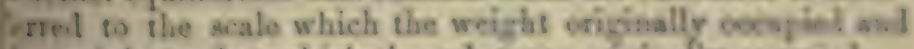
in weight to that which the substance originally exwioch.

150. In the conseruction of a lablance the fillowing so givites should be attended to.

(1) When loaded with orgal weights the heam ahvaht le serfectly horizontal.

2) When the weigles differ, cven ly a slfeth guantity.

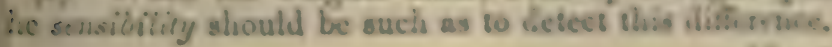


(3) When the balance is disturbed it should readily return to its state of rest, or it should have stability.

We shall now consider how these requisites may be satisfied To find how the requisites of a good lialance may be satisficd Let $P$ and $Q$ be the weights in the scales; let $A B=2 a$

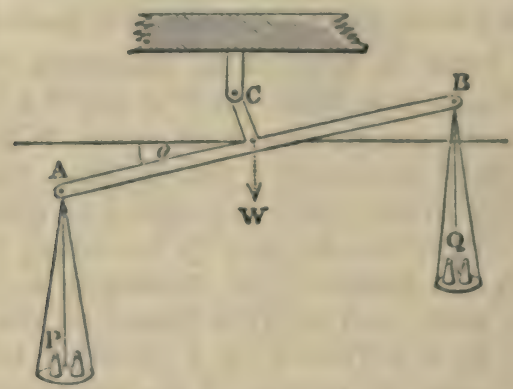

Let $C$ be the fulcrum, $h$ its distance from the straight lin which joins $A$ and $B$. Let $W$ be the weight of the bearn 7 the distance of the centre of gravity of the beam from $C$ this centre of gravity being supposed to lie on the perpen dicular from $C$ on the straight line which joins $A$ and $B$ Let $S$ be the weight of each scale; so that $P$ and $S$ ac vertically through $A$, and $Q$ and $S$ vertically through $B$ Iet $\theta$ he the angle which the beam makes with the horizor when there is equilibrium.

'The sum of the moments of the weights round $C$ will br zero when there is equilibrium, by Art. 57. Now the lengtl of the perpendicular from $C$ on the line of action of $P$ and is $a \cos \theta-h \sin \theta$; the length of the perpendicular from ( on the line of action of $Q$ and $S$ is $a \cos \theta+h \sin \theta$; and thi length of the perpendicular from $C$ on the line of action of 11 is $l_{i} \sin \theta$. Therefore

$(Q+S)(a \cos \theta+h \sin \theta)-\left(P+S^{\prime}\right)(a \cos \theta-h \sin \theta)+W \% \sin \theta=0$

therefore

$$
\tan \theta=\frac{(P-Q) a}{\left(P+Q+2 S^{\circ}\right) h+W k} .
$$


This deternines the position of equilibriam. The fors reguisite the borizontality when $D^{D}$ and $Q$ ape equal-is satisited ly making the arms equat.

For the scoond we obscrve that for a given ditirnenes of $P$ anil $Q$ the sensibility is greater the greater tas $\theta$ is i an? fer a given valie of $\tan \theta$ the sensibility is greater tiee subller the difference of $P$ and $Q$ is : hence $\frac{\tan \theta}{P-Q}$ is a correc mesame of the sensibility: and therefore the moond ropulaite is fabfilled by making $(P+Q+2 S) \frac{h}{a}+W^{\frac{k}{a}}$ an mall a powille.

The statility is greater the greater the moment of the fores which fend to restore the Gquilibrium when it is be stroyed. Now this mortent is

$$
([P+Q+2 S\rangle h+W\}) \text { ain } \theta-\langle P-Q] \text { a cose }
$$

cr supposing $P$ and $Q$ equal it is

$$
\{(P+Q+2 S] h+W\}] \sin \theta .
$$

Ilence to matisfy the thind reguinite, this mest be moth as Alarge as possible. This is, in part, at rariasece witl ile

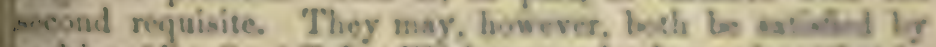
making $(P+Q+2 S) h+W h$ large, and a large alos: that be, by increasing the diatances of the fulerum fruen tise lessen Find from the centre of gravity of the beam, asul by lenghlenang the arms.

It unat be remarked that the sensibility of a balanse is of m.re importance than the stability, nimoe the ege can jolys I wetily accurately whether the index of the beasm makes ofsal If collations on each side of the vercieal lines that is, whesters the position of rest woald be horizontals if this be set the ase, then the weights must be altered till the cuctlatinus ane karly equal.

151. Another kind of balance is that in which ti.e arma

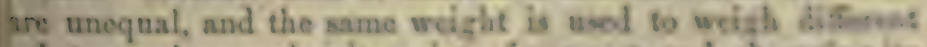
ahstances by varying lis point of mofport, and flemine is Tistance from the fulerum by means of a graduated sesils. The common stoelgand is of this description. 
152. To shew how to graduate the common steelyard.

Let $A B$ be the beam of the steelyard. $A$ the fixed point

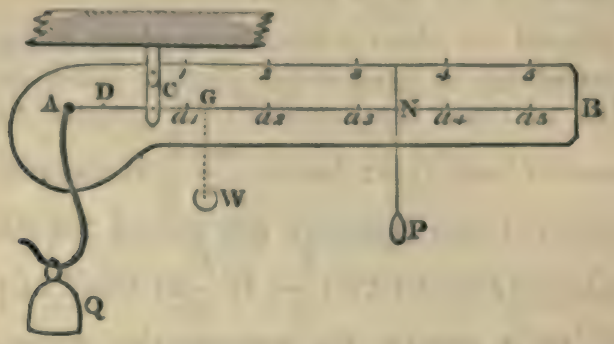

from which the substance to be weighed is suspended, $Q$ being its weight; $C$ the fulcrum; $W$ the weight of the beam together with the hook or scale-pan suspended from $A ; G$ the centre of gravity of these.

Suppose that $P$ suspended at $N$ balances $Q$ suspended from $A$; then, taking the moments of $P, Q$, and $W$ about $C$, we have

$$
\begin{gathered}
Q \cdot A C-W \cdot C G-P \cdot C N=0 \\
Q=\frac{C N+\frac{W}{P} \cdot C G}{A C} P .
\end{gathered}
$$

Take the point $D$, so that $C D=\frac{W}{P} C G$; therefore

$$
Q=\frac{C N+C D}{A C} P=\frac{D N}{A C} P .
$$

Now let the arm $D B$ be graduated by taking $D a_{1}, D a_{2}$, $D a_{3}, \ldots \ldots$ equal respectively to $A C, 2 A C, 3 A C \ldots \ldots$; let the figures $1,2,3,4, \ldots \ldots$ be placed over the points of graduation, and let subdivisions be made between these. Then by observing the graduation at $N$ we know the ratio of $Q$ to $P$; and $P$ being a given weight we know the weight of $Q$. In this way any substance may be weighed.

153. The second of the Mechanical Powers is the Wheel and Axle. 'This machine consists of two cylinders fixed 
together with their axes in the same straight line: the larger cylinder is colled the wheel, and the smaller the axle. The cord by which the weight is suspended is fastened to the axle, and then coiled round it, while the power which supports the weight acts by a cord coiled round the circumference of the wheel, by apokes acted on by the hand, as in the sapstem, or by the hand acting on a handle, as in the woindluss.

154. To find the ratio of the pover and weight in the Wheel and Axlo when in equilibrium.

Iet $A D$ be the wheel and $C C^{\prime} B$ the axle; $P$ the power represented by a weight suspended frum the circumference of the wheel at $A ; W$ the weight hanging from the axle at $B$.

Then since the axis of the machine is fixed, the condition of equilibrium is that the sum of the moments of the forres about this axis vanishes, (Art. 87); therefore

$P \times$ rad. of wheel $=W \times$ rad. of axle;

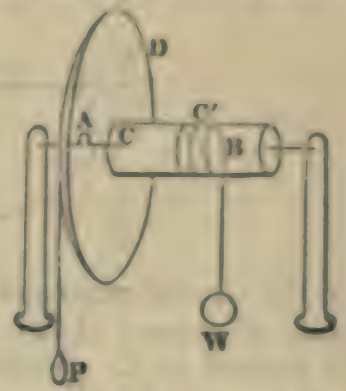

therefore $\frac{W}{P^{\prime}}=\frac{\text { rad. of wheel }}{\text { rad. of asle }}$.

It will be seen that this machine is only a modification of the lever. In short it is an assemblage of levers all having the same axis: and as soon as one lever has been in action the next comes into play; and in this way an endless levernge is obtained. In this respect, then, the wheel and axle surpasses the common lever in mechanical advantage. It is much used in docks and in shipping.

155. The third Mechanical Power is the Toothed Wheel. It is extensively applied in all machinery; in cranes, steamengines, and particularly in clock and wateh work. If two circular hoops of metal or wood having their outer circumferences indented, or cut into equal teeth all the way round, be so placed that their edges touch, one tooth of one circum- 
ference lying between two of the other (as represented in the figure); then if one of them be turned round by any menns, the other will be turned round also. This is the simple construction of a pair of toothed whecls.

156. To find the relation of the power and weight in Toothed Wheels.

Let $A$ and $B$ be the fixed centres of the toothed wheels

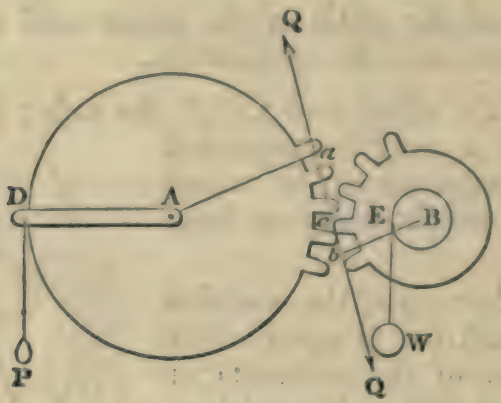

on the circumferences of which the teeth are arranged; $C$ the point of contact of two teeth; $Q C Q$ a normal to the surfaces in contact at $C$. Suppose an axle is fixed on the wheel $B$, and the weight $W$ suspended from it at $E$ by a cord; also suppose the power $P$ acts by an arm $A D$; draw $A a, B b$ perpendicular to $Q C Q$. Let the mutual pressure at $C$ be $Q$. Then, since the wheel $A$ is in equilibrium about the fixed axis $A$, the sum of the moments about $A$ equals zero; therefore

$$
P \cdot A D-Q \cdot A a=0 .
$$

$A$ so since the wheel $B$ is in equilibrium about $B$, the sum of the moments about $B$ equals zero; therefore

$$
Q \cdot B b-W \cdot B E=0 .
$$

Then by eliminating $Q$ from these two equations,

$$
\begin{aligned}
& \frac{P}{W}=\frac{P}{Q} \cdot \frac{Q}{W}=\frac{A a}{A D} \cdot \frac{B E}{B b} ; \\
& \text { or } \frac{\text { moment of } P}{\text { moment of } I V}=\frac{A a}{B b}:
\end{aligned}
$$


when the techs are small this ratio very nearly

$$
=\frac{\text { rad. of wheel } A}{\text { rad. of wheel } B} \text {. }
$$

157. Wheels are in some cases turned by means of straps passing over their circumferences. In such cases the minuts protulncrances of the surfaces prevent the sliding of the straps, und a mutual action takes place such as to render the calcuJation exactly analogous to that in the Proposition.

For the calculation of the best forms for the tecth, the render is referred to a I'nper of Mr Airy's, in the Cumb. I\%il. Trans. Vol, II. p. 277.

159. The fourth Mochanical Power is the Pully. There are severnl species of pullieg: we shall mention them in order. The simple pally is a small wheel moveable about its axis: a string passes over part of its circumference. If the axis is fixed the effect of the pully is only to change the direction of the string passing over it: if however the axis bo moreable, then, as will be presently seen, a mechanical advantage may be gained.

It is sometimes assumed as axiomatic that if a perfectly flexible string passes over a smooth surface the tension of the string will be the same throughout; we shall see, however, in the Chapter on Flexible Strings that this result admits of demonstration. In the present Chapter we shall only require a part of the general proposition. We shall suppose the pallies to be circular, and assume that the tensions of the two portions of any string which are separated by a portion in contact with a pully are equal. And this may be shewn to be necessarily true if we merely admit that the string is a tangent to the circle at the point where it ceases to be in contact with the pully. For since the pully is smooth the directions of all the forces which it exerts on the string must pass throngh the centre of the prolly: hence if wo take the moments with respect to this point of the forces which act on the string we see that the string cannot be in equilibrium unless the tensions of the two portions are equal. 
159. To find the ratio of the pover and weight in the single moreable Pully.

I. Suppose the parts of the string divided by the pully are parallel.

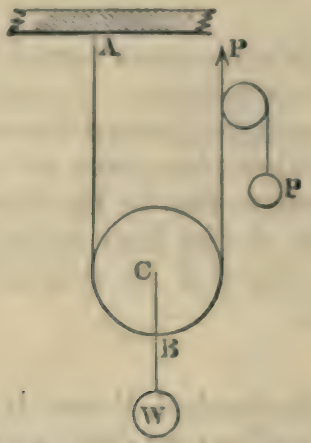

Let the string $A B P$ have one extremity fixed at $A$, and after passing under the pully at $B$ suppose it held by the hand exerting a force $P$, or it may be passed over a fixed pully. The weight $W$ is suspended by a string from the centre $C$ of the pully.

Now the tension of the string $A B P$ is the same throughout. Hence the pully is acted on by three parallel forces, $P, P$, and $W$; hence

$$
2 P-W=0 ; \text { therefore } \frac{W}{P}=2 .
$$

II. Suppose the portions of the string are not parallel.

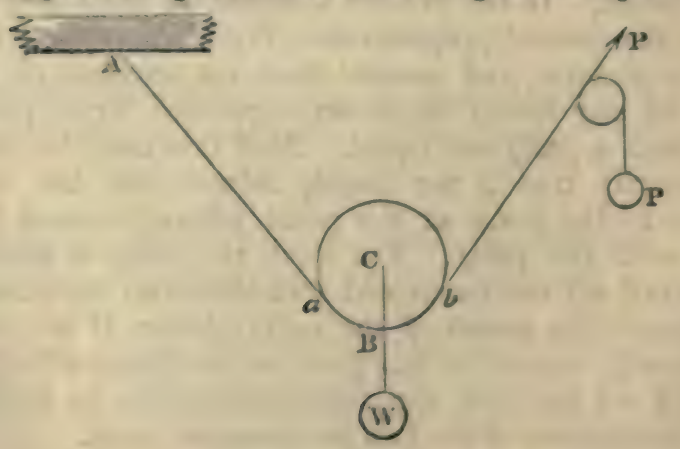


Tet $a$ and $a^{\circ}$ be the angles which $A a$ and $P b$ make with the vertical.

Now the pally is held in equilitrium by $W$ in $C W, P$ in $\alpha A$, and $P$ in $C P$. Hewce, resolving the forces horizontally and vertically,

$$
P \sin a-P \sin a^{\prime}=0
$$

$$
P \cos a+P \cos a^{\prime}-W=0
$$

the equation of moments round $C$ is an identical equation.

By (1),

therefore, by (2),

$$
\sin a=\sin a^{\prime} \text { and } a=a^{\prime} \text {; }
$$

$$
\frac{W}{P}=2 \cos \alpha,
$$

which is the relation required.

160. To find tho ratio of the poucer and weright in a system of pullies, in which each pully hangs from a fircel point by a soparute string, one end being fusteneal in the pully above it and the ether enel on a fixed beam, and all the strings being parallet.

Let $n$ be the number of moveable pullies.

I. Let us neglect the weight of the pullies themselves. Then

$$
\begin{aligned}
& \text { tension of } b_{2} W=W ; \\
& \text { tension of } a_{2} b_{2} b_{2}=\frac{1}{2} W ; \\
& \text { tension of } a_{2} b_{2} b_{3}=\frac{1}{2^{2}} W ; \\
& \text { tension of } a_{3} b_{3} c=\frac{1}{2^{3}} W ;
\end{aligned}
$$

and 80 on; and the tension of the string passing under the $n^{\text {th }}$ pully $=\frac{1}{2^{*}}, W$, and this is equal to $P$; therefore

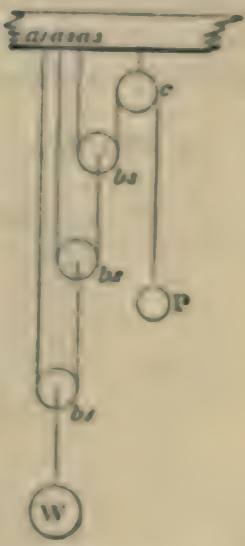

$$
\frac{W}{P}=2 *
$$


II. Let us suppose the weights of the pullies to be considered; and let $\omega_{1}, \omega_{2}, \omega_{3}, \ldots \omega_{n}$ be these weights.

Then if $p_{1}, p_{2}, p_{3}, \ldots p_{n}$ be the weights which they would sustain at $P$, and $P_{1}$ the weight which $W$ would sustain at $P$, we have

$$
p_{1}=\frac{\omega_{1}}{2^{n}}, \quad p_{3}=\frac{\omega_{2}}{2^{n-1}}, \ldots . . p_{n}=\frac{\omega_{n}}{2}, \quad P_{1}=\begin{aligned}
& W \\
& 2^{n}
\end{aligned} ;
$$

therefore

$$
P=p_{1}+p_{2}+\ldots \ldots+p_{n}+P_{1},
$$

or $P=\frac{1}{2^{n}}\left\{W+\omega_{1}+2 \omega_{2}+2^{2} \omega_{3}+\ldots \ldots+2^{n-1} \omega_{n}\right\}$.

If $\omega_{1}=\omega_{2}=\omega_{3}=\ldots \ldots=\omega_{n}$,

$$
P=\frac{1}{2^{n}}\left\{W+\left(2^{n}-1\right) \omega_{1}\right\} \text {, that is } P-\omega_{1}=\frac{1}{2^{n}}\left(W-\omega_{1}\right) \text {. }
$$

161. To find the ratio of the power and weight when the system is the same as in the last Proposition, but the strings are not parallel.

We shall neglect the weights of the blocks. The pullies will evidently so adjust themselves that the string at the centre of any pully will bisect the angle between the strings touching its circumference.

Let $2 x_{1}, 2 x_{2}, 2 x_{3}, \ldots 2 x_{n}$ be the angles included between

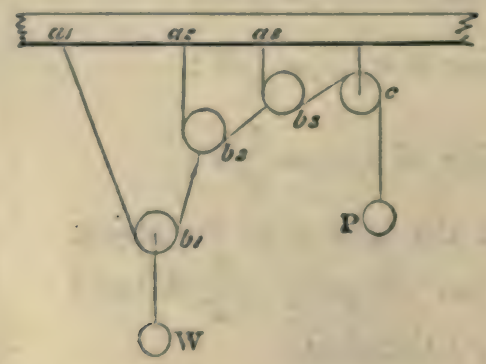

the strings touching the first, second, third, $\ldots \ldots n^{\text {th }}$ pullies respectively. 
5. Then, by Art. 159,

$$
\begin{aligned}
& \text { tensiun of } a_{1} b_{1} b_{3}=\frac{W}{2 \cos \alpha_{1}} \text {; } \\
& \text { tunvion of } a M_{3}=\frac{W}{2 \cos x_{3} \cos \xi_{3}} \text {; } \\
& \text { temsion of } a_{2} f_{2}=\frac{W}{z \cos a_{1} \cos \theta_{2} \cos \sigma_{2}} \text {; }
\end{aligned}
$$

tension of the last string $=\frac{W}{2^{*} \operatorname{coss} \alpha_{2} \cos \alpha_{2} \cos \alpha_{2} \ldots \cos \alpha_{a}}$,

and this is equal to $P$; therefore

$$
\frac{W^{\circ}}{P^{\prime}}=2^{\circ} \cos \alpha_{1} \cos \alpha_{2} \cos \sigma_{0} \ldots \cos \alpha_{0} \text {. }
$$

162. To find the relution of the porer and wexigh in a oysen of pulices veliere the some string passes round all the pullies.

This system consists of two blocks, each containing a number of the pullies with their axes coincident. The wcight is suspended from the lower block, which is movealile, and the power acts at the loose extremity of the string. which passes round the respective pullies of the upper and lower block alternately.

Since the same string passes round all the pullies, its tension will be everywhere the same, and equal to the power P. Let $n$ be the number of portions of string at the lower block; then n. P'will be the sum of their tensions; therefore

$$
W=n \cdot P \text {. }
$$

If we take into account the weight of the lower block, and call it $B$, then

$$
W+B=n \cdot P \text {. }
$$

If the strings at the lower lock are not rertioal, we must take the sum of the parts resolred rertically, and equate is to II: But in general this deviation from the vertical is so Blight that it is ueglected. 
163. As the weight is rising or falling, it will be otserved that in general the pullies move with different angular motions. The degree of angular motion of each pully depends upon the magnitude of its radius. Mr James White took advantage of this, to choose the radii of the pullies in such a manner as to give those in the same block the same angular motion, and so to prevent the wear and resistance caused by the friction of the pullies against each other. 'This being the case, the pullies in each block might be fastened together, or, instead of this, cut out of one mass.

It will be seen without much difficulty, that if the weight $W$ be raised through a space $a$, each of the portions of string between the two blocks will be shortened by the length $a$; and therefore, that the portions of string which move over the pullies in the two blocks, taken alternately, will have their lengths equal to $a, 2 a, 3 a, 4 \pi \ldots$ Suppose the end of the string fastened to the lower block; then if the radii of the pullies of the upper block be proportional to the odd numbers $1,3,5, \ldots \ldots$ these pullies will move with the same angular velocity, and might be made all in one piece, as mentioned above. And it the radii of the lower pullies be proportional to the even integers $2,4,6, \ldots$ these also will move with a common angular velocity, and might therefore be cut out of one piece.

164. To find the ratio of the power to the weight when all the strings are attached to the weight.

If we neglect the weights of the pullies, the tension of the string $b_{1} a_{1}=P$; the tension of $a_{2} l_{3}=2 P$; and so on: if there be $n$ pullies, then the sum of the tensions of the strings attached to the weight

$$
\begin{gathered}
=P+2 P+2^{2} P+\ldots+2^{n-1} P=\left(2^{n}-1\right) P ; \\
\text { therefore } \frac{W}{P}=2^{n}-1 .
\end{gathered}
$$

If we suppose the weights of the pullies are $\omega_{1}, \omega_{n}, \omega_{3} \ldots$ recknning from the lowest, and $\omega, \omega, \omega, \ldots$ the portions of $W$ which they respectively support, since they evidently assist $P$, and $W^{\prime \prime}$ the portion of $W$ supported by $P$; then

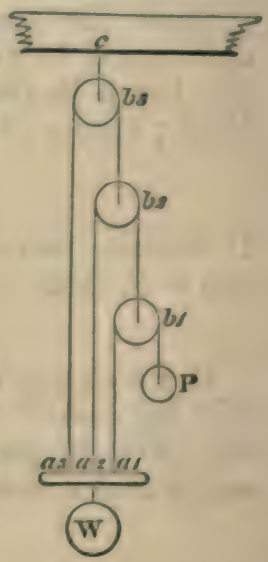




$$
\begin{aligned}
& W^{\prime \prime}=\left(2^{n}-1\right) P \text {, } \\
& \omega^{\prime}=\left(2^{*-1}-1\right) \omega_{1}, \\
& \omega^{\prime \prime}=\left(2^{* 1}-1\right) \omega_{3} \text {, } \\
& \sigma^{-11}=(2-1) \omega_{-1} ;
\end{aligned}
$$

therefore $W=W^{n}+\omega^{\prime}+\ldots \ldots=\left(2^{n}-1\right) P+\left(2^{n-1}-1\right) \omega_{1}$

$$
+\left(2^{m-1}-1\right) \omega_{2}+\ldots \ldots+(2-1) \omega_{b-1} \text {. }
$$

If $\omega_{1}=\omega_{1}=\omega_{1}=\ldots . . .$.

$$
\begin{aligned}
H^{\circ} & =\left(2^{0}-1\right) P+\left(2^{n-1}+2^{n-1}+\ldots . .+2-(n-1)\right\} \omega_{1}, \\
& =\left(2^{n}-1\right) P+\left(2^{*}-n-1\right) \omega_{1} .
\end{aligned}
$$

16.5. The fifth Mechanical Power is the Inclined Plane.

By an inclined plane we mean a plane inclined to the horizon. A weight $W$ may be supported on an iuclined plane by a power $P$ less than $W$.

16R. To find the ratio of the poter and sccight in the inclineal plane.

Let $A B$ be the inclined plane; a the angle which it makes with the horizon. Let the power $P$ act on the weight in the direction $C P$, making an angle $\epsilon$ with the plane. Now the weight at $C$ is held at rest by $P$ in $C P, W$ in the vertical $O W$, and a pressure $R$ in $C R$, at right angles to the plane.

Hence, by Art. 27, if we reaolve these forces perpendicular and parallel to the plane, we have

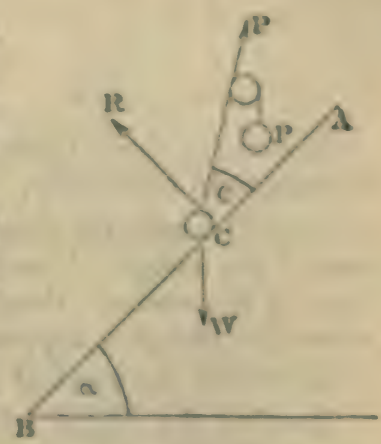

$$
\begin{aligned}
R+P \sin \varepsilon-W \cos \alpha & =0 \ldots \ldots \ldots \ldots \ldots(1), \\
P \cos \epsilon-W \sin \alpha & =0 \ldots \ldots \ldots \ldots(2) .
\end{aligned}
$$

The sccond equation gives the required relation $\frac{P}{11}=\frac{\sin \alpha}{C+5 e}$; and the first eguation gives the macnimde of the pressure $R$.

If $\dot{P}$ act horizuntally, $\epsilon=-a$, and $P=W$ tau $a$. 
If $P$ act parallel to the plane, $\epsilon=0$, and $P=W \sin \alpha$.

If $P$ act vertically, $\epsilon=\frac{1}{2} \pi-\alpha$, and $P=W$.

167. The sixth Mechanical Power is the Wedge. This is a triangular prism, and is used to separate obstacles liy introducing its edge between them and then thrusting the wedge forward. This is effected by the blow of a hammer or otber such means, which produces a violent pressure for a sliort time, sufficient to overcome the greatest forces.

168. An isosceles wedge is kept in equilibrium by pressures on its three fuces; to find the relation between them.
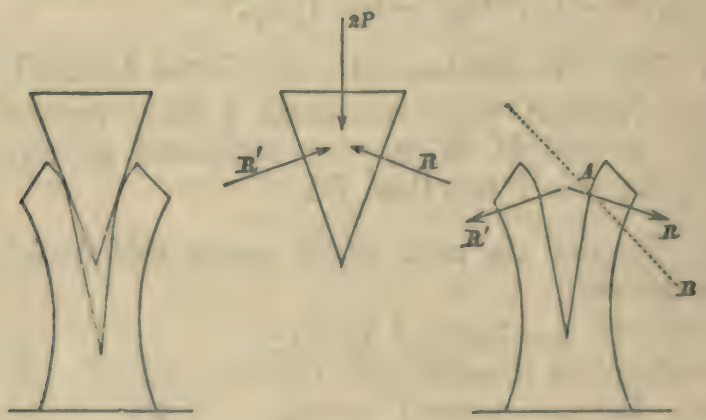

The above three figures represent the wedge and obstacles together and separately.

Let $2 P$ denote the force acting perpendicularly to the thick end of the wedge; $R$ and $R^{\prime}$ the forces which act on the other faces of the wedge: these forces are perpendicular to the faces since the wedge is supposed smooth.

Let $2 a$ be the vertical angle of the wedge.

Resolve the forces which act on the wedge in directions perpendicular and parallel to the thick end; then for the equilibrium of the wedge we have

$$
2 P=\left(R+R^{\prime}\right) \sin \alpha_{1}
$$

$$
R \cos \alpha=R^{\prime} \cos \alpha \text {; }
$$

therefore

$$
\begin{aligned}
& R=R^{\prime}, \\
& P=R \sin \alpha .
\end{aligned}
$$


We do not write down the equations of equilibrium of the obatacle, because we do not know the forces exerted on it at different points of its hase by the ground on which it regts,

It is usual to resulve the force $R$ which acts on the wedge and obstacle into two components; one along the straight line in which $A$, the point of the obstarle in contact with the wedge, would lergin to move if the wedge were pushed further into the obstacle, and the other at right angles to this direction. Iet $A B$ be the first direction, making an angle $i$ with the dircetion of $R$; then the resolval part of $R$ in this direction is $R$ cos $i$, which wo will call $S$;

therefore

$$
\frac{P}{S}=\frac{\sin \alpha}{\cos i} .
$$

As however nothing is known about the value of the angle $i$, the resule is of no practical value.

169. The last Mechanical Power is the Serew. This machine in its simple construction consists of a cylinder $A B$ with a uniform projocting thread abod... traced round its surface, and making a constant angle with straight lines parallel to the axis of the cylinder. This cylinder fits into a block $D$ piered with an equal cylindrical aperture, on the inner surface of which is ent a groose the exact counterpart of the projecting thread abod......

It is easily seen from this de-
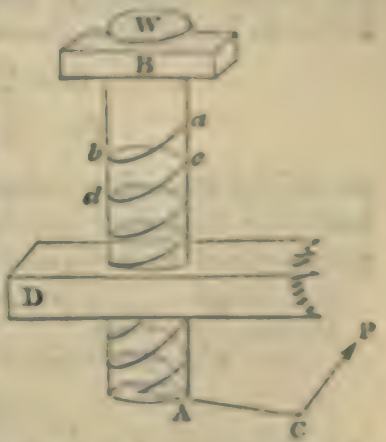
scription, that when the cylinder is introduced into the block, the only manner in which it can move is backwarls or forwards by revolving about its axis, the thread sliding in the groore. Suppose $W$ to be the weight acting on the cylinder, including the weight of the cylinder itself, and $I$ ' to be the power acting at the end of an arm $A C$ at right angles to the axis of the cylinder; the block $D$ is supposed to be firmly fixed, and the axis of the cylinder to be vertical.

170. To find the ratio of the pouser and uccight in the Screte tehen they are in equilibrium.

T. 8. 
Let the distance of $C$ from the axis of the cylinder $=a$; and the radius of the cylinder $=b$.

Now the forces which hold the cylinder in equilibrium are $W, P$, and the reactions of the pressures of the various portions of the thread on the corresponding portions of the lower surface of the groove in which the thread rests; these reactions are indeterminate in their number but they all act in directions at right angles to the surface of the groove, and therefore their directions make a constant angle with the axis of the cylinder. Let $\frac{\pi}{2}-\alpha$ be the angle which the thread of the screw makes with the axis of the cylinder, then $\alpha$ is the angle which the direction of each reaction makes with the axis of the cylinder. If, then, $R$ be one of these reactions, $R \cos \alpha, R \sin \alpha$ are the resolved parts vertically and horizontally; the horizontal portions of the reactions act each at right angles to a radius of the cylinder. Hence, resolving the forces vertically, and also taking the moments of the forces in horizontal planes, we have

$$
\begin{aligned}
& W-\Sigma . R \cos \alpha=0 \text {.................. (1), } \\
& P a-\Sigma, R b \sin \alpha=0 \ldots \ldots \ldots \ldots \ldots \ldots . .(2):
\end{aligned}
$$

we might write down the other four equations of equilibrium, but they introduce unknown quantities with which we are unconcerned in our question.

Hence $\frac{W}{\psi}=\frac{a \cos \alpha \Sigma \cdot R}{b \sin \alpha \Sigma \cdot R}$, because $b$ and $\alpha$ are constant,

$$
=\frac{a \cos \alpha}{b \sin \alpha}=\frac{2 \pi a}{2 \pi b \tan \alpha}
$$

$=\frac{\text { circumference of circle of which the radius is } a}{\text { vertical dist. between two successive winds of the thread }}$.

The Screw is used to gain mechanical power in many ways. In excavating the Thames Tunnel, the heavy iron frame-work which supported the workmen was gradually advanced by means of large screws. 


\section{MUSCLI.I.ANEOUS EXAMPLES.}

1. If one arm of a common balance bo longer than the other, shew that the real weight of any body is the geonnetrical mean between its apparent weights as weighed first in ove scale and then in the other.

2. The arms of a false balance are unequal, and one of the seales is loaited; a body whose true weight is Plbes appears to weigh W/lhs. when placed in one scale, and $W^{\prime}$ lbs. when placed in the other scale; find the ratio of the arms and the weight with which the scale is loadod.

$$
\text { Iiesults. } \frac{W^{\prime \prime}-P}{P^{\prime}-W} ; \frac{W^{\prime} W^{\prime}-P^{\prime}}{P-W} \text {. }
$$

3. A triangular lamina $A B C$, whose weight is $W$, is suspended by a string fastened at $C$; find the weight which must be attached at $B$ that the vertical through $C$ may bisect the angle $\triangle C B$.

$$
\text { Resule. } \frac{W}{3} \frac{b-a}{a} \text {. }
$$

4. Two equal weights are suspended by a string passing freely over three tacks, which form an isosceles triangle whose base is horizontal; find the vertical angle when the pressure on each tack is equal to one of the weights. Resuls. $120^{\circ}$.

5. A uniform heavy rod, at a given point of which a given weight is attached, is sustained at one end; determine its length when the force which applied at the other eud wiid keep it horizontal is least.

6. $\triangle B G C, D E F$ are two horizontal levers without weight; $B, F$ their fulcrums: the end $D$ of one lever reots on the end $U$ of the other; $I I K$ is a rod without weight suspended by two equal parallel strings from the points $E, G$. Prove that a weight $P$ at $\boldsymbol{A}$ will balance a weight $W$ placed anywhere ou the rod $I I K$, provided

$$
\frac{E F}{D F}=\frac{B G}{B C} \text { and } \frac{P}{W}=\frac{B G}{A B} \text {. }
$$


7. If the axis about which a wheel and axle turns coincide with that of the axle, but not with the axis of the wheel, find the greatest and least ratios of the power and weight necessary for equilibrium, neglecting the weight of the machine.

S. In the system of pullies where each string is attached to the weight, let one of the strings be nailed to the block through which it passes, then shew that the power may be increased up to a certain limit without producing motion. If there be three pullies, and the action of the middle one be checked in the manner deseribed, find the tension of each string for given values of $P$ and $W$.

9. A weight 20 is supported on an inclined plane by two forces, each equal to $\frac{w}{n}$, one of which acts parallel to the G base. Shew that equilibrium may be possible when the in$M$ clination of the plane is not greater than $2 \tan ^{-1}\left(\frac{1}{n}\right), n$ being a positive integer.

10. $\Lambda$ weight is suspended from the two ends of a straight lever without weight whose length is 5 feet, by strings whose lengths are 3 and 4 feet. Find the position of the fulcrum that the lever may rest in a horizontal position.

Result. At a distance $3 f$ feet from that end of the lever to which the longer string is fastened.

11. A uniform steelyard $A B$, having a constant weight $P$, and a scale-pan of weight $k P$, suspended at $B$ and $A$ respectively, is used as a balance by moving the rod backwards and forwards upon the fulcrum $C$, on which the whole rests. Shew that the beam must be graduated by the formula

$$
\Delta C=\frac{1+\frac{1}{2} k^{\prime}}{n+k+k^{\prime}+1} \cdot A B
$$

the weight of the rod being $k^{\prime} P$, and $n$ being each of the natural numbers $1,2,3, \ldots$ taken in succession. 
12. $A B$ is a rod withont weight enpable of turning freely about its extremity $A$, which is fixed; $C D$ is another rod equal to $2.1 B$, and attached at its middle point to the extremity $B$ of the former, so as to turn freely about this print; a given fores acts at $O$ in the direction CA, find the furce which must he applied at $D$ in order to produce equilitrium.

13. A lever without weight in the form of the are of a cirele, having twe weights $P$ and $Q$ suspended from its estremities, rests with its couvexity downwards on a horizontal plane; determine the position of equilibrium.

liesult. Let a be the angle which the are subtends at the centre of the circle, $\theta$ the inclination to the rertical of the rndius at the extremity of which $P$ is suspended; then

$$
\tan \theta=\frac{Q \sin a}{P+Q \cos a} \text {. }
$$

14. The sides of a rhombus $\triangle B C D$ are hinged topether at the angles; at $A$ and $C$ are two pulling forees $(P, F)$ acting in the diagonal $A C$; and at $B$ and $D$ there are two other pulling forces $(Q, Q)$ acting in $B D$; shew that

$$
\cos B A D=\frac{P^{2}-Q^{\prime}}{P^{n}+Q^{\circ}}
$$

15. $A B, B Q$ are two equal and uniform beams connected by a hinge at $B$; there is a fixed hinge at $A$; a string fastened at $C$ passes over a pully at $D$ and is attached to a weight $P^{p} ; A D$ is horizontal and equal to twice the length of either beam; shew that if $P$ be such as to keep $B C$ horizontal $P=W \cdot \sqrt{ }$, and $\tan \theta=2 \tan \phi=2 \sqrt{2}$, where $\theta$ and $\phi$ are the angles which $A B, C D$ make with the horizon, and $2 W$ the weight of each beam.

16. A string $\triangle B C D E P$ is attached to the centre $A$ of a pully whose radius is $r$; it then passes over a fixed point $B$ and under the pully which it touches at the points $O$ and $D$ : it afterwards passes over a fixed point $E$ and has a weight $P$ attached to its extremity; $B E$ is horizontal and $=\$ r$, and $D E$ is rertical; shew that if the system be in equilibrium the weight of the pully is $s P$, and find the distance $A B$.

$$
\text { Resule. } A B=\frac{8 r}{3 \sqrt{7}} \text {. }
$$


17. Three uniform rods rigidly connected in the form of a triangle rest on a smonth sphere of radius $r$; shew that the tangent of the inclination of the plane of the triangle to the horizon is $\frac{a}{\sqrt{\left(r^{3}-\rho^{2}\right)}}$, where $a$ is the distance of the centres of the circles inscribed in the triangle itself and in the triangle formed by joining the middle points of the rods, and $\rho$ is the radius of the circle inscribed in the triangle.

18. If a steelyard be constructed with a given rod whose weight is inconsiderable compared with that of the sliding weight, the sensibility varies inversely as the sum of the sliding weight and the greatest weight which can be weighed.

19. A heavy equilateral triangle hung up on a smooth peg by a string, the ends of which are attached to two of its angular points, rests with one of its sides vertical; shew that the length of the string is double the height of the triangle.

20. Three equal heavy spheres lying in contact on a horizontal plane are held together by a string which passes round them. $\Lambda$ cube, whose weight is $W$, is placed with one of its diagonals vertical so that its lower faces touch the spheres; shew that the tension of the string is not less than $\frac{W \sqrt{ } 2}{3 \sqrt{3}}$.

21. A roof of given span is to be constructed of two beams, which are to be connected at the vertex by a single pin, and the weight of the roof would increase in proportion to the length of the beams; what will be the angle of inclination to the horizon, when the whole pressure on the wall is the least possible?

Shew that the direction of the line of pressure will then make the same angle with the vertical line which the beam makes with the horizontal line.

22. $\Lambda \mathrm{n}$ endless string supports a system of equal heary pullies, the highest of which is fixed, the string passing round every pully and crossing itself between each. If $\alpha, \beta, \gamma, \& c$. be the inclinations to the vertical of the successive portions of string, prove that $\cos \alpha, \cos \beta, \cos \gamma, \&$. are in arithmetical progression. 
23. Three equal henvy cylinders, each of which tovelies the other two, are bound together by a atring and laid on a horizontal table so that their axes are horizontal; the tenaion of the string being given, find the pressures between the eylinders.

Iiesults. The horizontal pressure $=T-\frac{W}{2 \sqrt{3}}$, the other $=T+\frac{W}{\sqrt{3}} ;$ where $T$ is the tension of the string, and $W$ the weright of each cylinder.

24. A string of equal spherieal bends is placed upon a monoth eone having its nxis vertieal, the beads being just in enntact with eacl! other, so that there is no mutual pressure between them. Find the tension of the string: and deduce the limiting value when the number of beads is indefinitely great.

25. A smooth cylinder is supported on an inclined plane with its axis herizuntal, by means of a string which, passing over the upper surface of the cylinder, has one end uttached to a fixed point and the other to a weight $W$ which hangs freely; if a be the inclination of the plane to the horizon, and $\theta$ the inclination to the vertical of that part of the string which is fnstened to the fixed point, the weight of the eylinder is

$$
2 I 1 \frac{\sin \frac{1}{2} \theta \cos \left(\alpha+\frac{1}{2} \theta\right)}{\sin \alpha} \text {. }
$$

26. An inextensible string binds tiglitly together two smooth cylinders whose radii are giren; find the ratio of the pressure between the cylinders to the tension by which it is produced.

Resule. $\frac{4 \sqrt{ }\left(r_{1} r_{3}\right)}{r_{1}+r_{3}}$; where $r_{1}$ and $r_{2}$ are the given radii.

27. A ring whose weight is $P$ is movesble along a smooth mol inclined to the horizon at an angle $a$; another ring of weight $P$ is noveable along a rod in the same vertical plane as the former and inclined at an angle $\alpha$ to the horizon; a string which connects these rings passes through a third ring of weight $2 \mathrm{~W}$; shew that the syatem cannot be in equilibrium unless

$$
P \tan a-P^{\prime} \tan a^{\prime}+W^{\prime}\left(\tan \alpha-\tan a^{\prime}\right)=0 .
$$


28. A ball of given weight and radius is hung by a string of given length from a fixed point, to which is also attached another given weight by a string 80 long that the weight hangs below the ball; find the angle which the string to which the ball is attached makes with the vertical.

Result. Let $Q$ be the weight of the ball, $P$ the weight which hangs below the ball, $a$ the radius of the ball, $l$ the length of the string; then the inclination of the string to the vertical is $\sin ^{-1}\left(\frac{P}{P+Q} \cdot \frac{a}{a+l}\right)$.

29. A right cone whose axis is $a$ and vertical angle is $2 \sin ^{-1} \sqrt{\left(\frac{3}{7}\right)}$ is placed with its base in contact with a smooth vertical wall, and its curved surface on a smooth horizontal rod parallel to the wall; shew that it will remain at rest if the distance of the rod from the wall be not greater than $a$ nor less than $\frac{a}{7}$.

30. $\Lambda$ paraboloid is placed with its vertex downwards and axis vertical between two planes each inclined to the horizon at an angle of $45^{\circ}$; find the greatest ratio which the height of the paraboloid may have to its latus rectum, so that, if it be divided by a plane through its axis and the line of intersection of the inclined planes, the two parts may remain in equilibrium: also find the least ratio.

Result. Let $h$ be the height and $4 a$ the latus rectum; then the greatest and least ratios are determined respectively by

$$
3 a=\frac{32}{15 \pi} \sqrt{ }(a h), \quad h+\frac{32}{15 \pi} \sqrt{ }(a h)=3 a .
$$

31. Three bars of given length are maintained in a horizontal position, and tied together at their extremities so as to form a horizontal triangle; and a smooth sphere of given weight and size rests upon them. Find the pressure of the sphere on each bar. 
32. One end of a string is fastened to a print in a smocts vertical wall, the other to a point in the circumference of the hase of a eglinder; the eylinder is in equilibrium, having a print of its upper end in contact with the wall: fint the distance of this juint below the point in the wall to which the string is fastened.

liesult. Suppose $x$ the required distance, $l$ the length of the string, $f$ the height of the eglimler, $b$ the diameter of its base; then

$$
3 x^{2}=l^{2}-h^{2}-b^{2}
$$

33. The ends of a string are fastened to two fixed proints, and from knots at given points in the string given weiglits are hung: shew that the horizuntal component of the tension is the same for all the portions into which the string is divided by the knots. Shew also that if the weights are all equal the tangents of the angles which the successive portions of the string make with the horizon are in Arithmetival Progression. (Fuch a system is called a Finicular Polggem.)

34. Two uniform beams locsely jointed at one extremity are placed upon the smooth arc of a paratola, whose axis is vertical and vertex upwards. If $l$ be the semi-latus reetum of the parabola, and $a, b$, the lengths of the beams, shew that they will rest in equilibrium at right angles to each other, if

$$
l(a+b)\left(a^{6}+b\right)^{1}=a^{4} b^{4} ;
$$

and find the position of equilibrium.

35. A quadrilateral is formed by four rigid rods jointed at the ends: shew that two of its sides must we parallel in orter that it may preserve its form when the millde points of either pair of epposite sides are joined together by a string in a state of tension.

36. Four rods, jointed at their extremities, form a quadrilateral, which may be inseribed in a circle; if they be kept in equilibrium by two strings joining the opposite angular points, shew that the tension of each string is inversely proportional to its length.

37. Four equal and uniform heary rods being jointed by 
hinges so as to form a square, two opposite angles are connected by a string; this frame-work stands on a fixed point, the string being horizontal; find the tension of the string.

Result. Twice the weight of a rod.

38. Four equal and uniform heary rods are connected by hinges; the system is suspended by a string attached to one hinge, and the lowest hinge is in contact with a horizontal plane; find the tension of the string and the pressure on the plane.

Result. Each is twice the weight of a rod.

39. A regular hexagon, composed of six equal heavy rods moveable about their angular points, is suspended from one angle which is connected by threads with each of the opposite angles. Shew that the tensions of the threads are as $\sqrt{ } 3: 2$. Find also the strain along each rod.

40. A regular hexagon is composed of six equal heavy rods moveable about their angular points; one rod is fixed in a horizontal position, and the ends of this rod are connected by vertical strings with the ends of the lowest rod; find the tension of each string.

Result. $\frac{3}{2} \mathrm{~W}$; where $W$ is the weight of a rod.

41. Suppose that in the preceding Example each end of the fixed rod is connected with the more remote end of the lowest rod, so that the strings instead of being parallel are inclined at an angle of $60^{\circ}$; find the tension of each string.

Result. $W \sqrt{ } 3$.

42. A regular hexagon is composed of six equal heavy rods moveable about their angular points, and two opposite angles are connected by a horizontal string; one rod is placed on a horizontal plane, and a weight is placed at the middle point of the highest rod; find the tension of the string.

Result. Let $W$ be the weight of each rod, and $W^{\prime}$ the weight placed on the highest rod; then the tension is

$$
\frac{3 W+W^{\prime}}{\sqrt{ } 3} \text {. }
$$




\section{$203)$}

\section{CHAPTER $\mathrm{x}$.}

\section{FRICTIO\&.}

171. Is the investigations of the preceding Chapter, we have supposed that the surfaces of the bodies in cintact are perfectly smooth. By a smoolh surface is meant a surface which opposes no resistance whatever to the motion of a body upon it. A surface which does oppose a resistance to the motion of a body upon it is said to be rough. In practice it is found that all bodies are more or less rough.

The friction of a body on a surface is measured by the least force which will put the body in motion along the surface.

172. Coulomb made a series of experiments upon the friction of bodies against each other and dednced the following laws. Memoires...... des Sueans Eirangers, Tom. $\mathbf{x}$.

(1) The friction varies as the normal pressure schen the materials of the surfaces in contact remain the same. When the pressures are very great indeed, it is found that the friction is somewhat less than this law would give.

(2) The frivion is independent of the crtont of the surfaces in contenct so long an the normal pressure remains the same. When the surfaces in contact are extremely small, as for inmtance a cylinder reating on a surface, this law gives the friction much too great.

These two laws are true when the body is on the point of moving and also when it is actually in motion; but in the crae of motion the magnitnde of the friction is not alwags the same as when the body is in a state bordering on motion: when there is a difference the friction is greater in the state bordering on motion than in actual motion.

(3) The friction is independent of the velucity wiven the larty is in motion. 
It follows from these laws that if $P$ be the normal pressure between two surfaces, then the friction is $\mu P$, where $\mu$ is a constant quantity for the same materials and is called the coefficient of friction.

The following results, selected from a table given by Professor liankine, will afford an idea of the amount of friction as determined by experiment; these results apply to the friction of motion.

For iron on stone $\mu$ varies between 3 and 7 .

For timber on timber ................. $\cdot 2$ and $\cdot 5$.

For timber on metals ................... $\cdot 2$ and 6 .

For metals on metals .................. 15 and $\cdot 25$.

For full particulars on this subject we refer the reader to Coulomb's papers, and to the Memoirs published in the Mémoires de l'Institut, by M. MIorin; see also Rankine's Mfanual of Applied Mechanics, and Moseley's Mechanical Principles of Engineering and Architecture.

173. Angle of Friction. Suppose a body acted on only by its weight to be placed on a horizontal plane and the plane to be turned round a horizontal line until the body begins to slide. Let $W$ be the weight of the body and $a$ the angle the plane makes with the horizon. The pressure of the body on the plane will be equal to the resolved part of its weight perpendicular to the plane, that is to $W \cos \alpha$. The friction is equal to the resolved part of the weight parallel to the plane, that is to $W \sin \alpha$. If $\mu$ be the coefficient of friction, we have

$$
W \sin \alpha=\mu W \cos \alpha ;
$$

therefore

$$
\tan \alpha=\mu \text {. }
$$

This experiment will enable us to determine the value of the coefficient of friction for different substances. The inclination of the plane when the body is just about to slide is called the angle of friction.

174. In Art. 32 we have found the condition of equilibrium of a particle constrained to rest on a smooth curve; we procecd 
to the case of a particle on a rough curve. Suppose the curve a plane curve; let $X, Y$ be the forces which act on the particle parallel to the axen of $x$ and $y$ exelusive of the action of the curve The sum of the resolved parts of $X$ and $Y$ along the tangent to the curve is

$$
X \frac{d x}{d s}+Y \frac{d y}{d s} .
$$

The sum of the resolved parts along the normal is

$$
\pm\left(x \frac{d y}{d s}-Y \frac{d x}{d s}\right) \text {. }
$$

If $\mu$ bo the coefficient of friction the greatest friction capable of being called into action is

$$
\pm \mu\left(x \frac{d y}{d s}-Y \frac{d x}{d s}\right) \text {. }
$$

Ilence, the condition of equilibrium will be that the numerical value of $x \frac{d x}{d_{s}}+Y \frac{d y}{d s}$ must be less than the numerieul value of $\mu\left(x \frac{d y}{d s}-Y \frac{d x}{d s}\right)$, without regard to sign in either case. This may be conveniently expressed thus,

$$
\left(x \frac{d x}{d_{0}}+Y \frac{d y}{d s}\right)^{2} \text { must be less than } \mu^{2}\left(X \frac{d y}{d s}-Y \frac{d x}{d s}\right)^{\prime} \text {. }
$$

We may exhibit this condition in a different form, as will be seen in the following Article.

175. Next let the curve be of dnuble curvature. Let $I^{p}$ denote the resultant force acting on the particle exclusive of the action of the curve; $X, Y, Z$ the components of $P$ parallel to the axes; $l, m, n$ the direction cosines of the tangent to the curve at the point where the particle is placed; $\theta$ the angle between this tangent and the direction of $P$. The resolved part of $P$ along the tangent is $P \cos \theta$, and that at right angles to the tangent is $P \sin \theta$. Hence, if $\mu$ be the coefficient of frietion, we must have for equilibrium

\section{$P \cos \theta<\mu P \sin \theta$;}


206

therefore

therefore

therefore

$$
\cos ^{2} \theta<\mu^{2}\left(1-\cos ^{2} \theta\right) \text {; }
$$

$$
\begin{gathered}
\cos ^{2} \theta<\frac{\mu^{2}}{1+\mu^{2}} ; \\
\left(\frac{X l+Y m+Z n}{P}\right)^{2}<\frac{\mu^{2}}{1+\mu^{2}} .
\end{gathered}
$$

It is easy to shew that this result includes that of the former Article by putting $n=0, m=\frac{d y}{d s}, l=\frac{d x}{d s}$.

176. A particle is constrained to remain on a rough surface: determine the condition of equilibrium.

Let $P$ be the resultant force on the particle exclusive of the action of the surface; $\phi$ the angle between the direction of $P$ and the normal to the surface at the point where the particle is placed; $u=0$ the equation to the surface; $x, y, z$ the coordinates of the particle. The resolved part of $P$ along the normal is $P \cos \phi$, and that at right angles to the normal is $P \sin \phi$. Hence, for equilibrium we must have

$$
P \sin \phi<\mu P \cos \phi \text {; }
$$

therefore

$$
\sin ^{2} \phi<\mu^{2} \cos ^{2} \phi \text {; }
$$

therefore

$$
\cos ^{2} \phi>\frac{1}{1+\mu^{2}}
$$

therefore

$$
\frac{\left(X \frac{d u}{d x}+Y \frac{d u}{d y}+Z \frac{d u}{d z}\right)^{2}}{P^{s}\left\{\left(\frac{d u}{d x}\right)^{2}+\left(\frac{d u}{d y}\right)^{2}+\left(\frac{u u}{d z}\right)^{2}\right\}}>\frac{1}{1+\mu^{2}} .
$$

177. In the following Articles of this Chapter we shall investigate certain equations which hold when the equilibrium of different machines is on the point of being disturbed. The equations in such cases will involve the forces acting on the machine and $\mu$ the coefficient of friction. When we have found one of these limiting equations, we can draw the following inferences :

(1) If in order to satisfy the equation for a given set of forces it is necessary to ascribe to $\mu$ a value greater than its 
extreme value for the subatances in question, whick is known by experiment, equilibrium is impossible.

(2) If the limiting equation can be satisfied by ascribing to $\mu$ values less than its extreme value, equilibrium may be possible. We any may be possible, because the limiting equastion may not be the only equation of equilibrium, and of course for equilibrium is is necessary that all the appropriate equations bo satisfied.

We may illustrate the subject of friction by the solution of the following problem:

$A$ weight $W$ is placed on a rough horizontal plane; a string is atrached to $W$ and passes over a fixed smooth pully, and to the other end of the string a weight $P$ is attached. Betermine the limiting inclinations to the vertical of the string which passes from $W$ to the fixed pully, when there is equilibrium.

Let $\theta$ be the inclination of this string to the vertical. The tension of the string is equal to $P$. The body on the rough horizontal plane is acted on by its own weight, by the resiatance of the plane, which is at right angles to the plane, and by the friction along the plane: denote the resistance by 16 , and the friction by $\mu R$.

Then resolving the forces horizontally and vertically wo have

$$
\begin{gathered}
\mu R=P \sin \theta, \\
R+P \cos \theta=W .
\end{gathered}
$$

Ilence, climinating $R$, we have

$$
\begin{gathered}
\mu(W-P \cos \theta)=P \sin \theta ; \\
\mu=\frac{P \sin \theta}{W-P^{\prime} \cos \theta} \\
=\frac{\sin \theta}{k-\cos \theta^{\prime}}
\end{gathered}
$$

therefore

where $k$ is put for $\frac{W}{P}$.

We may, without any real loss of generality, suppose that $\theta$ is a positive angle not exceeding a right angle. 
It may be shewn that the differential coefliciont of $\frac{\sin \theta}{t-\cos \theta}$ with respect to $\theta$ is $\frac{k \cos \theta-1}{(k-\cos \theta)^{2}}$; this result will be useful as we proceed.

I. Suppose $W$ less than $P$, so that $k$ is less than unity.

Now $\mu$ may have any value from zero up to a certain limit, known by experiment, which we shall denote by $\tan \epsilon$. Thus at the limit

$$
\tan \epsilon=\frac{\sin \theta}{k-\cos \theta},
$$

therefore

$$
\cot \epsilon+\cot \theta=\frac{l}{\sin \theta},
$$

therefore

$$
\sin (\theta+\epsilon)=7 \cdot \sin \epsilon .
$$

Let $\alpha$ be the least angle which has $k \sin \epsilon$ for its sine, so that

$$
\sin (\theta+\epsilon)=\sin \alpha .
$$

And as $\alpha$ is less than $\epsilon$ the only solution admissible here is

$$
\theta+\epsilon=\pi-\alpha
$$

The expression $\frac{\sin \theta}{k-\cos \theta}$ is not positive unless $\theta$ is greater than the value which makes $\cos \theta=k$; and for greater values of $\theta$ the expression decreases as $\theta$ increases, and has its least value with which we are concerned when $\theta=\frac{\pi}{2}$; its value then being $\frac{1}{k}$, that is, $\frac{P}{W}$.

Hence we have the following results:

If the coefficient of friction is less than $\frac{P}{W}$ there is no position of equilibrium, 
If the coefticient of friction is greater than $\frac{P}{W}$ equilibrium will subsist for all values of $\theta$ between $\pi-\alpha-\epsilon$ and $\frac{\pi}{2}$.

II. Suppose $W$ greater than $P, 80$ that $k$ is greater than unity.

In this case $\alpha$ is greater than $e$, and the equation

$$
\sin (\theta+c)=\sin \alpha
$$

has two solutions which may be admissible, namely,

$$
\theta+e=\alpha \text {, and } \theta+e=\pi-a \text {. }
$$

The expression $\frac{\sin \theta}{k-\cos \theta}$ is always positive, and as $\theta$ increases from 0 to $\frac{\pi}{2}$ the expression increases up to a maximum value and then decrenses. The maximum value is when $\cos \theta=\frac{1}{k}$, and is $\frac{1}{\sqrt{\left(k^{3}-1\right)}}$, that is, $\frac{P}{\sqrt{\left(W^{3}-P^{2}\right)}}$.

Hence we shall obtain the following results:

If the coefficient of friction is not less than $\frac{P}{\left.\sqrt{(} W^{2}-P^{2}\right)}$, equilibrium will subsist for all values of $\theta$ between 0 and $\frac{\pi}{2}$.

If the coefficient of friction is less than $\frac{P}{W}$, equilibrium will subsist for all values of $\theta$ between 0 and $a-\epsilon$.

If the coefficient of friction lies between $\frac{P}{W}$ and $\frac{P}{\sqrt{ }\left(W^{2}-P\right)}$, equilibrium will subsist for all values of $\theta$ between 0 and $a-\epsilon$, and between $\pi-\alpha-e$ and $\frac{\pi}{2}$.

III. Suppose $W=P$. In this case there is equilibrium when $\theta=0$, no friction bring then exerted; and besides this we have results which may be dectuced from those in the first ense. Here $\alpha=6$; if the coefficient of friction is less T. 8. 
than unity there is no other position of equilibrium; if the coefficient of friction is greater than unity equilibrium will also subsist for all values of $\theta$ between $\pi-2 \epsilon$ and $\frac{\pi}{2}$.

Or the results for the third case may be deduced from those given in the second case, observing that $\alpha=\epsilon$.

\section{Equilibrium of Machines with Friction.}

\section{Inclined Plane.}

Let $\alpha$ be the inclination of the plane to the horizon. Suppose a force $P_{1}$ acting at an inclination $\theta$ to the plane and the body on the point of moving down the plane. Let $R$ be the normal action of the plane, $\mu R$ the friction which acts up the plane, $W$ the weight of the body. Resolve the forces along and perpendicular to the plane; then, for equilibrium

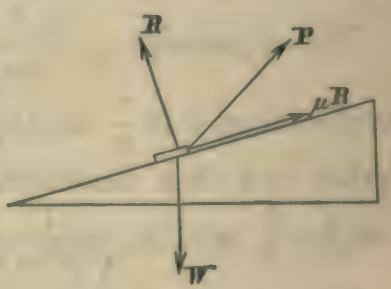
we have

$$
\begin{aligned}
P_{1} \cos \theta+\mu R-W^{\prime} \sin \alpha & =0 \ldots \ldots \ldots \ldots \ldots(1), \\
R+P_{1} \sin \theta-W \cos \alpha & =0 \ldots \ldots \ldots \ldots(2) .
\end{aligned}
$$

Substitute in (1) the value of $R$ from (2); thus

$$
P_{1}=\frac{W \sin \alpha-\mu W \cos \alpha}{\cos \theta-\mu \sin \theta} \text {. }
$$

Next, suppose $P_{8}$ a force acting at an inclination $\theta$ to the plane, such that the body is on the point of moving up the plane. Friction now acts down the plane, and we shall find

$$
P_{2}=\frac{W \sin \alpha+\mu W \cos \alpha}{\cos \theta+\mu \sin \theta} .
$$

This result may be deduced from the former by changing the sign of $\mu$.

There will be equilibrium if the body be acted on by a force $P$, the magnitude of which lies between $P_{1}$ and $P_{2}$. 
Suppose of to be the engle of friction, so that

\section{$\tan e=\mu$,}

then $\quad P_{1}=\frac{W \sin \alpha-\tan \epsilon W \cos \alpha}{\cos \theta-\tan \epsilon \sin \theta}=\frac{W \sin (\alpha-\epsilon)}{\cos (\theta+\epsilon)}$.

Similarly $\quad P=\frac{W \sin (\alpha+e)}{\cos (\theta-\epsilon)}$.

Suppose we reguire to know the least force which will suffice to prevent the body from moving down the plane. The expresuion for $l_{1}$ will be least when $\cos (\theta+6)$ is greatese, that is when $\theta+\epsilon=0$, that is when $\theta=-\epsilon$; and then $P_{8}=W \sin (\alpha-e)$.

Again, suppose we require to know the least force which will suffice to move the tualy up the plates. The expreasion for $P_{\text {s }}$ will he least when cos $(\theta-\epsilon)$ is greatest, that is when $\theta=e ;$ and then $P_{a}=W \sin (a+e)$.

The following, problem will illustrate the snbject of the inclined plane with friction. A weight $W$ is placed on $\Omega$ rough inclined plane, and is attached by a string to a point above the plane: determine the limiting positions of equilibrium.

Iat $a$ be the inclination of the plane to the horizon, $\beta$ the inclination of the string to the plane. $T$ the tension of the string, $I$ the resistance of the plane. Since the body is constrained to remain at a constant distance from the fixed point, it must he situated on the circumference of a certain circle described on the plane; suppose $\theta$ the angular distance of the pisition of the body from the lowest point of the cireump.renve. The forces which sot on the buty at right angles to the plane are $W \cos \alpha, T \sin \beta$, and $R$. Thus

$$
R+T \sin \beta-W \cos a=0
$$

The forces which act on the body in the plane are $W \sin a$, $T$ ous $\beta$, and the friction $\mu R$. Resolve these forces along the radius and tangent at the point of the circumference at which the body rests. 'Thus

$$
\begin{aligned}
T \cos \beta-W \sin \alpha \cos \theta & =0 \ldots \ldots \ldots \ldots \ldots(2), \\
\mu R-W \sin \alpha \sin \theta & =0 \ldots \ldots \ldots \ldots(3) .
\end{aligned}
$$


From (1) and (3) we obtain

$$
\mu T \sin \beta=\mu W \cos \alpha-W \sin \alpha \sin \theta ;
$$

hence by (2)

Therefore

$$
\begin{gathered}
\mu \tan \beta=\frac{\mu \cos \alpha-\sin \alpha \sin \theta}{\sin \alpha \cos \theta} . \\
\mu=\frac{\sin \alpha \sin \theta}{\cos \alpha-\sin \alpha \tan \beta \cos \theta} \\
=\frac{\cot \beta \sin \theta}{\cot \alpha \cot \beta-\cos \theta} .
\end{gathered}
$$

This result may then be developed in the manner already exemplified in Art. 177.

\section{Lever with Friction.}

Suppose a solid body pierced with a cylindrical hole through

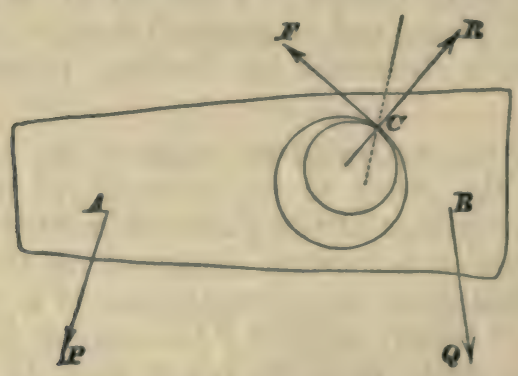

which passes a solid fixed cylindrical axis. Let the outer circle in the figure represent a section of the cylindrical hole made by a plane perpendicular to its axis, and the inner circle the corresponding section of the solid axis. In the plane of this section, we suppose two forces $P$ and $Q$ to act on the solid body at the points $A$ and $B$. Also at the point of contact $C$ there is a normal force $R$ and a tangential force $F$. These four forces keep the body in equilibrium.

Since $R$ and $F$ have a resultant passing through $C$, it follows, by $A$ rt. 58, that the resultant of $P$ and $Q$ must also pass 
through $C$. Iat $\gamma$ be the angle between the directions of $P$ and $Q$, and $S$ the resultant of $P$ and $Q$; then

$$
S^{3}=P^{2}+Q+2 I^{\prime} Q \cos \gamma \text {. }
$$

Let the direction of $S$ be represented by the dotted line making an angle $\theta$ with $\mathbb{R}$. Then since $\vec{F}, \mathbb{R}$, and $S$ are in equilibrium,

$$
\begin{aligned}
& \boldsymbol{R}=\boldsymbol{S} \cos \theta \ldots \ldots \ldots \ldots \ldots \ldots \ldots \ldots(1), \\
& \boldsymbol{F}=\boldsymbol{S} \sin \boldsymbol{\theta} \ldots \ldots \ldots \ldots \ldots \ldots \ldots \text { (2). }
\end{aligned}
$$

For the limiting position of equilibrium $F=\mu R$; therefore

$$
\tan \theta=\mu
$$

We may now find the relation between $P$ and $Q$, by taking moments round the centre of the exterior circle; let $r$ be the radius of this circle; $a$ and $b$ the distances of $A$ and $B$ from its centre; $\alpha$ and $\beta$ the angles which the diroctions of $P$ and $Q$ make with these distances; then

$$
P_{a} \sin a+F r=Q b \sin \beta \text {; }
$$

or by (2) and (3),

$$
P a \sin \alpha+\frac{r \mu}{\left(1+\mu^{2}\right)^{1}}\left(P^{0}+Q+2 P Q \cos \gamma\right)^{\gamma}=Q b \sin \beta \ldots(1) .
$$

If we suppose the friction to act in the opposite direction to that in the figure, we shall obtain

$$
P a \sin \alpha-\frac{r \mu}{\left(1+\mu^{2}\right)^{\frac{1}{3}}}\left(P^{\mu}+Q^{\alpha}+2 P Q \cos \gamma\right)^{\gamma}=Q b \sin \beta \ldots(5) .
$$

Equilibrium will not subsist unless $P, Q, a, b, \alpha, \beta, \gamma$ are $s 0$ adjusted that (4) or (5) ean be satisfied without giving to $\mu$ a value greater than its limit known by experiment.

The following form may be given to the limiting equation. Let $a$ be the length of the perpendicular from the centre of the outer circle on the dotted line. Since $F, I B$, and $S$ are in equilibrium, we have by taking moments

$$
F r=S s \text {; }
$$

thereione

$$
\frac{r \mu}{\left(1+\mu^{2}\right)}=2 .
$$




\section{Wedge soith Friction. (Sec $\Lambda$ rt. 168.)}

Suppose the wedge to be on the point of moving in the direction in which $2 P$ urges it, and assume for simplicity that each face is similarly acted on by the obstacle. The forces which maintain the wedge in equilibrium are $2 P^{P}$ perpendicular to the thick end, $R$ perpendicular to each face, and $\mu R$ along each face towards the thick end. Hence, resolving the forces parallel to the direction of $2 P$,

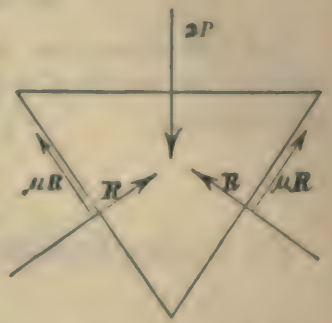

$$
P=R \sin \alpha+\mu R \cos \alpha \ldots \ldots \ldots \ldots \ldots \text { (1). }
$$

Forces equal and opposite to $R$ and $\mu R$ act on the obstacle at each point of contact. If $R^{\prime}$ be the resultant of $R$ and $\mu R$, we have

$$
R^{\prime}=R \sqrt{ }\left(1+\mu^{2}\right)
$$

Let $S$ be the resolved part of $R^{\prime}$ along a direction making an angle $i$ with that of $R$ and $i^{\prime \prime}$ with that of $R^{\prime}$ (sec Art. 168); then

$$
\begin{aligned}
S & =R^{\prime} \cos i^{\prime} \\
& =R \cos i+\mu R \sin i .
\end{aligned}
$$

(1), (2), and (3) will give the ratio of $P$ to $R^{\prime}$ and of $P$ to $S$.

181. Screvo with Friction. (See Arts. 169, 170.)

If the surfaces of the screw are rough it is kept in equilibrium by $W, P$, a system of forces perpendicular to the surface of the groove, and a system of forces arising from friction. Let $R$, denote one of the forces perpendicular to the surface of the groove, $\mu R$, the corresponding friction; then $R$, makes an angle $\alpha$ with the axis of the cylinder on which the screw is raised, and $\mu R$, an angle $\frac{1}{2} \pi-\alpha$ with the axis of the cylinder. Suppose $W$ about to prevail over $P$; then resolving the forces parallel to the axis of the cylinder, and taking moments round it, we have

$$
\begin{aligned}
W-\Sigma R(\cos \alpha+\mu \sin \alpha) & =0, \\
P a-\Sigma R(\sin \alpha-\mu \cos \alpha) b & =0 .
\end{aligned}
$$


'Tiverefore

$$
\begin{aligned}
\frac{P}{W} & =\frac{b}{a}\left(\frac{\sin \alpha-\mu \cos \alpha)}{\cos \alpha+\mu \sin a)}\right. \\
& =\frac{b}{a} \frac{\tan \alpha-\mu}{1+\mu \tan a} \\
& =\frac{b}{a} \tan (\alpha-e) \\
\mu & =\tan \epsilon .
\end{aligned}
$$

if

If we suppose $P$ about to prevail over $W$, we shall find similarly

$$
\frac{P}{W}=\frac{b}{a} \tan (a+c) \text {. }
$$

\section{EXAMPLKS.}

1. A rectanqular prism, whose breadth is 2.83 feet and thickness less than 2 inclies, is laid with its axis horizontal, and with its smaller face on an inclined plane where the cofficient of friction is $\frac{1}{r}$. Shew that if the inclination of the plane is gradually increased, the prism will roll before it will slide.

2. If the ronghness of a plane which is inclined to the burizon at a known angle be such that a boly will just rest supported on it, find the least force requisite to draw the body up.

Results. Ist $a$ be the inclination of the plane, $W$ the weight of the body; then the least force is $W \sin 2 x$, and it acts at an inclination $a$ to the plane.

3. Two rough bodies rest on an inclined plane, and are connected by a string which is parallel to the plane; if the coeflicient of friction be not the same for both, find the greatest inclination of the plane which is consistent with equilibrium.

$$
\text { Resule. } \tan \theta=\frac{\mu_{1} W_{1}+\mu_{2} W_{3}}{H_{2}+H_{3}} \text {. }
$$


4. A rectangular table stands on a rough inclined plane, and has two sides horizontal; if the coeflicient of friction of the lowest feet be $\mu$ and that of the others be $\mu^{\prime}$, find the inclination of the plane when the table is on the point of sliding.

5. Two unequal weights on a rough inclined plane are connected by a string which passes through a fixed pully in the plane; find the greatest inclination of the plane consistent with the equilibrium of the weights.

$$
\text { Result. } \tan \alpha=\frac{\mu\left(W_{1}+W_{2}\right)}{W_{1}-W_{2}} .
$$

6. A heavy uniform rod whose length is $2 a$ is supported by resting on a rough peg, a string of length $l$ being attached to one end of the rod and fastened to a given point in the same horizontal plane with the peg. If when the rod is on the point of sliding the string is perpendicular to it the coefficient of friction is $\frac{l}{a}$.

7. Two weights $P, Q$ of similar material rest on a rough double inclined plane, and are connected by a fine string passing over the common vertex; $Q$ is on the point of motion down the plane, shew that the weight which may be added to $P$ without producing motion is

$$
\frac{P \sin 2 \phi \sin (\alpha+\beta)}{\sin (\beta-\phi) \sin (\alpha-\phi)},
$$

$\alpha, \beta$ being the angles of inclination of the planes and $\tan \phi$ the coefficient of friction.

8. A weight $P$ is attached to a point in the circumference of a rough circular ring whose weight is $W$ : shew that the ring will hang on a horizontal rod in a plane perpendicular to it with any point of the ring in contact with the rod, if the coefficient of friction be not less than

$$
\frac{1}{\sqrt{\left(n^{2}+2 n\right)}} \text {, where } n=\frac{W}{P} \text {. }
$$

9. Two equal heavy rings are moveable on a horizontal rough rod; a string of given length which passes through 
them has both enils altactsed to a giren wright; find the greatest posaible diatance between the ring\%.

10. Three ejual hemiapheres, having their bases downwards, are placed in contact with each other upon a horizontal table; if a smooth spliere of the same substance and equal radius be placed upon them, shew that there will be equilitrum or not, according as the coefficient of friction between the hemisphereas and the table is greater or less than $t \sqrt{2}$.

11. A uniform rod rests wholly within a rough hemispherical bowl in a vertical plane through its centre, prove that the limiting position of equilibrium will be given by the equation

$$
\tan \theta=\frac{\sin 2 c}{2 \cos (\beta+6) \cos (\beta-\epsilon)},
$$

$\theta$ being the inclination of the rod to the horizon, $2 \beta$ the angle it subtends at the centre, and tane the cocfficient of friction.

12. A thin rod rests in a horizontal position between two rough planes equally inclined to the horizon, and whose inclination to each other is $2 x$; if $\mu$ be the coefficient of friction, then the greatest possible inclination of the line of intersection of the planes to the horizon is $\tan ^{-1} \frac{\mu}{\sin \alpha}$.

13. A surface is formed by the revolution of an equilateral hyperbola about one of its asymptotes which is vertical; shew that a particle will rest upon it, supposing it rough, anywhere beyond the intersection of the surface with a certain circular cylinder.

14. A heavy particle under the action of gravity will rest on a rough paraboloid $\frac{x^{2}}{a}+\frac{y^{2}}{\beta}=2 z$, if it be placed on the surface at any point above the curve of intersection of the surface with the cylinder $\frac{x^{3}}{a^{3}}+\frac{y^{3}}{\beta^{3}}=\mu^{2}$; the axis of the parabolvid being vertical, its vertex upwarls, and $\mu$ the coefficient of friction. 
15. A rough elliptic pully of weight $W$ can turn freely about one extremity of its major axis, and two weights, $P$, $Q$, are suspended by a string which passes over the pully; when in equilibrium its plane is vertical, and its axis inclined at $60^{\circ}$ to the horizon, prove that the excentricity of the ellipse is equal to

$$
\frac{\sqrt{ }\{(3 Q+W-P)(Q-W-3 P)\}}{(Q-P) \sqrt{ } 3} .
$$

16. $\Lambda$ heavy hemisphere rests with its convex surface on a rough inclined plane. Find the greatest possible inclination of the plane.

17. One end $A$ of a heavy rod $A B C$ rests against a rough vertical plane; and a point $B$ of the rod is connected with a point in the plane by a string, the length of which is equal to $A B$; determine the position of equilibrium of the rod, and shew how the direction in which the friction acts depends apon the position of $B$.

18. Three equal balls, placed in contact on a horizontal plane, support a fourth ball. Determine the least values of the coefficients of friction of the balls with each other and with the plane, that the equilibrium may be possible.

Results. Let $W$ be the weight of each of the three lower balls, $W^{\prime}$ the weight of the upper, $\phi$ the angle which the straight line joining the centre of the upper ball with the centre of one of the lower balls makes with the vertical; then the coefficient of the friction between the balls is $\tan \frac{\phi}{2}$, and the coefficient of the friction between the balls and the plane is $\frac{W^{\prime}}{W^{\prime}+3 W} \tan \frac{\phi}{2}$. If all four balls are equal we have $\sin \phi=\frac{1}{\sqrt{ } 3}$, so that $\tan \frac{\phi}{2}=\sqrt{ } 3-\sqrt{ } 2$.

19. Determine the curve on the rough surface of an ellipsoid, at every point of which a particle acted on by three equal forces whose directions are parallel to the axes of the ellipsoid, will rest in a limiting position of equilibrium. 
20. BCDE: is a square bond ; a string is fixed to a guint $A$ in a rungh wall and to the corner $B$ of the hotal. Shew that the board will rest with its plane perpendienlar to the wall, and its side $C l l$ resting against it, if $A C$ be not greater than $\mu B C$.

21. A rectangular parallelepiped of given dimmsions is placed with one face in contact with a rough inclined plane; determine the limits of its position in onder that equilibrium may exist.

22. A board, moveable about a horizontal straight line in its own plane, is supported by resting on a rough sphere which lies on a herizontal table; find the greatest inclination at which the board can rest.

Risult. Let $\theta$ be the inclination of the board to the horizon; then $\tan _{2}^{\theta}=\mu$, where $\mu$ is the onefficient of the friction between the board and the sphere.

23. A string $P C B$ passes over a smooth pully $C$, and has a given weight $P$ attached to one extremity, while the other extremity $B$ is attached to one end of a heary uniform beam $A B$ at $B$. The other end $A$ of the beam rests upon a rough horizontal plane; determine the position of the beam when in equilibrium.

24. A hemisphere is supported by friction with its curved surface in contact with a horizontal and vertical plane; find the limiting position of equilibrim.

liesult. If $\theta$ be the inclination of the plane base to the horizon, $\sin \theta=\frac{8 \mu\left(1+\beta^{3}\right)}{3\left(1+\mu^{3}\right)}$.

25. When a person tries to pull out a two-handled drawer by pulling one of its handles in a direction perpendienlar to its front, find the condition under which the drawer will stick fast.

26. Ditermine the condition under which a given weight may be supported on a rough vertical screw withut the 
action of any force; for example, if the coefficient of friction be $\frac{1}{2}$, find the least number of turns which may be given to a thread on a cylinder 2 feet long and 6 inches in circumference.

Result. Eight.

27. Two uniform beams of the same length and material placed in a vertical plane, are in a state of rest bordering on motion under the following circumstances: their upper ends are connected by a smooth hinge, about which they can move freely; their other ends rest on a rough horizontal plane, and the beams are perpendicular to each other: find the coefficient of friction between the beams and the horizontal plane.

Result. $\mu=\frac{1}{2}$.

28. A straight uniform beam is placed upon two rough planes whose inclinations to the horizon are $\alpha$ and $\alpha^{\prime}$, and the coefficients of friction $\tan \lambda$ and $\tan \lambda^{\prime}$; shew that if $\theta$ be the limiting value of the angle of inclination of the beam to the horizon at which it will rest, $W$ its weight, and $R, R^{\prime}$ the pressures upon the planes

$$
2 \tan \theta=\cot \left(\alpha^{\prime}+\lambda^{\prime}\right)-\cot (\alpha-\lambda),
$$

$$
\frac{R}{\cos \lambda \sin \left(\alpha^{\prime}+\lambda^{\prime}\right)}=\frac{R}{\cos \lambda^{\prime} \sin (\alpha-\lambda)}=\frac{W}{\sin \left(\alpha-\lambda+\alpha^{\prime}+\lambda^{\prime}\right)} \text {. }
$$

29. A heavy right cylinder is placed with its base on a rough horizontal plane, and is capable of motion round its axis; find the least couple in a horizontal plane which will move it.

30. Two weights of different material are laid on an inclined plane connected by a string extended to its full length, inclined at an angle $\theta$ to the line of intersection of the inclined plane with the horizon; if the lower weight be on the point of motion find the magnitude and direction of the force of friction on the upper weight.

31. $\Lambda$ carriage stands upon four equal wheels; given the coefficient of friction between the axles and the wheels find the greatest slope on which it can remain at rest neglecting the weight of the wheels. 


\section{CHAPTER XI.}

\section{FIRXIBLE IXEXTESSIBLE STRISGS.}

182. A sтRINo is said to be perfectly flexible when any force, however mall, which is applied otherwise than along the direction of the string will change its form. For shortness, we use the word fexible as equivalent to perfectly Aexible. Sometimes the word chain is used as synonymous with string.

If a flexible string be kept in equilibrium by two forces, one at each end, wo assume as self-evident that those forces muat be equal and act in opposite directions, so that the string asumes the form of a straight line in the direction of the forces. In this case the tension of the string is measured by the force applied at one end.

Iet $A B C$ represent a string kept in equilibrium by a force $T$ at one end $A$ and an equal force $T$ at the other end $C$ acting in opposite directions along the line $A C$. Since any portion $A B$ of the string is in equilibrium it follows that a forec $T$ must act on $A \mathcal{B}$ at $B$ from $B$ towards $C$ in order to balance the force acting at $A$; and similarly, a force $T$ must act on $B C$ from $B$ towards $A$ in orler that $B C$ may be in equilibrium. This resule is expressed by saying that the tension of the string is the same throughoul.

Unless the contrary be cxpressed, a string is supposed incextensille and the boundary of a tranaverae section of it is supposed to be a curve, erery chord of which is indefinitely small.

153. When a fexible string is acted on by other fonces besides one at each end it may in equilibrium assume a curvilinear form. If at any point of the curve we suppose 
a section made by a plane perpendicular to the tangent, the mutual action of the portions on opposite sides of this plane must be in the direction of the tangent, or else equilibrium would not hold, since the string is perfectly flexible.

154. A heavy string of uniform density and thickness is suspended from two given points; required to find the equation to the curve in which the string hangs when it is in equilibrium.

Let $A, B$, be the fixed points to which the ends are attached; the string will rest in a rertical plane passing through $A$ and $B$, because there is no reason why it should deviate to one side rather than the other of this vertical plane. Let $A C B$ be the form it assumes, $C$ being the lowest point; take this as the origin of co-ordinates; let $P$ be any point in the curve; $C M$, which is vertical, $=y ; M P$, which is horizontal, $=x$; $C \ddot{P}=s$.

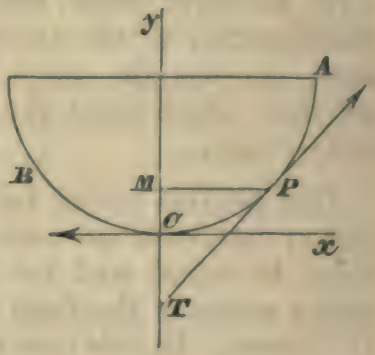

The equilibrium of any portion $C P$ will not be disturbed if we suppose it to become rigid. Let $c$ and $t$ be the lengths of portions of the string of which the weights equal the tensions at $C$ and $P$. Then $C P$ is a rigid body acted on by three forces which are proportional to $c, s$, and $t$, and act respectively, horizontally, vertically, and along the tangent at $P$. Draw $I^{\prime} T$ the tangent at $P$ meeting the axis of $y$ in $T$; then the forces holding $C P$ in equilibrium have their directions parallel to the sides of the triangle P.IIT, and therefore bear the same proportion one to another that these sides do (sec $\Lambda$ rt. 19); therefore

$$
\begin{aligned}
& \text { therefore } \quad \frac{d y}{d x}=\frac{8}{c} \text { and } \frac{d y}{d s}=\frac{s}{\sqrt{\left(c^{2}+s^{2}\right)}} \text {; } \\
& \text { therefore } \\
& \frac{P M I}{M T}=\frac{\text { tension at lowest point }}{\text { weight of the portion } C P} \text {, or } \frac{d x}{d y}=\frac{c}{8} \text {, } \\
& y+c=\sqrt{ }\left(c^{2}+8^{2}\right)
\end{aligned}
$$


the constant added being such that $y=0$ when $s=0$; thereforo

$$
d^{7}=y^{2}+2 y c
$$

1980

$$
\frac{d x}{d y}=\frac{c}{a}=\frac{c}{\sqrt{(y+2 y c)}} \text {, }
$$

therefore

$$
x=c \log \frac{y+c+\sqrt{\left(y^{2}+2 y c\right)}}{c} \text {. }
$$

the constant being chosan so that $x$ and $y$ vanish together. The last equation gives

$$
\alpha^{2}-y+0+\sqrt{ }\left(y^{2}+2 y c\right) \text {. }
$$

Transpose and sqgure; thus

$$
c^{3} e^{\frac{2}{2}}-2(y+c) e^{2}+e^{3}=0
$$

therefore

$$
y+c=d c\left(e^{i}+e^{-i}\right) \text {. }
$$

Also

$$
\begin{aligned}
& s=\sqrt{ }\left\{(y+c)^{2}-c^{2}\right\} \text { by } \\
& =\frac{1}{2} c\left(\dot{e}-e^{-i}\right) \text {. }
\end{aligned}
$$

Any one of these five equations may be taken as the equation to the curve. If in equation (4) we write $y$ for $y+c$, which amounts to moving the origin to a point vertically below the lowest point of the curve at a distance $c$ from it, we have

$$
y^{\prime}=10\left(e^{\circ}+e^{-\frac{0}{\sigma}}\right) \text {. }
$$

When the string is uniform in density and thickness, as in the present instance, the curre is called the common culenary.

185. To find the tension of the string at any point.

Iet the tension at $P$ be equal to the weight of a length $t$ of the string; then, as shewn in the last article,

$$
\frac{\text { tension at } P}{\text { weight of } C P}=\frac{P T}{T . M} \text {, therefure } \frac{t}{3}=\frac{d g}{d y} \text {. }
$$


But $s^{2}=y^{2}+2 y c$ by equation (2) of Art. 184, therefore

$$
t=y+c=y^{\prime} \text {. }
$$

This shews that the tension at any point is the weight of a portion of string whose length is the ordinate at that point, the origin being at a distance $c$ below the lowest point.

Hence, if a uniform string hang freely over any two points, the extremities of the string will lie in the same horizontal line when the string is in equilibrium.

186. To determine the constant $\mathrm{c}$, the points of suspension and the length of the string being given.

Let $A$ and $B$ be the fixed extremities, $C$ the lowest point of the curve.

$$
\begin{gathered}
O C=c, \quad O M=a, \\
O N=a^{\prime}, \quad M A=b, \\
N B=b^{\prime}, \quad C A=l, C B=l .
\end{gathered}
$$

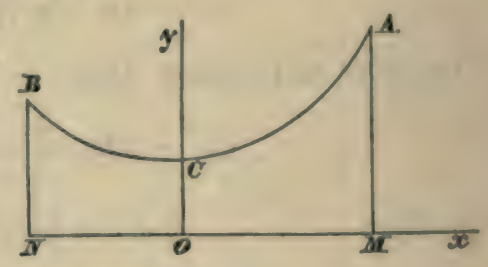

Also let

$$
\left.\begin{array}{l}
a+a^{\prime}=h \\
b-b^{\prime}=k \\
l+l^{\prime}=\lambda
\end{array}\right\} \ldots \ldots \ldots \ldots \ldots \ldots \ldots(1)
$$

then $h, k, \lambda$ are known quantities, since the length of the string and the positions of its ends are given. From Art. 184

$$
\left.\begin{array}{l}
b=\frac{1}{2} c\left(e^{\frac{a}{b}}+e^{-\frac{a}{b}}\right) \\
b^{\prime}=\frac{1}{2} c\left(e^{\frac{a}{a}}+e^{-\frac{a}{b}}\right) \\
l=\frac{1}{2} c\left(e^{\frac{a}{b}}-e^{-\frac{a}{b}}\right) \\
l^{\prime}=\frac{1}{2} c\left(e^{\frac{a}{b}}-e^{-\frac{a}{b}}\right)
\end{array}\right\}
$$

Fquations (1) and (2) are theoretically sufficient to enable us to eliminate $a, a^{\prime}, b, b^{\prime}, l$, and $l^{\prime}$ and to determine $c$. We may deduce from them

$$
\begin{aligned}
& \lambda=\frac{1}{2} c\left(e^{\frac{\alpha}{\sigma}}-e^{-\frac{a}{\sigma}}+e^{\frac{\alpha}{\theta}}-e^{-\frac{\alpha}{\sigma}}\right), \\
& k=\frac{1}{2} c\left(e^{\frac{\alpha}{0}}+e^{-\frac{a}{6}}-e^{\frac{\alpha}{\sigma}}-e^{-\frac{\alpha^{\circ}}{\sigma}}\right) ;
\end{aligned}
$$


thereforo

$$
\begin{aligned}
& \lambda+b=0\left(6^{\circ}-e^{-\frac{2}{6}}\right), \\
& \lambda-k=0\left(6^{\circ}-6^{-j}\right) \text {; } \\
& \lambda^{2}-b^{3}=c^{3}\left(e^{*}+e^{-\frac{40}{6}}-2\right) \\
& =\sigma^{2}\left(e^{\frac{2}{6}}+e^{-\frac{3}{2}}-2\right) \text {; } \\
& \sqrt{\left(\lambda^{2}-k^{2}\right)}=c\left(e^{2}-e^{-\frac{1}{2}}\right)
\end{aligned}
$$

therefore

therefore

This is the equation from which $c$ is to be found, but on secuunt of its tranacrendental form it can only be solved by approximation. If the exponents of e are small, we may expand by the exponential sheorem and thus obtain the approximate value of $c$. In order that the exponents may be small, $c$ must be large compared with $h$; since $\frac{d y}{d s}=\frac{a}{\sqrt{ }\left(c^{2}+s^{2}\right)}$ by Art. 184 it follows that when $c$ is large, compared with the length of the string, $\frac{d y}{d s}$ is small, and therefore the curve does not deriate much from a straight line. Hence, when the two points of support are nearly in a horizontal line and the distance between them nearly equal to the given length of the string, we may conclude that $\frac{h}{c}$ will be small. In this case, we have from (3)

$$
\sqrt{ }\left(\lambda^{2}-k^{2}\right)=2 c\left\{\frac{h}{2 c}+\frac{1}{[3}\left(\frac{h}{2 c}\right)^{2}+\frac{1}{[5}\left(\frac{h}{2 c}\right)^{3}+\ldots . .\right\}
$$

therefore $\quad \sqrt{ }\left(\lambda^{3}-h^{2}\right)=h+\frac{h^{3}}{24 c^{3}}$ approximately.

157. To find the equations of equilibrium achen a flexilile st ring is acted on by any furces.

Let $x, y, z$ be the co-ordinates of a point $P$ of the string; let s denote the length of the curve $B P$ mensured from some fixed print $B$ up to $P$, and $\delta$, the length of the are $I^{\prime} Q$ between $P$ and an adjacent point $Q$. Iet $\kappa$ be the area of a 
section of the string at $P$, and $\rho$ the density at $P$; let $T$ be the tension of the string at $P$; then $T \frac{d x}{d s}, T \frac{d y}{d s}$, and $T \frac{d z}{d s}$ are the resolved parts of $T$ parallel to the co-ordinate axes; and the resolved parts of the tension at $Q$ parallel to the axes will bé, by 'Taylor's 'Theorem,

$$
\begin{aligned}
& T \frac{d x}{d s}+\frac{d}{d s}\left(T \frac{d x}{d s}\right) \delta s+\text { terms in }(\delta s)^{2}, \& c ., \\
& T \frac{d y}{d s}+\frac{d}{d s}\left(T \frac{d y}{d s}\right) \delta s+\ldots \ldots \ldots \ldots \ldots \ldots \\
& T \frac{d z}{d s}+\frac{d}{d s}\left(T \frac{d z}{d s}\right) \delta s+\ldots \ldots \ldots \ldots \ldots . . . . . .
\end{aligned}
$$

Let $X_{\rho \kappa \delta s,} Y_{\rho \kappa \delta \delta}, Z \rho \kappa \delta s$ be the external forces which act on the element $P Q$ parallel to the axes. The equilibrium of the element will not be disturbed by supposing it to become rigid; hence, by Art. 27, the sum of the forces parallel to the axis of $x$ must vanish; thus

or

$$
\begin{gathered}
T \frac{d x}{d s}+\frac{d}{d s}\left(T \frac{d x}{d s}\right) \delta s+\ldots+X_{\rho \kappa \delta s}-T \frac{d x}{d s}=0, \\
\frac{d}{d s}\left(T \frac{d x}{d s}\right)+X_{\rho \kappa}=0 \text { ultimately. }
\end{gathered}
$$

Similarly

$$
\begin{aligned}
& \frac{d}{d s}\left(T \frac{d y}{d s}\right)+Y_{\rho \kappa}=0 \\
& \frac{d}{d s}\left(T \frac{d z}{d s}\right)+Z \rho \kappa=0 .
\end{aligned}
$$

and

The product $\kappa \rho$ may be conveniently replaced by $m$, so that if $m$ be constant $m l$ represents the mass of a length $l$ of the string, and therefore $m$ the mass of a unit of length of the string. If $m$ be not constant, conceive a string having its length equal to the unit of length and its section and density throughout the same as those of the given string at 
the point $(\kappa, y, z)$, and then $m$ will be the mans of such supposed string.

The element $\delta$ of the string, the equilibrium of which we have considered, becomes more nearly a particle the more we diminish $8 s$; hence it is sufficient to consider the three equations of Art. 27 instead of the six equations of Art. 73.

The three equations which we have found are theoretically sufficient for determining $T_{1} y$, and $z$ as functions of $x$, remembering that $\frac{d s}{d x}=\sqrt{ }\left\{1+\left(\frac{d y}{d x}\right)^{0}+\left(\frac{d z}{d x}\right)^{n}\right\}$; and when we know the values of $y$ and $z$ in terms of $x$, we know the equations to the curve which the string forms.

188. The equations for the equilibrium of a flexiblestring may be written thas;

$$
\left.\begin{array}{l}
T \frac{d^{2} x}{d s^{3}}+\frac{d T}{d s} \frac{d x}{d s}+m X=0 \\
T \frac{d^{2} y}{d s^{3}}+\frac{d T}{d s} \frac{d y}{d s}+m Y=0 \\
T \frac{d^{2} z}{d s^{2}}+\frac{d T}{d s} \frac{d z}{d s}+m Z=0
\end{array}\right\}
$$

Multiply these equations by $\frac{d x}{d s}, \frac{d y}{d s}$, and $\frac{d z}{d s}$ respectively and add; then, since

$$
\left(\frac{d x}{d s}\right)^{2}+\left(\frac{d y}{d s}\right)^{2}+\left(\frac{d s}{d s}\right)^{2}=1
$$

and

$$
\frac{d x}{d s} \frac{d^{2} x}{d s^{3}}+\frac{d y}{d s} \frac{d^{2} y}{d s^{3}}+\frac{d s}{d s} \frac{d^{2} s}{d s^{3}}=0 \text {, }
$$

we have $\frac{d T}{d s}+m\left(X \frac{d x}{d s}+Y \frac{d y}{d s}+Z \frac{d z}{d s}\right)=0 \ldots \ldots$ (2);

therefore $T+\int m\left(X \frac{d x}{d s}+Y \frac{d y}{d s}+Z \frac{d z}{d s}\right) d s=$ constant... (3). 
If the forces are such that $m(X d x+Y d y+Z d z)$ is the immediate differential of some function of $x, y, z$, as $f(x, y, z)$, then

$$
T+f(x, y, z)=\text { constant. }
$$

If the forees are such that their resultant at every point of the curve is perpendicular to the tangent at that point, we have

$$
X \frac{d x}{d s}+Y \frac{d y}{d s}+Z \frac{d z}{d s}=0,
$$

therefore, by (3), $T$ is constant.

In the equations (1) transpose the terms $m X, m Y, m Z$ to the right-hand side, then square and add; thus

$$
T^{2}\left\{\left(\frac{d^{2} x}{d s^{2}}\right)^{2}+\left(\frac{d^{2} y}{d s^{2}}\right)^{2}+\left(\frac{d^{2} z}{d s^{2}}\right)^{2}\right\}+\left(\frac{d T}{d s}\right)^{2}=m^{2}\left(X^{2}+Y^{2}+Z^{2}\right) \text {. }
$$

Hence if $\rho$ be the radius of absolute curvature of the curve formed by the string, and $F m \delta s$ the resultant external force on the element $\delta s$, so that $F^{2}=X^{2}+Y^{2}+Z^{2}$,

$$
\left(\frac{T}{\rho}\right)^{2}+\left(\frac{d T}{d s}\right)^{2}=m^{2} F^{2}
$$

If $T$ be constant $\frac{d T}{d s}=0$; hence in this case $m F$ varies as $\frac{1}{\rho}$. From the equations of equilibrium in $\Lambda$ rt. 187, we deduce by integration,

$$
\begin{aligned}
& T \frac{d x}{d s}=-\int m X d s, \\
& T \frac{d y}{d s}=-\int m Y d s, \\
& T \frac{d z}{d s}=-\int m Z d s .
\end{aligned}
$$

Square and add; then

$$
T^{s}=\left\{\int m X d s\right\}^{2}+\left\{\int m Y d s\right\}^{2}+\left\{\int m Z d s\right\}^{2} \ldots \ldots \ldots \text { (5). }
$$

The constants that enter when we integrate the differential equations of equilibrium must be determined from the special 
circumstances of each particular problem. Thus the coordinates of fixed points to which the ends of the string are attached may be given, and the length of the string.

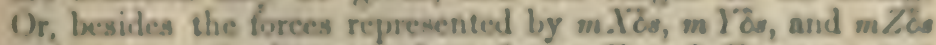
acting on each element, given forces $F_{0}$ and $F_{\text {a }}$ may act at the extremities of the string; in this ene if $T_{1}$ and $T$, denote the values of $T$ at the two extremities of the string, we must have $T_{1}$ equal in magnitude to $F_{2}$ and opposite to it in direction, and similarly for $T_{2}$ and $F_{8}$.

159. From equations (1) of Art. 188, eliminate $T$ and $\frac{d T}{d s}$; then we have

$$
\begin{gathered}
X\left(\frac{d^{2} y}{d s^{s}} \frac{d s}{d s}-\frac{d^{2} s}{d s^{2}} \frac{d y}{d s}\right)+Y\left(\frac{d s}{d s^{3}} \frac{d x}{d s}-\frac{d^{2} x}{d s^{3}} \frac{d s}{d s}\right) \\
+Y\left(\frac{d^{2} x}{d s^{3}} \frac{d y}{d s}-\frac{d^{2} y}{d s^{2}} \frac{d x}{d s}\right)=0
\end{gathered}
$$

this shews that the resultant external force which acts on an element $\delta_{s}$ of the string lies in the osculating plane at the point $(x, y, z)$.

190. The general equations of erpuilibrium become, when all the forces are in one plane, namely, that of $(x, y)$,

$$
\frac{d}{d s}\left(T \frac{d s}{d s}\right)+m X=0, \quad \frac{d}{d s}\left(T \frac{d y}{d s}\right)+m Y=0 \ldots \ldots(1) .
$$

Suppose $\boldsymbol{X}=0$, so that the external force is parallel to the axis of $y$; the tirst equation gives

therefore

$$
T \frac{d x}{d s}=\text { a constant, } C \text { say, }
$$

$$
T=\frac{C}{d x}
$$

Hense the second equation becomes

or

$$
\begin{aligned}
& C \frac{d}{d s}\left(\frac{d y}{d x}\right)+m Y=0, \\
& C \frac{d y}{d x} \frac{d x}{d s}+m Y=0
\end{aligned}
$$


For example; required the form of the curve when its weight is the only force acting on it, and the area of the section at any point is proportional to the tension at that point. Here $Y$ is constant and may be denoted by $-g$, the axis of $y$ being vertically upwards. And $T$ varies as $m$, so that $T=\lambda m$ where $\lambda$ is a constant. Thus from (2) and (3) we obtain

$$
\frac{d^{3} y}{d x^{3}}\left(\frac{d x}{d s}\right)^{2}=\frac{g}{\lambda} \text {. }
$$

Put $a$ for $\frac{\lambda}{g}$; thus $\frac{d^{2} y}{d x^{2}}\left(\frac{d x}{d s}\right)^{2}=\frac{1}{a}$,

therefore

$$
\frac{\frac{d^{2} y}{d x^{2}}}{1+\left(\frac{d y}{d x}\right)^{2}}=\frac{1}{a},
$$

therefore

$$
\tan ^{-1} \frac{d y}{d x}=\frac{x}{a}+\text { constant. }
$$

The constant vanishes if we suppose the origin at the lowest point of the curve; therefore

$$
\frac{d y}{d x}=\tan \frac{x}{a}
$$

therefore

$$
\frac{y}{a}=-\log \cos \frac{x}{a}
$$

Since in this case the area of the section at any point is proportional to the tension at that point, the curve determined by (4) is called the Catenary of equal strength.

Since $T=\lambda m=m a g$, we have the following result: the tension at any point is equal to the weight of a length $a$ of a uniform string of the same area and density as the string actually has at the point considered.

191. The equations (1) of the preceding Article may be written

$$
\begin{aligned}
& T \frac{d^{2} x}{d s^{2}}+\frac{d T}{d s} \frac{d x}{d s}+m X=0 \\
& T \frac{d^{2} y}{d s^{2}}+\frac{d T}{d s} \frac{d y}{d s}+m Y=0
\end{aligned}
$$


Multiply (1) by $\frac{d y}{d s}$ and (2) by $\frac{d x}{d s}$ and subtract ; thus

$$
T\left(\frac{d x}{d s^{2}} \frac{d y}{d s}-\frac{d y}{d s^{2}} \frac{d x}{d s}\right)+m\left(X \frac{d y}{d s}-Y \frac{d x}{d s}\right)=0,
$$

from which, aince $\frac{d x}{d s} \frac{d^{2} x}{d s^{3}}+\frac{d y}{d s} \frac{d^{2} y}{d s^{3}}=0$, we find

$$
T=\frac{m \frac{d x}{d s}}{\frac{d y}{d s^{\prime}}}\left(x \frac{d y}{d x}-Y \frac{d x}{d s}\right)
$$

A gain, multiply (1) by $\frac{d x}{d s}$ and (2) by $\frac{d y}{d s}$ and add; then

$$
\frac{d T}{d s}+m\left(X_{d s}^{d x}+Y \frac{d y}{d s}\right)=0
$$

Frum (3) and (4) by eliminating $T$, we deduce

$$
m\left(X \frac{d x}{d s}+Y \frac{d y}{d s}\right)+\frac{d}{d s}\left\{\frac{m \frac{d x}{d s}}{\frac{d^{2} y}{d s^{2}}}\left(X \frac{d y}{d s}-Y \frac{d x}{d s}\right)\right\}=0,
$$

which is the general equation to the curve when given forces act in one plane.

192. In $\Lambda$ rt. 188 we have found the equations

$$
\begin{array}{r}
\frac{d T}{d s}+m\left(X \frac{d x}{d s}+Y \frac{d y}{d s}+Z \frac{d z}{d s}\right)=0 \ldots \ldots \ldots(1), \\
\left(\frac{T}{\rho}\right)^{2}+\left(\frac{d T}{d s}\right)^{\prime}=m^{2} F^{s} \ldots \ldots \ldots \ldots \ldots \ldots(2) .
\end{array}
$$

Let $\phi$ be the angle which the resultant force $m F$ ds makes with the tangent at the point $(x, y, z)$; then

$$
F \cos \phi=X \frac{d x}{d s}+Y \frac{d y}{d s}+Z \frac{d s}{d s}
$$


therefore, by (1),

$$
\frac{d T}{d s}=-m F \cos \phi
$$

and therefore, by (2),

$$
\left(\frac{T}{\rho}\right)^{3}=m^{2} F^{2} \sin ^{2} \phi \ldots \ldots \ldots \ldots \ldots \ldots \text { (4). }
$$

If the force be such that its direction always passes through a fixed point, the whole string will lie in a plane passing through its ends and through the fixed point, for there is no reason why it should lie on one side rather than the other of this plane. Let $r$ be the distance of the point $(x, y, z)$ of the curve from the fixed point, $p$ the perpendicular from the fixed point on the tangent at $(x, y, z)$; then (3) and (4) may be written

$$
\begin{gathered}
\frac{d T}{d s}=-m F \frac{d r}{d s} \\
\frac{T}{\rho}=m F \frac{p}{r} \cdots .
\end{gathered}
$$

Hence

$$
\frac{1 d T}{T d s}=-\frac{r d r}{\rho p} \frac{d s}{d s}=-\frac{1}{p} \frac{d p}{d s} ;
$$

therefore

$$
\log T=\text { constant }-\log p \text {, }
$$

or

$$
T_{p}=C .
$$

Also, from (5),

$$
T=-\int m F d r .
$$

Therefore

$$
\frac{C}{p}=-\int m F d r \text {. }
$$

Put $\phi(r)$ for $-\int m F d r$; then

$$
\phi(r)=\frac{C}{p}=C\left\{\frac{1}{r^{2}}+\frac{1}{r^{4}}\left(\frac{d r}{d \theta}\right)^{2}\right\}^{\frac{3}{2}},
$$

and from this differential equation the relation between $r$ and $\theta$ must be found.

The equation $T_{p}=C$ may also be obtained simply thus: suppose a finite portion of the string to become rigid; this 
portion is actod on by the tensions at its two ends and by other forces which all juss throwgh a fired point; take moments round this fixed point; hence the product of the tension into the perpendicular from the fixed point on the tangent must have the same value at the two ends of the finite portion of the string. Thus $T p=$ constant.

193. The results of the last Article give ns the form of a string when acted on by any central force; these results may also be obtained directly in the following manner.

Let $O$ be the centre of force, $P$ a point in the curve, $Q$ an

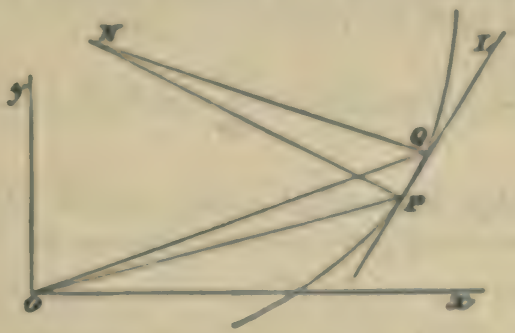

adjacent point; $r, \theta$ the polar co-ordinates of $P$; let $s$ be the length of the curve measured from some fixed point up to $P$, and $P^{\prime} Q=\delta 8$. Draw $P L$ the tangent at $P$; and $P ., Q N$ normals at $P$ and $Q$ respectively, then $P N$ is ultimately the radius of currature at $P$. Fet $T$ denote the tension at $P, T+\delta T$ the tension at $Q, F$ im ss the force acting on the element $P Q$, which will ultimately be in the direction $O P$ produced.

Iet $P N Q=\psi$, and $\phi$ be the angle between $P I$, and $O P$ produced. Resolve the forces acting on the element along $P L$ and $P N$; then

$$
\begin{gathered}
(T+\delta T) \cos \psi+F i n \delta \cos \phi-T=0, \\
(T+\delta T) \sin \psi-F i m \delta \sin \phi=0 .
\end{gathered}
$$

Now

$$
\sin \psi=\frac{\delta s}{\rho} \text { ultimately, and } \cos \psi=1 \text {. }
$$


IIence the equations become

or

$$
\delta T+F m \delta s \cos \phi=0,
$$

$$
\frac{d T}{d s}+F m \cos \phi=0 \text {, }
$$

and

$$
\frac{T}{\rho}-F m \sin \phi=0,
$$

and the solution may be continued as in the last Article.

We have supposed the force repulsive, that is, tending from $O$; if it act towards $O$ the figure will be convex towards $O$ and we shall have the results

$$
\frac{d T}{d s}-m F \cos \phi=0, \quad \frac{T}{\rho}-m F \sin \phi=0 .
$$

194. A string is stretched over a smooth plane curve; to find the tension at any point and the pressure on the curve.

First suppose the weight of the string neglected.

Let $A P Q B$ be the string, $A$ and $B$ being the points where

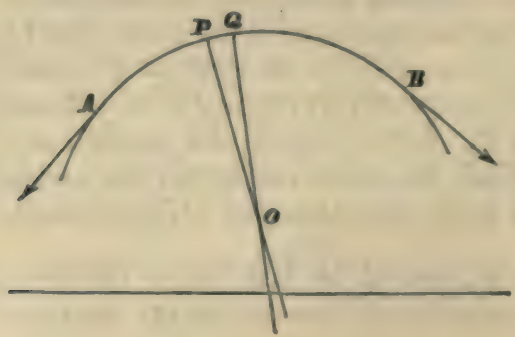

it leaves the curve. Iet $P, Q$ be adjacent points in the string; let the normals to the curve at $P$ and $Q$ meet at $O$; let $\theta$ be the angle which $P O$ makes with some fixed straight line, and $\theta+\delta \theta$ the angle which $Q O$ makes with the same line. The element $P Q$ is acted on by a tension at $P$ along the tangent at $P$, a tension at $Q$ along the tangent at $Q$, and the resistance of the smooth curve which will be ultimately along $P O$. 
Let $s$ be the length of the curve measured from some fixed point up to $R$, and $P Q=\delta$; let Ribs denote the resistance of the curve on $P Q, T$ the tension at $I, T+\delta T$ the tenaion at $Q$. Suppose the element $P Q$ to become rigid, and resolve the forces acting on it along the tangent and normal at $P$; then

$$
\begin{aligned}
T-(T+\delta T) \cos \delta \theta & =0 . \\
R \hat{s} s-(T+\delta T) \sin \delta \theta & =0 .
\end{aligned}
$$

Now

$$
\cos \delta \theta=1-\frac{(8 \theta)^{2}}{1.2}+\frac{\left(8 \theta^{\circ}\right.}{4}-\ldots
$$

bence (1) gives by division by $\delta \theta$

$$
\frac{\delta T}{\delta \theta}-(T+\delta T)\left\{\frac{\delta \theta}{2}-\frac{(\delta \theta)^{3}}{L}+\ldots\right\}=0 ;
$$

therefore ultimately

$$
\frac{d T}{d \theta}=0,
$$

therefore

$$
T=\text { constant }
$$

Also $\delta s=\rho \delta \theta$ ultimately, $\rho$ being the radius of curvature at $P$, therefore, from (2), we have

$$
R=\frac{T}{\rho}
$$

Since $T$ is constant, the string will not be in equilibrium unless the forces pulling at its two ends are equal; this is usually assumed as self-erident in the theory of the pully.

The uhole pressure on the curve will be $\int R d s$; therefore by (1), the whole pressure

$$
=\int \frac{T}{\rho} d \theta=\int T d \theta
$$

Since $T$ is constant, $\int T i d \theta=T \theta+$ constant ;

therefore the whole pressure $=T\left(\theta_{3}-\theta\right)$, supposing $\theta_{4}$ the value of $\theta$ at $A$, and $\theta_{2}$ the value of $\theta$ at $B$. 
Next suppose the weight of the string taken into account.

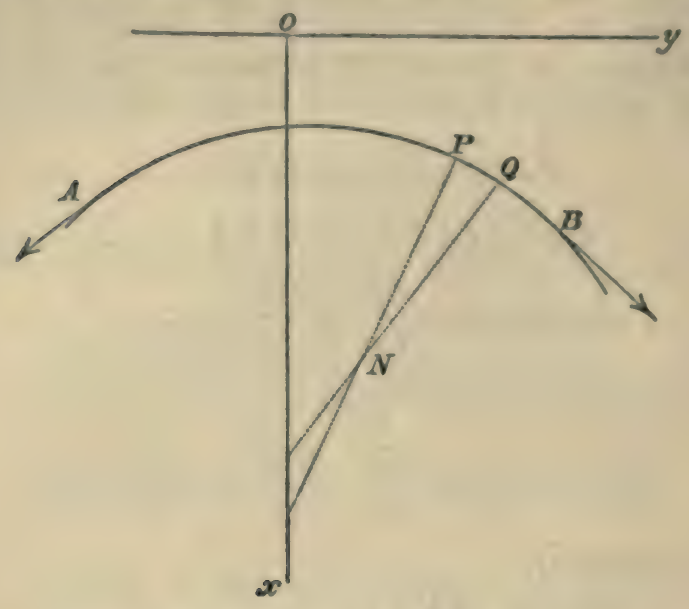

Take the axis of $y$ horizontal and that of $x$ vertically downwards. The element $P Q$ is acted on by a tension at $P$ along the tangent at $P$, a tension at $Q$ along the tangent at $Q$, the weight of the element vertically downwards, and the resistance of the smooth curve which will be ultimately along the normal at $P$. Let $\theta$ be the acute angle which the normal $P N$ makes with the axis of $x, \theta+\delta \theta$ the angle which the mormal $Q N$ makes with the axis of $x$. Let $s$ be the length of the curve measured from some fixed point up to $P$, and $P Q=\delta s$; let $T$ be the terision at $P$, and $T+\delta T$ the tension at $Q$; let $m g \delta s$ be the weight of the element, and $R \delta s$ the resistance of the smooth curve on the element. Suppose the element $P Q$ to become rigid, and resolve the forces acting on it along the tangent and normal at $P$; then

$$
\begin{aligned}
T-(T+\delta T) \cos \delta \theta-m g \delta s \sin \theta & =0 \ldots \ldots \ldots \ldots(5) . \\
R \delta s-(T+\delta T) \sin \delta \theta-m g \delta s \cos \theta & =0 \ldots \ldots \ldots \ldots(6) .
\end{aligned}
$$

From (5) we obtain ultimately

$$
\frac{d T}{d s}=-m g \sin \theta
$$


and from (6)

$$
I 6=\frac{T}{\rho}+m g \cos \theta
$$

where $\rho$ is the radius of curvature of the curve at $I$.

Since the curve is supposed to be a known curve, sand $\rho$ may be supposed known functions of $\theta$; thus $(\overline{7})$ and $(8)$ will enable us to find $T$ and $I$ in terms of $\theta$. Or we may express $T$ and $R$ in terms of the rectangular co-ordinates of the point $P$; for if we denote these co-ordinates by $x$ and $y$, we have

$$
\sin \theta=\frac{d x}{d s}, \quad \cos \theta=\frac{d y}{d s}
$$

thus (i) may be written

$$
\frac{d T}{d s}=-m g \frac{d s}{d s} ;
$$

therefore, if $m$ be constant,

$$
T=-m g x+C,
$$

where $C$ is some constant; the value of this constant will be known if the tension of the string be known at some given point, for example at $A$ or at $B$.

Also from (8)

$$
R=\frac{C-m g x}{\rho}+m g \frac{d y}{d s}
$$

and $\rho$ and $\frac{d y}{d s}$ will be known in terms of $x$ and $y$ since the curve is known.

In the above investigations we stated that the resistance of the curve on the element $P Q$ acts ullimately along the normal at $P$; and in forming the equations of equilibrium of the element of the string we supposed the resistance to act strictly along the normal at $P$. It is essy to shew that no error is thus introduced. For the resistance at $P$ is along the normal at $P$, and at $Q$ it is along the normal at $Q$, hence 
the resistance on the element $F Q$ may be taken to be a force which acts in some direction intermediate between the directions of these two normals; suppose $\psi$ the angle which its direction makes with that of the normal at $P$. We should then write $l i \delta s \cos \psi$ instead of $R \delta s$ in the equations (2) and $(6)$, where $\psi$ is an angle less than $\delta \theta$; hence in the limit $\cos \psi=1$ and equations (4) and (8) remain unchanged. Also the term $R \delta s \sin \psi$ must be introduced into equations (1) and (5); thus equation (1) becomes

$$
T-(T+\delta T) \cos \delta \theta-R \delta s \sin \psi=0 ;
$$

therefore $\frac{\delta T}{\delta \theta}-(T+\delta T)\left\{\frac{\delta \theta}{2}-\frac{(\delta \theta)^{3}}{L 4}+\ldots\right\}+R \frac{\delta s}{\delta \theta} \sin \psi=0$; and ultimately $\frac{\delta s}{\delta \theta}=\rho$ and $\sin \psi=0$; hence as before

$$
\frac{d T}{d \theta}=0 .
$$

Similarly we may shew that equation (7) remains true after the introduction of the term $R \delta s \sin \psi$ into equation (5).

195. A string is stretched over a rough plane curve; to find the tension at any point and the pressure on the curve in the limiting position of equilibrium.

First suppose the weight of the string neglected. See the first figure of Article 194.

The element $P Q$ is acted on by a tension at $P$ along the tangent at $P$, a tension at $Q$ along the tangent at $Q$, the resistance of the curve which will be ultimately along the normal at $P$, and the friction which will be ultimately along the tangent at $P$ and in the direction opposite to that in which the element is about to move. Let $T$ denote the tension at $P, T+\delta T$ that at $Q, R \delta s$ the resistance, $\mu R \delta s$ the friction; suppose the string about to move from $A$ towards $B$. Suppose the element $P Q$ to become rigid, and resolve the forces acting on it along the tangent and normal at $P$; then

$$
\begin{aligned}
T+\mu R \delta s-(T+\delta T) \cos \delta \theta & =0 \ldots \ldots \ldots(1), \\
R \delta s-(T+\delta T) \sin \delta \theta & =0 \ldots \ldots \ldots \ldots(2) .
\end{aligned}
$$


From (1) we have ultimately

$$
\frac{d T}{d s}=\mu R
$$

and from (2).

$$
\frac{T}{\rho}=\mathbb{R}
$$

therefore

$$
\frac{\rho}{T} \frac{d T}{d s}=\mu, \text { or } \frac{1}{T} \frac{d T}{d \theta}=\mu,
$$

therefore

$$
\log T=\mu \theta+\text { constant, }
$$

therefore

$$
T=C 0^{\circ} \text {. }
$$

Let $T$, be the force which acts on the string at the end $A$, and therefore the value of $T$ at this point; and let $T_{z}$ be the force at $B_{i}$ let $\theta_{1}$ and $\theta_{2}$ be the corresponding values of $\theta_{\text {; }}$

then

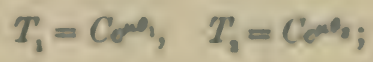

therefore

$$
T_{i}=e^{(0,-0,1)}
$$

and

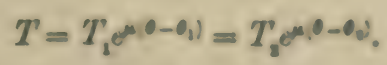

Also

$$
\begin{aligned}
\int R d s & =\int \frac{T}{\rho} d s=\int T d \theta=T_{s} \int \theta^{\left(\theta-\theta_{1}\right)} d \theta, \\
& =\frac{T}{\mu} \sigma^{\left(\theta-\theta_{1}\right.}+\text { constant } ;
\end{aligned}
$$

therefore the whole pressure on the curve

$$
=\frac{T_{e} e^{-\omega_{1}}}{\mu}\left(\omega_{0}-e^{-\omega_{1}}\right)=\frac{T_{2}-T_{1}}{\mu} .
$$

Next suppose the weight of the string taken into account. Proceeding as in the second case of Art. 194, and supposing the string about to move from $A$ to $B$, we have

$$
\begin{aligned}
T-\left(T+\delta T^{\prime}\right) \cos \delta \theta-m g \delta \theta \sin \theta+\mu R \delta s & =0 \ldots \ldots(3) . \\
R \delta s-(T+\delta T) \sin \delta \theta-m g \delta \cos \theta & =0 \ldots \ldots(6) .
\end{aligned}
$$


From (5) we obtain ultimately

$$
\frac{d T}{d s}=\mu R-m g \sin \theta,
$$

and from (6)

$$
R=\frac{T}{\rho}+m g \cos \theta ;
$$

therefore

$$
\frac{d T}{d s}=\frac{\mu T}{\rho}+m g(\mu \cos \theta-\sin \theta),
$$

therefore

$$
\rho \frac{d T}{d s}-\mu T=m g(\mu \cos \theta-\sin \theta) \rho,
$$

that is

$$
\frac{d T}{d \theta}-\mu T=m g(\mu \cos \theta-\sin \theta) \rho .
$$

Thus we have a differential equation for finding $T$, and we may proceed in the ordinary way to obtain the solution. Multiply both sides of the last equation by $e^{-\mu \theta}$; thus

$$
\frac{d}{d \theta}\left(T e^{-\mu \theta}\right)=m g e^{-\mu \theta}(\mu \cos \theta-\sin \theta) \rho ;
$$

therefore $T e^{-\mu \theta}=\int m g e^{-\mu \theta}(\mu \cos \theta-\sin \theta) \rho d \theta$.

IIence when $\rho$ is known in terms of $\theta$ we shall only have to integrate a known function of $\theta$ in order to obtain the value of $T$ in terms of $\theta$.

196. To form the equations of equilibrium of a string stretched over a smooth surface and acted on by any forces.

Let $s$ be the-length of the string measured from some fixed point $B$ to the point $P ; x, y, z$ the co-ordinates of $P ; \delta s$ the length of the element of the string between $P$ and an adjacent point $Q ; m \delta s$ the mass of the element; $R \delta s$ the resistance of the surface on this element, the direction of which will be ultimately the normal to the surface at $P$; let $\alpha, \beta, \gamma$ be the angles which the normal at $P$ makes with the axes; $X m \delta s$, $Y m \delta s, Z m \delta s$ the forces parallel to the axes acting on the element, exclusive of the resistance $R \delta$ s. Hence, in the equa- 
tions of Art. 187, for $X_{m}$ we must put $X_{m}+R \cos \alpha_{1}$ and make similar substitutions for $I_{m} m$ and $Z m$; therefore

$$
\begin{aligned}
& \frac{d}{d s}\left(T \frac{d t}{d s}\right)+X m+R \cos \alpha=0 \\
& \frac{d}{d s}\left(T \frac{d y}{d s}\right)+I m+R \cos \beta=0 \\
& \frac{d}{d s}\left(T \frac{d s}{d s}\right)+Z m+R \cos \gamma=0
\end{aligned}
$$

Multiply (1) by $\frac{d x}{d s}$, (2) by $\frac{d y}{d s}$, and (3) by $\frac{d z}{d s}$, and add; then, since

$$
\frac{d x}{d s} \cos \alpha+\frac{d y}{d s} \cos \beta+\frac{d s}{d s} \cos \gamma=0,
$$

because a tangent to the surface at any point is perpendicular to the normal at that point, we have, as in $\mathbf{A r t}$. 158,

$$
\frac{d T}{d s}+m\left(x \frac{d x}{d s}+Y \frac{d y}{d s}+Z \frac{d s}{d s}\right)=0
$$

A main, multiply (1) by $\cos \alpha$, (2) by $\cos \beta$, and (3) by $\cos \gamma$, and aild; then $T\left\{\frac{d^{2} x}{d s^{2}} \cos \alpha+\frac{d^{2} y}{d s^{2}} \cos \beta+\frac{d^{2} z}{d s^{2}} \cos \gamma\right\}$

$$
+m\{X \cos \alpha+Y \cos \beta+Z \cos \gamma\}+R=0 \ldots \text { (5). }
$$

Let Fnos be the result.unt of Ximose, Imôs, Zmôs, and $\psi$ the angle its direction makes with the normal to the surface at the point $(x, y, z)$; then

$$
X \cos \alpha+Y \cos \beta+Z \cos \gamma=F \cos \psi .
$$

Iat $\rho$ be the ralius of absolute curvature of the curve formed by the string at the point $(x, y, z) ; \alpha, \beta, \gamma$ the angles its T. S. 16 
direction makes with the axes; $\phi$ the angle its direction makes with the normal to the surface; then

$$
\frac{d^{2} x}{d s^{2}}=\frac{\cos \alpha^{\prime}}{\rho}, \quad \frac{d^{2} y}{d s^{2}}=\frac{\cos \beta}{\rho}, \quad \frac{d^{2} z}{d s^{2}}=\frac{\cos \gamma^{\prime}}{\rho} .
$$

Hence (5) becomes

$$
\frac{T}{\rho} \cos \phi+F m \cos \psi+R=0
$$

Let $u=0$ be the equation to the surface; then

$$
\frac{\cos \alpha}{\frac{d u}{d x}}=\frac{\cos \beta}{\frac{d u}{d y}}=\frac{\cos \gamma}{\frac{d u}{d z}}=N \text { suppose. }
$$

Hence (1) may be written

$$
T \frac{d^{2} x}{d s^{2}}+X m+\frac{d T}{d s} \frac{d x}{d s}+R N \frac{d u}{d x}=0,
$$

and (2) and (3) may be similarly expressed.

Eliminate $\frac{d T}{d s}$ and $R N$, and we obtain

$$
\begin{aligned}
\left(T \frac{d^{2} x}{d s^{2}}+X m\right)\left(\frac{d y}{d s}\right. & \left.\frac{d u}{d z}-\frac{d z}{d s} \frac{d u}{d y}\right) \\
& +\left(T \frac{d^{2} y}{d s^{3}}+Y m\right)\left(\frac{d z}{d s} \frac{d u}{d x}-\frac{d x}{d s} \frac{d u}{d z}\right) \\
& +\left(T \frac{d^{2} z}{d s^{2}}+Z m\right)\left(\frac{d x}{d s} \frac{d u}{d y}-\frac{d y}{d s} \frac{d u}{d x}\right)=0 .
\end{aligned}
$$

If we put for $T$ its value from (4), the resulting equation, together with $u=0$, will determine the curve formed by the string.

It appears from $\Lambda$ rt. 189 that the resultant of $F_{m} \delta s$ and $R \delta s$ must lie in the osculating plane of the curve at the point 
$(x, y, z)$. If the direction of Fimôs be always normal to the surface $u=0$, then, since that of $R \delta \delta$ is also normal to the surface, it follows that the normal to the surfice lies in the asculatim plane to the curve. This we know to be a property of the lines of maximum or minimum length that can be drawn on a surface between two given points. Hence, when a string is stretebod over a smooth surlace and acted on only by fores which are in the directions of normals to the surface at their points of application, it forms the line of maximum or minimum length that can be drawn on the surface between the extreme points of its contact with the surface.

When tines is always normal to the surface, it follows from (1) that $T$ is constint.

197. We will now give some miscellaneous theorems connocted with the subject of Alexible strings.

1. Required the alsecisen of the centre of pravity of an assignod portion of any string at rest, supposing its ends fixed, and gravity the only force.

This may be obtained by the ordinary process of integration, or more simply in the following maner. Imagine any portion of the string to become rigid: then it is kept in equilibrium by its weight and the tensions at the ends; these trinsions act in the directions of the tangents at the ends. Hence the centre of gravity of any portion must be vertically over the point of intersection of the tangents at the extremities of the portion.

II. Suppose in Art. 192 that the string is uniform, and that the force is attractive, and varies as the $n^{\text {th }}$ power of the distance. Thus we may put $F=-\mu r^{\circ}$; therefore

$$
\frac{C}{p}=\frac{m \mu r^{n+n}}{n+1}+\text { constant. }
$$

In the particular case in which the constant here introduced is zero we can easily complete the solution of the problem. We have

$$
\frac{1}{p}=\frac{r^{m}}{\lambda}
$$

where $\lambda$ is put for $\frac{C(n+1)}{w \mu}$. 
Put $\frac{1}{u}$ for $r$; then

therefore

$$
u^{3}+\left(\frac{d u}{d \theta}\right)^{2}=\frac{1}{\lambda^{2} u^{2 n+s}},
$$

therefore

$$
\left(\frac{d u}{d \theta}\right)^{2}=\frac{1-\lambda^{2} u^{2 n+4}}{\lambda^{2} u^{2 n+2}},
$$

$$
\frac{d \theta}{d u}=\frac{\lambda u^{n+1}}{\sqrt{\left(1-\lambda^{2} u^{2 n+f}\right)}} .
$$

Therefore, by integration,

$$
(n+2) \theta+\text { const. }=\sin ^{-1}\left(\lambda u^{n+2}\right) \text {; }
$$

therefore

$$
\frac{\lambda}{r^{n+3}}=\sin \{(n+2) \theta+\text { constant }\} \text {. }
$$

If we fix the position of the initial line so that $r$ may have its least value where $\theta=0$, we shall determine the constant, and obtain

$$
r^{n+2}=\lambda \sec (n+2) \theta ;
$$

or denoting by $a$ the value of $r$ when $\theta=0$,

$$
r^{n+2}=a^{n+2} \sec (n+2) \theta .
$$

III. Suppose a flexible string to be in equilibrium under the action of a central force. Imagine any portion of the string to become rigid: then it is kept in equilibrium by the tensions at the ends and the resultant of the action of the central force on the elements of the string; this resultant will be some single force acting through the centre of force. Thus the portion of the string may be considered to be in equilibrium under the action of three forces; and these forces will therefore meet at a point. Hence we obtain the following theorem: The resultunt action of the central force on any portion of the string is directed along the straight line which joins the centre of force with the point of intersection of the tangents at the ends of the portion. 
IV. Suppose that a flexible string is in equilibrium nnder the action of a eentral force which varies as the distance. Let $r$ be the distance of any point from the centre of force; $x, y$ the co-arinates of the point referred to axes having the centre of force as origin. 1et the force on an element of the string of which the length is de and mass mos situated at the point $\{x, y\}$ be $\mu r m c i s$ : then this force can be resolved into pxmsa and $\mu y m c$ parallel to the axes of ar and $y$ respectively.

Hence the components, parallel to the axes of $x$ and $y$, of the action of the central force on any portion of the string are $\mu$ frowds and $\mu$ fymeds respectively, the integrations extending over the portion considered. Now if $\vec{x}$ and $y$ le the co-ordinates of the centre of gravity of the portion, we have

$$
\bar{x}=\frac{\int x m d s}{\int m d s}, \bar{y}=\frac{\int y m d s}{\int m a d s} .
$$

Hence we obtain the following theorem: The straight line which joins the centre of grarity of any portion of the string to the centre of furce coincides with the dircetion of the resultans central furce on the portion.

Hence combining this theorem with that obtained in III. we obtain the following property of the flexible string which is in equilibrium under the action of a central force varying as the distance: The centre of gravity of any portion lies on the straight line which joins the centre of force arith the point of intersection of the tangents at the extremitics of the portion.

Thus by II. we see that the property here enunciated will hold for a uniform string in the form of the curve

$$
r=a^{3} \sec 30 .
$$

V. Two weights sre connected by a string which passes over a rough horizontal cylinder in a plane perpendicular to the axis: it is required io determine the resultant of the normal actions between the string and the cylinder in the state bordering on motion.

The normal action on any element \&s of the string may bo denoted by $R \delta$ s, and the friction on the element by $\mu$ lice; thus the friction on the element kears a constant ratio to the normal action, and the directions of the turo forces are at right angles. 
Let $P$ be the resultant normal action, and suppose its direction to make an angle $\theta$ with the vertical; then the resultant friction will be $\mu P$, and its direction will make an angle $\frac{\pi}{2}-\theta$ with the vertical on the other side of it. Ilence, supposing the string to become rigid, and resolving horizontally,

$$
P \sin \theta-\mu P \sin \left(\frac{\pi}{2}-\theta\right)=0 .
$$

$\Lambda$ gain, resolving vertically, and denoting by $W$ the sum of the weights of the system which hangs over the cylinder, we have

$$
P \cos \theta+\mu P \cos \left(\frac{\pi}{2}-\theta\right)-W=0 .
$$

Hence we obtain $\tan \theta=\mu$,

$$
P=W \cos \theta=\frac{W}{\sqrt{ }\left(1+\mu^{2}\right)} .
$$

VI. Suppose a heavy string which is not of uniform density and thickness to be suspended from two fixed points, and to be in equilibrium. Let $t$ be the tension at any point, $\theta$ the angle which the tangent at that point makes with the horizon; then $t \cos \theta$ will be constant. For imagine any portion of the string to become rigid, then the only horizontal forces which act on it are the resolved parts of the tensions at each end; and these must therefore be equal in magnitude: therefore

$$
t \cos \theta=\text { constant }=\tau \text { suppose............. (1). }
$$

Let $w$ be the weight of the portion of the string contained between any fixed point and the variable point considered. Then by resolving the forces vertically we obtain in a similar manner

therefore

$$
\begin{aligned}
& t \sin \theta-w=\text { constant } \\
& w=\tau \tan \theta+\text { constant ............... (2). }
\end{aligned}
$$

$\Lambda$ gain, proceeding as in $\Lambda \mathrm{rt}$. 193, that is resolving the forces which act on an element along the normal, we find

$$
\frac{t}{\rho}-g m \cos \theta=0
$$


where gmis is taken as the weight of the element is. Honere, frum (i) and (3),

$$
\frac{T}{\rho}=g n \cos ^{\circ} \theta \text {. }
$$

\section{EX.MPLES.}

1. In the enmmon catenary shew that the weight of the string between the lowest print and any other point is the germetrical mean between the sum and difference of the tensions at the two puints.

2. If $\alpha$ and $\beta$ are the inclinations to the horizon of the tangents at the extremities of a portion of a common eatrnary, and $t$ the length of the portion, shew that the beight of one extremity above the other is

$$
l \frac{\sin \frac{a+\beta}{2}}{\cos \frac{\alpha-\beta}{2}}
$$

the portion is supposed to be all on the same side of the lowest point.

3. A uniform heavy chain 110 feet long is suspended from two points in the same horizontal plane 108 feet asumder; shew that the tension at the lowest point is $\mathbf{1 . 4 7 7}$ times the weight of the chain nearly.

4. A uniform chain of length $2 l$ is suspended from two fixerl points in the sume horizontal plane; $2 a$ is the distance between the fixed points and $c$ the length of chain whose weight is equal to the tension at the lowest point; shew that when $l$ is snch that the tension at the points of suspension is the least possible that tension is equal to the weight of a length $\frac{a l}{c}$ of the chain, and $l$ and $e$ are determined by

$$
l=\frac{d}{2} c\left(a^{2}-a^{-\frac{2}{0}}\right), \quad\left(l^{2}+c^{2}\right) c^{2}=a^{2} l^{2} .
$$


5. If a uniform chain be fixed at two points, and any number of links $A, B, C, \ldots$ be at liberty to move along smooth horizontal lines in the same vertical plane, prove that the loops $A B, B C, C D, \ldots$ will form themselves into curves which will all be ares of the same catenary.

6. Three links of a chain $A, B$, and $C$ are moveable freely along three rigid horizontal straight lines in the same vertical plane. If when $A$ and $C$ are pulled as far apart as possible, their horizontal distances from $B$ are equal, shew that this will always be the case when they aro held in any other position.

7. A chain hangs in equilibrium over two smooth points which are in a horizontal straight line and at a given distance apart; find the least length of the chain that equilibrium may be possible.

Result. The least length is $h e$, where $h$ is the given distance.

8. Prove that the exertion necessary to hold a kite diminishes as the kite rises higher, the force of the wind being independent of the height, and the pressure of the wind on the string being neglected.

9. A uniform heary string rests on an are of a smooth curve whose plane is vertical, shew that the tension at any point is proportional to its vertical height above the lowest point of the string. If the string rests on a parabola whose axis is vertical, determine the vertical distance of its ends below the highest point so that the pressure at this point may be equal to twice the weight of a unit of length of the string.

Result. The vertical distance is equal to half the latus rectum of the parabola.

10. One end of a uniform heavy chain hangs freely over the edge of a smooth table, and the other end passing over a fixed pully reaches to the same distance below the table as the pully is above it. Supposing half the chain to be on the table in the position of equilibrium, compare its whole length with the height of the pully.

Result. 'The length is to the height as $6+2 \sqrt{ } 3$ is to 1 . 
11. A uniform heavy chain is fastened at its extremitien (1) two rings of efual weights which slite on smooth reds intersecting in a vertical plane and inclined at the rame angle a to the rertical; find the condition that the tension at the lowest point may be equal to half the weight of the chain; and in that case shew that the vertical distance of the rings from the point of intersection of the rods is

$$
\frac{l}{2} \cot \alpha \log (1+\sqrt{2}),
$$

where $l$ is the length of the chain.

12. The density at any point of a catenary of variable density varies as the radius of curvature; deternine the equation to the catenary.

Result. The curve in Art. 190.

13. A heavy cord with one end fixed to a point in the surface of a smooth horizontal cylinder is passed below the crlinder and carried round over the top, the other end being allowed to hang freely. Shew that unless the portion which hangs vertically be longer than the diameter of the cylinder, the cord will slip off, so as to hang down from the fixed point without passing below the cylinder.

14. If a uniform string hang in the form of a parabola by the action of normal forces only, the force at any point $P$ varies as $(S P)^{-1}, S$ being the focus.

15. If a string without weight touch a given cylinder in fth part of its circumference and in a plane perpendicular to its axis, what tension at one extremity will support a weight of $100 \mathrm{lbs}$. suspended at the other, friction being supposed to be thth part of the pressure? To what will this tension be reluced if the string is wound round 1 th circumferences?

16. If $\mu=f$, and a string without weight passes twice round a post, prove, by taking approximate ralues of $e$ and $\pi$. that any furce will support another more than twenty times as great.

17. If two scales, one enntaining a weight $P$ and the other a weight $Q$, be suspended by a string without weight 
over a rough sphere, and if $Q$ be on the point of descending, then the weight $\frac{Q^{2}-P^{n}}{P}$ put into the opposite scale will make that scale be on the point of descending.

18. Two equal weights $P, P^{\prime}$ are connected by a string without weight which passes over a rough fixed horizontal cylinder; compare the forces required to raise $P$ according as $P^{\prime}$ is pushed up or $P^{\prime}$ pulled down.

19. $A, B, C$ are three rough pegs in a vertical plane: $P, Q, R$ are the greatest weights which can be severally supported by a weight $W$, when connected with it by strings without weight passing over $A, B, C$, over $A, B$, and over $B, C$ respectively; shew that the coefficient of friction at $B$ is $\frac{1}{\pi} \log \frac{Q \cdot R}{P \cdot W}$.

20. A light thread, whose length is $7 a$, has its extremities fastened to those of a uniform heavy rod whose length is $5 a$, and when the thread is passed over a thin round peg, it is found that the rod will hang at rest, provided the point of support be anywhere within a space $a$ in the middle of the thread; determine the coefficient of friction between the thread and the peg when the rod hangs in a position bordering on motion, and find its inclination to the horizon and the tensions of the different parts of the string.

Results. The coefficient of friction is determined by the equation $e^{\mu / m}=\frac{4}{3}$. The inclination of the rod to the horizon is $\cos ^{-1} \frac{24}{25}$.

21. From a fixed point a heavy uniform chain hangs down so that part of the chain rests on a rough horizontal plane; find the least length of chain that may be in contact with the plane.

22. A heavy chain of weight $W$ rests entirely in contact with the arc of a rough closed vertical curve in a state bordering on motion. If $\tan \alpha$ be the coefficient of friction, shew that the resultant normal pressure on the circle is equal to $W \cos \alpha$, and that its direction makes an angle $\alpha$ with the vertical. 
23. A beavg chain of tengeth $l$ reats partly on a rusegh horizontal table and the remainder pasing over the smoeth edge of the table, (which is roumled off into the furm of a semicyliniler of radius a) hangs freely down; shew that if a be the least length on the table consistent with equilibrium,

$$
=(a+1)=l-\frac{1}{2} \pi a+a \text {. }
$$

24. A heary uniform chain is hung round the circumference of a rough vertical circle of given radius. How much lower muat one end of the chain hang than the other when it is on the point of motion?

Result. Ift $a$ be the leneth of the longer piece which hangs diwn, $b$ the length of the shorter piece, $r$ the radius of the circle, $\tan \beta$ the coefficient of friction; then

$$
\cos \theta=\frac{a-r \sin 2 \beta}{b+r \sin 2 \beta} \text {. }
$$

25. A uniform beam of weight $W$ is moveable alont a hinge at one extremity, and has the other attached to a string without weight which, passing over a very small rough pog placed vertically above the hinge, and at a distance from it equal to the length of the beam, supports a weight $P$ : shew that if $\theta$ the the inclination of the beam to the vertical when it is just on the point of falling, then

$$
W \sin \frac{1}{g} \theta=P e^{\frac{\operatorname{sic}+\theta}{\delta}}
$$

Find also the strain on the hinge.

26. One end of a heavy chain is attached to a fixed point $A$, and the other end to a weight which is placed on a rough horizontal plane passing through $A$, and the chain hangs through a slit in the horizontal plane. Shew that if I be the length of the chain, $a$ the greatest distance of the weight from $I_{\text {at }}$ which equilibrium is possibte, $\mu$ the cosfficient of friction, and $n$ twice the ratio of the given weight to the weight of the chain,

$$
\mu(1+n) e^{\sqrt{n+i}(i+n)}=1+\sqrt{ }\left(1+\mu^{2}(1+n)^{n}\right\} \text {. }
$$


27. A uniform string acted on by a central force assumes the form of an arc of a circle; determine the law of the force, the centre of force being on the circumference of the circle.

Result. The force varies inversely as the cube of the distanca.

28. A smooth sphere rests upon a string without weight fastened at its extremities to two fixed points; shew that if the arc of contact of the string and sphere be not less than $2 \tan ^{-1} \frac{48}{65}$, the sphere may be divided into two equal portions by means of a vertical plane without disturbing the equilibrium.

29. Shew that if a chain exactly surrounds a smooth vertical circle, so as to be in contact at the lowest point without pressing, the whole pressure on the circle is double the weight of the chain, and the tension at the highest point is three times that at the lowest.

30. Two strings without weight of the same length have each of their ends fixed at each of two points in the same horizontal plane. A smooth sphere of radius $r$ and weight $W$ is supported upon them at the same distance from each of the given points. If the plane in which each string lies makes an angle $\alpha$ with the horizon, prove that the tension of each is $\frac{W a}{8 r} \operatorname{cosec} \alpha ; a$ being the distance between the points.

31. A uniform heavy chain hangs over two smooth pegs at a distance $2 a$ apart in the same horizontal plane. When there is equilibrium, $2 s$ is the length of the chain between the pegs, which hangs in the form of a catenary, $c$ is the length of a portion of the chain whose weight is equal to the tension at the lowest point, and $h$ the length of the end that hangs down vertically. If $\delta s$ and $\delta h$ be the small increments of $s$ and $h$ corresponding to a small uniform expansion of the chain, shew that $\delta s: \delta h=s . c-h . a: h . c-s . a$.

32. A uniform heavy chain is placed on a rough inclined plane; what length of chain must hang over the top of the plane, in order that the chain may be on the point of slipping up the plane? 
33. A uniform rod of length 6 has its encls attachod to the ends of a flexible string without weight of lengh a; this string is pasand over a very stuall eylimdrical pes, and when the rod hangs in its limiting position of equilibrium, the parts of the string on ofpesite siles of the per are inclined to each other at an angle $a$. Shew that the oocticient of frietion between the string and peg is

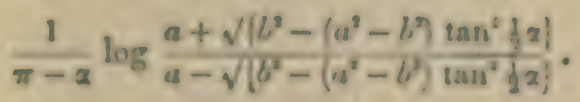

34. $A B, A C$ are two equal and uniform rods movenble about a fised hinge at $A, C B$ a uniform chain, equal in length to $A B$ or $A C$ and $\left(\frac{1}{n}\right)^{\text {th }}$ of its weight, cornects the encls $B$ and $C$; shew that in the position of equilibium, the angle $\theta$ which either rod makes with the horizon is giren approximately by the equation

$$
\cos \theta=\frac{1}{3(n+1)^{n}}
$$

$n$ being large compared with unify.

35. A heavy uniform beam has its extremities attached to a string which passes round the are of a rough vertical circle; if in the limiting position of equilibrium the beam be inclined at an angle of $60^{\circ}$ to the vertical, and the portion of string in contact with the circle eorer an are of $270^{\circ}$, shew that the coefficient of friction is $\frac{1}{3 \pi} \log 3$.

36. A uniform string just circumscribes a given smooth circle, and is attracted by a furce varying as the distance to a point within the circle. Find the tension at any joint, supposing it to ranish at the point neareat to the centre of force, and shew that the force at the greatest distance

$$
=\frac{\text { whole pressure on circle }}{\text { mass of the string }} \text {. }
$$


37. A heavy string whose length is $\frac{\pi}{2} a$ rests on the circumference of a rough vertical circle of radius $a$; if the string be in a position of limiting equilibrium, and if $\beta$ be the angular distance of its highest extremity from the vertex of the circle, shew that

$$
\tan \beta=\frac{\left(1-\mu^{2}\right) e^{\frac{\mu \pi}{3}}-2 \mu}{1-\mu^{2}+2 \mu e^{\frac{\mu \pi}{3}}},
$$

and explain this result when $\left(1-\mu^{2}\right) e^{\mu \pi}-2 \mu$ is negative.

Also if $\mu$ be such that $\beta=0$, shew that the whole pressure on the curve is to the weight of the string as 2 is to $\pi \mu$. 


\section{(255)}

\section{CHAPTER XII.}

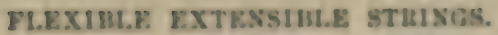

195. Is the proceding Chapter we considered the equilitrium of flexible incxtensible strings; we now proced to some propositions relative to flexible extensible strings. Such strings are also called elastic strings.

When a uniform extensible string is stretehed by a force, it is foumd by experiment that the extension varies as the product of the orignal length and the stretching force. Thus if $T$ represent the furce, $l$ the original length, $l$ the stretehed length,

$$
l-l^{\prime}=\frac{l T}{\lambda},
$$

where $\lambda$ is some constant depending on the nature of the string.

The fact expressed by this equation is called Hooke's law, from the name of its disooverer.

The quantity $\lambda$ is sometimes called the modulus of elasticity.

In the equation $l-l=\frac{r T}{\lambda}$ if we put $T=\lambda$ we obtain $l=2 l$; thus the modulus of elasticity for any uniform elastic string is equal to the tension reqguired to stretch that string to double its natural length.

109. An clastic string hins a weriglte attached to one end, it is fisstened at the other and hangs vertically: determine the extension of the string, taking its ouen wecight into account. 
Let $A^{\prime} B^{\prime}$ represent the natural length of the string; $A B$ the stretched length. Let $A^{\prime} P^{\prime \prime}=x^{\prime}, P^{\prime} Q^{\prime}=\delta x^{\prime}$. Suppose $A^{\prime} P^{\prime}$ stretched into $A P$, and $P^{\prime} Q^{\prime}$ into $P Q$; let $A P=x, P Q=\delta x$. Let $A^{\prime} I B^{\prime}=a^{\prime}$, $w=$ the weight of the string, and $W$ be the attached weight.

Let $T$ be the tension at $P$, and $T+\delta T$ the tension at $Q$. Then the element $P Q$ is acted on by the forces $T$ and $T+\delta T$ at its ends, and by its own weight; its weight is the same as that of $P^{\prime} Q$, that is $\frac{\delta x^{\prime}}{a^{\prime}} w$;

therefore

$$
T+\delta T-T+\frac{\delta x^{\prime}}{a^{\prime}} w=0,
$$

or

$$
\frac{d T}{d x^{\prime}}=-\frac{w}{a^{\prime}} \text { ultimately }
$$

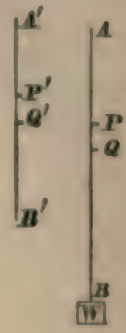

therefore

$$
T=-\frac{w x^{\prime}}{a^{\prime}}+\text { constant. }
$$

The value of the constant must be found by observing that when $x^{\prime}=a^{\prime}, T=W$; therefore

$$
W=-w+\text { constant ; }
$$

therefore

$$
T=W+w\left(1-\frac{x^{\prime}}{a^{\prime}}\right) \text {. }
$$

Also the elcment $P Q$ may be considered ultimately uniform and stretched by a tension $T$; hence, by the experimental law,

$$
\delta x=\delta x^{\prime}\left(1+\frac{T}{\lambda}\right)
$$

therefore

$$
\begin{aligned}
\frac{d x}{d x^{\prime}} & =1+\frac{T}{\lambda}, \\
& =1+\frac{W}{\lambda}+\frac{w}{\lambda}\left(1-\frac{x^{\prime}}{a^{\prime}}\right) .
\end{aligned}
$$

Integrate; thus

$$
x=x^{\prime}\left(1+\frac{W+w}{\lambda}\right)-\frac{v x^{\prime 2}}{2 \lambda a^{\prime}} .
$$

No constant is required because $x=0$ when $x^{\prime}=0$. 
Let a denote the stretched length of the string; then pusting $x^{\prime}=a^{\prime}$, we have

$$
a=a^{\prime}\left(1+\frac{W+\infty}{\lambda}\right)-\frac{m a^{\prime}}{2 \lambda}=a^{\prime}\left(1+\frac{W+1}{\lambda}\right) .
$$

Thus the extension is the snme as would be produced if an elartic string of length $a$, the weight of which might be neglected, were stretehed by a weight $W+$ dre at its end.

200. In the solntion of the preceding problem we might have arrived at equation (2) by observing that the tension at any point mast be equal to the weight of the string lelow that point together with $W_{\text {; }}$ but the method we adopted is more uartul a a gruide to the solution of similar problems. It is perhaps not superflnous to notioe an error into which stadents often fall; since the element $\delta x$ is acted on by a tension $T$ at one end, and $T+\delta T$ or ultimately $T$ at the other end, $2 T$ is considered the stretching fores, and instend of (3)

$$
8 x-8 x^{\circ}\left(1+\frac{2 T}{\lambda}\right)
$$

is nsed. This would be of no consequence if uniformly adopted, for it would only amount to using $\frac{1}{2} \lambda$ instead of $\lambda$ in (3); but mistakes arise from not adhering to one system or the other. It should be observed that if a string without weight be acted on by a foree $T$ at ench end, it is in the same state of tension as if it were fustened at one end and acted on by a force $T$ at the other.

201. The equations of Art. 187, and Art. 196 may be npplied to an elastic string in equilibrium. They may also be modified as follows, if we wish to introdnce the unstretched length of the string instead of the stretched length.

Let $\delta$ and $\delta s^{\prime}$ represent the natural lengths which become $s$ and $\delta$ s by stretching; let $m^{\prime} \delta d^{\prime}$ be the mass of an element before stretching, and mos the mass of the same element after stretching; then

$$
\begin{aligned}
& m \delta s=v^{\prime} \delta s^{\prime}, \\
& \delta s=\delta \delta^{\prime}\left(1+\frac{T}{\lambda}\right) ;
\end{aligned}
$$


therefore

$$
m\left(1+\frac{T}{\lambda}\right)=m^{\prime}
$$

Hence the first equation of equilibrium of Art. 187 may be written

$$
\frac{d}{d s}\left(T \frac{d x}{d s}\right)+\frac{m^{\prime} X}{1+\frac{T}{\lambda}}=0 ;
$$

and the other two equations may be written similarly.

Equation (2) of Art. 188, or equation (4) of Art. 196 becomes

$$
\begin{aligned}
& \left(1+\frac{T}{\lambda}\right) \frac{d T}{d s}+m^{\prime}\left(X \frac{d x}{d s}+Y \frac{d y}{d s}+Z \frac{d z}{d s}\right)=0 ; \text { therefore } \\
& \lambda\left(1+\frac{T}{\lambda}\right)^{2}+2 m^{\prime} \int\left(X \frac{d x}{d s}+Y \frac{d y}{d s}+Z \frac{d z}{d s}\right) d s=\text { constant, }
\end{aligned}
$$

provided $m^{\prime}$ be constant; that is, provided the string in its unstretched state be uniform.

Since $\left(1+\frac{T}{\lambda}\right)^{2}=\left(\frac{d s}{d s}\right)^{2}$, the last equation may be used to connect $s$ and $s^{\prime}$, and thus find the extension of the string.

202. We may apply the preceding $\Lambda$ rticle to the case in which the weight of the string is the only force acting on it, the string being supposed originally uniform, and fixed at two points.

In this case $X=0, Y=-g, Z=0$, as in Art. 190; therefore

$$
\begin{array}{r}
\frac{d}{d s}\left(T \frac{d x}{d s}\right)=0 \ldots \ldots \ldots \ldots \ldots \ldots \ldots(1) . \\
\left(1+\frac{T}{\lambda}\right) \frac{d}{d s}\left(T \frac{d y}{d s}\right)-m^{\prime} g=0 \ldots \ldots \ldots \ldots \ldots(2) .
\end{array}
$$

From (1) $T \frac{d x}{d s}=$ a constant $=m^{\prime} c g$ suppose;

therefore

$$
T=m^{\prime} \operatorname{cg} \sec \psi
$$

where $\psi$ is the angle which the tangent to the curve at the point $(x, y)$ makes with the axis of $x$. 
Hence (2) gives

therefore

$$
\left(1+\frac{\operatorname{mic} g}{\lambda} \sec \psi\right) \frac{d \tan \psi}{d s}=\frac{1}{c} \ldots \ldots \ldots . .(0)
$$$$
\left(1+\frac{m i g}{\lambda} \sec \psi\right) \frac{d \tan \psi}{d x} \frac{d x}{d s}=\frac{1}{c} \text {, }
$$

thus

$$
\cos \psi\left(1+\frac{\operatorname{mic}^{\prime} \sec \psi}{\lambda}\right) \frac{d \tan \psi}{d x}=\frac{1}{0} \text {, }
$$

that is

$$
\frac{1}{\cos \psi} \frac{d \psi}{d x}+\frac{\operatorname{mig}}{\lambda} \frac{d \tan \psi}{d x}=\frac{1}{c} \text {; }
$$

therefore, by integration,

$$
\int \frac{1}{\cos \psi} \frac{d \psi}{d x} d x+\frac{m^{\prime} \operatorname{cg}}{\lambda} \tan \psi=\frac{x}{c} ;
$$

and

$$
\int \frac{1}{\cos \psi} \frac{d \psi}{d x} d x=\int \frac{d \psi}{\cos \psi}=\log \frac{1+\sin \psi}{\cos \psi} ; \text { thus }
$$

$$
\log \frac{1+\sin \psi}{\cos \psi}+\frac{\operatorname{ni}^{\prime} c g}{\lambda} \tan \psi=\frac{x}{c}
$$

No constant is required in the integration if we suppose the axis of $y$ to pass through the lowest point of the eurve, for there $\psi=0$.

From (4) we may deduce

$$
\sin \psi\left(1+\frac{m^{\prime} c y}{\lambda} \sec \psi\right) \frac{d \tan \psi}{d y}=\frac{1}{c}
$$

therefore, by integration,

$$
\sec \psi+\frac{m^{\prime} c}{2 \lambda} \tan ^{2} \psi=\frac{y}{c} .
$$

No constant is required in the integrntion if we suppose the origin of co-ordinates to be at the distance $c$ below the lowest point of the curve. 
From equation ( 7 ) we may find sec $\psi$ in terms of $y$, and then $\cos \psi$ and $\sin \psi$ can also be found; thus by substituting in (5) we could obtain the equation between $x$ and $y$ : this equation however would be very complex.

In a particular case we may easily obtain an approximate value of $y$ in terms of $x$. Let $\lambda=m^{\prime} g l$; then (5) may be written

therefore

$$
\begin{aligned}
& \frac{1+\sin \psi}{\cos \psi}=e^{\frac{x}{\sigma}-\frac{e}{i} \tan \psi}, \\
& \frac{\cos \psi}{1+\sin \psi}=e^{-\frac{\pi}{e}+\frac{e}{i} \tan \psi}
\end{aligned}
$$

therefore by addition and reduction

therefore

$$
\frac{2}{\cos \psi}=e^{\frac{x}{6}-i \tan \psi}+e^{-\frac{\pi}{6}+i \tan \psi},
$$

Now suppose $\frac{c}{l}$ is a very small quantity, put $u$ for $\frac{1}{2}\left(e^{\frac{x}{c}}-e^{-\frac{x}{c}}\right)$ and $v$ for $\frac{1}{2}\left(e^{\frac{x}{e}}+e^{-\frac{x}{b}}\right)$; then the last equation gives

$$
\tan \psi=u-\frac{c v}{l} \tan \psi+\frac{c^{2} u}{1.2 l^{2}} \tan ^{2} \psi-\frac{c^{3} v}{\left[3 l^{3}\right.} \tan ^{3} \psi+\ldots ;
$$

from this we can find $\tan \psi$ approximately, and then $\sec \psi$ will be known approximately, and by substituting in (7) we shall obtain approximately $y$ in terms of $x$.

Equation (2) may also be written

$$
\frac{d}{d s^{\prime}}\left(T \frac{d y}{d s}\right)=m^{\prime} g
$$

therefore

$$
\frac{d}{d s^{\prime}}\left(m^{\prime} c g \frac{d y}{d x}\right)=m^{\prime} g ;
$$

therefore, by integration,

$$
\frac{d y}{d x}=\frac{s^{\prime}}{c}
$$


here $g^{\prime}$ denotes the natural length of that portion of the string which is between the lowest point and the point $(x, y)$.

Hence for tan $\psi$ in (3) and (7) we may put $\frac{\varepsilon^{\prime}}{c}$, and make corresponding substitutions for $\sin \psi$ and $\cos \psi$. Thus (7) becomes

$$
\sqrt{ }\left(\theta^{0}+\theta^{\prime \prime}\right)+\frac{m^{\prime} g s^{n}}{2 \lambda}=y \ldots \ldots \ldots \ldots \ldots \ldots(8) .
$$

As an example of these furmula suppose that a heavy unifurn elastie string hangs in equilitirium over two monoth pegs in a herizontal plane, and let it be reguired to find the depth of the ends of the string below the vertex of the curved portion.

From (3) the tension at any point of the curve is

$$
\text { - } m^{\circ} g \sqrt{ }\left(\sigma^{2}+s^{2}\right) \text {. }
$$

Iet $\tau$ be the natural length of the portion which hangs over one of the pegs; then the wcight of this portion is migt. Iet $s$ denote the unstretched length of the portion between the vertex and one feg ; then by equating the two expressions for the tension, we have

$$
m^{\prime} g l^{\prime}=m^{\prime} g \sqrt{ }\left(\sigma^{2}+s^{\prime 2}\right),
$$

therefore

$$
l=\sqrt{ }\left(c^{3}+s^{\prime 3}\right)
$$

thus from (8) and (9)

$$
\boldsymbol{l}+\frac{m^{\prime} g\left(l^{2}-c^{2}\right)}{2 \lambda}=y \ldots \ldots \ldots \ldots(10) .
$$

Suppinse $l$ to be the length to which a string of natural length $\zeta$ hanging vertically would be stretched; then by Art. 199,

$$
l=l\left(1+\frac{m^{\prime} g l}{2 \lambda}\right)
$$

By (10) and (11)

$$
l=y+\frac{m i y c^{3}}{2 \lambda} \text {. }
$$


Thus the end of the string descends to the depth $\frac{m^{\prime} g c^{2}}{2 \lambda}$ below the axis of $x$, and therefore to the depth $c\left(1+\frac{m^{\prime} c g}{2 \lambda}\right)$ below the vertex of the curve.

\section{EXAMPLES.}

1. Two equal heavy beams, $A B, C D$, are connected diagonally by similar and equal elastic strings $A D, B C$ : shew that if the natural length of each string equals $A B$, and the elasticity be such that the weight of $A B$ would stretch the string to three times its natural length, then

$$
\frac{1}{A B}=\frac{1}{B C}+\frac{1}{A C} \text {. }
$$

2. An elastic string will just reach round two pegs in a horizontal plane; a ring whose weight would double the length of the string hanging from a point is slung on it: shew that if $\theta$ be the inclination of two portions of the string to the horizon,

$$
\sin 2 \theta=2(\sqrt{ } 2-1) .
$$

3. An elastic string has its ends attached to those of a uniform beam of the same length as the unstretched string, the weight of the beam being such as would stretch the string to twice its natural length; shew that when the system is hung up by means of the string on a smooth peg, the inclination $\theta$ of the string to the vertical will be given by the equation

$$
\tan \theta+2 \sin \theta-2=0 .
$$

4. Three equal circular dises are placed in contact in a vertical plane with their centres in the same horizontal line, and an endless elastic cord wound alternately above and below them, so as to touch every point of their circumferences without being stretched beyond its natural length. When the support of the middle disc is removed, the centres of the 
three form a right-angled triangle. Shew that the modulus of elasticity of the cord is $\frac{I V}{2} \frac{3 \pi}{4-\pi}, W$ being the weight of the disc.

5. $\Lambda$ fine elastic string is tied round two equal cylinders whos surfaces are in contact and axes parallel, the string not being stretched beyond its natural length; one of thie cylinders is furned thrugh two right angles, so that the axes are again parallel: find the tinsion of the string, supposing a weight of $1 \mathrm{lb}$. would stretch it to twice its natural length.

$$
\text { Resule. } \frac{\pi-2}{\pi+2} \text { of a lb. }
$$

6. Two equal and similar elastic strings $A C, B C$, fixed at two points $A, B$ in the same horizontal line, support a given weight at $C$. The extensibility and original lengths of the strings being given, find an equation for determining the angle at which each string is inclined to the horizon, and dednce an approximate value of the angle when the extensibility is very small.

7. Six equal rods are fastened together by linges at each end, and one of the rods being supported in a horizontal position the opposite one is fastened to it by an clastie string joining their mialle points. Supposing the modulus of elasticity is equal to the weight of each rol, find the original length of the string in order that the hexagon may be equiangular in its position of equilibrium.

$$
\text { Result. } \frac{a \sqrt{ } 3}{4} \text {, where } a \text { is the length of a rod. }
$$

8. An unstrefched elastic string without weight has $n$ equal weights attached to it at equal distances, and is then suspended from one end. Prove that the inerease of length is half what it would be if the same string were stretehed by a weight equal to $n+1$ of the former hanging at one end.

9. Three equal cylindrical rods are placed symmetrically round a fourth of the same rading, and the bundle is then - surrounded by two equal elastic bands at eqqual distances 
from the two ends; if each band when unstretched would just pass round one rod, and a weight of $1 \mathrm{lb}$. would just stretch it to twice its natural length, shew that it would require a force of $9 \mathrm{lbs}$. to extract the middle rod, the coefficient of friction being equal to $\frac{1}{6} \pi$.

10. Two elastic strings are just long enough to fit on a sphere without stretching; they are placed in two planes at right angles to each other, and the sphere is suspended at their point of intersection. If $2 \theta$ be the angle subtended at the centre by the are which is unwrapped, shew that

\section{$\theta$ being supposed small.}

$$
\theta^{4}=\frac{3 \pi}{4} \frac{W}{\lambda},
$$

11. In the common catenary, if the string be slightly extensible, shew that its whole extension will be proportional to the product of its length and the height of its centre of gravity above the directrix.

12. A uniform rough cylinder is supported with its axis horizontal by an elastic string without weight; the string lies in the plane which is perpendicular to the axis of the cylinder, and passes through its centre of gravity; the ends of the string are attached to points which are in the same horizontal plane above the cylinder and at a distance equal to the diameter of the cylinder. Find how much the string is stretched.

Result. Let $2 \mathrm{~W}$ be the weight of the cylinder, $a$ the radius of the cylinder, $b^{\prime}$ the natural length of each vertical portion of the string; then the extension is

$$
\frac{2 l^{\prime} W}{\lambda}+\frac{2 a}{\mu} \log \frac{\lambda+W e^{\frac{\mu \pi}{2}}}{\lambda+W} .
$$

13. A heavy string very slightly elastic is suspended from two points in the same horizontal plane; shew that if $c, l$ be the lengths of unstretched string whose weights are respectively equal to the tension at the lowest point and the modulus of elasticity, the equation to the catenary will be very approximately

$$
y=\frac{c}{2}\left\{e^{\frac{2}{e}}+e^{-\frac{z}{c}}-\frac{c}{4 l}\left(e^{\frac{\pi}{e}}-e^{-\frac{\pi}{e}}\right)^{2}\right\} .
$$


14. A weight $P$ just supports another weight $Q$ by means of a fine elastic string passing over a rough cylimler whose axis is horizontal. If $\lambda$ be the modulus of elasticity, $\mu$ the coefficient of friction, and $a$ the radius of the cylinder, shew that the extension of that part of the string which is in contact with the cylinder is

$$
\frac{a}{\mu} \log \frac{Q+\lambda}{\mu+\lambda} .
$$

15. A sphere placed on a horizontal plane is dirided by a vertical plane into two equal parta, which are just held together by an elastic string, which passes round the greatest horizontal section; find the original length of the string.

$$
\text { Rearele. } \frac{32 \lambda \pi u}{16 \lambda+3 W} \text {. }
$$

16. Four equal heavy rods are fastened to one another by hinges so is to form a square $A B C D ; A$ and $C$ are connected by an clastic string whose natural length is equal to the diagunal $A C$, and the system is suspended from the point $A$; find the position of equilibrium.

Resule. Let $W$ be the weight of a rod, $\theta$ the inclination of each rod to the vertical; then

$$
\cos \theta=\frac{1}{\sqrt{ } 2}\left(1+\frac{2 W}{\lambda}\right) \text {. }
$$

17. An elastic band, whose unstretched length is $2 a$, is placed round four rough pegs $A, B, C, D$, which constitute the angular points of a square whose side is $a$; if it be taken hold of at a point $P$, between $A$ and $B$, and pulled in the direction $A B$, shew that it will begin to slip round $A$ and $B$ at the same time if

$$
A P=\frac{a}{1+e^{5}} .
$$

19. An elastic string without weight of variable thickness is extended by a given force; find the whole extension.

19. An clastic string whose density varies as the distance from one end, is suspended by that end and stretehed by its 
own weight. If $W$ be the weight of the string, $l^{\prime}$ its unstretched length, $l$ its stretched length, shew that

$$
l=l^{\prime}\left(1+\frac{2 W}{3 \lambda}\right) \text {. }
$$

20. A circular elastic string is placed on a smooth splicre so that the whole string is in one horizontal plane; the string subtends when unstretched an angle $2 \alpha$ at the centre, and an angle $2 \theta$ when in a position of equilibrium; shew that

$$
\sin \theta=\sin \alpha\left(1+\frac{a}{c} \sin \alpha \tan \theta\right),
$$

where $a=$ radius of sphere, and $c$ depends on the nature of the string.

21. A heary uniform elastic string rests horizontally on a portion of a surface of revolution, of which the axis is vertical, in every position: prove that the generating curve is a parabola a diameter of which is the axis of revolution.

22. A heavy elastic string surrounds a smooth horizontal cylinder, so that the surface of the cylinder is subject to no pressure at the lowest point; find the pressure at any point of the cylinder, and the tension of the string; its modulus of elasticity being equal to the weight of a portion of string the natural length of which is $\frac{8}{8}$ of the diameter of the cylinder.

23. A uniform heavy elastic string, whose natural length is $a$, is stretched and placed in equilibrium on a rough inclined plane; find the tension at any point, and shew that the direction of the friction changes at a point of the string, the natural distance of which from the upper end is

$$
\frac{a}{2}\left(1+\frac{\tan \alpha}{\mu}\right),
$$

where $a$ is the inclination of the plane to the horizon.

24. A heavy elastic cord is passed through a number of fixed smooth rings. Shew that in the position of equi- 
librium its extremities will lie in the anme horizontal plane. The same will also be the case if the cord rest upon any smooth surface.

25. An elnatie string is laid on a cycloidal arc, the plane of which is vertical and vertes upwarls, and when atreuteol by its own wcight is in contact with the whole of the cycloind; the modulns of elnaticity is the weicht of a portion of the string whose natural length is twice the diameter of the generating circle; find the natural length of the string.

Resule. It is equal to the circumference of the generating circle. 


\title{
CHAP'TER XIII.
}

\author{
ATTRACTIONS.
}

203. IT appears from considerations which are detailed in works on Physical Astronomy, that two particles of matter placed at any sensible distance apart attract each other with a force directly proportional to the product of their masses, and inversely proportional to the square of their distance.

Suppose then a particle to be attracted by all the particles of a body; if we resolve the attraction of each particle of the body into components parallel to fixed rectangular axes, and take the sum of the components which act in a given direction, we obtain the resolved attraction of the whole body on the particle in that direction, and can thus ascertain the resultant attraction of the body in magnitude and direction. We shall give some particular examples, and then proceed to general formulæ.

204. To find the attraction of a uniform straight line on an external point.

By a straight line we understand a cylinder such that the section perpendicular to its axis is a curve, every chord of which is indefinitely small.

Let $A B$ be the line, $P$ the attracted particle; take $A$ for the origin, and $A B$ for the direction of the axis of $x$. Draw $P L$ perpendicular to $A x$; let $A B=l$, $A L=a, P L=b$. Let $M$ and $N$ be adjacent points in the line, $A M=x$, $M N=\delta x$. If $\rho$ be the

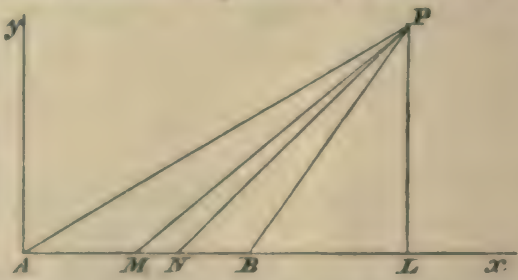
density of the line, and $\boldsymbol{\kappa}$ the area of a section perpendicular 
to its length, the mass of the element is prôx. Jet $m$ be the mass of $P$; then the attraction of the element $M . N$ on $P$ is (Art. 203)

$$
\frac{\mu m \rho x \delta x}{(1, M)^{x}}
$$

where $\mu$ is some constant quantity. Hence, the resolved part of the attraction of the element parallel to the axis of $x$, is

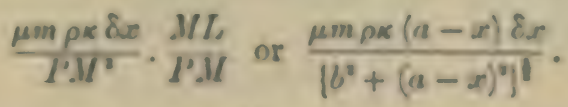

Also the resolval part of the attraction of the element paralled to the axis of $y_{1}$ is

$$
\frac{\mu m \rho \alpha \delta x}{P M I^{2}} \cdot \frac{P L}{P M} \text { or } \frac{\mu m \rho x b \delta r}{\left[b^{2}+(a-x)^{2}\right]^{\circ}} \text {. }
$$

Iat $X$ and $Y$ be the resolved parts of the attraction of the line, parallel to the axes of $x$ and $y$ respectively; then

$$
\begin{aligned}
& X=\mu m \rho x \int_{0}^{l} \frac{(a-x) d x}{\left\{b^{3}+(a-x)^{2}\right\}}, \\
& Y=\mu m \rho x \int_{0}^{l} \frac{b d x}{\left\{b^{2}+(a-x)\right\}^{3}} .
\end{aligned}
$$

Now

$$
\int \frac{(a-x) d x}{\left[b^{3}+(a-x)^{2}\right\}}=\frac{1}{\left\{b^{2}+(a-x)^{2}\right]^{3}} \text {; }
$$

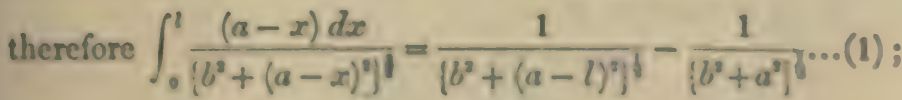

$$
\int \frac{d x}{\left[b^{3}+(a-x)^{2}\right]^{2}}=-\frac{a-x}{b^{2}\left[b^{3}+(a-x)^{2}\right]^{7}} ;
$$

therefore

$$
\int_{0}^{l} \frac{b d x}{\left\{b^{2}+(a-x)^{2}\right\}^{1}}=\frac{1}{b}\left[\frac{a}{\left(b^{2}+a^{2}\right)^{1}}-\frac{a-l}{\left[b^{2}+(a-l)^{2}\right)^{4}}\right] \ldots . .(2)
$$


Hence, putting $f$ for $\mu \rho \kappa$, we have

$$
\begin{aligned}
& X=f m\left\{\frac{1}{P B}-\frac{1}{P A}\right\} \ldots \ldots \ldots \ldots \ldots \ldots(3), \\
& Y=\frac{f m}{P L}\left\{\frac{A L}{P A}-\frac{B L}{P B}\right\} \ldots \ldots \ldots \ldots \ldots \ldots(4) .
\end{aligned}
$$

Let $A P L=\alpha, B P L=\beta, A P B=\gamma$; then

$$
\begin{aligned}
& X=\frac{f m}{P L}(\cos \beta-\cos \alpha), \\
& Y=\frac{f m}{P L}(\sin \alpha-\sin \beta) ;
\end{aligned}
$$

therefore $\sqrt{ }\left(X^{2}+Y^{2}\right)=\frac{f m}{P L} \sqrt{ }\left\{(\cos \beta-\cos \alpha)^{2}+(\sin \alpha-\sin \beta)^{2}\right\}$

$$
=\frac{f m}{P L} \sqrt{ }(2-2 \cos \gamma)=\frac{2 f m}{H^{\prime} L} \sin \frac{1}{2} \gamma \ldots(5) .
$$

This gives the magnitude of the resultant attraction. Also

$$
\frac{X}{Y}=\frac{\cos \beta-\cos \alpha}{\sin \alpha-\sin \beta}=\tan \frac{\alpha+\beta}{2}
$$

'This shews that the direction of the resultant attraction bisects the angle $\triangle P B$.

If $L$ fall between $A$ and $B$, it will be seen from (1) and (2) that the expression for $X$ in (3) remains unchanged, but that for $Y$ in (4) is changed to

$$
\frac{f m}{P L}\left\{\frac{A L}{P A}+\frac{B L}{P B}\right\} \text {. }
$$

This will not affect the result in (5), and the direction of the resultant will still bisect the angle $A I^{\prime} B$.

From the investigation it appears that $X$ is the resolved attraction parallel to the axis of $x$ directed towards the axis of $y$, and $Y$ the resolved attraction parallel to the axis of $y$ and towards the axis of $x$. 
205. In the sbove investigation we have taken $m$ to denote the mass of the attracted particle; in future we shall always suppose the mass of the attracted pariele to be denoted by unity. In order to furm a precien idea of the quantity $\mu$, we may suppose two particles each having its mass equal to the unit of mass, then $\mu$ will be the whole force which one of these exerts on the other when the distance between them is the unit of length. As, however, by properly cluoceing the unit of mass we may make $\mu=1$, we shall not in fature consider it necessary to introtuce $\mu$.

206. To find the attruction of a circular are en a partiele situated at the centre of the circle.

Let $A B$ be any circular are; thruagh $O$ the centre of the circle draw a line hisecting the angle $A O B$, and take this line for the axis of $x$. Iat $P O x=\theta, Q Q P=\delta \theta$, $A O B=2 x, O B=r$. 'The attraction of the element $I^{\prime} Q$ resolved paraliel to the axes of $x$ and $y$ respectively is, if $\rho$ and $k$ have the same meaning as in Art. 204,

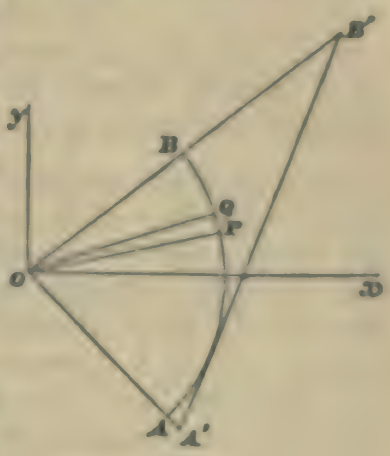

$$
\frac{k \rho r \varepsilon \theta}{r^{2}} \cos \theta \text { and } \frac{k \rho r \delta \theta}{r^{2}} \sin \theta \text {; }
$$

therefure

$$
\begin{aligned}
& X=\frac{\kappa \rho}{r} \int_{-\infty}^{+\infty} \cos \theta d \theta=\frac{2 \kappa \rho}{r} \sin \pi, \\
& I=\frac{\kappa \rho}{r} \int_{-\infty}^{+\infty} \sin \theta d \theta=0 .
\end{aligned}
$$

By comparing these resules with those in Art. 204, it appears that the attraction of a circular arc on a particle at the centre is the same in magnitule and direction as that of any straight line $A B$ which touches the are $A B$ and is terminated by the lines $O A$ and $O B$ producod, the are and line being supposed to have the same density, and the areas of their tratisverse sections equal. 
If $O P$ and $O Q$ be produced to meet the line $A^{\prime} B^{\prime}$ in points $P^{\prime}$ and $Q^{\prime}$ respectively, it may be shewn that the attraction of the clement $P^{\prime} Q^{\prime}$ on a particle at $O$ is equal to that of $P Q$, and in this manner we might prove what we have just shewn, that the attractions of $A B$ and $A^{\prime} B^{\prime}$ on a particle at $O$ are equal and coincident. 'This proposition is given in Earnshaw's Dynamics, p. 826.

It casily follows, that if a particle be attracted by the three sides of a triangle, it will be in equilibrium if it be placed at the centre of the circle inscribed in the triangle.

207. To find the attraction of a uniform circular lamina on a particle situated in a straight line drawn through the centre of the lamina at right angles to its plane.

Suppose $C$ the centre of the circle $D A B$, the plane of the paper coinciding with one face of the lamina, and the attracted particle being in a straight line drawn through $C$ perpendicular to the lamina and at a distance $c$ from $C$. Describe from the centre $C$ two adjacent concentric circles, one with radius $C P^{\prime}=r$, and the other with radius $C Q=r+\delta r$. Let $\kappa$ denote the thickness of the lamina, which is supposed to be an in-

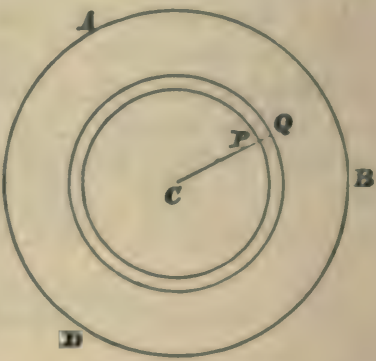
definitely small quantity, then the mass of the circular ring contained between the adjacent circles is $2 \pi \rho \kappa r \delta r$. Every particle in this circular ring is at a distance $\sqrt{ }\left(c^{2}+r^{2}\right)$ from the attracted particle; also the resultant attraction of the ring is in the straight line through $C$ at right angles to the lamina, and is equal to

$$
\frac{2 \pi \rho c r \delta r}{c^{2}+r^{2}} \cdot \frac{e}{\sqrt{\left(c^{3}+r^{4}\right)}},
$$

the factor $\frac{c}{\sqrt{\left(c^{2}+r^{2}\right)}}$ being the multiplier necessary in order to resolve the attraction of any element of the ring along the normal to the lamina through $C$. 
Ilesice, the resultant attraction of the whole lamina is

$$
2 \pi \rho a c \int_{0}^{b} \frac{r d r}{\left(\sigma^{2}+r^{2}\right)},
$$

where $b$ is the radius of the boundary of the lamina.

Now

$$
\int \frac{r d r}{\left(c^{2}+r^{2}\right)^{1}}=-\frac{1}{\sqrt{\left(e^{3}+r^{2}\right)^{2}}}
$$

therefore

$$
\int_{0}^{3} \frac{r d r}{\left(c^{3}+r^{5}\right)}=\frac{1}{c}-\frac{1}{\sqrt{\left(c^{3}+b^{5}\right)}}
$$

therefore the resultant attraction

$$
=2 \pi \rho x_{0}\left\{\frac{1}{0}-\frac{1}{\sqrt{\left(c^{5}+b^{5}\right)}}\right\} \text {. }
$$

If we suppose $b$ to become infinite, we obtain for the attraction of an infinite lamina on an external particle, the expression $2 \pi \rho x$, which is independent of the distance of the attracted particle from the lamina.

From the last result we can deduce the resultant attraction of a uniform plate of finite thickness, but of infinite extent, on an external particle. For, suppose the plate divided into an indefinituly large number of lamine, each of the thickness $x$; then the attraction of each lamina acts in a straight line through the attracted particle perpendicular to the surfaces of the plate, and is equal to $2 \pi \rho$ r. Hence, the resultant attraction will be found by adding the attractions of the lamina, and will be $2 \pi p h$, if $h$ be the thickness of the plate.

If a particle be placed on the exterior surfice of an infinite plate, the result just found will express the attraction of the plate on the particle. If it be placed in the interios of the flate at a distance $h$ from one of the bounding planes and $f$ from the other, the resultant attraction will be $2 \pi \rho\left(h^{\prime}-h\right)$ tovourds the latter plane.

208. By means of the preceding Article we can find the resultant attraction of a unifurm cylinder on a particle T. \&. 
situated on its axis. Suppose the cylinder divided into an indefinitely large number of lamina by planes perpendicular to its axis; let $x$ be the distance of a lamina from the attracted particle, $\delta x$ the thickness of the lamina, $b$ the radius of the cylinder; then the attraction of the lamina is

$$
2 \pi \rho\left\{1-\frac{x}{\sqrt{\left(x^{3}+b^{2}\right)}}\right\} \delta x \text {. }
$$

Suppose the attracted particle outside the cylinder at a distance $c$ from it; let $h$ be the height of the cylinder; then the resultant attraction of the cylinder

$$
\begin{aligned}
& =2 \pi \rho \int_{0}^{0+\lambda}\left\{1-\frac{x}{\sqrt{\left(x^{2}+b^{2}\right)}}\right\} d x \\
& =2 \pi \rho\left[h-\sqrt{ }\left\{(c+h)^{2}+b^{2}\right\}+\sqrt{ }\left(c^{2}+b^{2}\right)\right] .
\end{aligned}
$$

If we suppose $c=0$ so that the particle is on the surface of the cylinder the resultant attraction is

$$
2 \pi \rho\left\{h-\sqrt{ }\left(h^{2}+b^{2}\right)+b\right\} .
$$

209. To find the attraction of a uniform cone on a particle at its vertex, we begin with the expression

$$
2 \pi \rho\left\{1-\frac{x}{\sqrt{\left(x^{2}+b^{2}\right)}}\right\} \delta x,
$$

for the attraction of a lamina of the cone. Also, if $\alpha$ be the semivertical angle of the cone, we have

$$
\frac{x}{\sqrt{\left(x^{2}+b^{2}\right)}}=\cos \alpha
$$

hence, the resultant attraction

$$
=2 \pi \rho(1-\cos \alpha) \int_{a}^{h} d x=2 \pi \rho(1-\cos \alpha) h ;
$$

where $h$ is the height of the cone.

It is easily seen that the same expression holds for the attraction of the frustum of a cone on a particle situated at the vertex of the complete cone, $h$ representing in this case the height of the frustum. 
If the cone be an oblique cone the base of which is any plane figure it is still true that the attraction of a frustum on a partiele at the vertex varies as the thickness of the fruatum. Consider two indefinitely thin parallel lamine at different distances from the vertex of such a cone, then the attractions of these lamina on the particle at the vertex will be the same. For take any indefinitely small element of area on the surface of one of the laminie, and let a conical surface be formed by straight lines which pass through the perimeter of this area and through the attracted particle; this conical surfince will intercept elements in the two lamina which are bounded by similar plane figures. Now, supposing the laminas of the same thickness, the masses of the elements will vary as the squares of their distances from the attracted particle, and thus they will exert egrual attractions on this particle. The same result holds for every corresponding pair of elements in the two lamina, and thus the two lamina exert on the particle at the vertex attractions which are equal in amount and coincident in direction. From this it follows that the attraction of a frostum varies as its thickness.

210. We have hitherto considered the attracting body to be of uniform density, but considerable variety may be introduced into the questions by various suppositions as to the law of density. Suppose, for instance, that in the case of the circular lamina in Art. 207 the density at any point of the lamina is $\phi(r)$, where $r$ is the distance of that point from the centre; $\phi(r)$ must then be put instead of $\rho$ in Art. 207 and must be placed under the integral sign. Therefure the attraction of the lamina will be

$$
2 \pi c k \int_{0}^{0} \frac{\phi(r) r d r}{\left(\sigma^{2}+r^{2}\right)} \text {. }
$$

If $\phi(r)=\frac{\sigma}{r}$, where $\sigma$ is a constant, the result is

$$
2 \pi c \kappa \sigma \int_{0}^{b} \frac{d r}{\left(c^{3}+r^{n}\right)} \text {, or } \frac{2 \pi x \sigma b}{c\left(c^{2}+b^{2}\right)} \text {. }
$$

211. To find the resultunt attraction of an assemblage of particles constituting a homegeneums spherical shell of very small thickness on a particle outside the shell. 
Let $C$ be the centre of the shell, $M$ any particle of it, $P$ the

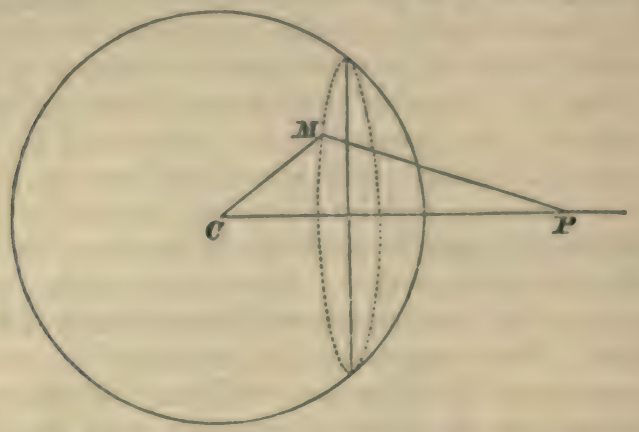

attracted particle. Let $C M=r, P I I=y, C P=c, \theta=$ the angle PCM, $\phi=$ the angle which the plane $P C M$ makes with the plane of the paper, $\delta r=$ the thickness of the shell, and let $\rho$ denote the density of the shell.

The volume of the elementary solid at $M$ is $r^{2} \sin \theta \delta r \delta \theta \delta \phi$ (see Art. 130). The attraction of the whole shell acts along $I^{\prime} C$; the attraction of the element at $M$ resolved along $P C$ is

$$
\frac{\rho r^{2} \sin \theta \delta r \delta \theta \delta \phi}{y^{3}} \frac{c-r \cos \theta}{y} .
$$

We shall eliminate $\theta$ from this expression by means of the equation

$$
y^{2}=c^{2}+r^{2}-2 r c \cos \theta ;
$$

therefore

$$
\begin{gathered}
\sin \theta \frac{d \theta}{d y}=\frac{y}{c r}, \\
c-r \cos \theta=\frac{y^{2}+c^{2}-r^{2}}{2 c} .
\end{gathered}
$$

Therefore the attracticn of $M$ on $P$ along $P C$

$$
=\frac{\rho r \delta r}{2 c^{8}}\left(1+\frac{c^{2}-r^{2}}{y^{3}}\right) \delta y \delta \phi .
$$


Ifence the resultant attraction of the whole shell

$$
\begin{aligned}
& =\frac{\rho r \delta r}{2 c^{2}} \int_{0.0}^{e 00} \int_{0}^{m}\left(1+\frac{c^{3}-r^{2}}{y^{2}}\right) d y d \phi=\frac{\pi \rho r \delta r}{c^{2}} \int_{\omega}^{\infty 00}\left(1+\frac{e^{2}-r^{2}}{y^{2}}\right) d y
\end{aligned}
$$

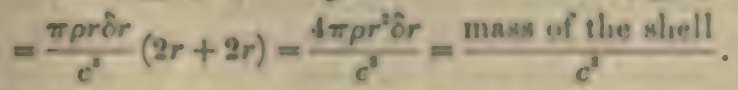

This result shews that the shell aftracts the particle at $P$ in the same manner as if the mass of the shell were condensed at its centre.

212. It follows from the preceding Article, that a sphere which is cither homogeneous or consists of concentric spherical shells of uniform density, attracts the particle at $P$ in the same manner as if the whole mass were collected at its centre.

213. To find the attraction of a homogeneous spherical shell of small thickness on a particle placed veithin is.

We must proceed as in Art. 211; but the limits of $y$ are in this case $r-c$ and $r+c$; hence the resultant attraction of the shell

$$
=\frac{\pi \rho r \delta r}{c^{3}} \int_{r-\infty}^{\text {the }}\left(1-\frac{r^{3}-c^{2}}{y^{2}}\right) d y=\frac{\pi \rho r \delta r}{c^{3}}(2 c-2 c)=0 .
$$

Therefore a particle within the shell is equally attracted in every direction.

Suppose a particle inside a homogenenus sphere at the distance $r$ from its centre; then by what has just been shewn all that portion of the sphere which is at a greater distance from the centre than the particle produces no effect on the particle. Also by Art. 211, the remainder of the sphere attracts the particle in the same manner as if the mass of the remainder were all collected at the centre of the sphere. Thus if $\rho$ be the density of the spphere the attraction on the particle is

$$
\frac{\frac{4}{3} \pi \rho r^{3}}{r^{2}} \text {, that is } \frac{4 \pi \rho r}{3} \text {. }
$$

Thus insile a homogeneus sphere the attraction naries as the distance from the centre.

214. The propositions respecting the attraction of a uniform spherical shell on an external or internal particle were 
given by Newton (Principia, Lib. 1. Prop. 70, 71). The result with respect to the internal particle was extended by Newton to the case of a shell bounded by sinilar and similarly situated spheroidal surfaces (Principia, Lib. 1. Prop. 91, Cor. 3). The proposition is also true when the shell is bounded by similar and similarly situated ellipsoidal surfaces, which we proceed to demonstrate in the method given by Newton for spheroidal surfaces.

215. If a shell of uniform density be bounded by two ellipsoital surfaces which are concentric, similar, and similarly situated, the resultant attraction on an internal particle vanishes.

Let the attracted particle $P$ be the vertex of an infinite series of right cones. Let $N M P M^{\prime} N^{\prime}$ and $n m P m^{\prime} n^{\prime}$ be two generating lines of one of these cones, and suppose the curves in the figure to represent the intersection of the surfaces of the shell by a plane containing these generating lines. The curves will be similar and similarly situated ellipses, and by a property of such ellipses,

$$
M N^{\prime}=M I^{\prime} N^{\prime} \text { and } m n=m^{\prime} n^{\prime} .
$$

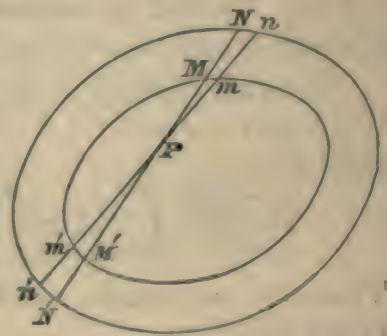

By taking the angle of the cone small enough, each of the two portions of the shell which it intereepts will be ultimately a frustum of a cone, and being of equal altitude and having a common vertical angle, they will exercise equal attractions on P. (See Art. 209.) Similar considerations hold with respect to each of the infinite series of cones of which $P$ is the vertex, and consequently the resultant attraction of the shell vanishes.

This result being true, whatever be the thickness of the shell, is true when the shell becomes indefinitely thin.

216. In a somewhat similar way we may establish the following proposition which is due to Poisson; the resultant attraction of an indefinitely thin shell bounded by two ellipsoidal surfaces which are concentric, similar, and similarly situated on an external particle is in the direction of the axis of the enveloping cone which has its vertex at the given particle. (Crelle's Journal, Vol. xII. p. 141.) Denote the external 
particle by $Q$; and suppose $P$ in the preceding figure to be the point where the axis of the enveloping cone intersects the plane of eontact of the cone and the ellipsoidal shell. Draw uny straight lines NaCMCN and mmmin as in the preceding figure. Lat $\mu$ denote the mass of the element $B / n$ and $\mu$ the mass of the element $M$ ' $n$ '.

The attraction of $\mu$ is equal to ${ }_{Q . M}$, and it acts along QM; the atiraction of $\mu^{\prime}$ is equal to $\frac{\mu^{\prime}}{Q . M^{\prime \prime}}$ and it acts along $Q . K^{\prime}$.

Now

$$
\frac{\mu}{P M}=\frac{\mu^{\prime}}{P M^{2}}
$$

and it is known that QM and QM' make oyual angles with QP (see Conic Sections, Chap. XV., last example); therefore

$$
\frac{P . M}{Q . M}=\frac{P . I^{\circ}}{Q . M^{\prime}}
$$

and thereforo

$$
\frac{\mu}{Q M^{\circ}}=\frac{\mu^{\prime}}{Q M^{\mathrm{a}}} .
$$

Thus the elements $\mu$ and $\mu^{\prime}$ exert equal aftractions on $Q$; and since the directions of these aftractions make equal angles with $Q P$, the resultant attraction of these two elements acts along QP. A similar result holds for every pair of elements into which the ellipsoidal shell may be decomposed; and thus the proposition follows. It appears from the eourse of the demonstration that any plane through $P$ divides the shell into two parts which exercise equal attractions on $Q$.

It follows from this result, by proceeding to the limit, that the resultant attraction of the indefinitely thin shell on a particle in contact with the external surface is in the direction of the normal to the surface at the point of contact.

We shall now give in the next two Articles some propositions which will serve as exercises; the approximate results which we shall obtain may be subsequently verified by an exact investigation. (See Art. 226.)

217. To find the altraction of a hommeneous oblate spheroid of small excentricity on a particle at its pole.

Let $2 c$ be the length of the minor axis and $2 a$ that of the major axis of the generating ellipse. The spheroid may be 
supposed made up of a concentric sphere, the radius of which is $c$, and an exterior shell; we shall calculate the attractions of these portions separately.

Let a section be made of the sphere and spheroid by a plane perpendicular to the axis of revolution of the spheroid at a distance $x$ from the attracted particle. This plane cuts the sphere and spheroid in concentric circles; the area of the former being $\pi y^{2}$ and of the latter $\frac{\pi a^{2} y^{2}}{c^{3}}$, where $y^{2}=2 c x-x^{2}$; the difference of these areas is $\pi\left(\frac{a^{2}}{c^{2}}-1\right) y^{2}$. If a section be made by a second plane, parallel to the former and at a distance $\delta x$ from it, the volume of the portion of the shell intercepted between the planes will be $\pi\left(\frac{a^{2}}{c^{2}}-1\right) y^{2} \delta x$. The distance of every particle of the annulus thus formed from the attracted particle is approximately $\sqrt{ }(2 c x)$, and, as the resultant attraction of the annulus will act along the axis of the spheroid, it will, approximately,

$$
\begin{aligned}
& =\pi \rho\left(\frac{a^{2}}{c^{2}}-1\right) \frac{x}{\sqrt{(2 c x)}} \frac{y^{2} \delta x}{2 c x} \\
& =\pi \rho\left(\frac{a^{2}}{c^{2}}-1\right) \frac{2 c x^{4}-x^{2}}{(2 c)^{\frac{1}{3}}} \delta x .
\end{aligned}
$$

Therefore the resultant attraction of the shell

$$
=\frac{\pi \rho\left(a^{2}-c^{2}\right)}{2^{\frac{1}{2}} c^{\frac{2}{3}}} \int_{0}^{20}\left(2 c x^{\frac{1}{4}}-x^{3}\right) d x=\frac{8 \pi \rho\left(a^{2}-c^{2}\right)}{15 c} .
$$

If we suppose $c=a(1-\epsilon), \epsilon$ being very small, we have

$$
a^{2}-c^{2}=2 c^{2} \epsilon \text { approximately; }
$$

therefore the resultant attraction of the shell

$$
=\frac{16 \pi \rho e c}{15} \text {. }
$$

Also the attraction of the sphere on the particle, by Art. 212,

$$
=\frac{4}{3} \pi \rho c \text {; }
$$


therefore the attraction of the sphererid on the particle

$$
-1 \pi \rho(1+f \epsilon) c
$$

218. To find the altraction of a homogeneous oblale splieroid of amall excentricity on a particle at its equator.

Let $2 e$ be the length of the minor axis, and $2 a$ that of the major axis of the generating ellipse. The spheroid may be supposed to be the difference between a concentric sphere of radius $a$ and a shell, and the attractions of the sphere and shell may be separately calculated. Let a section be made of the sphere and spheroid by a plane perpendicular to the straight line joining the attracted particle with the common centre of the sphere and spheroid, and at a distance $x$ from the attracted particle; this plane will cut the sphere in a circle the aren of which is $\pi y^{\prime}$, where $y^{3}=2 a x-x^{3}$, and it will cut the spheroid in an ellipse of which the semi-axes are respectively $y$ and $\frac{c y}{a}$, and the aren of which is therefore $\frac{\pi c}{a} y^{2}$. The difference of the two areas is $\pi\left(1-\frac{c}{a}\right) y$. If a section be maile by a second plane parallel to the former. and at a distance $\delta x$ from it, the volume of the portion of the shell intercepted between the planes will be $\pi\left(1-\frac{c}{a}\right) y^{3} \delta x$. The diatance of every particle of the annulus thus formed from the attracted particle is approximately $\sqrt{ }(2 a x)$; and as the resultant attraction of the annulus will act along the straight line joining the attracted particle with the centre, it will alproximately

$$
\begin{aligned}
& =\pi \rho\left(1-\frac{c}{a}\right) \frac{x}{\sqrt{(2 a x)}} \frac{y^{2} \delta x}{2 a x} \\
& =\pi \rho\left(1-\frac{c}{a}\right) \frac{2 a x^{\prime}-x^{\prime}}{(2 a)^{\prime}} \delta x .
\end{aligned}
$$

Therefore the resultant attraction of the shell

$$
\begin{aligned}
& =\frac{\pi \rho(a-c)}{2^{i} a^{3}} \int_{0}^{m}\left(2 a x^{b}-x^{l}\right) d x=\frac{8 \pi \rho(a-c)}{15} \\
& =\frac{8 \pi \rho a e}{13} \text {, if } c=a(1-c) .
\end{aligned}
$$


Also the attraction of the sphere, by Art. 212,

$$
=\frac{f}{s} \pi \rho a \text {; }
$$

therefore the attraction of the spheroid on the particle

$$
\begin{aligned}
& =\frac{4}{3} \pi \rho a-\frac{8 \pi \rho \pi \epsilon}{15}=\frac{4}{3} \pi \rho\left(1-\frac{2}{5} \epsilon\right) a \\
& =\frac{4}{3} \pi \rho\left(1+\frac{8}{3} \epsilon\right) c .
\end{aligned}
$$

In the same manner it might be shewn that the attractions of a homogeneous prolate spheroid of small excentricity on particles at the pole and equator are respectively

$$
\frac{4}{3} \pi \rho\left(1-\frac{4}{6} \epsilon\right) c \text { and } \frac{4}{3} \pi \rho\left(1-\frac{3}{5} \epsilon\right) c,
$$

$2 c$ being the axis of revolution of the spheroid, and

$$
a=c(1-\varepsilon) \text {. }
$$

219. One more example may be given. It is sometimes useful to compare the attraction exerted by the Farth on a particle at the top of a mountain with the attraction exerted by the Earth on the same particle at the ordinary level of the Earth's surface. The investigation is given by Poisson, (Mécanique, Tom. I. pp. 492-496). Let $r$ denote the Earth's radius, $x$ the height of the mountain, $g$ the attraction of the Farth on a particle of a unit of mass at the ordinary level of the Earth's surface. If there were no mountain the attraction of the Earth on the particle at a distance $x$ from its surface would be $g \frac{r^{3}}{(r+x)^{8}}$ : we have then to add to this expression the attraction exerted by the mountain itself. Suppose the mountain to be of uniform density $\rho$, and consider it to be cylindrical in shape, and the particle to be at the centre of its upper surface; then by Art. 208 the resultant attraction is

$$
2 \pi \rho\left\{x-\sqrt{ }\left(x^{2}+b^{2}\right)+b\right\},
$$

where $b$ is the radius of the cylinder. If $b$ is so large in comparison with $x$ that the square of $\frac{x}{b}$ can be neglected, this 
exprossion reduces to $2 \pi p x$. Thus if $g$ denote the attraction at the top of the mountain

$$
g^{\prime}=\frac{g r^{2}}{(r+x)^{2}}+2 \pi p r .
$$

Let $\sigma$ denote the mean density of the Farth, so that the mass of the Farth is $\frac{4 \pi \sigma r^{2}}{3}$; then

thus

$$
\begin{gathered}
g=\frac{4 \pi \sigma r^{3}}{3 r^{2}}=\frac{4 \pi \sigma r}{3} ; \\
g^{\prime}=g\left\{\frac{r^{2}}{\left(r+x_{i}\right.}+\frac{3 \rho \sigma}{2 \sigma r}\right\} .
\end{gathered}
$$

Now the mean density of the Farth is known to be about five and a half times that of water, and from what may be conjectured of the density of matter at the Earth's surface, we may suppose $\frac{\rho}{\sigma}=\frac{1}{2}$. And

$$
\frac{r^{2}}{(r+x)^{i}}-\left(1-\frac{x}{r}\right)^{-3}=1-\frac{2 x}{r} \text { approximately; }
$$

thus

$$
g^{\prime}=g\left(1-\frac{2 x}{r}+\frac{8 x}{4 r}\right)=g\left(1-\frac{8 x}{4 r}\right) .
$$

How far the approximations made in this Article are allowable might be difficult to estimate; from Artecle 207 , it appears that in taking $2 \pi \rho x$ to represent the attraction of the mountain, we do in fact make the mountain to consist of a uniform plate of finite thickness $x$, but of infinite extent.

For investigations relating to the attractions of mountains the student may consult Pratt's treatise on Attractions... and the figure of the Earth.

Wo have hitherto confinel ourselves to simple examples of the ordinary law of attraction; we now proceed to consider some other laws of attraction, and also some more complex eases of the ordinary law.

220. If the particles of a bealy aetrane will a force varying as the prostuct of the mesas inte the disternce, the resulteant attraction of the lenty is the same as if the schule mass of the butly ecere collected at its centre of gravity. 
Take the centre of gravity of the attracting body as the origin of co-ordinates, and let $a, b, c$ be the co-ordinates of the attracted particle. Divide the attracting body into indefinitely small elements; let $x, y, z$ be the co-ordinates of an element, $m$ its mass, and $r$ its distance from the attracted particle. Then the attraction of this element is $m r$, and by resolving it parallel to the co-ordinate axes, we obtain

$$
m r \cdot \frac{a-x}{r} ; m r \cdot \frac{b-y}{r}, m r \cdot \frac{c-z}{r},
$$

respectively. Hence, if $X, Y, Z$ denote the resolved parts of the whole attraction, we have

$$
X=\Sigma m(a-x), \quad Y=\Sigma m(b-y), \quad Z=\Sigma m(c-z) .
$$

But, since the origin is the centre of gravity of the attracting body, we have

$$
\mathbf{\Sigma} m x=0, \quad \Sigma m y=0, \quad \Sigma m z=0 ;
$$

therefore $\quad X=a \Sigma m, \quad Y=b \Sigma m, \quad Z=c \Sigma m$.

But these expressions are the resolved attractions of a mass $\Sigma_{m}$ placed at the origin, which establishes the proposition.

221. To find the attraction of a homogeneous spherical shell on a particle vithout it; the lavo of attraction being represented by $\phi(y)$, where $y$ is the distance.

If we proceed as in $\Lambda$ rt. 211 , we find the resultant attraction of the shell on $P$ along $P C$

$$
=\frac{\pi \rho r \delta r}{c^{2}} \int_{c \rightarrow r}^{c t r}\left(y^{2}+c^{2}-r^{2}\right) \phi(y) d y .
$$

Suppose

$$
\int \phi(y) d y=\phi_{1}(y)
$$

and

$$
\int y \phi_{1}(y) d y=\psi(y) \text {. }
$$

Then, integrating by parts, we have

$$
\begin{aligned}
\int\left(y^{2}+c^{2}-r^{2}\right) \phi(y) d y & =\left(y^{2}+c^{2}-r^{2}\right) \phi_{1}(y)-2 \int y \phi_{1}(y) d y \\
& =\left(y^{2}+c^{2}-r^{2}\right) \phi_{1}(y)-2 \psi(y) ;
\end{aligned}
$$


therefore

$$
\frac{\pi \rho r \delta r}{e^{3}} \int_{a, p}^{m p}\left(y^{2}+c^{2}-r^{2}\right) \phi(y) d y
$$

$=2 \pi \rho r o ̂ r\left\{\frac{c+r}{c} \phi_{1}(c+r)-\frac{c-r}{c} \phi_{1}(c-r)-\frac{1}{c} \psi(c+r)+\frac{1}{c} \psi(c-r)\right\}$

$=2 \pi \rho r \delta r \frac{d}{d c}\left\{\frac{\psi(c+r)-\psi(c-r)}{c}\right\}$.

This last form is introduced merely as an analytical artifice to simplify the expression.

222. To find the attruction of the shell on an internal particle.

The calculation is the same as in the lnst Article, except that the limits of $y$ are $r-c$ and $r+c$. Hence, the attraction of the shell

$=2 \pi \rho r \delta r\left\{\frac{r+c}{c} \phi_{1}(r+c)+\frac{r-c}{c} \phi_{1}(r-c)-\frac{1}{c} \psi(r+c)+\frac{1}{c} \psi(r-c)\right\}$

$=2 \pi \rho r \delta r \frac{d}{d e}\left\{\frac{\psi(r+c)-\psi(r-c)}{c}\right\}$.

223. The formula of the preceding two Articles will give the attraction when the law of attraction is known.

Ex. 1. Iat $\phi(r)=\frac{1}{r^{2}}$; therefore $\phi_{3}(r)=-\frac{1}{r}+A$,

$$
\psi(r)=-r+\frac{1}{2} A r^{2}+B
$$

$A$ and $B$ being constants.

Therefore the attraction on an external particle

$$
\begin{aligned}
& =2 \pi \rho r \delta r \frac{d}{d c}\left\{\frac{-4 r+A(0+r)^{2}-A(c-r)^{2}}{2 c}\right\} \\
& =2 \pi \rho r \delta r \frac{d}{d c}\left(-\frac{2 r}{c}+2 A r\right)=\frac{4 \pi \rho r^{3} \delta r}{c^{2}},(A r t 211) .
\end{aligned}
$$


The attraction on an internal particle

$$
\begin{aligned}
& =2 \pi \rho r \delta r \frac{d}{d c}\left\{\frac{-4 c+A(r+c)^{2}-A(r-c)^{2}}{2 c}\right\} \\
& =2 \pi \rho r \delta r \frac{d}{d c}\{-2+2 A r\}=0, \text { (Art. 213). }
\end{aligned}
$$

\section{Ex. 2. Let $\phi(r)=r$;}

therefore $\phi_{1}(r)=\frac{1}{2} r^{8}+A, \psi(r)=\frac{1}{8} r^{4}+\frac{1}{2} A r^{2}+B$.

The attraction on an external particle

$$
\begin{aligned}
& =2 \pi \rho r \delta r \frac{d}{d c}\left\{\frac{(c+r)^{4}-(c-r)^{4}+4 A(c+r)^{2}-4 A(c-r)^{2}}{8 c}\right\} \\
& =2 \pi \rho r \delta r \frac{d}{d c}\left\{c^{2} r+r^{3}+2 A r\right\} \\
& =4 \pi \rho r^{2} c \delta r=\operatorname{mass} \times c .
\end{aligned}
$$

The attraction therefore is the same as if the shell were collected at its centre. This property we discovered for the law of the inverse square. We shall now ascertain whether there are any other laws which give the same property.

224. To find what laws of attraction allow us to suppose a spherical shell condensed into its centre when attracting an external particle.

Let $\phi(r)$ be the law of force; then, if $c$ be the distance of the centre of the shell from the attracted particle, $r$ the radius of the shell, and $\psi(r)=\int\left\{r \int \phi(r) d r\right\} d r$, the attraction of the shell

$$
=2 \pi \rho r \delta r \frac{d}{d c}\left\{\frac{\psi(c+r)-\psi(c-r)}{c}\right\} .
$$

But if the shell be condensed into its centre, the attraction

$$
=4 \pi \rho r^{2} \delta r \phi(c) \text {; }
$$

therefore

$$
\frac{d}{d c}\left\{\frac{\psi(c+r)-\psi(c-r)}{c}\right\}=2 r \phi(c) \text {. }
$$


Fxpand $\psi(c+r)$ and $\psi(e-r)$ in powers of $r$; then using $\psi^{\prime}(e)$ for $\frac{d \psi(e)}{d e}$, Sc., we have

$$
\begin{aligned}
2 r \phi(c) & =2 \frac{d}{d c}\left\{\frac{r}{c} \psi^{\prime}(c)+\frac{r^{2}}{c[3} \psi^{\prime \prime \prime}(c)+\ldots\right\} \\
& =2 r \phi(c)+2 \frac{d}{d c}\left\{\frac{r^{2}}{c L^{3}} \psi^{\prime \prime}(c)+\ldots\right\} ; \\
& \frac{d}{d c}\left\{\frac{r^{2}}{c[3} \psi^{\prime \prime \prime}(c)+\ldots\right\}=0,
\end{aligned}
$$

therefore

whatever $r$ may be; therefore

$$
\frac{d}{d c}\left\{\frac{\psi^{(0)}(c)}{c}\right\}=0, \quad \frac{d}{d c}\left\{\frac{\psi^{(c)}(c)}{c}\right\}=0, d c .
$$

But

$$
\psi^{\prime}(c)=c\lceil\phi(c) d e \text {; }
$$

therefore

$$
\psi^{\prime \prime}(c)=\int \phi(c) d c+c \phi(c) \text {; }
$$

therefore

$$
\psi^{\prime \prime \prime}(c)=2 \phi(c)+c \phi^{\prime}(r) \text {. }
$$

Therefore, by the first of the above equations of condition for $\psi(c)$,

$$
\frac{2 \phi(c)}{c}+\phi^{\prime}(c)=a \text { constant. }
$$

Put $3 A$ for this constant; multiply both sides of the equation by $c^{3}$ and integrate; thus

$$
\begin{gathered}
c^{2} \phi(c)=A c^{2}+B ; \\
\phi(c)=A c+\frac{B}{c^{3}} .
\end{gathered}
$$

therefore

This value satisfies all the other equations of condition for $\psi$ (c): therefore the required laws of attraction are those of the direct distance, the inverse square, and a law compounded of these.

225. To find for what lanes the shell attracts an internal particle equally in every direction. 
When this is the case,

$$
\frac{d}{d c}\left\{\frac{\psi(r+c)-\psi(r-c)}{c}\right\}=0 ;
$$

therefore

$$
\psi^{\prime}(r)+\frac{c^{2}}{[3} \psi^{\prime \prime \prime}(r)+\ldots=\Lambda,
$$

whatever $c$ is, $A$ being a constant independent of $c$; therefore

$$
\psi^{\prime}(r)=A, \quad \psi^{\prime \prime \prime}(r)=0, \& c .
$$

From the second condition, we have

$$
\psi(r)=B+B^{\prime} r+B^{\prime \prime} r^{2},
$$

where $B, B^{\prime}$, and $B^{\prime \prime}$ are constants.

Hence

$$
\begin{aligned}
\psi^{\prime}(r) \text { or } r \int \phi(r) d r & =B^{\prime}+2 B^{\prime \prime} r ; \\
\int \phi(r) d r & =\frac{B^{\prime}}{r}+2 B^{\prime \prime} \\
\phi(r) & =-\frac{B^{\prime}}{r^{2}}:
\end{aligned}
$$

with this value of $\phi(r)$ all the other equations of condition are satisfied; hence the only law which satisfies the condition is that of the inverse square.

226. To find the attraction of a homogeneous oblate spheroid on a particle within its mass, the law of attraction being that of the inverse square of the distance.

Let $a$ and $c$ be the semi-axes, $a$ being greater than $c$; and let the equation to the spheroid referred to its centre as origin be

$$
\frac{x^{2}+y^{2}}{a^{2}}+\frac{x^{2}}{c^{2}}=1
$$

Let $f, g, h$ be the co-ordinates of the attracted particle; $r$ the distance from the attracted particle of any point of the attracting mass; $\theta$ the angle which $r$ makes with a straight line parallel to the axis of $z ; \phi$ the angle which the plane 
taining $r$ and a straight line through the point $(f, g, h)$ paralles to the axis of a makes with the plane of $(x, z)$. The volume of an element of the attracting mass

$$
\text { - r } \sin 080 \delta \phi \delta 8 \text {, }
$$

as in Art. 130. Iat $\rho$ be the density of the sppheroid; then the atfraction of this clement on the attracted particle is $\rho \sin \theta \delta \theta \delta \phi \delta r$; and the resolved parts of this parallel to the axies of $x_{1} y_{1} z_{1}$, are

$\rho \sin ^{2} \theta \cos \phi \delta \theta \delta \phi \delta r, \quad \rho \sin ^{2} \theta \sin \phi \delta \theta \delta \phi \delta r$,

$$
\text { and } \rho \sin \theta \cos \theta \delta \theta \delta \phi \delta r \text {, }
$$

respectively. Ilence the attractions of the whole spheroid will be found by integrating these expressions between proper limits. We proceed to find these limits.

In equation (1) pat

$$
\begin{aligned}
& f+r \sin \theta \cos \phi \text { for } x, \\
& g+r \sin \theta \sin \phi \text { for } y, \\
& h+r \cos \theta \quad \text { for } z ;
\end{aligned}
$$

then the equation to the spheroid becomes

$$
\frac{(f+r \sin \theta \cos \phi)^{2}+(g+r \sin \theta \sin \phi)^{2}}{a^{2}}+\frac{(h+r \cos \theta)^{2}}{c^{2}}=1,
$$

or $r^{2}\left\{\frac{\sin ^{2} \theta}{a^{3}}+\frac{\cos ^{3} \theta}{c^{3}}\right\}+2 r\left\{\frac{f \sin \theta \cos \phi+g \sin \theta \sin \phi}{a^{3}}+\frac{h \cos \theta}{c^{3}}\right\}$

$$
=1-\frac{f^{2}+g^{2}}{a^{2}}-\frac{h^{2}}{c^{3}} \text {. }
$$

Put

$$
\frac{\sin ^{2} \theta}{a^{4}}+\frac{\cos ^{2} \theta}{\sigma^{3}}=K \text {, }
$$

$\frac{f \sin \theta \cos \phi+g \sin \theta \sin \phi}{a^{2}}+\frac{h \cos \theta}{\sigma^{2}}=F$,

$$
F^{2}+K\left(1-\frac{f^{2}+g^{2}}{a^{3}}-\frac{h^{2}}{e}\right)=\Pi \text {; }
$$

then

$$
K^{-2} r^{2}+2 K F r+F^{2}=I I
$$

T. 8. 
Equation (2) will give two values for $r$, one positive and the other negative; these values we may denote by $r_{1}$ and $-r_{2}$, where

$$
r_{1}=\frac{-F+\sqrt{ } H}{K}, \quad r_{2}=\frac{F+\sqrt{ } H}{K} .
$$

Hence to find the whole attraction of the spheroid parallel to the axis of $x$, we first integrate the expression

\section{$\rho \sin ^{2} \theta \cos \phi \delta \theta \delta \phi \delta r$}

with respect to $r$ between the limits $r=0$ and $r=r_{1}$, and also between the limits $r=0$ and $r=r_{3}$, and take the difference; we thus obtain

$$
\rho \sin ^{2} \theta \cos \phi\left(r_{2}-r_{1}\right) \delta \theta \delta \phi ;
$$

this must be integrated between 0 and $\pi$ for $\phi$, and 0 and $\pi$ for $\theta$. If $A$ denote the whole attraction parallel to the axis of $x$, acting towards the origin, we have then

$$
A=2 \rho \int_{0}^{\pi} \int_{0}^{\pi} \frac{F}{K} \sin ^{2} \theta \cos \phi d \theta d \phi .
$$

We may simplify this expression by omitting those terms which vanish by the principles of the Integral Calculus; thus

$$
\begin{aligned}
A & =2 f \rho c^{2} \int_{0}^{\pi} \int_{0}^{\pi} \frac{\sin ^{2} \theta \cos ^{2} \phi d \theta d \phi}{c^{2} \sin ^{2} \theta+a^{2} \cos ^{2} \theta} \\
& =\pi f \rho c^{2} \int_{0}^{\pi} \frac{\sin ^{3} \theta d \theta}{c^{2} \sin ^{2} \theta+a^{2} \cos ^{2} \theta} \\
& =\pi f \rho c^{2} \int_{0}^{\pi} \frac{\left(1-\cos ^{2} \theta\right) \sin \theta d \theta}{c^{2}+\left(a^{2}-c^{2}\right) \cos ^{2} \theta} \\
& =\frac{\pi f \rho c^{2}}{a^{2}-c^{8}} \int_{0}^{\pi}\left\{\frac{a^{2} \sin \theta}{c^{3}+\left(a^{2}-c^{2}\right) \cos ^{2} \theta}-\sin \theta\right\} d \theta \\
& =\frac{2 \pi f \rho c^{2}}{a^{2}-c^{2}}\left\{\frac{a^{2}}{c \sqrt{ }^{\prime}\left(a^{2}-c^{2}\right)} \tan ^{-1} \frac{\sqrt{\left(a^{2}-c^{2}\right)}}{c}-1\right\} .
\end{aligned}
$$

Let $c^{2}=a^{3}\left(1-e^{2}\right)$; then the result may be written

$$
A=2 \pi f \rho\left\{\frac{\sqrt{ }\left(1-e^{2}\right)}{e^{3}} \sin ^{-1} e-\frac{1-e^{2}}{e^{z}}\right\} .
$$


ATTBACTION. OBLATE 8PIEROND.

In the same manner, if $B$ denote the whole attraction parallel to the axis of $y$.

$$
B=2 \pi g \rho\left\{\frac{\sqrt{ }\left(1-e^{2}\right)}{e^{3}} \sin ^{-1} e-\frac{1-e^{e}}{e^{3}}\right\} .
$$

Iet $C$ denote the whole attraction parallel to the axis of z, then

$$
\begin{aligned}
& C=2 \rho \int_{0}^{\infty} \int_{0}^{\theta} \frac{F}{K} \sin \theta \cos \theta d \theta d \phi \\
& =2 h \rho a^{2} \int_{0}^{\pi} \int_{0}^{\pi} \frac{\sin \theta \cos ^{2} \theta d \theta d \phi}{c^{3} \sin ^{2} \theta+a^{2} \cos ^{2} \theta} \\
& =\frac{2 \pi l \rho a^{2}}{a^{2}-c^{2}} \int_{0}^{*}\left\{\sin \theta-\frac{c^{2} \sin \theta}{c^{3}+\left(a^{2}-c^{2}\right) \cos ^{2} \theta}\right\} d \theta \\
& =\frac{4 \pi h \rho a^{2}}{a^{3}-c^{2}}\left\{1-\frac{c}{\sqrt{\left(a^{3}-c^{2}\right)}} \tan ^{-1} \frac{\sqrt{\left(a^{2}-c^{2}\right)}}{c}\right\} \\
& =4 \pi h \rho\left\{\frac{1}{e^{2}}-\frac{\sqrt{\left(1-e^{2}\right)}}{e^{3}} \sin ^{-1} e\right\} \text {. }
\end{aligned}
$$

If the spheroid be prolate $a$ is less than $c$. It may be shewn then that

$$
\begin{aligned}
& A=\frac{2 \pi f \rho c^{2}}{c^{3}-a^{2}}\left\{1-\frac{a^{2}}{c \sqrt{\left(c^{2}-a^{3}\right)}} \log \frac{c+\sqrt{\left(c^{2}-a^{2}\right)}}{a}\right\}, \\
& B=\frac{2 \pi g c^{2}}{c^{3}-a^{2}}\left\{1-\frac{a^{2}}{c \sqrt{\left(c^{3}-a^{2}\right)}} \log \frac{c+\sqrt{ }\left(c^{2}-a^{3}\right)}{u}\right\} \text {, } \\
& C=\frac{4 \pi h p a^{3}}{c^{2}-a^{2}}\left\{\frac{c}{\sqrt{\left(c^{3}-a^{2}\right)}} \log \frac{c+\sqrt{\left(c^{2}-a^{2}\right)}}{a}-1\right\} .
\end{aligned}
$$

It may be noticed that in both cases

$$
\frac{A}{f}+\frac{B}{g}+\frac{C}{h}=4 \pi \rho
$$

227. From the expressions in the preceding Article we see that the attraction is independent of the magnitude of the spheroid and depends solely upon the excentricity. 
Hence the attraction of the spheroid similar to the given one and passing through the attracted particle, is the same as that of any other similar and similarly situated concentric spheroid comprising the attracted particle in its mass. Hence a spheroidal shell the surfaces of which are similar, similarly situated, and concentric, attracts a particle within it equally in all directions. This has been already established; see Art. 215.

If we put the ellipticity of the spheroid $=\epsilon$, and suppose e very small so that we may neglect its square, we have for the oblate spheroid, since $c=a(1-\varepsilon)$,

$$
e^{2}=1-\frac{c^{3}}{a^{3}}=1-(1-\epsilon)^{2}=2 \epsilon \text { approximately. }
$$

After expansion and reduction we shall obtain approximately

$$
\begin{aligned}
& A=\frac{f}{z} \pi \rho(1-q \epsilon) f, \\
& B=\frac{s}{3} \pi \rho\left(1-\frac{q}{\xi} \epsilon\right) g, \\
& C=\frac{4}{3} \pi \rho\left(1+\frac{f}{\delta} \epsilon\right) h ;
\end{aligned}
$$

For the prolate spheroid, since $a=c(1-\epsilon)$,

$$
e^{2}=1-\frac{a^{2}}{c^{3}}=1-(1-\epsilon)^{2}=2 \epsilon .
$$

After expansion and reduction we shall obtain approximately

$$
\begin{aligned}
& A=\frac{4}{3} \pi \rho\left(1+\frac{2}{5} \epsilon\right) f, \\
& B=\frac{4}{3} \pi \rho\left(1+\frac{2}{5} \epsilon\right) g, \\
& C=\frac{4}{3} \pi \rho\left(1-\frac{f}{5} \epsilon\right) h .
\end{aligned}
$$

228. If instead of the spheroid we take an ellipsoid whose semi-axes are $a, b, c$, it may be shewn that

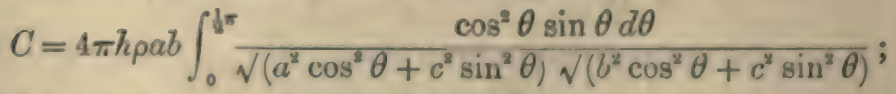

and the values of $A$ and $B$ may be found by symmetrical changes in the letters $a, b, c$ and $f, g, h$.

If we change $a, b, c$ into $a(1+n), b(1+n), c(1+n)$ respectively, the expression for $C$ remains unchanged; and so also the expressions for $A$ and $B$ remain unchanged. 'This 
shews that a shell of any thicknesa, the internal and external boundaries of which are similar and similarly situated concentrie ellipsoids, exerts no attraction on a particle within the inner boundary. This has been already eistablished; see Art. 215.

229. Suppose we require the attraction of a spheroid on an external particle.

In the equation (2) of Art. 226, we shall now have $F^{0}-H$ a positive quantity, and the two roots of that quadratic equastion will have the rame sign. Hence we shall find

$$
A=2 \rho \iint \frac{\sqrt{ } I I}{h^{\prime}} \sin ^{2} \theta \cos \phi d \phi d \theta \text {. }
$$

The limits of the integration with respect to $\theta$ will involve $\phi$, for these limits will be found by putting $\|=0$, and this leads to the following quadratie equation for determining $\tan \theta$,

$$
\begin{aligned}
& \tan ^{2} \theta\left\{\left(\frac{i \cos \phi+g \sin \phi}{a^{2}}\right)^{2}+\frac{1}{a^{2}}\left(1-\frac{f^{2}+g^{2}}{a^{3}}-\frac{h^{2}}{c^{2}}\right)\right\} \\
& +\frac{2 h \tan \theta}{c^{3}} \cdot \frac{f \cos \phi+g \sin \phi}{a^{2}}+\frac{1}{c^{2}}\left(1-\frac{f^{2}+g^{2}}{a^{3}}\right)=0 .
\end{aligned}
$$

Then the limits of $\phi$ are to be determined from the condition that the values of $\tan \theta$ furnished by this quadratic equation must be equal; this leads after some reduction to the following equation for determining the limits of $\phi$,

$$
(f \cos \phi+g \sin \phi)^{2}=f^{2}+g^{2}-a^{2} .
$$

It is however unnecessary to proceed with these complicated integrations, for we can obtain the result indirectly by means of Irory's theorem, which furnishes a relation between the attractions of ellipacids on external and interval particles; this sheorem will be true for spheroids as they are included among ellipsoils, and since the attraction of a spheroid on an internal prarticle has been already found, the theorem will enable us to determine the attraction of a splieroid on an cxternal particle.

2:30. We shall require a preliminary definition and proposition before we give Irory's theorem. 
Corresponding points on two ellipsoids are points whose co-ordinates are proportional to the axes to which they are respectively parallel.

In corfocal ellipsoids the distance between two points, one on each ellipsoid, is equal to the distance between their corresponding points.

Let $(x, y, z)$ and $(\xi, \eta, \xi)$ denote two points $P$ and $Q$ on an ellipsoid whose semi-axes are $a, b, c$; then the corresponding points $P^{\prime}$ and $Q^{\prime}$ on an ellipsoid whose semi-axes are $a^{\prime}, b^{\prime}, c^{\prime}$, will be denoted by

$$
\left(\frac{a^{\prime} x}{a}, \frac{b^{\prime} y}{b}, \frac{c^{\prime} z}{c}\right) \text { and }\left(\frac{a^{\prime} \xi}{a}, \frac{b^{\prime} \eta}{b}, \frac{c^{\prime} \zeta}{c}\right) \text {. }
$$

Thus

$$
\begin{aligned}
& P Q^{\prime 2}=\left(x-\frac{a^{\prime} \xi}{a}\right)^{2}+\left(y-\frac{h^{\prime} \eta}{b}\right)^{2}+\left(z-\frac{c^{\prime} \zeta}{c}\right)^{2}, \\
& P^{\prime} Q^{2}=\left(\xi-\frac{a^{\prime} x}{a}\right)^{2}+\left(\eta-\frac{h^{\prime} y}{b}\right)^{2}+\left(\zeta-\frac{c^{\prime} z}{c}\right)^{2} .
\end{aligned}
$$

Therefore $P Q^{\prime 2}-P^{\prime} Q^{2}=$

$$
\begin{gathered}
\left(x^{2}-\xi^{2}\right)\left(1-\frac{a^{\prime 2}}{a^{2}}\right)+\left(y^{2}-\eta^{2}\right)\left(1-\frac{b^{\prime 2}}{b^{2}}\right)+\left(z^{2}-\xi^{2}\right)\left(1-\frac{c^{\prime 2}}{c^{2}}\right) \\
=\left(a^{2}-a^{2}\right)\left\{\frac{x^{2}}{a^{2}}-\frac{\xi^{2}}{a^{2}}+\frac{y^{2}}{b^{2}}-\frac{\eta^{2}}{b^{2}}+\frac{z^{2}}{c^{2}}-\frac{\zeta^{2}}{c^{2}}\right\},
\end{gathered}
$$

because the ellipsoids are confocal.

But

$$
\frac{x^{2}}{a^{2}}+\frac{y^{2}}{b^{2}}+\frac{z^{2}}{c^{2}}=1=\frac{x^{\prime 2}}{a^{2}}+\frac{y^{\prime 2}}{b^{2}}+\frac{z^{\prime 2}}{c^{2}} ;
$$

therefore

$$
\begin{gathered}
P Q^{\prime 2}-P^{\prime} Q^{2}=0 ; \\
P Q^{\prime}=P^{\prime} Q .
\end{gathered}
$$

thus

Ivory's Theorem. The attraction of an ellipsoid on a particle on the surface of a confocal ellipsoid resolved parallel to an axis is to the attraction of the second ellipsoid on the corresponding point on the surface of the first ellipsoid, so 
resolved, as the protuluct of the other twoo axes of the first ellipsoid is to the correspeneiling prouluet in the second ellipioid? the thoo ellipsoids boing homejemens and of the same density.

Let $a, b, c$ be the m-mi-nxes of the first elliponid; $a^{\prime}, b^{\prime}, c^{\prime}$ those of the second. Lat $(j, g, h)$ denote a point on the surface of the first ellipeoil; $(f, g, h)$ the corresponding point on the surface of the second ellipinoid.

The attraction of the first ellipsoid on a particle at $\left(f^{\prime}, g^{\prime}, k^{\prime}\right)$ resolved parallel to the axis of $x$ is

$$
\iiint \mu \frac{x-f^{p}}{r} \phi(r) d x d y d z,
$$

where

$$
r^{\prime}=\left(x-f^{\prime}\right)^{2}+\left(y-g^{\prime}\right)^{\prime}+\left(z-h^{\prime}\right)^{2} \text {, }
$$

and the law of attraction is represented by $\phi(r) ; \mu$ is a constant: the integration is to extend throughout the volume of the first ellipsoid.

Iet $f \phi(r) d r=\psi(r)$. Integrate with respect to $x$; and let $r$ and $r_{3}$ denote the values of $r$ at the extremities of a chord of the ellipsoid parallel to the axis of $x$. 'Thus the resolved attraction is

$$
\iint\left[\psi\left(r_{2}\right)-\psi\left(r_{3}\right)\right] d y d z \text {. }
$$

In the same way the resolved attraction of the second ellipsoid on the corresponding point on the surface of the first ellipsoid may be expressed by

$$
\iint\left\{\psi\left(r^{\prime}\right)-\psi\left(r^{\prime}\right)\right\} d y^{\prime} d z^{\prime} \text {. }
$$

Now suppose that we always make

$$
\frac{y}{b}=\frac{y^{\prime}}{b^{\prime}} \text { and } \frac{z}{c}=\frac{z^{\prime}}{e^{\prime}} \text {; }
$$

then we have by the preliminary proposition

and we have also

$$
r_{1}=r_{1}^{\prime} \text { and } r_{2}=r_{3}^{\prime} \text {; }
$$

$$
\frac{d y d z}{d y d z}=\frac{b c}{b c} \text {. }
$$

Hence the first resolved attraction is to the sccond as be is to $b^{\prime} c$ '; and this establishes the theorem. 
It will be seen that the demonstration establishes something more than Ivory's theorem enunciates, namcly the following: take any elementary prism of the first ellipsoid the edges of which are chords parallel to an axis, and take the corresponding elementary prism of the second ellipsoid; then the attractions of these prisms resolved parallel to the axis on the corresponding points are as the products of the other axes: and Ivory's theorem follows from the fact that the ellipsoids may be supposed to be formed of corresponding elementary prisms.

We observe that one of these ellipsoids lies entirely within the other. For if not the points at which they intersect would lie on the curre of which the equations are

$$
\frac{x^{2}}{a^{2}}+\frac{y^{2}}{b^{2}}+\frac{z^{2}}{c^{2}}=1 \text {, and } \frac{x^{2}}{a^{12}}+\frac{y^{2}}{b^{\prime 2}}+\frac{z^{2}}{c^{12}}=1 ;
$$

the co-ordinates of the points of intersection must therefore satisfy the equation

$$
x^{2}\left(\frac{1}{a^{2}}-\frac{1}{a^{12}}\right)+y^{2}\left(\frac{1}{b^{2}}-\frac{1}{b^{12}}\right)+z^{2}\left(\frac{1}{c^{2}}-\frac{1}{c^{12}}\right)=0 .
$$

Since the ellipsoids are confocal this becomes

$$
\frac{x^{2}}{a^{2} a^{12}}+\frac{y^{2}}{b^{2} b^{\prime 2}}+\frac{z^{2}}{c^{2} c^{12}}=0 ;
$$

and this equation can only be satisfied by supposing $x, y$, and $z$ to vanish; and these values do not satisfy the equations to the ellipsoids. Thus the ellipsoids do not intersect at any point.

Hence to find the attraction of an ellipsoid of which the semi-axes are $a, b, c$ on an external particle of which the coordinates are $f^{\prime}, g^{\prime}, h^{\prime}$, we must first calculate the attraction, resolved parallel to the axes, of an ellipsoid of which the semi-axes are $a^{\prime}, b^{\prime}, c^{\prime}$ on an internal particle of which the co-ordinates are $f, g, h$; these six quantities being determined by the equations

$$
a^{12}-b^{12}=a^{2}-b^{2}, \quad a^{\prime 2}-c^{\prime 2}=a^{2}-c^{3},
$$




$$
\begin{gathered}
\frac{f^{\prime}}{a^{2}}+\frac{g^{2}}{b^{3}}+\frac{h^{\circ}}{c^{\prime}}=1, \\
j=\frac{a f^{\circ}}{a^{\prime}}, \quad g=\frac{b j^{\prime}}{b^{\prime}}, \quad h=\frac{c h^{\circ}}{c^{\prime}}
\end{gathered}
$$

and then the regolved parts of the required attraction will be these three calculated results, multiplied respectively by

$$
\frac{b c}{b c}, \frac{a b}{c a}, \frac{a b}{a b} \text {. }
$$

It may be shewn that there is only one ellipsoid which can have its semi-axes $a^{\prime}, b^{\prime}, e^{\prime}$ gatisfying the conditions required in Ivory's theorem.

Suppose that $a, b, c$ are in descending order of magnitude. Put $t$ for $c^{\prime 2}$; let $a^{2}-c^{2}=p$, and $l^{2}-c^{2}=q$, so that $p$ and $q$ are positive quantities. We have then

$$
a^{2}=p+t, \quad b^{2}=q+\ell ;
$$

thus we obtrin the following equation for determining $C$,

$$
\frac{f^{m}}{p+t}+\frac{g^{m}}{q+t}+\frac{h^{m}}{t}-1=0 .
$$

By examining the changes of sign of the expression which forms the lefi-hand member of this equation, we see that there is a root between $-p$ and $-q$, a root between $-q$ and 0 , and a root between 0 and $\infty$. Corresponding to the first root we should obtain an hyperbolnid of two sheots; curresponding to the second root an hyperboloid of one sheet; and corresponding to the third root an ellipsoid.

231. To prove that the resultant attruction of the particles of a body of any figure on a particle of achich tha distance is very great in comparison woth the gratest diameler of the attructing body, is evry nearly the same, as if the particles acere condensed at their centre of grarity and attracted accorcling to the same lase, uchatever that lave he.

Int the origin of co-ordinates be tnken at the centre of gravity of the attracting body, the axis of $x$ through the 
attracted particle; let $c$ be its abscissa, and $x, y, z$ the coordinates of any particle of the body, $\rho$ the density of that particle.

Then the distance between these two particles, or $r$,

$$
=\sqrt{ }\left\{(c-x)^{2}+y^{2}+z^{2}\right\} \text {. }
$$

Iet $r \phi\left(r^{2}\right)$ be the law of attraction; then the whole attraction parallel to the axis of $x$

$$
=\iiint \rho(c-x) \phi\left(c^{2}-2 c x+x^{2}+y^{2}+z^{2}\right) d x d y d z,
$$

the limits being obtained from the equation to the surface of the body. This attraction therefore

$$
=\iiint \rho(c-x)\left\{\phi\left(c^{2}\right)-\left(2 c x-x^{2}-y^{2}-z^{2}\right) \phi^{\prime}\left(c^{2}\right)+\ldots\right\} d x d y d z
$$

$=c \phi\left(c^{2}\right) \iiint \rho\left\{1-\frac{x}{c}\left(1+\frac{2 c^{2} \phi^{\prime}\left(c^{2}\right)}{\phi\left(c^{2}\right)}\right)+\left(y^{2}+z^{2}+3 x^{2}\right) \frac{\phi^{\prime}\left(c^{2}\right)}{\phi\left(c^{2}\right)}+\ldots\right\} d x d y d z$

$=M c \phi\left(c^{2}\right)+c^{2} \phi^{\prime}\left(c^{2}\right) \iiint \rho \frac{y^{2}+z^{2}+3 x^{2}}{c^{2}} d x d y d z+\ldots \ldots \ldots(A)$,

$M$ being the mass of the body, and $\iiint \rho x d x d y d z=0$, since $x$ is measured from the centre of gravity of the body.

Now suppose $x, y, z$ to be exceedingly small in comparison with $c$; then all the terms of $(A)$ after the first are extremely small in comparison with that term, it being observed that $c^{2} \phi^{\prime}\left(c^{2}\right)$ is of the same order as $c \phi\left(c^{2}\right)$ in terms of $c$. Hence the resultant attraction is very nearly $M c \phi\left(c^{2}\right)$; that is, it is very nearly the same as if the particles were condensed at their centre of gravity and attracted according to the law determined by the function $r \phi\left(r^{2}\right)$.

232. From Art. 224, it appears that when the law of attraction is that of the inverse square of the distance, a sphere composed of shells, each of which is homogeneous, attracts an external particle with a resultant force, which is the same as if the sphere were condensed at its centre. It may be shewn also that two such spheres attract each other 
in the same mannor as if each were condenued at ita centre. For consiler any element of masa forming jart of the firat spluere; the attraction of this on the second spluere will bo eyual and opprosite to the resultant attraction of the movond ophere on it, and will sherefure be the same as if the second sphere were collected at its centre. Similarly, the attraction of any other element of the first mpluere on the socomil will be the same as if the secomd were collected at its centre. Proceoding thus, we find that the whole action of the first spliere on the seound is the same as if the seovod were collecteol at its centre, and therefore the mutual attraction of the splueres is the same as if each were collected at its centre.

If the law of attraction be that of the direct distanoe, then two bolies of any shape attract each other with a resultant force which is the same as if each were collected at its centre of Eravity.

We proeed to general formula for the attraction of bodies of any form.

233. Iet there be a body of any form; let $\rho$ represent the density of an element, the volume of which is dxdydz, x, $y$, : being the co-ordinates of the element. Suppose the attraction between the particles of masseg $m$ and $m$ respectively, at a distance $r$, to be $m m^{\prime} F(r)$; then the components $X, Y, Z$ parallel to the axes, and from the origin, of the attraction of the body on a particle whose mass is unity, and co-ordinates $a, b, c$ are found by the equations

$$
\begin{aligned}
& X=\iiint \rho \frac{x-a}{r} F(r) d x d y d z, \\
& Y=\iiint \rho \frac{y-b}{r} F(r) d x d y d z, \\
& Z=\iiint \rho \frac{z-c}{r} F(r) d x d y d z,
\end{aligned}
$$

$$
r \text { being }=\left\{(x-a)^{2}+(y-b)^{2}+(z-c)^{2}\right\}
$$

The integrations are to be taken so as to iuclude all the elements of the attracting body. 
Let $\phi(r)$ be such a function of $r$ that $F(r)$ is its differential coeflicient with respect to $r$, and let

$$
U=\iiint \rho \phi(r) d x d y d z,
$$

the integrations being extended so as to include all the elements of the attracting body; then will

$$
\begin{aligned}
& \qquad \begin{aligned}
X=-\frac{d U}{d a}, \quad Y=-\frac{d U}{d b}, Z=-\frac{d U}{d c} \\
\text { For } \frac{d \phi(r)}{d a}=\frac{d \phi(r)}{d r} \frac{d r}{d a}=F(r) \frac{d r}{d a}=-F(r) \frac{x-a}{r}
\end{aligned} \\
& \qquad \begin{aligned}
X & =-\iiint \rho \frac{d \phi(r)}{d a} d x d y d z \\
& =-\frac{d}{d a} \iiint \rho \phi(r) d x d y d z \\
& =-\frac{d U}{d a} .
\end{aligned}
\end{aligned}
$$

therefore

Similarly, the equations $Y=-\frac{d U}{d b}$ and $Z=-\frac{d U}{d c}$ may be established.

It may be observed that if in any case, for example that of an infinite solid, the integral $U$ becomes infinite, but the differential coefficients $\frac{d U}{d a}, \frac{d U}{d b}, \frac{d U}{d c}$ are finite, the preceding values of $X, Y, Z$ will still be correct.

For suppose we take a finite portion of the solid; the components of its attraction will have for values the differential coefficients of $U$. Suppose now that we extend without limit the portion of the mass considered, the components of the attraction will always be

$$
-\frac{d U}{d e},-\frac{d U}{d b},-\frac{d U}{d c},
$$

whether $U$ increase without limit or not. Hence, if these three expressions tend to limits, those limits will be the com- 
ponents of the attraction of the infinite solid. And if they increase indefinitely, we may conclude that the attraction increases withent limit as the portion of the body cunnidered increases; this we expreas by saying that the attraction of the solid is infinite.

234. If the law of attraction be that of the inverse sguare, we have

$$
F(r)=\frac{1}{p}, \text { and } \phi(r)=-\frac{1}{r} .
$$

Let $V=-U$, that is, let

$$
V=\iiint \frac{p d x d y d z}{r} \ldots . . . \ldots \ldots \ldots \ldots . . . . . .(1) \text {; }
$$

then, as in the preceding Article, we have for the attractions parallel to the axes of $x, y, z$ respectively, and from the origin,

$$
X=\frac{d V}{d u}, \quad Y=\frac{d V}{d b}, \quad Z=\frac{d V}{d c} .
$$

The equation which gives $V$ is equiralent to the following operation:-decompese the attracting mess into indrfinitely small elements, and diride the mass of each elemeni by the distunce of that element from the attracted particle; the sum of these quetients is $V$. Hence, the value of $V$ will be quite independent of the axes, rectangular or polar, which we may find it convenient to employ. Suppose we use the ordinary polar formula, and take the position of the attracted particle fur the origin; then the element of volume is (Art. 130) $r^{2} \sin \theta \delta \phi \delta \theta \delta r$; therefore

$$
V=\iint f \rho r \sin \theta d \phi d \theta d r \ldots \ldots \ldots \ldots \ldots \text { (2). }
$$

Suppose the attractel particle forms part of the attracting mass ; then, sinee $r$ vanishes for those particles of the attracting mass which are in contact with the attracted particle, from equation (1) it would be doubrul if $V$ is finite in this case; but from (2) we abe that it really is finite.

235. To express by means of $V$ the altraction resolved along any line.

Let $s$ be the length of the are of any curve measured from a fixed point up to $P$ the attracted particle; $l, m$, $n$ the dirce- 
tion cosines of the tangent to this line at $P ; R$ the attraction resolved along this tangent; then

$$
\begin{aligned}
R & =l X+m Y+n Z \\
& =l \frac{d V}{d a}+m \frac{d V}{d b}+n \frac{d V}{d c} .
\end{aligned}
$$

Now, if we restrict ourselves to points lying on the line 8 , $V$ will become a function of $s$ alone; for $V$ is a function of $a, b$, and $c$, and each of these may be regarded as a function of $s$; thus we shall have by the differential calculus,

$$
\frac{d V}{d s}=\frac{d V}{d a} \frac{d a}{d s}+\frac{d V}{d b} \frac{d b}{d s}+\frac{d V}{d c} \frac{d c}{d s}
$$

and since $\frac{d a}{d s}=l, \frac{d b}{d s}=m, \frac{d c}{d s}=n$, we get

$$
R=\frac{d V}{d s} .
$$

\section{To examine the meaning of the function $V$.}

This function is of so much importance that it will be well to divell a little on its meaning.

In the first place it may be observed that the equation (1) contains a physical definition of $V$, which has nothing to do with the system of co-ordinates, rectangular, polar, or any other, which may be used to define algebraically the positions of $P$ and of the attracting particles. Thus $V$ is to be contemplated as a function of the position of $P$ in space, if such an expression may be allowed, rather than as a function of the-co-ordinates of $P$; although, in consequence of its depending upon the position of $P, V$ will be a function of the co-ordinates of $P$, of whatever kind they may be.

Secondly, it may be remarked that although an attracted particle has hitherto been conceived as situated at $P$, yet $V$ has a definite meaning depending upon the position of the point $P$, whether any attracted matter exist there or not. Thus $V$ is to be contemplated as having a definite value at each point of space, irrespective of the attracted matter which may exist at some places.

The function $V$ is called the potential of the attracting mass. 
237. To ealculate the value of $Y$ in the cune of a ophericul shell, the density being a function of the distunce frons the centre.

Take for the axis of $x$ the straight line joining the centre of the sphere with the attracted particle $I$, which is obviously tho direction of the resuleant attraction; let a be the distance of $P$ from the centre; uthe distance of any point in the attracting shell from the centre; $\theta$ and $\phi$ the other polar co-ordinates of this point; then the mass of the elensent at this point is $\rho u^{3} \sin \theta \delta u \delta \delta \delta$, and

$$
V=\int_{\omega_{0}}^{m_{0}} \int_{0}^{\infty} \int_{0}^{-\theta} \frac{\rho u^{2} \sin \theta d u d \theta d \phi}{r},
$$

where $u_{3}$ and $u_{2}$ are the internal and external radii of the shell; hence,

$$
V=2 \pi \int_{\omega_{1}}^{\omega_{0}} \int_{0}^{n} \frac{\rho u^{2} \sin \theta d u d \theta}{r} .
$$

Now

$$
r^{2}=u^{3}-2 a u \cos \theta+a^{2} \text {; }
$$

therefore

$$
\sin \theta \frac{d \theta}{d r}=\frac{r}{\omega u} \text {, }
$$

and

$$
V=\frac{2 \pi}{a} \iint \rho u d u d r \text {. }
$$

We must now distinguish three cases.

I. When $P$ is beyond the external surface, the limits of $r$ are $a-u$ and $a+u ;$ therefore

$$
\begin{aligned}
& V=\frac{2 \pi}{a} \int_{\omega}^{\omega} \int_{a-u}^{a+\infty} \rho u d u d r \\
& =\frac{4 \pi}{a} \int_{-i s}^{\omega_{0}} \rho v^{3} d u
\end{aligned}
$$

But if $M$ denote the mass of the Epherical shell,

$$
M=1 \pi \int_{n}^{\omega_{s}} \rho x^{2} d u
$$


therefore

$$
V=\frac{M}{a} \text {. }
$$

Hence, $X=\frac{d T}{d a}=-\frac{M}{a^{3}}$, or the attraction is the same as if the mass of the shell were collected at its centre; this was proved in Art. 212.

II. When $P$ is within the internal surface, the limits of $r$ are $u-a$ and $u+a$; therefore

$$
\begin{aligned}
V & =\frac{2 \pi}{a} \int_{u_{1}}^{u_{3}} \int_{u-a}^{u+a} \rho u d u d r \\
& =4 \pi \int_{u_{1}}^{v_{u}} \rho u d u \ldots \ldots \ldots . . . . . .
\end{aligned}
$$

Since this is independent of $a$, we have

$$
\frac{d V}{d a}=0 .
$$

This is equivalent to the result found in Art. 213.

III. By combining the results contained in equations (1) and (2), we see that if $P$ be between the bounding surfaces of the shell,

$$
V=\frac{4 \pi}{a} \int_{u_{1}}^{a} \rho u^{2} d u+4 \pi \int_{a}^{u_{s}} \rho u d u .
$$

From this we may deduce a result involved in Arts. 212 and 213 , namely, that the resultant attraction is the same as if all the matter which is nearer to the centre than $P$ were collected at the centre, and the rest of the matter neglected.

238. At any point $(a, b, c)$ where there is no particle of the attracting mass, the function $V$ satisfies the partial differential equation

$$
\frac{d^{2} V}{d a^{2}}+\frac{d^{2} V}{d l^{2}}+\frac{d^{2} V}{d c^{2}}=0
$$


For since $r=\left\{(x-a)^{3}+(y-b)^{2}+(z-c)^{2}\right\}$,

$$
\begin{aligned}
& \frac{d}{d a}\left(\frac{1}{r}\right)=\frac{x-a}{r^{2}}, \frac{d}{d b}\left(\frac{1}{r}\right)=\frac{y-b}{r^{2}}, \frac{d}{d c}\left(\frac{1}{r}\right)=\frac{g-c}{r^{2}}, \\
& \frac{d^{3}}{d a^{3}}\left(\frac{1}{r}\right)=\frac{3(x-a)^{2}}{r^{2}}-\frac{1}{r^{3}} \text {, } \\
& \frac{d^{3}}{d b^{3}}\left(\frac{1}{r}\right)=\frac{3(y-b)^{0}}{r^{2}}-\frac{1}{r^{3}} \\
& \frac{d}{d c^{3}}\left(\frac{1}{r}\right)=\frac{3(z-c)^{2}}{r^{2}}-\frac{1}{p^{3}} \text {; }
\end{aligned}
$$

therefore

$$
\frac{d^{0}}{d a^{2}}\left(\frac{1}{r}\right)+\frac{d^{2}}{d b^{2}}\left(\frac{1}{r}\right)+\frac{d^{2}}{d c^{2}}\left(\frac{1}{r}\right)=0 \text {. }
$$

Now

$$
V=\iiint \frac{\rho d x d y d z}{r} ;
$$

therefore

$$
\frac{d^{2} V}{d a^{3}}=\iiint \frac{d^{2}}{d a^{2}}\left(\frac{1}{r}\right) \rho d x d y d z \text {, }
$$

and similar expressions hold for $\frac{d^{2} V}{d b^{2}}$ and $\frac{d^{2} V}{d c^{2}}$; therefure

$$
\frac{d^{2} V}{d a^{2}}+\frac{d^{2} V}{d b^{2}}+\frac{d^{2} V}{d c^{2}}=0
$$

This result holds so long as the attracted particle is not in contact with the attracting mass. If, however, the attracted particle is in contact with the attracting mass, $r$ can vanish, and therefore $\frac{1}{r}$ and its differential cocfficients become infinile; the preceding demonstration does not hold in this case.

239. At an internal point $(a, b, c)$ about which the density is $\rho$, the function $V$ satiagies the equation

$$
\frac{d^{2} V}{d \omega^{3}}+\frac{d^{2} V}{d b^{*}}+\frac{d^{2} V}{d \theta^{2}}=-4 \pi \rho
$$

To determine the value of $\frac{d^{2} V}{d a^{3}}+\frac{d^{2} V}{d b^{2}}+\frac{d^{2} V}{d c^{2}}$ in this case, พ. 8. 
suppose a sphere described in the body so that it shall include the attracted particle, and let $V=V_{3}+V_{3}$, where $V_{2}$ refers to the sphere and $V_{1}$ to the remainder of the attracting body; then

$$
\begin{aligned}
\frac{d^{2} V}{d a^{8}}+\frac{d^{2} V}{d b^{2}}+\frac{d^{2} V}{d c^{8}} & =\frac{d^{3} V_{3}}{d a^{3}}+\frac{d^{2} V_{1}}{d b^{3}}+\frac{d^{2} V_{3}}{d c^{2}} \\
& +\frac{d^{3} V_{8}}{d a^{3}}+\frac{d^{3} V_{8}}{d b^{8}}+\frac{d^{2} V_{8}}{d c^{8}} \\
& =\frac{d^{2} V_{3}}{d a^{2}}+\frac{d^{2} V_{3}}{d b^{3}}+\frac{d^{3} V_{3}}{d c^{3}},
\end{aligned}
$$

by what has been already proved.

Now the centre of the sphere may be chosen as near the attracted particle as we please, and the radius of the sphere may be taken so small that its density may be considered ultimately uniform, and equal to that at the point $(a, b, c)$.

Let $\alpha, \beta, \gamma$ be the co-ordinates of the centre of the sphere; then the attractions of the sphere on the particle parallel to the axes are, by Art. 212,

$$
\frac{4}{3} \pi \rho(a-a), \frac{4 \pi \rho}{3}(b-\beta), \frac{4 \pi \rho}{3}(c-\gamma) ;
$$

therefore $\frac{d V_{2}}{d a}=-\frac{4 \pi \rho}{3}(a-\alpha), \frac{d^{2} V}{d a^{2}}=-\frac{4 \pi \rho}{3}$,

$$
\begin{aligned}
& \frac{d V_{8}}{d b}=-\frac{4 \pi \rho}{3}(b-\beta), \frac{d^{2} V_{3}}{d b^{2}}=-\frac{4 \pi \rho}{3}, \\
& \frac{d V_{3}}{d c}=-\frac{4 \pi \rho}{3}(c-\gamma), \frac{d^{2} V_{3}}{d c^{2}}=-\frac{4 \pi \rho}{3} ;
\end{aligned}
$$

therefore

$$
\frac{d^{2} V_{2}}{d a^{3}}+\frac{d^{2} V_{3}}{d b^{2}}+\frac{d^{2} V_{8}}{d c^{3}}=-4 \pi \rho ;
$$

therefore

$$
\frac{d^{2} V}{d a^{2}}+\frac{d^{2} V}{d b^{2}}+\frac{d^{2} V}{d c^{2}}=-4 \pi \rho
$$

240. Application to the Sphere. In Art. 237 we hav calculated $V$ by direct integration in the case of a body com 
posed of homogeneous spherieal shells. We may also dedues its value by means of the equations in Art. 235 and 239. This we shiall now do. If a sphere be composed of homogeneous shells, $V$ will be a function of the distance $r$ of the centre of the spliere from the attracted particle; the resultant attraction will act along the straight line which joins these two points, and will be denoted by $\frac{d V}{d r}$.

The equation

$$
r^{2}=a^{2}+b^{2}+c^{2}
$$

will give $\quad \frac{d r}{d a}=\frac{a}{r}, \frac{d r}{d b}=\frac{b}{r}, \frac{d r}{d c}=\frac{c}{r}$;

hence

$$
\frac{d V}{d a}-\frac{d V}{d r} \frac{d r}{d a}=\frac{d V}{d r} \frac{a}{r} \text {; }
$$

therefore

$$
\frac{d^{2} V}{d a^{3}}=\frac{a^{2}}{r^{3}} \frac{d^{2} V}{d r^{3}}+\frac{1}{r} \frac{d V}{d r}-\frac{a^{3} \frac{d V}{P^{2}}}{d r} ;
$$

similarly

$$
\frac{d^{2} V}{d b^{3}}=\frac{b^{3}}{r^{2}} \frac{d^{3} V}{d r^{3}}+\frac{1}{r} \frac{d V}{d r}-\frac{b^{2} d V}{d r} \text {. }
$$

and

$$
\frac{r^{2} V}{d c^{3}}=\frac{e^{2}}{r^{2}} \frac{d V}{d r^{3}}+\frac{1}{r} \frac{d V}{d r}-\frac{c^{2}}{r^{3}} \frac{d V}{d r} \text {. }
$$

By alding these equations we have, by Art. 238, at a point where there is no particle of the attracting mass,

$$
\frac{d^{2} V}{d r^{3}}+\frac{2 d r}{r} \frac{d r}{d v}=0
$$

This may be written

therefore

$$
\begin{aligned}
& \frac{d}{d r}\left\{r^{a} \frac{d V}{d r}\right\}=0 ; \\
& \frac{d V}{d r}=\frac{C}{r^{2}},
\end{aligned}
$$

where $C$ is some constant.

Suppose the sphere to be hollow, and that the attracted purticle is within the inner surface, the malius of which we 
shall denote by $\gamma_{1}$. Since the attraction ought evidently to vanish when $r=0$, we must have $C=0$; therefore $\frac{d V}{d r}=0$. Hence the attraction always vanishes, and the particle is in equilibrium whatever be its position within the unoccupied part of the sphere.

Suppose next that the particle forms part of the mass of the sphere; we have, by Art. 239,

$$
\frac{d^{2} V}{d r^{2}}+\frac{2}{r} \frac{d V}{d r}=-4 \pi \rho
$$

$\rho$ being a given function of $r$.

Multiply by $r^{2}$, and integrate from the value $r_{1}$ of $r$; since $\frac{d V}{d r}=0$ for all points in the interior, it is so at the limit $r_{1}$;

thus

$$
r^{2} \frac{d V}{d r}=-4 \pi \int_{r_{1}}^{r} \rho r^{2} d r .
$$

But $\int_{r_{1}}^{r} 4 \pi r^{2} \rho d r$ is the mass comprised within that surface of the sphere which passes through the attracted particle. If we call it $M^{\prime}$, we have

$$
\frac{d V}{d r}=-\frac{M^{\prime}}{r^{3}} .
$$

The absolute value of the attraction will therefore be $\frac{M^{\prime}}{r^{2}}$; it is the same as if the mass $M I^{\prime}$ acted alone and were collected at its centre.

If the attracted particle is on the exterior surface having its radius $=r_{2}$, we have, if $M$ be the whole mass of the hollow sphere,

$$
\frac{d V}{d r}=-\frac{M}{r_{2}^{2}},
$$

and the attraction exercised upon this particle will have for its value

$$
\frac{M}{r_{3}^{2}} \text {. }
$$


Lastly, conshiler a particle outaile the sphere; that is, for which $r$ is greater than $r_{8}$; we have, as in the firat case,

$$
\frac{d V}{d r}=\frac{C}{r} \text {. }
$$

But in consequence of the discontinuity arising from thas particles of the mass, the constant $C$ is not restricted to have the same value as for the interior points. To determine it we put $r=r_{2}$; then, from the preceding casse, we whight to have

$$
\frac{d V}{d r}=-\frac{M}{r_{0}} \text {. }
$$

therefore

$$
C=-M ;
$$

and we shall have for external points,

$$
\frac{d V}{d r}=-\frac{M}{r} \text {. }
$$

The attraction will therefore have for its value

$$
\frac{M}{r}
$$

\section{This agrees with Art. 212.}

The preceding application to the sphere serves very well to illustrate the formula, but it does not give an independent demonstration of the results which it involves; because the process in Art. 239 assumes that the attraction of a ipphere on an internal particle is known. But we may easily ottain the fiucts connected with the attraction of a spherical shell without using Art. 239.

Consider a splerical shell where the density is any function of the radius; then we have, as shewn at the commencement of the present Article, the result

$$
\frac{d V}{d r}=\frac{C}{r} \text {, }
$$

where $C$ is onnstant when we pross from point to point withous entering the attracting mass. 
For any point within the inner surface of the shell $C=0$, because the attraction must vanish when $r=0$.

For any point without the outer surface of the shell $C=-M$, because for points at an indefinitely great distance the resultant attraction of the shell must be the same as if the shell were condensed at its centre of gravity; see Art. 231.

Thus the required results are obtained.

241. Aprlication to an indefinite cylinder. Consider next a hollow indefinite cylinder composed of homogeneous shells, the density being a function of the distance from the axis of the cylinder which we take for the axis of $z$. Its action upon any particle will be directed towards the point where the axis is cut by a perpendicular plane passing through the attracted particle. Take this point of the axis for origin; let $r$ be its distance from the attracted particle; the attraction will depend only on $r$, and its value will be

$$
\frac{d V}{d r} .
$$

But for the points which are not part of the mass of the cylinder, we have, by Art. 238, observing that $V$ is independent of $c$,

whence

$$
\frac{d^{2} V}{d a^{2}}+\frac{d^{2} V}{d b^{2}}=0
$$

$$
\frac{d^{2} V}{d r^{2}}+\frac{1}{r} \frac{d V}{d r}=0
$$

Multiplying by $r$, we have

therefore

$$
\frac{d}{d r}\left(r \frac{d V}{d r}\right)=0
$$

$$
\frac{d V}{d r}=\frac{C}{r},
$$

$C$ being some constant.

We observe, as in the case of a hollow sphere, that the points exterior to the cylindrical shell and those in the interior being separated by those of the shell, for which the circum- 
stanees are different, there is a discontinuity in passing from values of $r$ greater than the radius of the external surface, to those of $r$ less than the radius of the internal surface.

For points of the interior of the shell $\mathrm{C}$ is invarialile; but it is obviously $=0$ when $r=0$; therefure for all prints in the interior

$$
\frac{d V}{d r}=0
$$

Hence we conclude, that an indefinite hollow cylinder composed of homgenenus shells excreises no altraction on a point situated within the interior of its internal surfuce.

Let us now find the value of $\frac{d V}{d r}$ for points belonging to the mass of the cylinder; for these puints we have, by Art. 239,

$$
\frac{d^{2} V}{d r^{T}}+\frac{1}{r} \frac{d V}{d r}=-4 \pi \rho,
$$

and we find by integration, calling $r$, the radius of the internal surface,

$$
r \frac{d V}{d r}=-4 \pi \int_{r_{1}}^{r} \rho r d r
$$

No constant is necessary, because $\frac{d V}{d r}=0$ when $r=r_{1}$, since it is so fir all the points of the interior of the surface of which the ralius is $r_{8}$. Put $r=r_{2}$, then

$$
\frac{d V}{d r}=-\frac{4 \pi}{r_{2}} \int_{n}^{r} \rho r d r .
$$

For external points we ought to have

$$
\frac{d V}{d r}=\frac{C}{r} \text {. }
$$

Iake $r=r_{3}$, then, by reasun of the preceding eqquation,

$$
C=-4 \pi \int_{n}^{n} \text { prdr. }
$$


The constant being thus determined, we have for all values of $r$ greater than $r_{s}$,

$$
\frac{d V}{d r}=\frac{C}{r},
$$

and the attraction of the cylinder will be

$$
\frac{C}{r} \text {. }
$$

We shall now give some propositions extracted from an article by Professor Stokes, in the fourth volume of the Cambridge and Dublin Mathematical Journal, to which we have been already indebted in Art. 236.

242. A surface of equilibrium is one on which a particle would rest in equilibrium if acted on by the forces of the system, the surface being supposed fixed.

If $V$ be the potential of an attracting body on a particle, then $V=$ constant, is the equation to a surface of equilibrium with respect to the attraction of the body. For we have shewn in Art. 235, that $\frac{d V}{d s}$ is equal to the attraction resolved along the tangent to a curve drawn through the attracted particle, but if this curve be on the surface $V=$ constant, then $\frac{d V}{d s}=0$; that is, there is no force acting on $P$ in the direction of any tangent to the surface $V=$ constant. Hence, if $P$ be placed on the surface, it will remain in equilibrium. (Art. 33.)

Lines of force are curves traced so that the tangent at any point is the direction of the resultant force at that point. Hence the lines of force are perpendicular to the surfaces of equilibrium.

243. If $S$ be any closed surface to which all the attracting mass is external, $d S$ an element of $S$, and $d n$ an element of the normal drawn outwards at $d S$, then

$$
\int \frac{d V}{d n} d S=0,
$$

the integral being taken throughout the whole surface $S$. 
Let $m$ ' be the mass of any attracting particle which in situated at the point $P, P$ being by hypothesis external to $S$. Through $P$ draw any right line $L$ cutung $S$, and produce is indefinitely in one direction fron $P$. The line $L$ will in general cut $S$ in two points; but if the surface $S$ be recentrane (that is, a closed surface which may be cut by a tangent plane), it may eut it in four, six, or any even number of points. Denote the points of section, taken in order, by $P_{1}, P_{3}, P_{0}$. die, $P$, being that which lies nearest to $P$. With $P$ for vertex, describe about the line $L$ a conical surface containing an infinitely small solid angle measured by the area $a$ which the conical surface cuts out from a sphere of radius unity, with the vertex of the cone as its centre; and denote by $A, A, \ldots$ the areas which the conical surface cuts out from $\mathcal{B}$ about the points $P_{1}, P_{2}, \ldots$. . Let $\theta_{1}, \theta_{3}$, be the angles which the normals drawn outwards at $F_{1}, P_{8}, \ldots \ldots$ make with the line $L_{4}$ taken in the direction from $P_{1}$ to $P_{3}, N_{1}, N_{1}, \ldots \ldots$ the attractions of $m^{\prime}$ at $P_{1}, P_{s}, \ldots \ldots$ resolved along the normals; $r_{1}$, $r_{3}, \ldots \ldots$ the distances of $P_{1}, P_{3}, \ldots \ldots$ from $P^{P}$. It is evident that the angles $\theta_{1}, \theta_{2}, \ldots \ldots$ will be alternately acute and obtuse. Then we have

$$
N_{1}=\frac{m^{\prime}}{r_{1}^{2}} \cos \theta_{2}, \quad N_{2}=-\frac{m^{\prime}}{r_{2}^{\prime}} \cos \left(\pi-\theta_{2}\right), \& c .
$$

We have also in the limit,

$$
A_{1}=\alpha r_{2}{ }^{2} \sec \theta_{2}, \quad A_{3}=\alpha r_{2}^{2} \sec \left(\pi-\theta_{2}\right), \text { Sic. ; }
$$

and therefore

$$
N_{1} A_{1}=a m^{\prime}, \quad N_{3} A_{2}=-\alpha m^{\prime}, \quad N_{3} A_{2}=a m^{\prime}, \text { Scc ; }
$$

and therefore, since the number of points $P_{v}, P_{g} \ldots$ is even,

$N_{1} A_{1}+N_{2} A_{2}+N_{3} A_{3}+N_{1} A_{4} \ldots=a m^{\prime}-a m^{\prime}+a m^{\prime}-a m^{\prime} \ldots=0$.

Now the whole solid angle contained within a conical surface described with $P^{\nu}$ fur vertex, so ns to circumscribe $S$, may be divided into an infinite number of elementary solid angles, to each of which the preceding resoning will apply; and it is evident that the whole surface $S$ will thus be exhausted. Wo have, therefore,

$$
\text { limit of } \mathbf{S A}=0 \text {; }
$$


or, by the definition of an integral,

$$
\int N d S=0 .
$$

The same will be true of each attracting particle $m^{\prime}$; and therefore, if $N$ refer to the attraction of the whole attracting mass, we shall still have $\int N d S=0$. But, by Art. $235, N=\frac{d V}{d n}$, which proves the proposition.

244. If $V$ be the potential of any mass $M_{1}$, and if $M_{0}$ be the portion of $M_{1}$ contained within a closed surface $S$, then

$$
\int \frac{d V}{d n} d S=-4 \pi M_{0}
$$

$d n$ and $d S$ having the same meaning as in Art. 243, and the integration being extended to the whole surface $S$.

Let $m^{\prime}$ be the mass of an attracting particle situated at the point $P^{\prime}$ inside $S$. Through $P$ draw a right line $L$, and produce it indefinitely in one direction. This line will in general cut $S$ in one point; but if $S$ be a re-entrant surface, it may be cut by $L$ in three, five, or any odd number of points. About $L$ describe a conical surface containing an infinitely small solid angle $\alpha$, and having its vertex at $P$, and let the rest of the notation be as in Art. 243. In this case, the angles $\theta_{1}, \theta_{8}, \ldots$ will be alternately obtuse and acute, and we shall have

$$
\begin{aligned}
& N_{1}=-\frac{m^{\prime}}{r_{2}{ }^{2}} \cos \left(\pi-\theta_{1}\right)=\frac{m^{\prime}}{r_{1}{ }^{2}} \cos \theta_{2}, \\
& A_{1}=\alpha r_{1}^{2} \sec \left(\pi-\theta_{1}\right)=-\alpha r_{1}^{2} \sec \theta_{1},
\end{aligned}
$$

and therefore

$$
N_{1} A_{1}=-\alpha m^{\prime} \text {. }
$$

Should there be more than one point of section, the terms $N_{9} A_{2}, N_{3} A_{3}$, \&c. will destroy each other two and two, as in Art. 243. Now all angular space round $P$ may be divided into an infinite number of solid angles such as $\alpha$, and it is evident that the whole surface $S$ will thus be exhausted. We get, therefore,

$$
\text { limit of } \Sigma N A=-\Sigma \alpha m^{\prime}=-m^{\prime} \Sigma x \text {; }
$$

or, since $\quad \Sigma \alpha=4 \pi, \int N d S=-4 \pi m^{\prime}$. 
The same formula will apply to any other internal par. ticle, and it has been shewn in Arr. 243, that for an extemal particle $(\mathrm{N} d S=0$. Hence, adding together all the rmalts, and taking $N$ now to refer to the attraction of all the particles, both internal and external, we get $\int A d S=-4 \pi M_{\text {. }}$ But $N=\frac{d V}{d n}$, which proves the proposition.

245. For the researches of M. Chasles on the attraction of ellipsoids, we refer to Duhamel's Courg de MCeanique, or to the original memoirs in the Jumrnal de TEoble Rolylechnigue, tom. $\mathrm{XV}$, and the Memeirea...des Savans Etrangers, tom. IX. In the original memoirs will be found copious references to preceding writers on the subject.

On the general theory of attractions, the student may consule a memoir by Canss, translated in Taglor's $S$-iemifie Memoirs, vol. IIt., and in Liouville's Juernal de Mathermatigues, fom. VII.; and also a memoir by M. Chasles in the Conmaisance des Tempa pour lannée is45.

Valuable notes by Plana on some of Newton's propositions respecting attractions will be found in the Memerie della lieale Acudemia...di Torino, second series, vol. X1., 1851.

Some further references will be seen in the article by Professor Stokes already cited.

For the application to the theory of electricity, we refer to a series of articles by Professor Thomson in different volumes of the Cambridge and Inablin Mathematical Journal. See vol. 1. p. 94, and vol. III. p. 140.

246. The following propositions will illustrate the subject of the present Chapiter.

I. To find the attraction of a uniform lamina in the form of a regular polygon on a particle situated in a straight line drawn through the centre of the lamina at right angles to its plune.

Iet $n$ he the number of sides in the polygon, $a$ the length of the perpendicular from the centre of the jolygon on a side. Lot axes of $x$ and $y$ be drawn through the centre of the 
polygon, the axis of $x$ being perpendicular to a side. Let $c$ be the distance of the particle from the lamina. The resultant attraction acts along the straight line which joins the particle with the centre of the lamina; and its value is

$$
\mu \iint \frac{c d x d y}{\left(c^{2}+x^{2}+y^{2}\right)^{2}} .
$$

The integration must extend over the area of the polygon. To effect the integration it is convenient to transform to polar co-ordinates; thus we obtain

$$
\mu c \iint \frac{r d r d \theta}{\left(c^{2}+r^{2}\right)^{\frac{2}{2}}} .
$$

We must integrate with respect to $r$ from $r=0$ to $r=a \sec \theta$, and then with respect to $\theta$ from $\theta=0$ to $\theta=\frac{\pi}{n}$; and multiply the result by $2 n$.

Now

$$
\int \frac{r d r}{\left(c^{2}+r^{2}\right)^{\frac{1}{1}}}=-\frac{1}{\left(c^{2}+r^{2}\right)^{d}}
$$

taking this between the limits we obtain

$$
\frac{1}{c}-\frac{\cos \theta}{\sqrt{c^{2} \cos ^{2} \theta+a^{3}}} \text {. }
$$

Hence the required result is

$$
2 n \mu c \int_{0}^{\pi}\left\{\frac{1}{c}-\frac{\cos \theta}{\sqrt{c^{2} \cos ^{8} \theta+a^{2}}}\right\} d \theta,
$$

that is

$$
2 \mu \pi-2 n \mu c \int_{0}^{\frac{\pi}{n}} \frac{\cos \theta d \theta}{\sqrt{\left(a^{2}+c^{3}-c^{2} \sin ^{2} \theta\right)}},
$$

that is

$$
2 \mu \pi-2 n \mu \sin ^{-1} \frac{c \sin \frac{\pi}{n}}{\sqrt{\left(a^{2}+c^{2}\right)}} .
$$


II. 'To find the attraction of a unifurm Intrina in the firm of a rectangle on a particle situated in a straight Tise Arawn through the centre of the lamina at right angles to its plane.

Iat $2 a$ and $2 b$ be the length and breadth of the rectangle. c the distance of the particle from the lassina. Proveeding as before we obtain the expreasion

$$
\mu c \iint \frac{r d r d \theta}{\left(c^{3}+r^{2}\right)} .
$$

We have to divide the integral into two parts. For one part we integrate with respect to $r$ from $r=0$ to $r=a$ ase $\theta$, and then with respect to $\theta$ from $\theta=0$ to $\theta=\tan ^{-1} \frac{b}{a}$. For the other part we integrate with respect to $r$ frum $r=0$ to $r=b \operatorname{cosec} \theta$, and then with respect to $\theta$ from $\theta=\tan ^{-6} \frac{b}{a}$ to $\theta=\frac{\pi}{2}$. We multiply the result by 4 .

$$
\int_{0}^{\infty \sec \theta} \frac{r d r}{\left(c^{3}+r^{2}\right)^{\prime}}=\frac{1}{c}-\frac{\cos \theta}{\sqrt{\left(c^{2} \cos ^{3} \theta+a^{5}\right)}} \text {. }
$$

Integrate with respect to $\theta$; thus we get

$$
\begin{gathered}
\frac{\theta}{c}-\frac{1}{c} \sin ^{-1} \frac{c \sin \theta}{\sqrt{\left(a^{3}+c^{2}\right)}} . \\
\int_{0}^{b \operatorname{cosec} \theta} \frac{r d r}{\left(c^{3}+r^{2}\right)^{t}}=\frac{1}{c}-\frac{\sin \theta}{\sqrt{\left(c^{3} \sin ^{3} \theta+b^{5}\right)}}
\end{gathered}
$$

Integrate with respect to $\theta$; thus we get

$$
\frac{\theta}{c}+\frac{1}{c} \sin ^{-3} \frac{c \cos \theta}{\sqrt{\left(b^{2}+\sigma^{2}\right)}} \text {. }
$$

Ilence the required result is

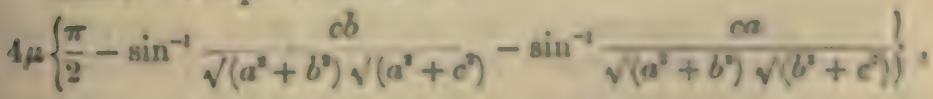

$$
\begin{aligned}
& \text { or } 4 \mu\left\{\cos ^{-1} \frac{d}{\sqrt{\left(a^{3}+b^{2}\right) \sqrt{\left(a^{2}+c^{2}\right)}}}-\sin ^{-3} \sqrt{\left(a^{2}+b^{2}\right) \sqrt{\left(b^{2}+c^{3}\right)}}\right\} \text {, } \\
& 4 \mu \sin ^{-1} \frac{a b}{\sqrt{\left(a^{3}+c^{3}\right) \sqrt{\left(b^{2}+c^{5}\right)}}} \text {. }
\end{aligned}
$$


III. Required the form of a homogeneous solid of revolution of given volume, which shall exercise the greatest attraction in a given direction on a given particle, the attraction varying as any inverse power of the distance.

Take the given particle as the origin, and the given direction as the straight line from which to measure angular distance; let $r, \theta$ be the polar co-ordinates of any point in a fixed plane passing through the given direction. Then if the attraction vary inversely as the $n^{\text {th }}$ power of the distance, the attraction of an element whose co-ordinates are $r$ and $\theta$ may be denoted by $\frac{\mu}{r^{n}}$; and the resolved part of this attraction in the giren direction will be $\frac{\mu}{r^{\mu}} \cos \theta$. Hence the equation

$$
\frac{\mu}{r^{m}} \cos \theta=\text { constant }
$$

represents a curve such that a given element placed at any point of it will exert the same attraction on the given particle along the given direction. Hence this equation will represent the curve which by revolving round the given direction will generate the required solid of greatest attraction, the constant being determined $\mathrm{so}$ as to give to the solid the prescribed volume. It is obvious that such is the case, because the surface we thus obtain separates space into two parts, and any element outside the surface exercises a less attraction along the given direction than it would if placed within the surface.

Some references connected with this problem will be found in the History of the... Calculus of Variations ..., page 485.

IV. Every element of the arc of a polar curve attracts with a force which varies inversely as the $n^{\text {th }}$ power of the distance: determine the form of the curve when the resultant attraction of any arc on a particle at the pole bisects the angle between the radii vectores of the extremities of the arc.

Take the pole as origin, and any straight line through it as the initial line. Let $r$ and $\theta$ be the polar co-ordinates of 
an clement de of the are. Resolve the attraction of the sare on a particle at the pole along the initial line, and at right angles to it; we obtain for these two compronents

$$
\int_{0}^{e} \frac{\mu}{r} \cos \theta \frac{d s}{d \theta} d \theta \text { and } \int_{0}^{\infty} \frac{\mu}{r} \sin \theta \frac{d s}{d \theta} d \theta \text {, }
$$

where the are considered extends from $\theta=0$ to $\theta=a$. Hence, by hypothesis,

$$
\frac{\int_{0}^{\theta} \frac{\mu}{r^{\mu}} \sin \theta \frac{d s}{d \theta} d \theta}{\int_{0}^{\rho} \frac{\mu}{r^{\mu}} \cos \theta \frac{d \theta}{d \theta} d \theta}=\tan \frac{\alpha}{2} .
$$

Put $\phi(\theta)$ for $\frac{1}{r^{n}} d \theta$; thus we have

$$
\int_{0}^{\infty} \phi(\theta) \sin \theta d \theta=\tan \frac{\alpha}{2} \int_{0}^{\infty} \phi(\theta) \cos \theta d \theta \text {. }
$$

Now this relation is to hold for all values of $\alpha$, and thervfore we may differentiate both sides with respect to a. Thus, by Integral Calculus, Chapter Ix., we have

$$
\phi(\alpha) \sin \alpha=\frac{1}{2} \sec ^{2} \frac{\alpha}{2} \int_{0}^{\varphi} \phi(\theta) \cos \theta d \theta+\tan \frac{\alpha}{2} \phi(\alpha) \cos \alpha ;
$$

therefore

$$
2 \phi(\alpha)\left(\sin \alpha-\tan \frac{\alpha}{2} \cos \alpha\right) \cos ^{\circ} \frac{\alpha}{2}=\int_{0}^{0} \phi(\theta) \cos \theta d \theta,
$$

that is

$$
\phi(\alpha) \sin \alpha=\int_{0}^{0} \phi(\theta) \cos \theta d \theta \text {. }
$$

Differentiate again with respect to $\propto$; thus

$$
\phi(x) \cos \alpha+\sin \alpha \frac{d}{d a} \phi(\alpha)=\phi(\alpha) \cos \alpha \text {; }
$$

therefore

$$
\frac{d}{d a} \phi(a)=0 \text {. }
$$


Thus $\phi(\alpha)$ is constant for all values of $\alpha$, that is

$$
\frac{1}{r^{\circ}} \frac{d s}{d \theta}=\text { a constant }=k \text { say. }
$$

This result might have been anticipated : it expresses that elements of the curve which subtend equal infinitesimal angles at the pole exert equal actions on the particle there.

Therefore

$$
r^{2}+\left(\frac{d r}{d \theta}\right)^{2}=k^{2} r^{2 n} ;
$$

this leads to either $\frac{d r}{d \theta}=0$, or else

$$
\left(\frac{d \theta}{d r}\right)^{2}=\frac{1}{k^{2} r^{2 n}-r^{2}} .
$$

The former supposition makes $r$ constant, and so gives a circle. Taking the latter, and putting $\frac{1}{u}$ for $r$ we have

$$
d \theta=\frac{u^{n-2} d u}{\sqrt{(}\left(l^{2}-u^{2 n-2}\right)},
$$

so that

$$
(n-1) \theta+C=\sin ^{-1} \frac{u^{n-1}}{k},
$$

where $C$ is a constant.

Therefore

$$
\frac{1}{r^{n-1}}=k \sin \{(n-1) \theta+C\} .
$$

If $n=2$ we obtain

$$
1=k r \sin (\theta+C),
$$

which is the equation to a straight line: see Art. 204.

If $n=3$ we obtain

$$
1=k r^{2} \sin (2 \theta+C),
$$

which is the equation to a rectangular hyperbola, the pole being at the centre. 


\section{EXAMTPLES.}

In the following Examples the ordinary law of attraction is to be assumed, unless the contrary be stated.

1. A solia is generated by the revolution of a sector of a circle about one of its bounding radii; find the attraction on a particle at the centre. Tiesule. $\quad$ rap sin' $\beta$.

2. The rim of a hemispherical bowl consists of matwer repelling with a force varying directly as the distance; shew that a particle will reat when placed anyelvere on the concave surface.

3. A tnbe in the form of a parabola is placed with its axis vertionl and vertex downwards; a heavy particle is placed in the tube, and a repulsive force acts aling the ordinate upon the particle: find the law of force that it may austain the particle in any position.

4. A portion of a cylinder of uniform density is bounded by a spherical surface, the radius of which is greater than that of the cylinder, and the centre coincides with the midalle point of the base; find the attraction on a particle at this point.

Result. $2 \pi \rho a-\frac{\pi \rho a^{2}}{b}$; where $a$ is the radius of the cylinder and $b$ the radius of the sphere.

5. Find the resultant attraction of a spherical segment on a particle at its vertex.

$$
\text { Result. } 2 \pi h p\left\{1-\frac{1}{3} \sqrt{ }\left(\frac{2 h}{a}\right)\right\} \text {. }
$$

where $a$ is the radius of the sphere and $h$ the beight of the segment.

6. Find the resultant attraction of a spherical segment on a particle at the centre of its bases

$$
\text { Resule. } \frac{2 \pi h \rho}{3(a-h)^{2}}\left\{\left(3 a^{2}-3 a h+h^{2}-(2 a-h)^{2} h\right\}\right) \text {. }
$$

T. S. 
7. Find the locus of a point such that its resultant attraction on a fixed straight line may always pass through a fixed point in the straight line.

Result. A sphere.

8. Find the attraction of a segment of a paraboloid of revolution, bounded by a plane perpendicular to its axis, on a particle at the focus.

Result. $4 \pi \rho a \log \frac{x+a}{a}$, where $x$ is the distance of the bounding plane from the vertex.

9. Round the circumference of a circle $n$ equal centres of force are ranged symmetrically; each force is repulsive and varies inversely as the $m^{\text {th }}$ power of the distance. $\Lambda$ particle is placed in the plane of the circle very near its centre; shew that approximately the resultant force on it tends to the centre of the circle and varies as the distance of the particle from the centre, except when $m=1$.

10. Eight centres of force, resident in the corners of a cube, attract, according to the same law and with the same absolute intensity, a particle placed very near the centre of the cube; shew that their resultant attraction passes through the centre of the cube, unless the law of force be that of the inverse square of the distance.

11. If the law of force in the preceding example be that of the inverse square of the distance find the approximate value of the attraction on a particle placed very near the centre.

Result. Take the centre of the cube as origin and the axes parallel to the edges of the cube; then if $x, y, z$ be the coordinates of the particle the attraction parallel to the axis of $x$ is approximately

$$
\frac{56 x}{9(a \sqrt{3})^{5}}\left(3 y^{2}+3 z^{2}-2 x^{2}\right)
$$

towards the origin; $2 a$ being the length of an edge.

12. The attraction of a uniform rod of indefinite length on an external particle varies as (distance) $)^{-1}$ of the point from the 
rod. Prove this, and supposing the asymptotes of sn hyperbula to consist of such material, ahew that a particle will the in cyuilibrium at any point of the hyperbola, and that the jreanure on the curve at any point is proportional to the length of the tangent intercepted by the asymptotes.

13. An elliptic lamina attracts an internal particle $(x, y)$ with a force varying inversely as the distance; shew that if $X, Y$ be the whole attractions parallel to the axes,

$$
\frac{X}{x}+\frac{Y}{y}=\text { constant. }
$$

14. If $A, B, C$ be the attractions of an ellipanid in directions parallel to its axes on an internal particle situateal at the point $(f, g, h)$, shew that

$$
\frac{A}{j}+\frac{B}{g}+\frac{C}{h}=4 \pi \rho
$$

\section{(S.eo Arts. 228 and a39.)}

15. The resultant attraction of $\mathrm{n}$ particle which attracts according to the inverse cube of the distance on a filane lamina is the same as on that part of the spherical aluell described about the particle as centre and touching the plane of the lamina, which is cut off by straight limes from the contro to the edge of the lamina.

16. A particle attracted by two centres of force at $A$ and $B$ is placed in a fixed groove. Shew that the particle remains at rest at whatever point it is placed, provided that the form of the groove be such that

$$
(A P-c)(B P-c)=c^{\circ} \text {, }
$$

where $c, c^{\prime}$ are constants dependent upon the absolute forces.

17. If a portion of a thin spherical shell, whose projections upon the three co-ordinate planes through the centre are $A, B, C$, atract a particle at the centre with a force rarying as any function of the distance, shew that the particle will le gin to move in the direction of the straight line whose cquatiuns are

$$
\frac{x}{A}=\frac{y}{B}=\frac{z}{C}
$$


18. The particles of a thin hemispherical shell attract with a force $=\mu$ (distance), and those of a right conical shell repel with a force $=\mu$ (distance). 'The rims of their bases coincide, and their vertices are turned in opposite directions, shew that a particle will rest in the common axis produced at a distance from the vertex of the sphere = length of the axis of the cone, the vertical angle of the cone being $2 \tan ^{-1} \frac{4}{3}$.

19. Shew that if the attraction vary inversely as the distance an indefinitely thin plane ring exerts no force on a particle in the plane of the ring within its inner circumference.

[This and the following example depend on the integral

$$
\int_{0}^{\pi} \frac{(a-c \cos \theta) d \theta}{a^{3}+c^{2}-2 a c \cos \theta}
$$

for which see Integral Calculus, Chapter Iv.]

20. Shew that if the attraction vary inversely as the distance an indefinitely thin plane ring attracts a particle in the plane of the ring beyond its outer circumference in the same manner as if the mass of the ring were collected at its centre.

21. If a straight line be the attracting body, shew that the lines of force are hyperbolas and the surfaces of equilibrium spheroids. (Cambridge and Dublin Mathematical Journal, Vol. III. p. 94.)

22. From the proposition established in Art. 244, deduce that established in Art. 239. (Cambridge and Dublin Mathematical Journal, Vol. v. p. 215.) 


\section{CIIAPTER XIV.}

\section{VIKTUAL VELOCIT\&FS.}

247. Wr: proceed to establish a general thoorem respect. ing the equilibrium of a body or system of bodies, called the Principle of Virtual Velocities.

When a system of particles is in equilibrium, and we suppose each of them placed in a position indefinitely near that which it really oecupies, without disturbing the connexion of the parts of the system with each other, the straight line which joins the first position of a particle with the scound is called the virtual velucity of that particle.

The term velocity is used because we may conecive all the displacements to the made in the same indefinitely small time, and then the spaces described are proportional to the velocities The word virfual is used to intmate that the displacements are not really made, but only supposed. We retain the established phraseology, but it is evident from these explanations that the words virtual velocity might be conveniently replaced by hypothetical displacement.

By the words, without disturbing the conncxion of the parts of the system with each other, we mean, that any rigid body which exists in the system is supposed to remain of invariable form, and that any rods or strings which connect different parts of the system are to remain unbroken. This, at least, will serve for a preliminary statement to assist the reader, and we shall recur to the subject again; see Art. 257. Ilence, by reason of this limitation the virtual velocities of the different parts of a system are frequently so connected that when those of a definite number of points are assumed, thuse of all the rest necessarily follow.

243. The virtual velocity of a particle estimated in a given direction is the prejection of the virtual velweity on this direction; it is cunsidered pusitive when the direction 
of the motion of the particle, in passing from its first position to its sccond, makes an acute angle with that along which we are estimating the velocity. Thus the virtual velocity of a particle estimated along any given straight line is found both in magnitude and sign, by multiplying the absolute virtual velocity by the cosine of the angle which its direction makes with the given straight line.

The virtual moment of a force is the product of its intensity by the virtual velocity of its point of application estimated in the direction of the force.

We can now enunciate the principle of virtual velocities.

If any system of particles is in equilibrium, and we conceive a displacement of all the particles which is consistent with the conditions to which they are subject, the sum of the virtual moments of all the forces is zero, whatever be the displacement. And conversely, if this relation hold for all the virtual displacements, the system is in equilibrium.

249. The student will derive from the demonstrations which follow a better notion of the meaning of the principle than from the mere enunciation of it; it is, in fact, necessary to obtain a general view of the whole subject before attempting fully to comprehend the preliminary definitions and statements. One remark may be made for the purpose of anticipating a difficulty; each virtual moment is by definition an indefinitely small quantity, that is, ultimately vanishes, so that the principle seems to amount only to this, take each force of the system and multiply it by a quantity which ultimately vanishes, then the sum of these products vanishes. 'The principle, however, implies more than this statement, as we shall see.

The convenient term virtual moment is given by Duhamel; it may, however, be useful to enunciate the principle of virtual velocities without introducing this term, and we therefore give the following.

Suppose a material system held in equilibrium by any forces, and suppose the points of application of the forces moved through very small spaces in a manner consistent with the connexion of the parts of the system with each 
other. Let perpendienlan be drawn from the new positions of the points on the directions of the forces acting at the points in their pasitions of equilibrium. The distance between the foot of any prerpendicular and the original point of application of the correnponling force, is called the virtaal velocity of the point with respert to that fores, and is estimated positive or negative, aceoriling as the perpendicular falls on the side of the point towards which the force acts or on the opposite side. Then the principle is this, the aligebraical sum of the product of ench force of the system and the corresponding virtual velocity wanishes. And conversely, if the sum vanishes for every displacement the system is in equilitrium.

Befire we proceed to a general demonstration, we will consider two simple cases, that of a particle, and that of a rigil roul acted on by forces at its ends.

250. Suppose that forces act on a single particle and maintain it in equilibrium. Let $P_{3}, P_{v} \ldots$ denote the fores: $a_{1}, a, \ldots$ the angles which their directions respectively make with any fixed straight line arbitrarily chosen; then, by Art.29,

$$
\Sigma P \cos \alpha=0 \text {. }
$$

If every term of this equation be multiplied by the arhitrary quantity $r$, we have $\Sigma \operatorname{Pr} \cos \alpha=0$. But $r \cos \alpha_{1}$ is the projection of the length $r$, measured along the fixed line, on the direction of the force $P_{1}$; a similar meaning may be assigned to $r \cos a_{2}, r \cos a_{3}, \ldots$ Also $r$ may be considered as the distance of the first position of the particle from a second position arbitrarily elusen, and therefore, when $r$ is indefinitely diminished, $r \cos \alpha_{1}, r \cos a_{2}, \ldots$ become the rirtual relocities of the particle with respect to $P_{1}, P_{0}, \ldots$ Hence, the principle holds in this couse.

Conversely, if $\mathbf{I} F \cos \alpha=0$ for all directions of displacement; then, $Y P \cos \alpha=0$ for all direetions, and the particle is in equilibrium under the action of the given forces.

In this case, we observe that the hypothetical displacement of the particle may be of any magnitnde we please, and that the sum of the products of each force into the projection of the displacement on its direction is not only ultimately, but alwoays sero. 
251. Since when a system of forces acting on a particle is in equilibrium, each force is equal and opposite to the resultant of all the other forces, and, as we have just seen, the sum of the products of each force into its virtual velocity is zero, it follows, that the product of any force into its virtual velocity is numerically equal to the sum of such products for any system of forces which it balances, but is of the opposite sign. Hence if a single force is the resultant of a system of forces acting at a point the product of the single force into its virtual velocity is equal to the sum of such products for the system of forces.

252. Next, suppose a rigid rod acted on by a force at each end. Let $x, y, z$ be the co-ordinates of one end, and $x^{\prime}, y^{\prime}, z^{\prime}$ those of the other; $l$ the length of the rod; then

$$
\left(x-x^{\prime}\right)^{2}+\left(y-y^{\prime}\right)^{2}+\left(z-z^{\prime}\right)^{2}=l^{2} \ldots \ldots \ldots \ldots(1) .
$$

Suppose the rod displaced; let $\delta x, \delta y, \delta z$ be the changes made in the co-ordinates of one end; $\delta x^{\prime}, \delta y^{\prime}, \delta z^{\prime}$ those made in the co-ordinates of the other end; then

$$
\begin{aligned}
& \left(x+\delta x-x^{\prime}-\delta x^{\prime}\right)^{2}+\left(y+\delta y-y^{\prime}-\delta y^{\prime}\right)^{2}+\left(z+\delta z-z^{\prime}-\delta z^{\prime}\right)^{2}=l^{2} \ldots(2) . \\
& \text { From (1) and }(2), \\
& \begin{array}{l}
2\left(x-x^{\prime}\right)\left(\delta x-\delta x^{\prime}\right)+2\left(y-y^{\prime}\right)\left(\delta y-\delta y^{\prime}\right)+2\left(z-z^{\prime}\right)\left(\delta z-\delta z^{\prime}\right) \\
+\left(\delta x-\delta x^{\prime}\right)^{2}+\left(\delta y-\delta y^{\prime}\right)^{2}+\left(\delta z-\delta z^{\prime}\right)^{2}=0 \ldots \ldots \ldots(3) .
\end{array}
\end{aligned}
$$

Let $\alpha, \beta, \gamma$ be the angles which the original direction of the rod makes with the axes; then

$$
x^{\prime}-x=l \cos \alpha, y^{\prime}-y=l \cos \beta, z^{\prime}-z=l \cos \gamma \ldots \text { (4). }
$$

If then, in (3), we neglect the terms $\left(\delta x-\delta x^{\prime}\right)^{2},\left(\delta y-\delta y^{\prime}\right)^{2}$, $\left(\delta z-\delta z^{\prime}\right)^{2}$ in comparison with those we retain, we have

$$
\left(x-x^{\prime}\right)\left(\delta x-\delta x^{\prime}\right)+\left(y-y^{\prime}\right)\left(\delta y-\delta y^{\prime}\right)+\left(z-z^{\prime}\right)\left(\delta z-\delta z^{\prime}\right)=0,
$$

or, by means of (4),

$$
\delta x \cos \alpha+\delta y \cos \beta+\delta z \cos \gamma=\delta x^{\prime} \cos \alpha+\delta y^{\prime} \cos \beta+\delta z^{\prime} \cos \gamma \ldots \text { (5). }
$$

Suppose $P$ the resultant of the forces acting at one end of the rod, and $P^{\prime}$ the resultant of those acting at the other end; then, in order that there may be equilibrium, these forces 
must be cyual in magnitude and must act along the rod in opposite disections. This is strions, or may be easily shewn by Art. 73. Since then $F^{\prime}=-I$, we have by (5)

$P(\delta x \cos a+\delta y \cos \beta+\delta=\cos \gamma)$

$$
+P\left(\delta x^{\prime} \cos \alpha+\delta y^{\prime} \cos \beta+\delta z^{\prime} \cos \gamma\right)=0 \ldots \ldots(6) \text {. }
$$

Since $P$ acts along the rod, the first term is the product of $P$ into the resolved virtual velocity of its point of application, and the socond term is a similar prodnet for $I^{2}$; hence, the principle of virtnal velocities holds in this case.

The converse of this theorcm is true in this case, but we shall not give a separate demonstration of it: the general demonstration of Art. 253 will sufticiently illustrate this point.

If (3) were absolutely true, then in the case of a rol, as in that of a single particle, the sum of the products of each force into the projection of the displacement of its point of applicastion on the direction of the force wonld be zero, whether the displacement were finite or infinitesimal. But (5) insteal of being absolutely true is obtained from (3) by neglecting squares and products of the resolved displacements $\delta x, \delta s, \delta y, .$.

253. We proceed to establish the truth of the principle in the case of a rigid body. We shall assume that any possible displacement of a rigid body may be produced, by first making the body rotate about some axis, and then moring all the particles of the body through equal spaces in parallel directions. Sce Spherioal Trigomometry, Chapter XuII. Suppose, for simplicity, that the axis of $z$ is male to coincide with the axis about which the body is turned; let $\theta$ be the angle through which the body is turned, then the co-ordinates of a particle which were originally $x$ and $y$ will become, if ore neglect the square and higher poucers of $\theta, x-y \theta$ and $y+x \theta$ respectively; the co-ordinate $z$ of the particle remains unchanged.

Tet the body bo now further displaced, so that each particle moves threngli a space of which $a, b, c$ are the projections on 
the co-ordinate axes; then, if $\delta x, \delta y, \delta z$ denote the vhole changes made in the co-ordinates $x, y, z$ of a particle, we have

$$
\delta x=a-y \theta, \quad \delta y=b+x \theta, \quad \delta z=c .
$$

Since the forers which act on the rigid body are supposed to keep it in equilibrium, we have by $\hat{\Lambda}$ rt. 73 ,

$$
\begin{gathered}
\Sigma X=0, \quad \Sigma Y=0, \quad \Sigma Z=0, \\
\Sigma(Z y-Y z)=0, \quad \Sigma(X z-Z x)=0, \quad \Sigma\left(Y x-X_{y}\right)=0 .
\end{gathered}
$$

Multiply the first of these equations by $a$, the second by $b$, the third by $c$, and the sixth by $\theta$, and add; we then find

$$
\Sigma\{X(a-y \theta)+Y(b+x \theta)+Z c\}=0,
$$

or $\quad \Sigma(X \delta x+Y \delta y+Z \delta z)=0$.

Let $P_{1}$ denote the force of which $X_{1}, Y_{1}, Z_{1}$ are the components, and $P_{2}, P_{3}, \ldots \ldots$ have similar meanings; and let $\delta p_{1}, \delta p_{2}, \ldots \ldots$ be the resolved virtual velocities corresponding to these forces; then, by Art. 250, the above equation may be written

$$
\Sigma P o p=0 \text {. }
$$

This proves the principle in the case of a rigid body.

Conversely, if the sum of the products of the forces and the resolved virtual velocities vanishes for every possible displacement of a rigid body, the forees keep the body in equilibrium.

For suppose, in the first place, the body is so displaced that every point of it moves parallel to the axis of $x$ over a space $a$; then we have, by hypothesis,

$$
\Sigma X a=0 \text {; }
$$

therefore

$$
\Sigma X=0 \text {. }
$$

Similarly, by suitable displacements, we may prove that

$$
\Sigma Y=0 \text {, and } \Sigma Z=0 \text {. }
$$

Next, suppose the body turned round the axis of $z$ through a small angle $\theta$; then, by hypothesis,

$$
\Sigma(X \delta x+Y \delta y)=0
$$


and

$$
\delta_{x}=-y \theta, \quad \delta y=x \theta \text {; }
$$

therefore

$$
\theta \Sigma(X y-Y x)=0 \text {; }
$$

therrfore

$$
\Sigma(\gamma x-X y)=0 \text {. }
$$

Similarly, by suitable displacements, we may jorove

$$
\Sigma(Z y-Y z)=0, \quad \Sigma(X z-Z x)=0 \text {. }
$$

Hence, the six equations of equilibrium bold.

If there be a system of two or more rigid bodies, then, since the principle of virmal velocities holds for any possible displacrment of any one of the boties, it holds for any possible displacement of the system.

25.4. In Art. 252 we have simplificd the proof of the first part of the principle of virtual velocities, by supposing the axis of $z$ to coincide with that about which tho boly was male to und.rgo an angular displacement. The following will be the prossss, if we suppose the displacencnt male about a straight line passing through the origin, and inclined to the axis at angles whose dirccion cosines are $l, m, n$.

I. $r$ be the distance of any point $(x, y, z)$ from the origin; $\phi$ the angle this distance makes with the given straight life; $p$ the propendicular from $(x, y, z)$ on the given straight lise; then

$$
\begin{gathered}
r^{2}=x^{2}+y^{2}+z^{2}, \\
\cos \phi=\frac{7 x}{r}+\frac{m y}{r}+\frac{n z}{r} ;
\end{gathered}
$$

therefore $\rho^{2}$ or $r^{2} \sin ^{2} \phi=x^{2}+y^{2}+z^{2}-(l x+m y+n z)^{2}$.

Suppose the body turned through a small angle $\theta$ round the given line; let $x+\delta r, y+\delta y, z+\delta z$, be the co-ordinates of that point of the body which was uriginally at $(x, y, z)$.

Since $r$ and $\rho$ are unchanged by the displacement, we have, by neglecting $(\delta x)^{2},(\delta y)^{2},\left(\delta y^{3}\right.$ in comparison with $\delta x, \delta y, \delta$,

$$
\begin{aligned}
& 0=x \delta x+y \delta y+x \delta \\
& 0=16 x+m \delta y+m \delta s
\end{aligned}
$$

therefore $\frac{\delta x}{y n-z m}=\frac{\delta y}{z l-x n}=\frac{\delta s}{x m-y l}=\lambda$ suppose......(1). 
And since $\quad\left\{(\delta x)^{2}+(\delta y)^{2}+(\delta z)^{2}\right\}^{\delta}=2 \rho \sin \frac{1}{2} \theta$,

or

$$
\lambda\left\{(y n-z m)^{2}+(z l-x n)^{2}+(x m-y l)^{2}\right\}^{2}=2 \rho \sin \frac{1}{2} \theta,
$$

therefore

$$
\lambda\left\{x^{2}+y^{2}+z^{2}-(l x+m y+n z)^{2}\right\}^{3}=2 \rho \sin \frac{1}{2} \theta ;
$$

neglecting $\theta^{3}$ and higher powers of $\theta$.

Suppose the body to be further displaced, so that each particle moves over spaces $a, b, c$ parallel to the co-ordinate axes; if $\delta x, \delta y, \delta z$ denote now the whole displacement of the particle whose original co-ordinates were $x, y, z$, we have

$$
\begin{aligned}
& \delta x=(y n-z m) \theta+a, \\
& \delta y=(z l-x n) \theta+b, \\
& \delta z=(x m-y l) \theta+c .
\end{aligned}
$$

Multiply the six equations in $\Lambda$ rt. 73 by $a, b, c,-l \theta,-m \theta$, $-n \theta$, respectively, and add, then

$$
\Sigma(X \delta x+Y \delta y+Z \delta z)=0 .
$$

255. We shall illustrate the principle of virtual velocities in the solution of the following problem.

$\Lambda$ beam in a vertical plane rests on a post $B$ and against a wall at $A$; required the circumstances of equilibrium.

Let the distance of $B$ from the wall $=b$; let $G$ be the centre of gravity of the beam; $A G=a$; and the inclination of the beam to the wall $=\theta$. The reaction $\left(P^{\prime}\right)$ of the post at $B$ is

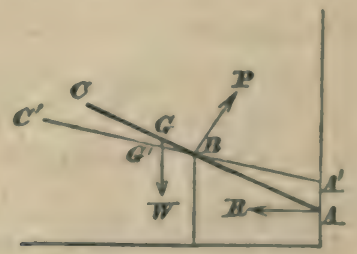

perpendicular to the surfaces in contact, and therefore to the beam; the reaction $(R)$ of the wall is perpendicular to the wall for the same reason; let $W$ be the weight of the beam. We may consider the beam in equilibrium under the action of $P, R, W$, and suppose the post and wall removed. 
Now the object of the problem might be, solely to deter: mine the pasition of equilibrium, or also to determine $P$ and not $R$, or $R$ and not $P$, or to determine both $P$ and $R$ and also the pasition of equilibrium. We shall solve the problem by the principle of virtual velocities under these four suppositions, in order to explain the method of proceeding so as to avoid as mnch tronble as possible according to the nature of the question.

(1) Suppose the position of equilibrimn only required. We must then give the bean a small arbitrary geometric motion such that the unknown pressures $P$ and $R$ shall not occur in the equation of virtual velocities; the beam must therefore remain in contact with the wall and the post, as in the figure.

Let $\delta \theta$ be the incrense of $\theta$ owing to the displacement. Then the height of $G$ above the horizontal straight line through $B$, (or $z$ ), before displacement

$$
=G B \cos \theta=(a-b \operatorname{cosec} \theta) \cos \theta=a \cos \theta-b \cot \theta \text {; }
$$

the height after displacement is found by changing $\theta$ into $\theta+\delta \theta$ in this expression; therefore, the rertical space described by $\bar{G}$ or $\delta z$

$$
\begin{gathered}
=a \cos (\theta+\delta \theta)-b \cot (\theta+\delta \theta)-(a \cos \theta-b \cot \theta) \\
=\left(\frac{b}{\sin ^{2} \theta}-a \sin \theta\right) \delta \theta ;
\end{gathered}
$$

and, by the principle of virtual velocities, $W \delta=0$; therefore

$$
b-a \sin ^{2} \theta=0, \quad \sin \theta=\sqrt[3]{\frac{b}{a}},
$$

and this determines the position of equilitrium.

(2) But suppose we wish to find the pressure $P$ as well as the position of equilibrium.

We must in this care move the beam off the post, in order that the virtual velocity of $B$ with respect to $P$ may not vanish, and consequently $P$ not disappear as in the first case.

Let $A A^{\circ}=c$, and let, as before, $\delta \theta$ be the change of $\theta$. 
We have to find the displacement of $B$ estimated along

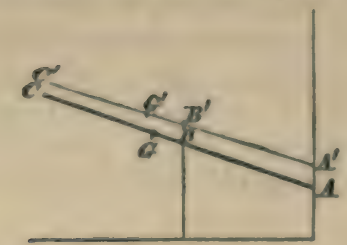

the line of action of $P$. Now conceive the beam brought into its second position by two stejs; first let it be moved parallel to itself till the lower end comes to $A^{\prime}$, and next let it revolve round $A^{\prime}$ through a small angle $\delta \theta$. By the first step $B$ moves through a space parallel and equal to $A A^{\prime}$; by the second step $B$ describes a small are of a circle the length of which is $A B \cdot \delta \theta$, that is $b \operatorname{cosec} \theta \delta \theta$. Thus the displacement of $B$ estimated along the line of action of $P$ is ultimately $c \sin \theta-b \operatorname{cosec} \theta \delta \theta$.

Similarly by the first step $G$ moves through a space equal and parallel to $A A^{\prime}$, and by the second step $G$ describes a small arc of a circle the length of which is $a \delta \theta$. Thus the displacement of $G$ resolved vertically downwards is ultimately $a \delta \theta \sin \theta-c$.

Therefore, by the principle of virtual velocitics, $W(a \sin \theta \delta \theta-c)+P(c \sin \theta-b \operatorname{cosec} \theta \delta \theta)=0 ;$

therefore, $\delta \theta(W a \sin \theta-P b \operatorname{cosec} \theta)-c(W-P \sin \theta)=0$; and, since $c$ and $\delta \theta$ may be any independent small quantities, $W a \sin \theta-P b \operatorname{cosec} \theta=0, \quad W-P \sin \theta=0 ;$

therefore

$$
\sin \theta=\sqrt[3]{\frac{b}{a}} \text {, and } \frac{P}{W}=\sqrt{\frac{a}{b}} \text {. }
$$

(3) Suppose we wish to know $R$ and the position of equilibrium, and not $P$.

Then we should displace the beam 80 as to give to $A$ a virtual velocity with respect to $R$, but not one to $B$ with respect to $P$.

The beam must therefore still remain in contact with the peg. Let $A A^{\prime}=c$, and let $\alpha$ be the angle which $A A^{\prime}$ makes 
with the vertical. Now conceive the beam brought into ita second position by two steps; first let it be moved parallel

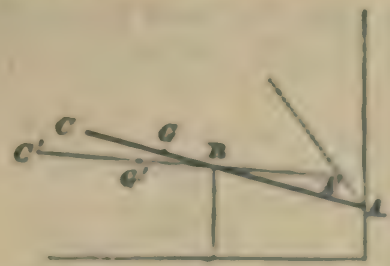

to itself till the lower end comes to $A^{\prime}$, nnd next let it revolve round $A$ through an angle $\delta \theta$ so as to bring the beam again into contact with the peg. The displacennent of $A$ estimated alung the line of action of $R$ is osin $\alpha$. The displacement of $Q$ estimated vertically dowuwards is $a \delta \theta \sin \theta-c \cos \alpha$.

Moreover there is a relation between $\varepsilon \theta, c$, and $\alpha_{\text {, arising }}$ from the fact that the whole displacement of the beam is such as to keep the beam still in contact with the [eg. From the triangle $A R A$, we have

$$
\frac{\sin 8 \theta}{\sin (\theta-\alpha)}=\frac{A A^{\prime}}{A^{\prime} B^{\prime}}
$$

hence,

$$
\delta \theta=\frac{c \sin (\theta-a) \sin \theta}{b} \text { ultimately. }
$$

Therefore by the principle of virtual relocities

that is,

$$
W\left\{\frac{a c}{6} \sin ^{2} \theta \sin (\theta-a)-c \cos \alpha\right\}+R \operatorname{cosin} \alpha=0 ;
$$

$$
\Pi\left(\frac{a \sin ^{2} \theta}{b}-1\right) c \cos \alpha+\left(R-\frac{W a}{b} \sin ^{2} \theta \cos \theta\right) c \sin \alpha=0 ;
$$

and $c \cos \alpha$ and $c \sin \alpha$ are independent; thereforo

$$
\frac{a \sin ^{2} \theta}{b}-1=0, \quad R-\frac{W_{a}}{b} \sin ^{2} \theta \cos \theta=0 ;
$$

therefore $\sin \theta=\sqrt{ }^{\prime} \frac{b}{a}$, and $\frac{I f}{W^{3}}=\frac{\sqrt{\left(a^{3}-b^{3}\right)}}{b^{3}}$. 
(4) Lastly, suppose we wish to determine $P$ and $R$ and the position of equilibrium.

Then we must give the beam the most general displacement possible in the plane of the forces; let $A A^{\prime}=c$, and

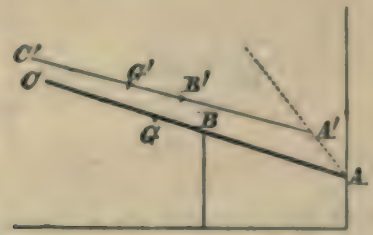

let $\alpha$ be the angle which $A A^{\prime}$ makes with the vertical. Now conceive the beam brought into its second position by two steps; first let it be moved parallel to itself till the lower end comes to $A^{\prime}$, and next let it revolve round $A^{\prime}$ through an angle $\delta \theta$. The displacement of $A$ estimated along the line of action of $R$ is $c \sin \alpha$. The displacement of $G$ estimated vertically downwards is

$$
a \delta \theta \sin \theta-c \cos \alpha .
$$

The displacement of $B$ along the line of action of $P$ is

$$
c \cos \left(\alpha+\frac{\pi}{2}-\theta\right)-b \operatorname{cosec} \theta \delta \theta
$$

that is,

$$
c \sin (\theta-a)-b \operatorname{cosec} \theta \delta \theta \text {. }
$$

Therefore by the principle of virtual velocities

$$
\begin{aligned}
& W(a \delta \theta \sin \theta-c \cos \alpha)+R c \sin \alpha \\
& +P\{c \sin (\theta-\alpha)-b \operatorname{cosec} \theta \delta \theta\}=0 ;
\end{aligned}
$$

that is,

$(W a \sin \theta-P b \operatorname{cosec} \theta) \delta \theta+(P \sin \theta-W) c \cos \alpha$

$$
+(R-P \cos \theta) c \sin \alpha=0,
$$

and $\delta \theta, c \cos \alpha$, and $c \sin \alpha$ are independent; therefore $W a \sin \theta-P b \operatorname{cosec} \theta=0, P \sin \theta-W=0, R-P \cos \theta=0$. 
These three equations are the equations which wo should have obtained by the principles of Art. 57 ; they give by elimination

$$
\sin \theta=\sqrt{\frac{b}{a}}, \frac{P}{W}=\left(\frac{a}{b}\right)^{b}, \frac{R}{W}=\frac{\sqrt{\left(a^{3}-b^{3}\right)}}{w^{d}} .
$$

We have thus illustrated the method of application of this principle; and we observe, in general, that when the object of the problem does not require certain unknown forces, we must give the body the most arlitrary geometrical motion possible without giving the points of application of these torces any motion in their directions.

256. In applying the principle of virtual velocities to deduce the conditions of equilibrium of any system, it is often convenient to give the body such a displacement as to make the virtual moments of some of the furces soparately vanish. 'This has been excmplified in the preceding Article, and we will now enumerate some cases in which the virtual monent of a force vanishes.

(1) In the lypothetionl displacement, if any particles of the system have remained in their original places, the virtmal moment of forces acting at such points is obviously zero. If a body, for example, have one point fixed, then the rirtual velocity of this point is zero for any hypothetical displacement of the body, which does not lreak the condition of this point being fixed.

(2) Suppose a body compelled to remain with one point in contact with a smouth fixel plane, so that the plane exerts a force on the body at the point of contact in a direction perjendienlar to the plane. Let the benly be displaced so as to have the same point still in contact with the tixed plane, then the perpendicular dirawn from the new position of the point of contact on the old direction of the action of the fised plane meets that direction at the old pocition of the point of contact; that is, the virtual velocity of the point of contact relative to the force exerted by the plane is zero.

Similarly, if the body have more than one point in contact with the plane, and be so displaced that the same poinis of the body remain in contact with the fixed plane, the T. 8. 
virtual moment of each force which the plane excrts on the body vanishes.

(3) Let two smooth bodies be in contact; then each exerts a force on the other along their common normal. Suppose one of them so displaced, that the point in it which was originally in contact with the other body still remains in contact with it; the case is similar to that of a body in contact with a fixed plane; the virtual velocity of the point of contact relative to the normal force is not zero, but is indefinitely small compared with the absolute virtual velocity.

Let $B A C$ be a section of one body made by a plane which

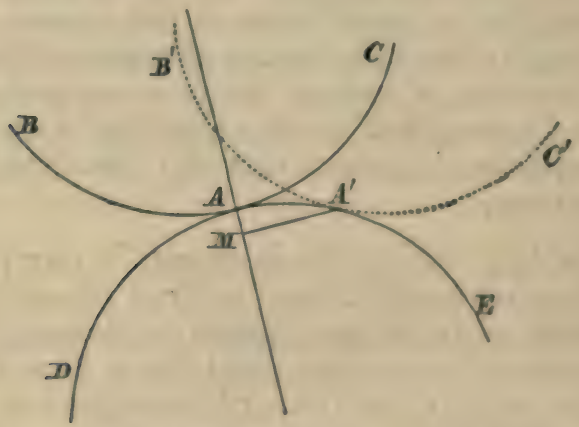

contains the common normal to the surfaces, and $D A E$ the section of the other made by the same plane; $A$ the point of contact. Suppose the body $B A C$ displaced into the position $B^{\prime} A^{\prime} C^{\prime}$, so that the point $A$ is moved to $A^{\prime}$. Draw $A^{\prime} . M$ perpendicular to the common normal to the surfaces. Then $A M$ represents the virtual velocity of the point of contact with respect to the normal force, while the straight line joining $A$ and $A^{\prime}$ is the absolute virtual velocity. Since $M A A^{\prime}$ is ultimatcly a right angle, $A M$ vanishes compared with $A \Lambda^{\prime}$.

(4) Suppose two bodies in contact at a single point, and let them be both displaced so that they still remain with the same point of each body in contact. Let $P$ denote the force in the normal on one body, and therefore $-P$ that on the other; then, if $P \delta p$ denote the virtual moment of the normal 
force with respect to the first body, - Pôp will be the virtual moment with respect to the secotsd body. Hence, by taking the sum of the virtual monents for the two bodies, the mutual action $P$ disappears.

A similar result holds if the bodies be in contact at more points thas one.

(5). Suppose a body in contrat with a smooth fixed pilsue at a single point, and let the body be displaced by rolling it on the fixed plane.

Let $B A C$ be a section of the body made by a plane through

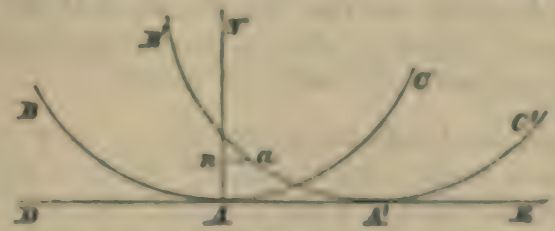

the point of contact $A$ containing the common normal to the surfaces, and suppose this section a circle. Let $D . A B$ be the intersection of this plane with the fixed smooth plane. Suppose $B A C$ the position of the borly after displacement, $A$ ' being the new point of contact, amd let $a$ be the point in the body which was originally in contact with the fixed smooth plane. Draw an perpendicular to the normal $A . N$; then, $A n$ represents the resolved virtual velucity of the point of contact with respect to the normal force. Now $A n$ is equal to the product of the chord $A^{\prime} a$ and the sine of the angle between this chord and $A^{\prime} A$; and as this angle is ultimately indefinitely small, $A n$ is indefinitely small compared with the chond $A^{\prime} a$, and therefore also compared with the are $A^{\prime} a$ or $A A^{\prime}$. Hence if we neglect powers of $A A^{\prime}$ higher than the first, the virtual moment of the furce along the normal acting at the point of contact is zero.

$A$ similar result holds if $B . A C, D . A E$ be any curves insteal of a circle and straight line respectively.

If a displacement is made up of two, one like that in the second case, and one like that in the present case, the fixed plane being smooth, the virtual moment of the force exerted by the plane will vamish. 
(6) Let us suppose the bodics in contact to be rough, and a displacement to be made by rolling one upon the other as in the preceding case. The action of each body on the other will not be directed along the normal $A N$, but may be resolved into two, one along $A N$ and the other at right angles to $A N$. The virtual moment of the former force vanishes, as we have shewn in the preceding case; and since the direction of the straight line joining $A$ and $a$ ultimately coincides with $A N$ and is therefore perpendicular to the second force, the virtual moment of the second force vanishes in the same manner as in the third case.

The result depends on the hypothesis that the bodies roll on each other; if there is sliding the virtual moment of the force at right angles to $A N$ will not vanish.

(7) Suppose an inextensible string to have one end attached to a fixed point, and the other end to a particle either isolated or forming part of a rigid body; one of the forces of the system is then the tension of this string which acts along its length. Let the particle be so displaced as to keep the string stretched, then it may pass from its first to its second position by moving over an are of a circle, and in the same manner as in the third case, we sce that the rirtual velocity of the particle with respect to the tension which the string exerts, is indefinitely small compared with the absolute virtual velocity of the particle. Hence, the tension of the string disappears from the erjuation of virtual velocities.

(8) Suppose an inextensible string connecting two particles of the system, and let the particles be displaced along the direction of the string, the string being kept stretched. Then, if one particle be displaced through a space $\delta p$, and $P$ denote the tension of the string, and therefore the force exerted by the string on this particle, $P^{\prime} \delta p$ is the virtual moment of the force which the string exerts on this particle; also $-P \delta p$ will be the virtual moment of the force which the string exerts on the second particle. Hence, by taking the sum of the virtual moments for the two particles, the tension of the string disappears from the equation of virtual velocities.

(9) If we suppose a further displacement of the system in the preceding case, by keeping one particle fixed and making 
the othep describo an are of a circle, then, by the seventh eases, the turnion of the string disappears from the equation of virtaal velocities.

By a combination of the displacements considered in the serenth and eighth cases, we can produce any displacement that the two particles can undergo, so long as the exing is kept stretched. Hence, the tension of a string connecting two particles disappears from the equation of virtual velocitics.

Wo have supposed the string to pass in a strnight line from one particle to the other, but the same result would hold if the string were deflected by passing through one or more stmooth fixed rings, supposing it always kept stretehed. The demonstration would not hold for an exlensible string.

257. We can now understand more distinctly the meaning of the words, trithout disturbing the connexion of the parts of the system wieth each other, which are introluced into the enunciation of the theorem. The theorem is shewn in Art. 250 to be true for a particle; if then we consider a rigid body to be a collection of particles held together by molocular forces, the theorem will hold for every displacement of the particles of the rigid body, prorided we include the molecular forces and estimate their several virtual moments. But from the demonstration in Art. 253 it appears that we need not consider the molecular forces, provided we give to the different particles such displacements only as are consistent with the unbroken rigidity of the body. So with respect to such forces as are enunciated in the preceding Article, we may, if we take them into consideration, give to the system any displacements we plesse; but if we do not take them into consideration, we wust give such displacements only as we can prove will not introduce the virtual moments of these forces. Hence, the words which we are explaining amount to a direction to be careful to include exery foree of the system, except such as we know have their virtual moments zero for the particular displacement we are considering.

255. The following example will shew how the principle of virtual velocities may assist in the solution of problens. Six equal rods are fastened together by hinges at each end, 
and one of the rods being supported in a horizontal position the opposite one is fastened to it by an elastic string joining their middle points; determine the tension of this string.

Let $W$ denote the weight of each rod, $T$ the tension of the string. Suppose the system displaced slightly so that the lowest rod descends vertically through a space $x$. Then it will be easily seen that the centre of gravity of each of the two rods which are adjacent to the highest rod descends through a space $\frac{x}{4}$; and the centre of gravity of each of the two rods which are adjacent to the lowest rod descends through a space $\frac{3 x}{4}$; the point of application of the tension on the lowest rod descends through a space $x$. Therefore by the principle of virtual velocities

$$
2 W \frac{x}{4}+2 W \frac{3 x}{4}+W x-T x=0 ;
$$

therefore

$$
T=3 W \text {. }
$$

The mutual actions at the hinges disappear from the equation furnished by the principle of virtual velocities, and thus the required result is readily obtained.

259. The following is the process by which we may deduce the equations of equilibrium of any system from the principle of virtual velocities.

Let $P_{1}, P_{8}, P_{3}, \ldots$ denote the forces which act on a system; $P_{1} \delta p_{1}, P_{2} \delta p_{3}, \ldots$ their respective virtual moments for any displacement; then, by the principle,

$$
P_{1} \delta p_{1}+P_{2} \delta p_{2}+P_{8} \delta p_{8}+\ldots=0 \ldots \ldots \ldots \ldots(1) .
$$

This equation we proceed to develope.

Let $\alpha_{1}, \beta_{2}, \gamma_{1}$ be the angles which the direction of $P_{1}$ makes with the co-ordinate axes; $x_{1}, y_{1}, z_{1}$ the co-ordinates of the point of application of $P_{1}$; then

$$
\delta p_{1}=\cos \alpha_{1} \delta x_{1}+\cos \beta_{1} \delta y_{1}+\cos \gamma_{1} \delta z_{1} \ldots \ldots \ldots \text { (2); }
$$

this is rigorously true, and similar equations hold for $\delta p_{2}$, $\delta p_{8}, \ldots$. 
Now, in consequence of the connexion of the system, for example, the rigidity of some parts of it, or the junction of parts by rodis or strings, relations will bold betwoen the coordinatis $x_{1}, y_{1}, z_{1}, x_{1}, y_{2}, z_{2}, \ldots$ in virtue of which all of them may be expressed in terms of a certain number of them; or all of them may be expressed in terms of certain othes independent co-ordinntes and angles.

Suppose $\xi_{1}, \xi_{2}, \xi_{3}, \ldots \phi_{1}, \phi_{1}, \phi_{2}, \ldots$ to denote these independent co-ordinates and angles. Then, if we nogleat the squares and products, and higher poocers of $\delta x_{1}, \delta y_{1} \ldots \delta \xi_{1}$, $\delta \xi_{3}, \ldots \delta \phi_{2}, \delta \phi_{2}, \ldots$, we shall obtain equations of the form

$$
\begin{aligned}
& \delta r_{1}=A_{1} \delta \xi_{1}+A_{1} \delta \xi_{2}+\ldots+a_{1} \delta \phi_{1}+a_{2} \delta \phi_{2}+\ldots \\
& \delta r_{2}=B_{1} \delta \xi_{1}+B_{3} \delta \xi_{2}+\ldots+b_{1} \delta \phi_{1}+b_{2} \delta \phi_{2}+\ldots,
\end{aligned}
$$

where $A, A_{v}, \ldots B, B_{q} \ldots a_{v}, a_{p} \ldots b_{1}, b_{z}, \ldots$ are functians of the variables, but do not contain the increments $\delta \xi_{t}, \delta \xi_{v} \ldots$ $\delta \phi_{0}, \delta \phi_{1} \ldots$

Let the values of $\delta x_{1}, \delta y_{1} \ldots$ be substituted in the equations of which (2) is the type, and then let the values of $\delta p_{1}, \delta p_{2}, \ldots$ be substituted in (1); this equation will take the form

$$
\left.Q_{1} \delta \xi_{1}+Q_{2} \delta \xi_{2}+\ldots+\eta_{1} \delta \phi_{1}+\eta_{2} \delta \phi_{2}+\ldots=0 \ldots \ldots / 3\right) \text {. }
$$

The conditions for the equilibrium of the system are

$$
Q_{1}=0, \quad Q_{1}=0, \ldots \quad q_{1}=0, \quad q_{2}=0 \ldots \ldots \ldots(4) .
$$

For since $\delta \xi_{1}, \delta \xi_{2}, \ldots \delta \phi_{1}, \delta \phi_{2}, \ldots$ are by supprsition independent, we might have given the body such a displacement as to leave $\xi_{2}, \xi_{3}, \ldots \phi_{1}, \phi_{2}, \ldots$ unchanged; and then (3) would roduce to

$$
Q_{1} \delta \xi_{1}=0 \text {; therdire } Q_{1}=0 \text {. }
$$

Similarly, we may shew that the other equations of ( 1 ) hold.

260. We will give a simple example in illustration of the method of the preceding Article. A string of given length lins one end fixed at a point in the line of intersection of two vertical planes at right angles to each other, and at the other end 
carries a heavy particle which is repelled from these planes by forces of which one is constant and the other varies as the distance from the plane; find the positions of equilibrium.

Take the vertical plane from which the particle is repelled by a constant force as the plane of $(x, z)$, and the other verticul plane as the plane of $(y, z)$; take the point to which the end of the string is fixed as the origin, and let the axis of $z$ be vertically downwards. Iet $x, y, z$ denote the co-ordinates of the particle in a position of equilibrium, and $l$ the length of the string. Let $W$ be the weight of the particle, $F$ the constant repulsive force, $\mu x$ the force which varies as the distance of the particle from the plane of $(y, z)$. Conceive the particle displaced into an adjacent position, the co-ordinates of which are $x+\delta x, y+\delta y, z+\delta z$. Then by the principle of virtual velocities

$$
\mu x \delta x+F \delta y+W \delta z=0
$$

the tension of the string has no virtual moment by $\Lambda$ rt. 256.

Also

therefore

$$
\begin{array}{r}
x^{2}+y^{2}+z^{2}=l^{2} . \\
x \delta x+y \delta y+z \delta z=0
\end{array}
$$

By (3) we can express $\delta z$ in terms of $\delta x$ and $\delta y$; thus (1) becomes

$$
\left(\mu x-\frac{W x}{z}\right) \delta x+\left(F-\frac{W y}{z}\right) \delta y=0 .
$$

Therefore

$$
\mu x-\frac{W x}{z}=0, \text { and } F-\frac{W y}{z}=0 .
$$

From the first of these equations we obtain either $z=\frac{W}{\mu}$, or else $x=0$. If we take the former solution we obtain $y=\frac{F}{\mu}$, and then $x$ is known from (2) ; thus one position of equilibrium is determined. If we take the solution $x=0$, then $y$ and $z$ must be found from the equations

$$
F z-W y=0, \quad y^{2}+z^{2}=l^{2} ;
$$

thus another position of equilibrium is determined. 
261. The principle of virtual velucition is ueful in Btatics in the solution of wuch problems as that in Art. 235, where forees eceur which have their virtual moments zero for errais dirylacements. The following is an important general proposition to which the principle leads.

A system of riguil hedies under the action of no firces bus thirir weighes, mulual pressures, and pressurs npon smoolh imonines be sarfaces, veill be in equilitrium, if plasod so that the contre of grovity is in the lowest or highest position it oun jobs? ly athuin by moring the system consistently with the connexion of its parts with one another.

Let $s, s_{3}, \ldots$ denote the distances below a fixed horizonta! plane of the different particles of the syatem; $x_{1},{ }^{2}, \ldots, \ldots$ the weights of these particles. That the system may be in equilibrium, we must have

$$
r e_{2} \delta s_{1}+r_{s} \delta s_{3}+i r_{3} \delta s_{3}+\ldots=0 \ldots \ldots \ldots \ldots(1) \text {; }
$$

for by Art. 256 the virtual moments of all the other forces which act on the system vauish. Let z denote the degth of the centre of gravity of the system below the fixed horiz.ntal plane; then

$$
\bar{\Sigma}=\frac{w_{1} z_{1}+w_{1} z_{3}+w_{3} v_{3}+\ldots}{v_{1}+w_{3}+w_{3}+\ldots} ;
$$

therefore $\left(u_{1}+w_{2}+w_{1}+\ldots\right) \delta_{z}=w_{1} \delta z_{1}+w_{2} \delta_{s_{2}}+w_{0} \delta s_{1}+\ldots$ (2). Now when $z$ has a maximum or minimum value,

$$
\delta \bar{s}=0 \ldots \ldots \ldots \ldots \ldots \ldots \ldots \ldots(3),
$$

(sec Diff. Cale: Arts. 232, 238).

Hence, when the centre of gravity is at a maximum or minimum distance from the fixed horizontal plane, (I) is satisfied and the system is in equilibrium.

The ergution (3) is a necessury but not a sufficient condition for $z$ having a maximum or minimum value; hene, we cannot assert conversely, that when the system is in equilibrium, the ceutre of gravity must be at a maximum or minimum depth.

If the system of rigid bodies be such that the centre of gravity is always in the same horizontal plane, every position 
is a position of equilibrium. For in this case $\bar{z}$ is a constant, and therefore $\delta \bar{z}$ always $=0$.

If some of the bodies are rough the result will still hold if the friction be such as to prevent any sliding; see case (6) of Art. 256.

262. Suppose a system in equilibrium, and that an indefinitely small displacement is given to it; if it then tend to refurn to its original position, that position is said to be one of stable equilibrium; if the system tend to move further from its original position, that position is said to be one of unstable equilibrium.

To determine in any case whether the equilibrium of a system is stable or unstable, is a question of dynamics on which we do not enter. The reader may refer to Poisson, Art. 570 , or Duhamel, Tom. II. Art. 69 ; the best investigation of the question, however, will be found in the Cours Complémentaire d'Analyse et de Mécanique Rationelle, par J. Vieille, Paris, 1851.

The following general theorem is demonstrated. Suppose the forces which act upon a system such that

$$
\Sigma(X d x+Y d y+Z d z)
$$

is the immediate differential of some function of the co-ordinates, $\phi$; then, for every position of equilibrium, $\phi$ is, in general, a maximum or minimum; in the former case the equilibrium is stable and in the latter unstable.

An important particular case is that of the system in Art. 261 , in which the equilibrium is stable when the centre of gravity has its lowest position, and unstable when it has its highest position.

263. We will now illustrate the principle contained in the preceding Article by application to two examples.

I. A uniform heavy beam is placed with its ends in contact with a fixed smooth vertical curve in the form of an cllipse with its directrices horizontal: determine the position of stable equilibrium, the length of the beam being supposed not less than the latus rectum. 
Iet $P$ and $Q$ denote the extremities of the beam; Int $I^{\prime} M$ and $Q . N$ be perpendiculars on the lower directrix, and $S$ the focus corresponding to this directrix. Then the height of the centre of gravity of the beam above this directrix in $\frac{1}{2}(P . M+Q . V)$; for atable equilibrium this height shrould be a minimum. If o be the excentricity of the ellipse we have

$$
P M+Q N=\frac{1}{6}(S P+S Q) ;
$$

and therefore $S P+S Q$ must be a minimum. But $S P+S Q$ is always greater than $P Q$, except when $S$ is in the straight line $P Q$. Therefore the position of stable equilibrium is that in which $P Q$ passes through the focus.

Since the beam is in equilibrium under the action of its own weight and the normal resistances of the curve, it follows that the straight line which joins the point of intersection of normals at the ends of a focal chord of an ellipse with the middle point of the chord is parallel to the major axis : this result may be verified geometrically.

II. The principle of Art. 262 may be applied to a liquid which may be regarded as a collection of indefinitely small smooth heary particles.

Suppose a set of rectangles, all of the same length, but with any breadths. Let them be connected along their lengths by smooth hinges, so as to form a hollow prism without ends; and place the system vertically on a smowth horizontal plane. Let some liquid be poured into the vessel thus formed. In the position of stable equilibrium the centre of gravity of the liquid will be at a minimum height above the horizontal plane; and therefore the area of a horizontal section of the priam will then have a maximum value.

But by the principles of Hydrostatics the rectangles which form the vertical sides of the vessel are acted on by pressures from the fluid which form a system of forces like that in Prop. II. at the end of Chap. IV.: and therefore when there is equilibrium the horizontal section of the prism must form a polygon which can be inscribed in a circle. 
Hence we obtain the following result : if an area is to be bounded by given straight lines the area is greatest when the straight lines are all chords of a circle. See also Differentical Calculus, Art. 240.

264. The following is a simple example of distinguishing the nature of equilibrium.

$A$ heary lody rests on a fixed body, to determine the nature of the equilitrium; the surfaces bcing supposed rough.

Let $B A C$ be a vertical section of the upper body made

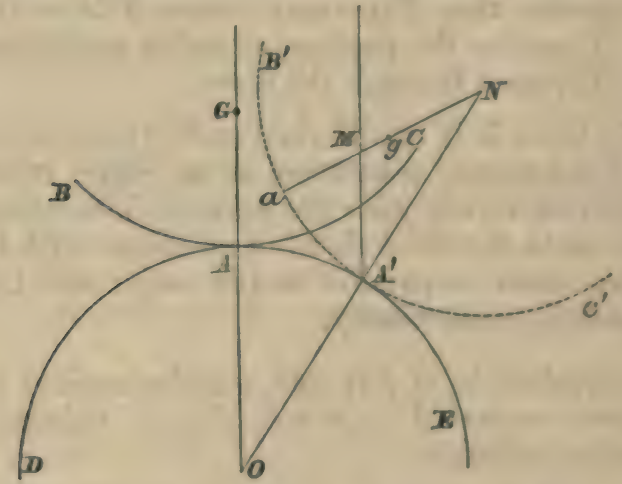

by a plane through its centre of gravity $G$, and $D A E$ the section of the lower body made by the same plane. We suppose these sections both circular; let $r$ be the radius of the upper section and $R$ that of the luwer. Let the upper body be displaced into the position $B^{\prime} A^{\prime} C^{\prime}$, and suppose $a$ that point in the upper body which was originally at $A$; at $A^{\prime}$ the new point of contact draw the common normal $O A^{\prime} N$, meeting at $O$ the radius $A O$ of the lower surface, and at $N$ the radius $a N$ of the upper surface. Draw a vertical line through $A^{\prime}$ meeting $a N$ at $M$; let $g$ be the new position of the centre of gravity of the upper body. If we suppose the surfaces rough enough to prevent all sliding, the upper body will turn round $A^{\prime}$, and the equilibrium will be unstable if $g$ falls further from $a$ than $M$, and stable if $g$ be between $M$ and $a$. 
Let $A O A^{\prime}=0, \quad a \cdot A^{\prime}=\phi$.

Since we strppose the upper body displaced by ralling on she lower, we have

$$
\operatorname{arc} A^{\prime}-\operatorname{arc} a A^{\prime} ;
$$

therefore

$$
r \theta=r \phi \text {. }
$$

Also

$$
\begin{aligned}
\frac{M N}{N A^{\prime}} & =\frac{\sin \theta}{\sin (\theta+\phi)}=\frac{\sin \theta}{\sin \left(1+\frac{K}{r}\right) \theta} \\
& =\frac{1}{1+\frac{l l}{r}} \text { ultimately; }
\end{aligned}
$$

therefore

$$
M N=\frac{r^{2}}{r+R^{2}},
$$

and

$$
a \cdot M=r-\frac{r^{\prime}}{r+18}=\frac{R r}{R+r} \text {. }
$$

Ilence, the equilibrium is stable or unstable according as $a g$, or $A G$, is less or greater than $\frac{l i r}{i+r}$.

If the lower surface be enncure instead of conrex, it may be shewn in the same way that the equilibrium is stable or unstable accorling as $A G$ is less or greater than $\frac{R r}{R-r}$.

The results of this $A$ rticle will hold when the sections BAC and $D A E$ are not circles; $r$ and $R$ will then stand for the radii of curvature of the upter and lower sections at the point $A$. If the lower surface is plane, $R$ is infinite, and $f:$ stable equilibrium $A C$ must be less than $r$.

$$
\text { 265. If } A G=\frac{R r}{R+r} \text { in the first crise, or }=\frac{R r}{R-r} \text { in the }
$$
second case, the equilibrium has been calleil noutrol. In this case, a further investigation will have to be made to determine whether the equilibrium is stalile or unstable. Suppoes, for example, that a portion of a paraboloid rests in neutral eqquilibrium with its vertex in contact with a horizontal plane, 
it is required to determine whether the equilibrium is stable or unstable.

Since the equilibrium is neutral, the centre of gravity $G$ must coincide with the centre of curvature of the generating parabola at the vertex; now, if different points be taken in a parabola, the further the assumed point is from the vertex, the further is the point of intersection of the normal and the axis from the vertex. Hence, the normal $A^{\prime} N$ in the figure meets

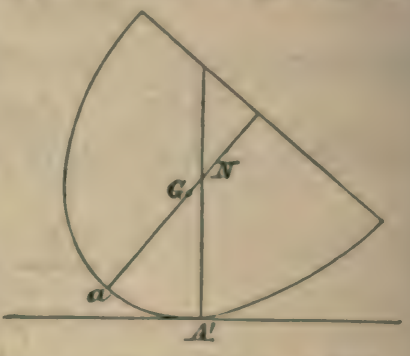
the axis of the parabola further from $a$ than $G$ is, and the equilibrium is stable.

It is easy to shew generally, that if a portion of a solid of revolution rest in neutral equilibrium with its vertex on a horizontal plane, the equilibrium is really stable or unstalile, according as the radius of curvature of the generating curve has a minimum or maximum value at the vertex.

266. The results of $A$ rt. 264, when the sections $B A C$ and $D A E$ are circles, may also be obtained by using the theorem which we have quoted in Art. 262.

Let $z$ denote the height of the centre of gravity $g$ above the horizontal line through $O$, and let $\mathrm{Ng}=c$; then

$$
\begin{aligned}
z & =(R+r) \cos \theta-c \cos (\theta+\phi) \\
& =(R+r) \cos \theta-c \cos \left(1+\frac{R}{r}\right) \theta .
\end{aligned}
$$

Expand the cosines in powers of the angles; thus

$$
\begin{aligned}
z=R+r-c & +\left\{c\left(1+\frac{R}{r}\right)^{2}-(R+r)\right\} \frac{\theta^{2}}{2} \\
& -\left\{c\left(1+\frac{R}{r}\right)^{4}-(R+r)\right\} \frac{\theta^{4}}{[4}+\ldots
\end{aligned}
$$


Suppose the coeflicient of $\theta^{\circ}$ not to be zero; then when $\theta$ is indefinitely small $z$ is greater of less than $R+r-c$ ar cording as the coefficient of 8 is positive or negative; in the former caso $R+r-c$ is a minimum value of $z$, and in the latter case it is a maximum value. Therefore the equitihrium is stuble if $c$ be greater than $\frac{r^{2}}{\Pi+r}$, and unstable if $c$ be leas than $\frac{r^{3}}{R+r}$.

Suppose however that $c=\frac{r^{2}}{R+r}$, then the coefficient of $\sigma^{\circ}$ is zero; in this case the equilibrium is said to be neutral. We must now examine the coefficient of $\theta^{\circ}$ in the value of $:$; this coefficient is

$$
\begin{array}{ll}
\text { that is, } & -\frac{1}{4}\left\{\frac{(R+r)^{3}}{r^{3}}-(R+r)\right\} ; \\
\text { that is, } & -\frac{R(R+r)(R+2 r)}{r^{3}[4}
\end{array}
$$

since this is a negatice quantity it follows that $n+r-c$ is a maximum value of $z$ and the equilibrium is really wnstable.

267. The following problem will furnish an instructive example. A frame formed of four uniform rods of the length a connected by smooth hinges, is hung over two smooth pigs in the same horizontal line at a listance $\frac{a}{\sqrt{2}}$, the two vega being in contact with different rods; shew that the frame is in equilibrium when each angle is $90^{\circ}$, and determine whether the equilibrium is stable or unstable.

Denote the pegs by $A$ and $B$; suppose the leam in contact with $A$ to make an angle $\theta$ with the horizan, and tlo bsam in contact with $B$ to make an angle $\phi$ with the horiass: let $u$ denote the depth of the centre of gravity of the system below $A B$. Then it may be sbewn that

$$
u=\frac{a}{2}(\sin \theta+\sin \phi)-\frac{\theta \sin \theta \sin \phi}{\sin (\theta+\phi)} \text {, }
$$


where

$$
c=\frac{a}{\sqrt{2}}
$$

Thus $u$ is a function of the two independent variables $\theta$ and $\phi$, and in order that $u$ may have a maximum or minimum value $\theta$ and $\phi$ must be taken so as to satisfy $\frac{d u}{d \theta}=0$ and $\frac{d u}{d \phi}=0$. It will be found on trial that $\theta=\frac{\pi}{4}$ and $\phi=\frac{\pi}{4}$ are suitable values. But it will be found that with these values for $\theta$ and $\phi$ we get

$$
\frac{d^{2} u}{d \theta^{2}}=-\frac{c}{2}, \quad \frac{d^{2} u}{d \theta d \phi}=-c, \quad \frac{d^{2} u}{d \phi^{4}}=-\frac{c}{2} ;
$$

so that $\left(\frac{d^{2} u}{d \theta d \phi}\right)^{2}-\frac{d^{2} u}{d \theta^{2}} \frac{d^{2} u}{d \phi^{2}}$ is positive and $u$ is neither a maximum nor a minimum when $\theta=\frac{\pi}{4}$ and $\phi=\frac{\pi}{4}$. All the foregoing is a simple example of the Differential Calculus; we proceed to apply it to the Mechanical Problem in question.

Let $\delta u$ denote the change in $u$ consequent upon changing the value of $\theta$ from $\frac{\pi}{4}$ to $\frac{\pi}{4}+\delta \theta$, and the value of $\phi$ from $\frac{\pi}{4}$ to $\frac{\pi}{4}+\delta \phi$; then it follows from the preceding investigations that

$$
\delta u=-\frac{c}{4}\left\{(\delta \theta)^{2}+4 \delta \theta \delta \phi+(\delta \phi)^{2}\right\}+\& c .
$$

where under the \&c. are included terms in $\delta \theta$ and $\delta \phi$ of a higher order than the second. Now although $u$ is neither a maximum nor a minimum when $\theta$ and $\phi$ are each $\frac{\pi}{4}$, yet there is equilibrium then because $\delta u$ is then zero so far as terms of the first order in $\delta \theta$ and $\delta \phi$. (See Art. 261.) But as $u$ is neither a maximum nor a minimum the equilibrium cannot be stated to be either stable or unstable universally; it is in fact stable with respect to some displacements and 
unstuble with respect to other displacomenta. If. fur esampite, we consider ouly such displacements as make $20=8$, then $\delta u$ is exrainly negative $w$ hen $\delta \theta$ and $\delta \phi$ are taken small enough ; thus the eentre of gravity is ruised by the displawe ment and so the cquilitrium is stable. If, again, we conbliter only such displacements as make $\delta \theta=-\delta \phi$, then $\delta$ is oestainly positive when $\delta \theta$ and $\delta \phi$ are taken small enongh; thus the centre of gravity is tepressed by the displacement and 80 the equilibrium is unstuble.

268. Of all curces of a given lengels draven bescren tuco fired points in a horizuntal line, the commen catenary in that which has its centre of grarity furthest from the atruight Tine joining the pointe.

This proposition belongs to the Calculus of Varviations, but an imperfect proof of it may be obtained from some of the preceding principles. Since the string which hangs in a common catenary is in equilibrium we conclude that the degth of its centre of gravity from the horizontal line is a maximum or minimum. (Sie however Art. 261.) And we may infer that the depth is a meximum and not a minimum from the experimental fact that if the string be slightly displacos it will return to its position of equilibrium so that its equilibrium is stuble. (see Art. 262.) Hence in any other prositiun of the string than that of equilibrium the centre of gravity will be nearer to the given horizontal line. And as the string which hangs in the common catenary is of uniform density and thickness its centre of gravity coincides with tlat of the curve. Thus the proposition is established.

269. Iagrange has given a demonstration of the principle of virtual velocitieg, which does not assume a knowledge of the conditions of equilibrium of any system of forcs: this demonstration is difficult and has not been unirersally received. We shall place it hete and refer the reader to Poisson, Art. 337, and to the article 'Virtual Velocities' in the Pemny Cyclopadia, for further information.

We have first to shew hew any system of furces may be replaced by a string in a state of tension passing numbl a combination of pullies.

T. s. 
Let forces $P, Q, R, \ldots \ldots$ acting at the points $A, B, C, \ldots \ldots$ maintain a system in equilibrium; let pullies be fixed to the system at the points $A, B, C, \ldots$ and let the pullies $a, b, c, \ldots$ be attached to fixed blucks, so that $A a$ may be the direction

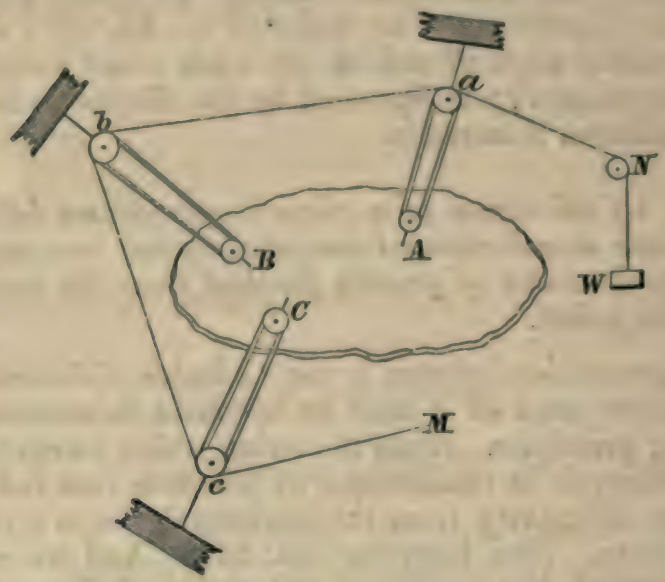

of the force $P, B b$ that of $Q$, and so on. Let a string have a weight $W$ attached to one end, and be passed round the pully $N$ and then round the pullies $a$ and $A$ a sufficient number of times to render the sum of the tensions equal to $P$. Let the same string then pass on to the pully $b$, and be passed round $b$ and $B$ a sufficient number of times, until the sum of the tensions is equal to $Q$. The string is then passed on to $c$, and round $c C$, and so on; the end of the string is fastened to a fixed point $M$. Thus the system of forces $P, Q, R, \ldots$ may be replaced by a single string, the tension of which is $W$. We here assume that the forces $P, Q, R, \ldots$ are commensurable.

We proceed now to the proof, in which we follow Lagrange's words very closely.

It is evident, in order that the system may remain in equilibrium, that the weight $W$ must be incapable of descending when any indefinitely small displacement whatever is given to the points of the system; for since the weight always tends to descend, if there were any displacement of the system which 
would allow it to dencend, it would necessarily descend and produce this displacement in the nyatem.

Let $a, \beta, \gamma, \ldots$ denote the indefinitely amall npraces, which any digylacement would cause the point of the system to deseritue in the direction of the forces, which respectively act at them, and let $p, q, r \ldots$ denote the number of parallel strings which are attuched to the pallies $A, B, C, \ldots$ Is is obrious that the spaces $\alpha, \beta, \gamma, \ldots$ are those by which the pullies $A, B, C, \ldots$ will approach $a, b, c_{1} \ldots$ and that the string joining these pullies will thus be diminished by $p_{2}, q_{3}, r y, \ldots$ Thus, in consequence of the inextensibility of the string, the weight $W$ would descend through the space $p a+q \beta+r \gamma+\ldots$ Hence, in order that the system of forces $P, Q, R, \ldots$ may be in equilibrium, we must have

$$
p a+q \beta+\eta \gamma+\ldots=0 ;
$$

and therefore, since $P=p W, Q=q W, \ldots$

$$
P_{z}+Q \beta+R_{\gamma}+\ldots=0 .
$$

This equation is the analytical expression of the principle of virtual velocities.

If the quantity $P_{x}+Q \beta+R_{\gamma}+\ldots$, instend of being zero, were negative, it might appear that this condition would be sufficient to ensure equilibrium, since it is impossible that the weight could of irself ascend? But we must remember, that whatever may be the connexion of the parts of the system, the relations which consequently hold between the indefinitely small quantities $\alpha, \beta, \gamma, \ldots$ can only be expressed by differential equations, and which are therefore linear as to these quantities: so that there will be necesarrily one or more of them which remain indeterminate and may be taken with a positive or negative sign; thus the ralaes of these quantities will be always such that they can simultaneously change their sign. Hence, it follows that if for a certain displacement of the system, the quantity $P a+Q \beta+l i \gamma+\ldots$ is negative, it would becone positive by changing the signas of $2, \beta, \gamma, \ldots$; thus the opposite displacement is equally jossible, and this would make the weight descend and destroy the equilibrium. 
Conversely, if the equation

$$
P \alpha+Q \beta+R \gamma+\ldots=0
$$

holds for every possible indefinitely small displacement of the system, it will remain in equilibrium. For, the weight remaining unmoved during these displacements, the forces which act on the system remain in the same condition, and there is no reason why they should produce one, rather than the other, of the two displacements, for which $\alpha, \beta, \gamma, \ldots$ have different signs. This is the case of a balance which remains in equilibrium, because there is no reason why it should incline to one side rather than the other.

The principle of virtual velocities being thas proved for commensurable forces, will also hold when the forces are incommensurable; for we know that any proposition which can be proved for commensurable quantities may be extended by a reductio ad absurdum to incommensurable quantities. 


\section{EXAMPIES.}

1. A cone whöso seni-vertical angle is $\tan ^{-1} \frac{1}{\sqrt{2}}$ in enclesed in the circumseribing spherical surface; shew that it will reat in any position.

2. A heavy uniform rod of length a moves in a rertical plane about a hinge at one extremity. A string iasteried to the other, pasams over a pully in a vertical line above the hinge, and is attached to a weight equal to half that of the rod, which rests on a curve. The length of the atring and the height of the pully above the hinge are each equal wo the length of the rod, and the system is in equilitirium is all positions. Shew that the oquation to the curve is

$$
r=\tan \sin ^{\prime} 10 \text {, }
$$

the pully being the origin and the prime radins being rertical.

3. Two rods each of length $2 a$ have their ends united at an angle $a$, and are placed in a vertical plane on a sphere of radius $r$. Prove that the equilibrium is stable or unstable according as

$$
\sin a \text { is }>\text { or }<\frac{2 r}{a} \text {. }
$$

4. A prolate spheroid rests with its amaller end on a bonzontal table. Is the equilibrium stable or unstable?

5. A cylinder rests with the centre of its base in contact with the highest point of a fixed sphere, and four times the altitude of the cylinder is equal to a great circle of the sphere; supposing the surfaces in contact to be rough enough to prevent sliding, shew that the cylinder may be made to rock through an angle of $90^{\circ}$, but not more, without falling off the spliere.

6. A very small bar of matter is moveable about one extremity which is fixed halfway between two centres of force attracting inversely as the square of the distance : if $l$ be the length of the bar, and $2 a$ the dintance between the 
centres of force, prove that there will be two positions of equilibrium for the bar, or four, according as the ratio of the absolute intensity of the more powerful force to that of the less powerful, is, or is not, greater than $\frac{a+2 l}{a-2 l}$; and distinguish between the stable and unstable positions.

7. Two particles connected by a string support each other on the arc of a vertical circle; shew that the centre of gravity is in the vertical through the centre of the circle. What is the nature of the equilibrium?

8. A sphere of radius $a$, loaded so that the centre of gravity may be at a given distance $b$ from the centre of figure, is placed on a rough plane inclined at an angle $\alpha$ to the horizon. Shew that there will be two positions of equilibrium, one stable and the other unstable, in which the distances of the point of contact from the centre of gravity are respectively,

and

$$
a \cos \alpha-\sqrt{ }\left(b^{2}-a^{2} \sin ^{2} \alpha\right),
$$

$$
a \cos \alpha+\sqrt{ }\left(b^{2}-a^{2} \sin ^{2} \alpha\right) \text {. }
$$

Hence, find the greatest inclination of the plane which will allow the sphere to rest. Is the equilibrium stable or unstable in this limiting case?

9. A sphere of radius $r$ rests on a concave sphere of radius $R$; if the sphere be loaded so that the height of its centre of gravity from the point of contact be $\frac{3}{2} r$, find $R$ so that the equilibrium may be neutral.

Result. $R=3 r$.

10. A heary cone rests with the centre of its base on the vertex of a fixed paraboloid of revolution; shew that the equilibrium will be neutral if the height of the cone be eqnal to twice the latus rectum of the generating parabola. Shew that the equilibrium is really stable.

11. A heavy particle attached to one extremity of an elastic string is placed upon a smooth curve, the string lying upon the 
curve and its other extremity being fixed to a pains in the curve; find the curve when the particle reats in all powitions. Nesult. A cycloid.

12. A uniform square board is capable of motion in a vertical plane about a hinge at one of its angular wints: a string attached to one of the nearest angular pwints, and passing over a pully pertically above the linge at a diatance from it equal to the side of the kquare, supports a weight whose ratio to the weight of the board in 1 to $\sqrt{2}$. Find the positions of equilibrium and determine whether they are respectively stable or unstable.

13. Two small smooth rings of equal weight slite on a fixed elliptical wire of which the major axis is vertical, and are connected by a string passing over a smooth preg at the upper focus; prove that the rings will rest in whatever position they may be placed.

14. A small heavy ring slides on a smooth wire in the form of a curre whose plane is rertical, and is connected by a string passing over a fixed pully in the plane of the curve with another weight which hangs freely; find the form of the curve that the ring may be in equilibrium in any position.

Result. A conic section having its focus at the pully.

15. If an elliptic board be placed, so that its plane is vertical, on two pegs which are in the same horizontal plane, there will be equilitorium if these pegs he at the extremitien of a pair of conjugate diameters. What are the limins which the distance between the pregs muat nut exised of fall shourt of, in order that this positim of equilitirium may be possible? Shew that the equilibrium is unatulite.

16. A solid of revolution, whose centre of eravity onincilen with the centre of curvature at the vertex, rests on a mough horizontal plane. Shew that the equilibrium is stable or unstable according as the value of $3\left(\frac{d^{2} y^{2}}{d x^{2}}\right)^{2}-\frac{d^{2} y}{d x^{2}}$, when $x$ and $y$ ranish, is positive or negative, $x$ and $y$ being co-ordinates of 
the gencrating curve, measured along the tangent and normal at the vertex.

17. If a plane pass through one extremity $A$ of the base of a cylinder and be inclined at an angle of $45^{\circ}$ to the axis, the piece 80 cut off will rest in neutral equilibrium, if placed with its circular end on the vertex of a paraboloid whose latus rectum is five-eighths of the diameter of the base, the point of contact being also at this same distance from $A$.

18. A piece of string is fastened at its extremities to two fixed points; determine from mechanical considerations the form which must be assumed by the string in order that the surface generated by its revolution about the straight line joining the fixed points may be the greatest possible.

\section{MISCELLANEOUS EXAMPLES.}

1. A uniform wire is bent into the form of three sides $A B, B C, C D$ of an equilateral polygon; and its centre of gravity is at the intersection of $A C$ and $B D$. Shew that the polygon must be a regular hexagon.

2. Three forces act along three straight lines which may be considered as generating lines in the same system of a hyperboloid of one sheet; shew that if the forces admit of a single resultant, it must act along another generating line of the same syatem.

3. A gate moves freely about a vertical axis, along which it also slides; while a point in the plane of the gate, and rigidly connected with it, rests on a given rough inclined plane; find the limiting position of equilibrium.

4. Suppose straight lines to be drawn from one of the centres of the four circles that touch the sides or the sides produced of a given triangle to the other three centres, and let these straight lines represent three forces in magnitude and direction; then the straight line joining the first centre with the centre of the circle circumscribing the triangle will represent in magnitude and direction one-fourth of the resultant. 
5. A particle rests in equilibrium in a fine gmove in the form of a helix, the axis of which is inclinel to the hinizon at a given angle $a$. Find the distance of the partiele from a rertical plane passing through the axis. Als find the greatest vulue of a for a given helix in order that there may be a position of equilibrium of the particle.

6. A quadrilateral figure possesges the following property: any point being taken and four trimgles formed by joining this point with the angular points of the figure, the centres of gravity of these triangles lie in the circumference of a circle: prove that the diagonals of the guadrilateral are at right angles to each other.

7. A square bonrd is supported in a horizontal pomition by three vertical strings; if one of them be atfactued to a corner, where must the others be attached in order tiat the weight which can be placed on any part of the board without overturning it may be the greatest possible?

8. A triangular plate hangs by three parallel threads attached at the comers, and supports a heary particle. Prove that if the threads are of equal strength, a heavier partiele may bo supported at the centre of gravity than at ang other point of the disc.

9. $A B C$ is a triangle; $D, E, F$ are the middle points of the sides opposite to $A, B, C$ respectively: $P$ is any point; $P D, P E, P F$ are divided in a given ratio at $A, B, O^{\prime}$ respectively: shew by the theory of the centre of gravity that A.A', $B B^{\prime}$, and $C C^{\prime}$ meet at a point.

10. A right cone is cut obliquely and then placed with its section on a horizontal plane; prove that when the angle of the cone is less than $\sin ^{-3} t$. there will be two sections for which the equilibrium is neutral, and for intermediate sections the cone will fall over.

11. A right cylinder on an elliptic base the semiaxes of which are $a$ and b) rests with its axis horizontal between two smooth inclined planes inclined at right angles to each other; 
determine the positions of equilibrium, (1) when the inclination of one of the planes is greater than $\tan ^{-1} \frac{a}{b}$, (2) when the inclination of both planes is less than $\tan ^{-1} \frac{a}{b}$.

12. A pack of cards is laid on a table; each projects in the direction of the length of the pack beyond the one below it ; if each projects as far as possible, prove that the distances between the extremities of the successive cards will form an harmonic progression.

13. Find the least excentricity of an ellipse in order that it may be capable of resting in equilibrium on a perfectly rough inclined plane.

$$
\text { Result. } e^{2}=\frac{2 \sin \alpha}{1+\sin \alpha} .
$$

14. Two mutually repelling particles are placed in a parabolic groove, and connected by a thread which passes through a small ring at the focus; shew that if the particles be at rest, either their abscissa are equal, or the two parts of the thread form one straight line.

15. Each element of a parabolic are bounded by the vertex and the latus rectum is acted on by a force in the normal proportional to the distance of the element from the axis of the parabola. Shew that the equation to the straight line in which the resultant acts is

$$
15 y+10 x=26 a \text {. }
$$

16. Each element of the are of an elliptic quadrant is acted on by a force in the normal proportional to the ordinate of that point. Shew that the equation to the straight line in which the resultant acts is

$$
6 b y-3 \pi a x+4 a^{2}-4 b^{2}=0 .
$$

17. $\mathrm{A}$ smooth body in the form of a sphere is divided into hemispheres and placed with the plane of division vertical upon a smooth horizontal plane; a string loaded at its extremities with two equal weights hangs upon the sphere, passing over its highest point and cutting the plane of division 
at richt angles; find the least weight which will preserve the equilibrium.

18. The loens of the ecntre of erarity of egmenes of equal area $A$ in an ellipse is a similar concentric ellipme whome minor axis is

$$
\frac{4}{3} \frac{a b^{2}}{A} \sin ^{2} \phi, \text { where } A=\frac{a b}{2}(\phi-\sin \phi) \text {. }
$$

19. The foci of a rough prolate spheroid attract dimectly as the distance; if a particle withous weight he placed on the spheroid, find within what limits it must he placed so as to be in equilibrium. Shew that if the coeflicient of friction be greater than $\frac{e^{2}}{2 \sqrt{\left(1-e^{3}\right)}}$, where $e$ is the excentricity, the particle will rest anywhere on the surface.

20. A circular disc of mass $m^{\prime}$ and radius e mots in contact with two equal uniform straight rods $A B, A C$, which am joined at $A$ by a smooth hinge, and which attract each other and the disc with a force varying as the distanco; also the dise attracts the rods similarly. Shew that there is equilibrium if

$$
m^{\prime} c(2 c \cos \alpha-a \sin \alpha)=m a^{2} \sin ^{\circ} \alpha \cos \alpha,
$$

where $m$ is the mass of each rod, $a$ the length of cach rod, and $2 \alpha$ their inclination to each other.

21. A square picture hangs in a vertical plane by a string. which passing over a smooth nail has its ends fastened to two points symmetrically situated in one side of the frame. Determine the positions of equilibrium, and whether they are stable or unstable.

Results. Let $l$ be the length of the string, $e$ the distance of the two points to which the ends of the string are finstened, $h$ the length of a side of the square; then if th be greater than $c \sqrt{ }\left(c^{2}+h^{2}\right)$ there is only one position of equilitrium, namely, the ordinary position, and the equilitirium is atulle: if th be less than $c \sqrt{ }\left(c^{3}+k^{3}\right)$ there are two ollique positimns of stable equilibrium, besides the ordinary position of equilibrium, which is stable with respect to some displacenents and unstable with respect to other displacements. 
22. A flexible thread is placed in a tube of any form and is acted on by any forces. The diameter of the tube is equal to that of the thread and is infinitesimal. Determine the position of equilibrium.

23. Two equal particles are connected by two given strings without weight, which are placed like a necklace on a smooth cone with its axis vertical and vertex upwards; find the tensions of the strings.

24. A triangle of area $A$ revolves through an angle $\phi$ about an axis in its own plane taken parallel to one side; shew that the least amount of surface generated is

$$
\text { A. } \phi \cdot \frac{(a+b+c)^{2}-2 a^{2}}{2(b+c) a}
$$

where $a$ is the greatest side. 


\section{EDUCATIONAL \\ MATHEMATICAL WORKS}

\section{BX \\ I. TODHUNTER, M.A., F.R.S.}

I. Euclid for Colleges and Schools. . Second Blition. $88 \mathrm{mos}$, bound in cloch. 3e. Gel.

2. Algebra for Beginners. With numerous Ex. ampleas. Now Edition. 18mo, bound in cloth. 20.64.

3. Algebra for the use of Colleges and Schools. Fourth Elition. Crown 8ro, eloth. ;0.68.

4. A Treatise on the Theory of Equations. Crown 8vo, eloth. 7o. 68.

5. Plane Trigonometry for Colleges and Schools. Third Edition, Chown 8ro, cloth. se.

6. A Treatise on Spherical Trigonometry for the

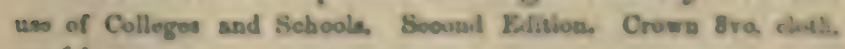
4. 6 d.

7. A Treatise on Conic Sections. With numerous

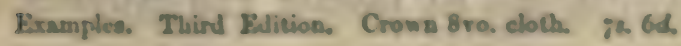


8. A Treatise on the Differential Calculus. With numerous Examplus. Fourth Edition. Crown 8vo. cloth. 308. 6 d.

9. A Treatise on the Integral Calculus. Second Edition. With numerous Examples. Crown 8vo. cloth. 10s. 6d.

x. Examples of Analytical Geometry of Three Dimeneions. Second Edition. Crown 8vo, cloth. 4 s.

Ix. A Treatise on Analytical Statics. With numerous Examples, Third Edition. Crown 8vo, eloth. 100. 6d.

12. A History of the Progress of the Calculus of Variations during the Nineteenth Century. 8vo. cloth. 128.

13. A History of the Mathematical Theory of Probability, from the time of Pascal to that of Laplace. 8vo. cloth. 18.

Edited by MR TODHUNFR.

An Elementary Treatise on Differential Equations. By GEORGE BOOLE, D.C.L., F.R.S. A New Edition, edited by I. TODhester, M.A., F.R.S. Crown 8 vo. cluth. 14 s.

A Supplementary Volume. Crown 8vo. cloth. $8 s .6 d$. 


\section{COLLEGE CLASS-BOOKS.}

\section{A TIEITISE ON TIK CAICUIVS OF FINITE UIFFRR.}

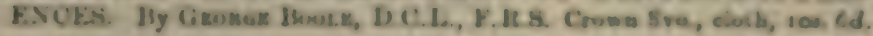

TATT ANI STEKL.K A TIFATISE ON THE DYNAMICS

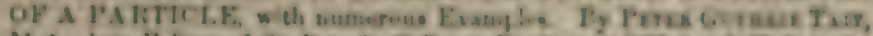

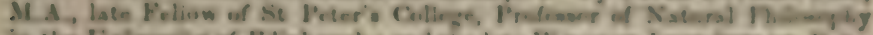

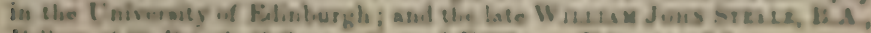

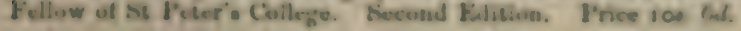

THE RLEMENTS OF PLANE AND STHEIICAL THEO.

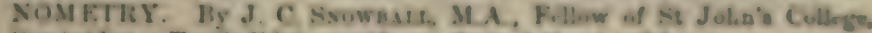

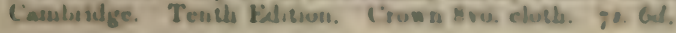

AY ELFMENTARY TREATISE ON PLANE TRIGONO.

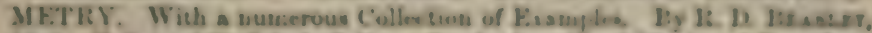

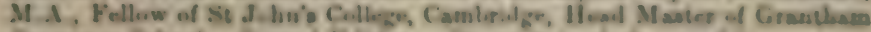

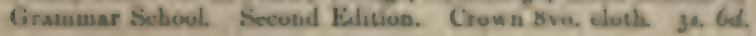

A TREATISE ON EI.EMENTAIY MECHANICX For the

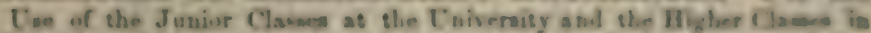

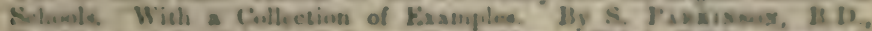
Freidens of St Jehis's College, Cambridge. Third Eitiven, reoroed. Crown 8vo, eloch. ga. Od.

A TIRATISE ON OPTIOS. By S. Pamzisisos, B.D. Cruma 8vo, eloch, 20 . $6 \mathrm{~d}$.

ELEMENTARY IYDROSTATICS. By J. B. Purak, MA.

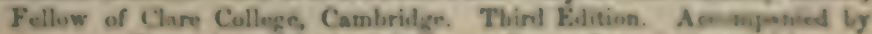
sumerous Frasuples, with the Solutiven. Crowa 8vo, cloths go fal.

A GEOMETRICAI. TREATISE ON CONIC SEOTIOSS With Coprinu Examplea frem the Cambidige Senate- Heuse Rapens. By

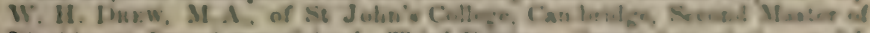

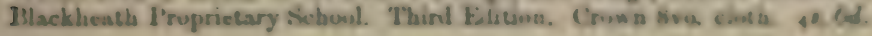

SOLUTIONS TU PROBI.EMS CONTAINED IN MR

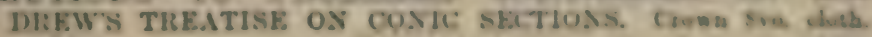
ge. Ged.

GEOMFTRICAL CONICS, incluling Anharmonie Rasio asd

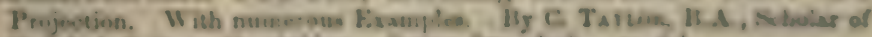

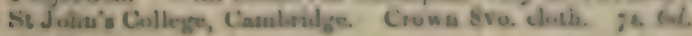

AS RI.FIENTAMY THEATISE UN (OONIC SFOTIONS

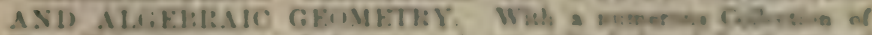

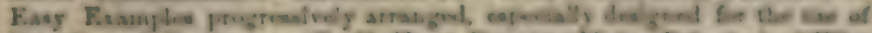

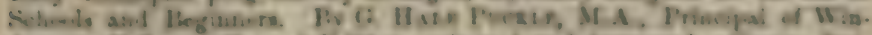

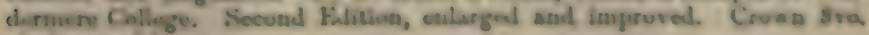
cincth. io. coll.

MACMLLAN AND CU. LONDON. 


\section{ELEMENTARY SCHOOL CLASS-BOOKS.}

The Volumes of this Series of Elemextart Scnoor Crass-Boors are hand. somely printed in a form that, it is hoped, weill assiat the young student as much as clearness of type and distinctness of arrangement can effect. They are publislied at a modernte price to ensure an extensive sale in the Schools of the linitod Kingdom and the Colonies.

\section{THE SCHOOL CIASS-BOOK OF ARITHMETIC.}

Jiv BARNARI BMITII, M.A., Lave Yellow of Bt Poter's Collego, Combridge. 18mo. limp cluth Parts I. and II. 1ud. each. Part 111. 1s.

The Three Parts complete in One Volume. 18mo. cloth. Price 8 .

KEY TO THE SCHOOL CLASS-BOOK OF ARITHMETIC. $18 \mathrm{mo}$, cloth. 6s. 6d. Or in Threo Parts, each $2.6 d$.

\section{AN ELEMENTARY IATIN GRAMMAR.}

By H. J. ROBY, M.A., I'nder Master of Dulwich Colleye Uppor Bchool, late Fellow aud Classical Lecturer of St John's Cull., Cambridgo. 18mo. 2a. Gi.

\section{MYTHOLOGX FOR LATIN VERSIFICATION.}

A Brief Sketch of the Fahles of the Ancients, prepared to be rendered into Iatin Ferse for Bchools BY P. II (I)DGSON, B.1), late Provont of Kton College. Nen Kiditiun. Heviseril by F. C. HODGsoN, M.A. Fellow of King's College, Cambridige. 18mo, 8.

\section{A LATIN GRADUAL FOR BEGINNERS.}

A First Latin Construing Book. By EDWARD THRING, M.A., Hend Marter of Upplaglam Echool. 18mo. 2s. 6d.

\section{SHAK ESPEARE'S TEMPEST.}

The Text taken from "The Cambridge Bluakespeare," With Glosanial and Explanatory Notea By the Rev. J. M. JEPHSUN, 18mu. cluth, 8. 6d.

\section{LESSONS IN ELEMENTARY BOTANY.}

The Part on Bystematic Botany based upon Material lef in Manuseript by the late Pmfensor JIEX >LOW. Wrth nearly Two Ifundred Illustrations. By JASTEL OLIVER, FR.s. F.1.S. Keejer of the llerbarium and Library of the Royal Gardens, Kew, and Profesen ir of Botany in Ciniversity College, Irundon. 18mo. cluth. 4s. 6d.

\section{AN ELEMENTARY HISTORY OF THE BOOK OF COM-} MOX PRA YER. By FRANCIS PROCTER, M.A., Viear of Witcun, Norfolk, late belluw of $8 t$ Catharine's College, Cambridge. 18mo. 2s. $6 d$.

\section{THE BIBLE IN THE CHURCH.}

A Popular Account of the Collection and Reception of the IIoly Beriptures in the Chritian Churches. By BROOKE FOSS WESTCOTT, H. A. Becond Kdition. 18mo. Is. ods

THE BIBLE WORD-BOOK.

A Glosunry of Old Engliah Bible Words. By J. RA8TWOOD, M.A., of Rt John's College, and W. ALDIS WRIGIT, M.A. Trinity College, Cambridge. 1smo. clots. So. $6 d$.

A BRIEF BIOGRAPHICAT, DICTIONARY.

Secund Edation. 18mo. cloth. 46. Gd. By bhe Rer. CHARLES HOLB, B.A.

\section{LESSONS IN ELEMENTARY PHYSIOLOGY.}

With numerous Illustrations. By T. H. H UXI,EY, F.R.S., Profeseor of Natural Hintory in the Guvernuent schuol of Mines. Nearly Beady. 16 suo. 




\section{PLEASE DO NOT REMOVE CARDS OR SLIPS FROM THIS POCKET}

\section{UNIVERSITY OF TORONTO LIBRARY}

S\&M

A

118 
